\title{
Association of killer immunoglobulin-like receptor genes with viral loads in experimental SIV infection of rhesus macaques (Macaca mulatta)
}

\author{
Dissertation \\ for the award of the degree \\ "Doctor rerum naturalium" \\ of the Georg-August-Universität Göttingen
}

submitted by

Christina Albrecht

from Göttingen

Göttingen 2012 


\section{Professor Dr. Lutz Walter}

Primate Genetics Laboratory, German Primate Centre

\section{Professor Dr. Holger Reichardt}

Department of Cellular and Molecular Immunology, Medical School Göttingen, Georg-August-University

\section{Professor Dr. Michael Kessel}

Department of Developmental Biology, Max Planck Institute for Biophysical Chemistry

Date of the oral examination: 17.09.2012 


\section{$\underline{\text { Declaration }}$}

I hereby declare that this submission is my own work and that, to the best of my knowledge and belief, it contains no materials previously published or written by another person nor material which to a substantial extent has been accepted for the award of any other degree of the university or other institute of higher education, except where due acknowledgment has been made in the text.

Christina Albrecht

Signature
Name
Göttingen, 07.08.2012

Date and place 


\section{Table of contents}

List of figures ................................................................................................

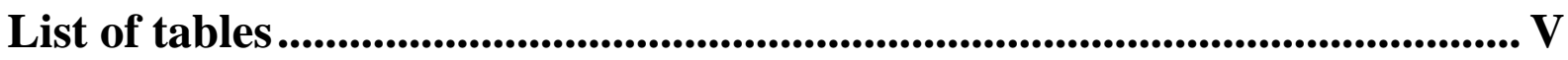

List of abbreviation ....................................................................................................

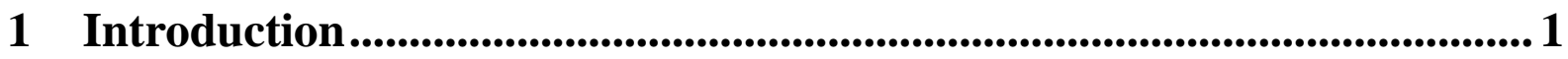

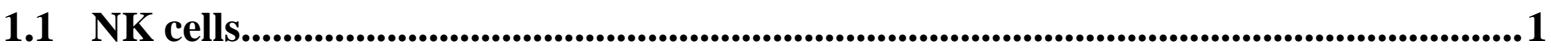

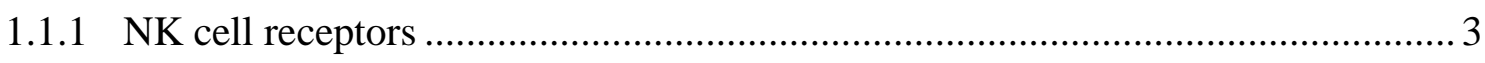

1.2 Killer immunoglobulin-like receptors .............................................................................4

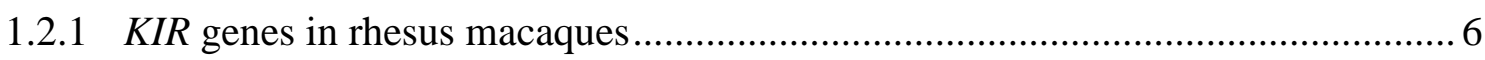

1.3 Major histocompatibility complex $(\mathrm{MHC})$ class I genes....................................................8

1.3.1 MHC class I genes in rhesus macaques ........................................................ 8

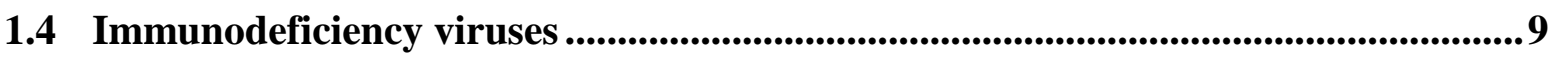

1.4.1 NK cells and KIRs in HIV/SIV infection ................................................. 12

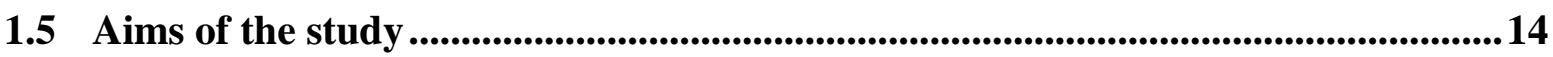

2 Material and Methods $\ldots \ldots \ldots \ldots \ldots \ldots \ldots \ldots \ldots \ldots \ldots \ldots \ldots \ldots \ldots \ldots \ldots \ldots \ldots \ldots \ldots \ldots \ldots \ldots \ldots \ldots \ldots \ldots \ldots . \ldots \ldots$

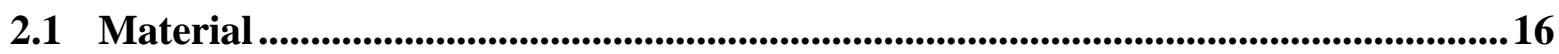

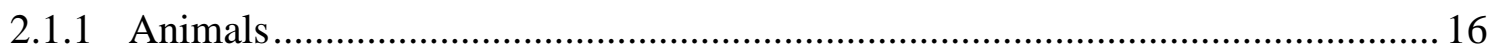

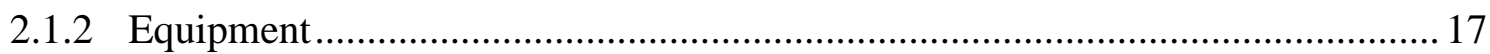

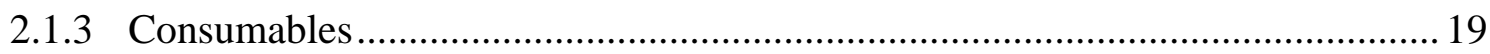

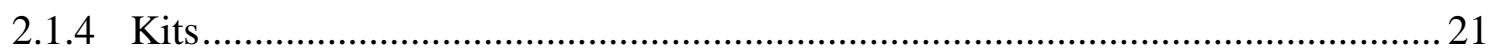

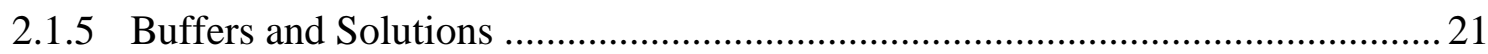

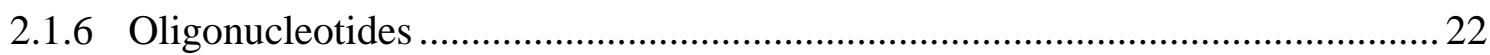

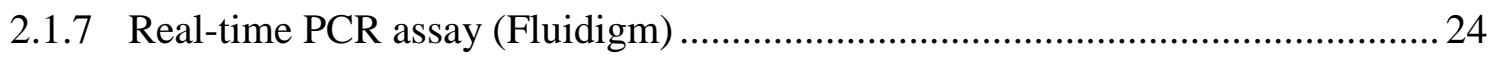

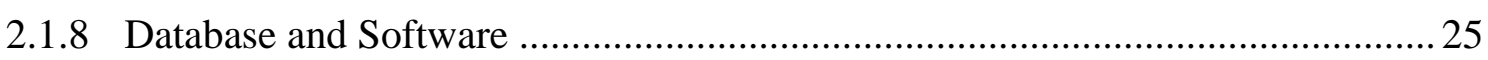

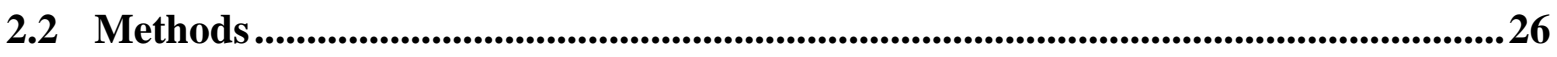

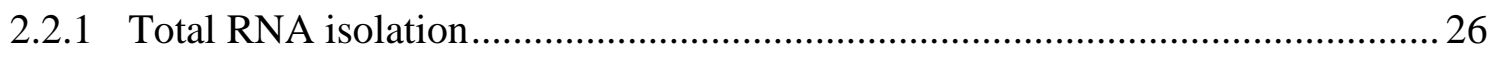

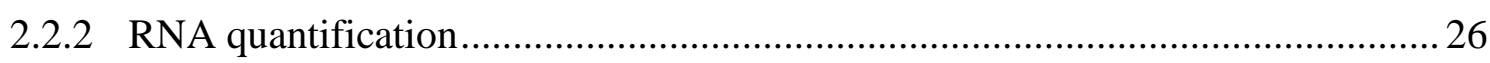

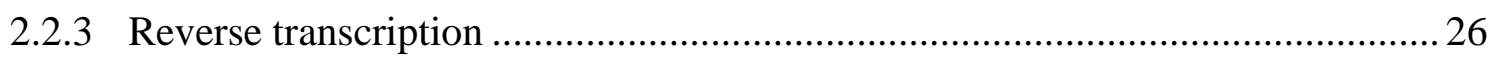

2.2.4 Amplicon preparation by Polymerase Chain Reaction (PCR) ........................... 26

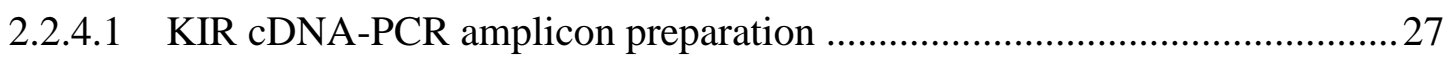

2.2.4.2 MHC class I cDNA-PCR amplicon preparation ........................................28

2.2.5 Gel electrophoresis of DNA in agarose gels...................................................... 28 


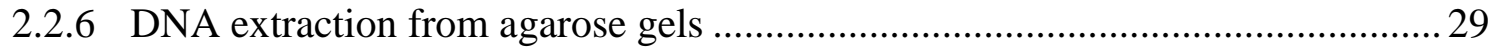

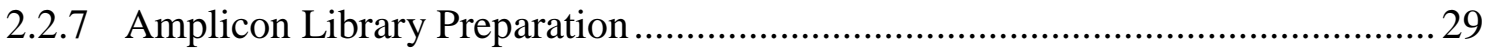

2.2.8 454 Sequencing (GS Junior System, Roche) .................................................... 29

2.2.9 Bioinformatic analysis of 454 Sequencing data (KIR) ..................................... 30

2.2.10 Bioinformatic analysis of 454 Sequencing data (MHC class I) ......................... 31

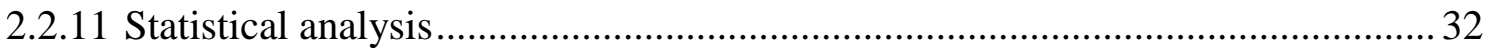

2.2.12 Quantitative real-time PCR gene expression with the Biomark ${ }^{\mathrm{TM}}$ System

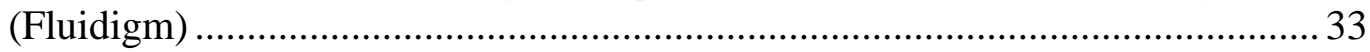

2.2.13 Determination of relative gene expression ...................................................... 34

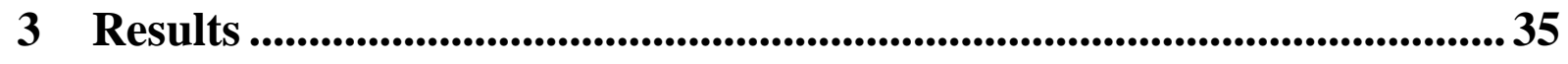

3.1 Best fitting approach to analyse 454 sequencing data for $K I R$ transcripts.............37

3.2 Characterisation of KIR and MHC class $I$ genes of the rhesus macaque cohort ... 42

3.2.1 Frequency of distinct KIR transcripts in the rhesus macaque cohort.................... 42

3.2.2 Interindividual variation in expression levels of $K I R$ transcripts ....................... 44

3.2.3 Investigation of interindividual differences of KIR transcripts ......................... 44

3.2.4 Diversity of $M H C$ class $I$ transcripts in the rhesus macaque cohort.................... 46

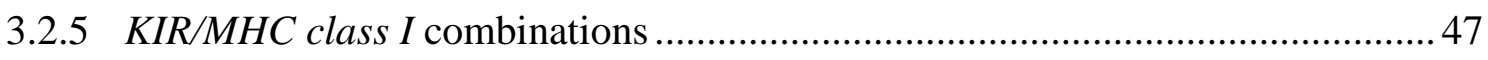

3.3 Investigation of $K I R$ and $M H C$ class $I$ transcripts and viral loads .......................49

3.3.1 Comparison of the frequencies of transcribed KIR genes according to plasma

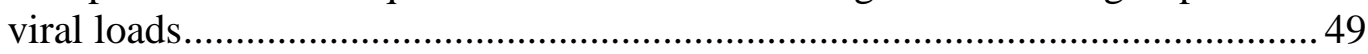

3.3.2 Relative expression levels of KIR transcripts in the HVL and LVL cohort (pre infection) ....................................................................................... 51

3.3.3 Comparison of KIR3DL08 transcription between the HVL and LVL cohort...... 53

3.3.4 Comparison of $M H C$ class I transcript profiles according to plasma viral

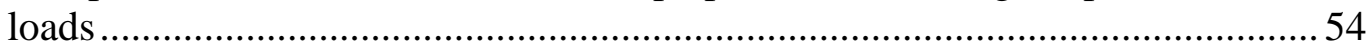

3.4 Changes in KIR transcription upon SIV infection ......................................................55

3.4.1 SIV-dependent changes of the relative expression levels of KIR transcripts ...... 56

3.4.2 SIV-dependent changes of the relative KIR transcription are associated with

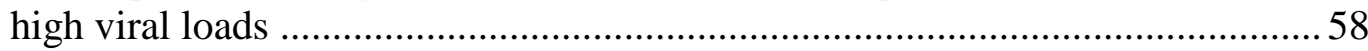

3.4.3 Inverse correlation of KIR3DL05 and KIR3DL07 transcription levels ............... 61

3.4.4 Investigation of interindividual changes of KIR transcription levels over the

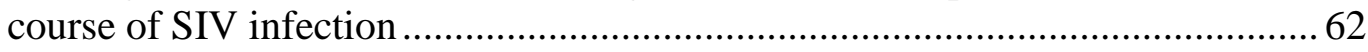

3.5 Comparison of the KIR transcript repertoire of $\mathrm{NK}$ cells and $\mathrm{CD8}^{+} \mathrm{T}$ cells ......... 65

3.6 Gene expression analysis of SIV-infected rhesus macaques by real-time PCR .... 66

4 Discussion................................................................................. 72

4.1 Diversity of rhesus macaque $K I R$ and $M H C$ class $I$ transcripts............................73 
4.1.1 Diversity of rhesus macaque KIR gene transcription ................................... 73

4.1.2 Diversity of rhesus macaque $M H C$ class I transcription ................................... 74

4.2 Associations of KIR transcription and viral replication .....................................75

4.2.1 KIR3DL transcripts are associated with viral replication .................................. 76

4.2.2 Higher KIR3DL05 and KIR3DL08 transcription levels are associated with viral replication and SIV disease progression.............................................. 77

4.3 Phenotypic changes of immune cell marker upon experimental SIV infection .... 79

4.4 Models to explain advantageous and disadvantageous KIR in SIV infection .......81

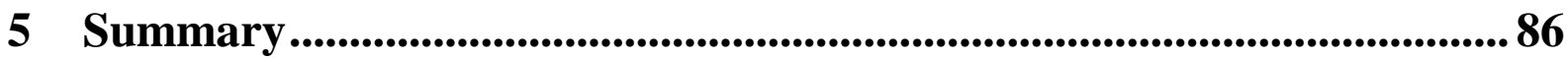

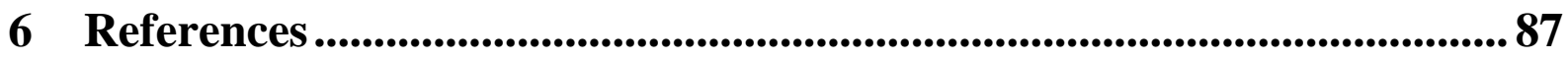

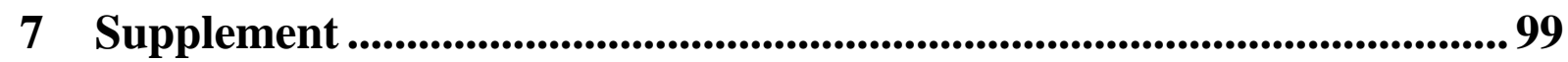

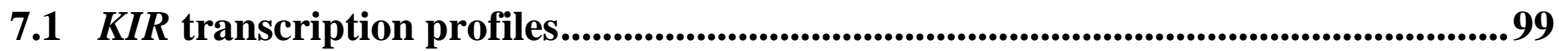

7.2 MHC class I transcription profiles.........................................................................101

7.3 Gene expression analyses (fold changes post infection with SIV)..........................106

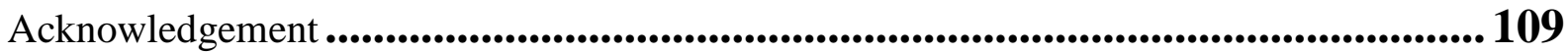




\section{List of figures}

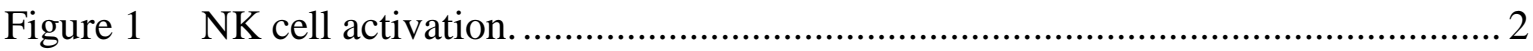

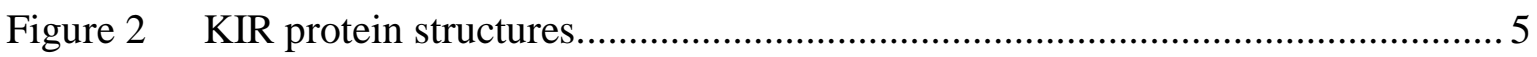

Figure 3 Schematic representation of rhesus macaque KIR3D and KIR2D genes........... 7

Figure 4 Schematic comparison of the MHC class I region in humans and rhesus

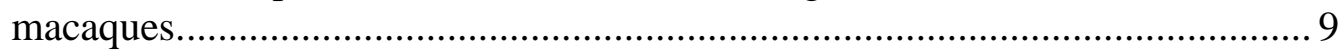

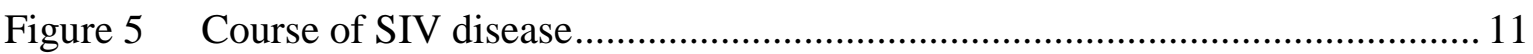

Figure 6 Nucleotide sequences of the 454 GS Junior amplicon primers. ....................... 27

Figure 7 Experimental set-up for comprehensive KIR typing using 454 sequencing..... 37

Figure 8 Relative expression levels of KIR transcripts in the macaque cohort

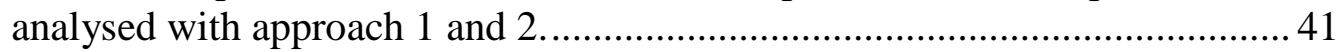

Figure 9 Identified KIR transcripts in the rhesus macaque cohort (pre infection).......... 43

Figure 10 Relative contribution of $K I R$ transcripts in the rhesus macaque cohort

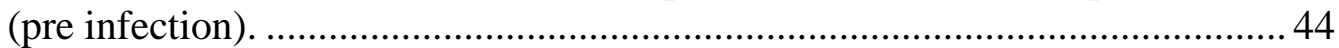

Figure 11 Interindividual differences of three different $K I R$ transcription profiles.......... 46

Figure 12 Frequencies of KIR transcripts in the HVL and LVL cohort (pre

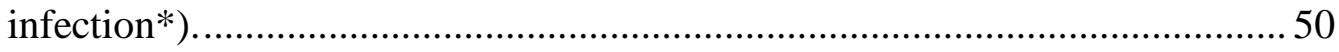

Figure 13 Comparison of the relative transcription levels of KIRs in the HVL and LVL cohort (pre infection).

Figure 14 Comparison of KIR3DL05 transcription levels between the HVL and LVL

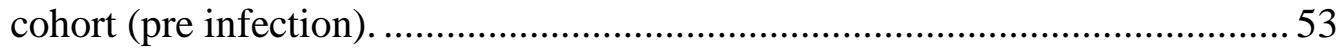

Figure 15 Comparison of KIR3DLO8 transcription in $K_{I R 3 D L O 8^{+}}$individuals (pre infection).

Figure 16 Changes of KIR3DL05 and KIR3DL07 transcription levels in the course of SIV infection

Figure 17 Changes of KIR3DL05 and KIR3DL07 transcription levels in the HVL and LVL cohort over the course of SIV infection.....

Figure 18 SIV-dependent changes of KIR transcription levels in the HVL and LVL cohort.

Figure 19 Correlation analysis of KIR3DL05 and KIR3DL07 (KIR3DL01) transcription levels.

Figure 20 Comparison of constant KIR transcription profiles over course of SIV infection.

Figure 21 Comparison of three KIR transcription profiles over the course of SIV infection.

Figure 22 Comparison of KIR transcription profiles of NK cells and CD8 ${ }^{+} \mathrm{T}$ cells. ....... 66

Figure 23 Gene expression analysis of NK cell markers in the HVL and LVL cohort post infection with SIV.

Figure 24 Gene expression analysis of activation marker in the HVL and LVL cohort post infection with SIV

Figure 25 Gene expression analysis of T- and B-cell markers in the HVL and LVL cohort post infection with SIV

Figure 26 Advantageous and disadvantageous expression of inhibitory KIRs in SIV disease. 


\section{List of tables}

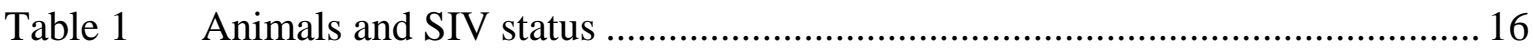

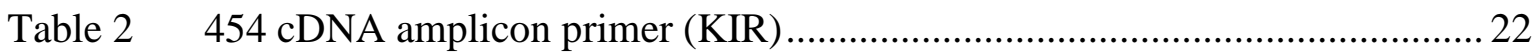

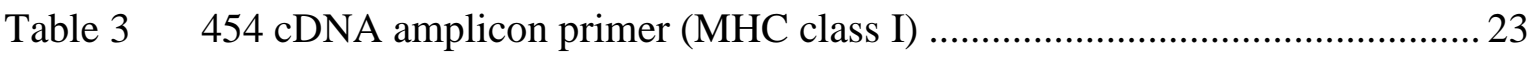

Table 4 DELTAgene Assay for qPCR (gene expression) .......................................... 24

Table 5 Comparison of the relative expression levels of KIRs of individual mm5 established with approach 1 and 2 ............................................................ 39

Table 6 Comparison of two different modifications of approach 2 (individual

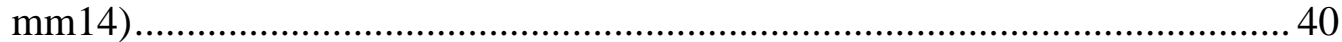

Table 7 Frequencies of transcribed KIRs in the rhesus macaque cohort (pre

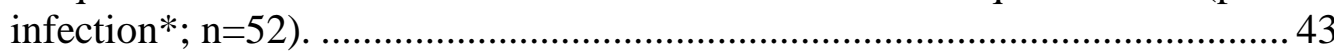

Table 8 Frequencies of transcribed KIR genes in the HVL and LVL cohort (pre

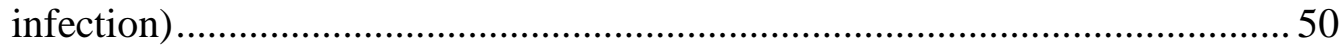

Table 9 Differences of the relative expression levels of KIRs between the HVL and LVL cohort (pre infection) ......................................................................... 52 


\section{List of abbreviation}

${ }^{\circ} \mathrm{C}$
$\mu \mathrm{l}$

AIDS

bp

CCR

CD

cDNA

DC

DNA

dNTP

DPZ

e.g.

EC

EDTA

emPCR

et al.

$\mathrm{Fc}$

fwd

g

GAPDH

GZMB

$\mathrm{h}$

HIV

HLA

HPLC

HPRT1

HVL

IFN

Ig

IL

ITAM

ITIM

KIR

1

LAMP1

LRC

LTNP

LVL

$\mathrm{M}$

MCMV

MHC

MID degree Celsius

microlitre

acquired immune deficiency syndrome

base pair

CC chemokine receptor

cluster of differentiation

complementary DNA

dendritic cell

deoxyribonucleic acid

deoxyribonucleotide

Deutsches Primatenzentrum

exempli gratia

elite controller

ethylenediaminetetraacetic acid

emulsion PCR

et alteri

fragment crystallisable

forward

gram

glycerinaldehyd-3-phosphat-dehydrogenase

granzyme B

hour

human immunodeficiency virus

human leukocyte antigen

high performance liquid chromatography

hypoxanthine phosphoribosyltransferase 1

high viral load

interferon

immunoglobulin

interleukin

immunoreceptor tryrosine-based activating motif

immunoreceptor tryrosine-based inhibitory motif

killer immunoglobulin-like receptor

litre

lysosomal-associated membrane protein 1

leucocyte receptor complex

long-term non-progressor

low viral load

Molar

murine cytomegalovirus

major histocompatibility complex

multiplex identifiers 


$\begin{array}{ll}\text { min } & \text { minute } \\ \text { ml } & \text { millilitre } \\ \text { mm } & \text { Macaca mulatta } \\ \text { NCR } & \text { natural cytotoxicity receptor } \\ \text { NK } & \text { Natural killer } \\ \text { NKC } & \text { natural killer complex } \\ \text { NKG } & \text { natural killer group } \\ \text { nt } & \text { nucleotide } \\ \text { PBMC } & \text { peripheral blood mononuclear cell } \\ \text { PBS } & \text { Phosphate buffered saline } \\ \text { PCR } & \text { Polymerase Chain Reaction } \\ \text { pH } & \text { potentia Hydrogenii } \\ \text { PRF-1 } & \text { perforin-1 } \\ \text { qPCR } & \text { quantitative real-time PCR } \\ \text { rev } & \text { reverse } \\ \text { RNA } & \text { ribonucleic acid } \\ \text { RNase } & \text { ribonuclease } \\ \text { SD } & \text { standard derivation } \\ \text { sec } & \text { second } \\ \text { SIV } & \text { simian immunodeficiency virus } \\ \text { SNP } & \text { single nucleotide polymorphisms } \\ \text { SSP } & \text { sequence-specific PCR } \\ \text { STA } & \text { specific target amplification } \\ \text { Taq } & \text { Thermus aquaticus } \\ \text { TBE } & \text { tris/Borate/EDTA buffer } \\ \text { TBS } & \text { tris-Buffered Saline } \\ \text { TCR } & \text { T cell receptor } \\ \text { TE } & \text { tris/EDTA buffer } \\ \text { TNF } & \text { tumour necrosis factor } \\ \text { U } & \text { unit } \\ \text { UV } & \text { ultraviolet } \\ \text { V } & \end{array}$

DNA-base abbreviations

\begin{tabular}{ll} 
Base & Nucleotide code \\
\hline Adenine & $\mathrm{A}$ \\
Cytosine & $\mathrm{C}$ \\
Guanine & $\mathrm{G}$ \\
Thymine & $\mathrm{T}$
\end{tabular}




\section{Introduction}

\subsection{NK cells}

The immune system is classically divided into innate and adaptive immunity. The innate immunity is characterised by a multiplicity of myeloid and lymphoid cells that exert rapid effector function via a limited repertoire of germline-encoded receptors. In contrast $\mathrm{T}$ and $\mathrm{B}$ lymphocytes clonally expressing a large repertoire of antigen-specific receptors, which are produced by site-specific somatic recombination, are crucial components of the adaptive immunity (Vivier et al., 2011; Medzhitov and Janeway, 1998).

Human natural killer (NK) cells are large, bone marrow-derived, granular lymphocytes, which comprise approximately $15 \%$ of the lymphocytes circulating in peripheral blood. In contrast to $\mathrm{T}$ and $\mathrm{B}$ lymphocytes, NK cells represent a unique subset of lymphocytes that do not express an antigen-specific receptor and have the ability to lyse target cells without the need for prior sensitization (Herberman et al., 1975; Kiessling et al., 1975; Cooper et al., 2001). They are essential components of the innate immune system and are important players in an effective antiviral and antitumor immune response (Biron et al., 1999; Cerwenka and Lanier, 2001). Over the past years NK cells were shown to be more complex than originally anticipated. They can reject bone marrow transplants, play important roles during pregnancy, and autoimmunity (Yokoyama et al., 2004; Moffett-King, 2002). Moreover, NK cells can directly regulate the adaptive immune response by producing pro-inflammatory cytokines or indirectly by interacting with dendritic cells (DCs) and $\mathrm{T}$ cells to shape and magnitude the adaptive immune response (Cooper et al., 2001; Fernandez et al., 1999; Mailliard et al., 2003). The two major functions of NK cells, recognition and killing of target cells on the one hand, and the production of cytokines on the other hand, are carried out by different NK cell populations, which are divided into two subsets based on their surface density of CD56 (Lanier et al., 1986). Approximately $10 \%$ of all blood NK cells express high densities of CD56 (CD56 ${ }^{\text {bright }}$ ) and represent an immature state of NK cells. CD56 $6^{\text {bright }}$ NK cells express natural cytotoxicity receptors (NCRs) and C-type lectins and have the function to secrete abundant quantities of pro-inflammatory cytokines but lack the function to kill target cells. The major population of blood NK cells express low levels of CD56 (CD56 ${ }^{\mathrm{dim}}$ ) and are thought to be the more mature phenotype. CD56 ${ }^{\mathrm{dim}} \mathrm{NK}$ cells express abundant levels of killer immunoglobulin-like receptors (KIRs) and are able to lyse virus-infected and certain tumour cells spontaneously (Cooper et al., 2001). 
The function of NK cells appears to be regulated by a complex balance system of inhibitory and activating receptor-ligand interactions (Lanier, 2005). Signals that inhibit NK cells are mainly achieved through interaction with major histocompatibility complex (MHC) class I molecules via NK cell receptors that suppress NK cell activation more than initiate NK cell function (Figure 1A). This mechanism protects healthy cells from autologous NK cell attack. MHC class I molecules are expressed on the cell surface of almost all nucleated cells and present intracellularly processed peptides to cytotoxic T cells (Karlhofer et al., 1992). In aberrant cells the expression of MHC class I molecules can be down-regulated to avoid recognition by cytotoxic T cells (Garcia-Lora et al., 2003; Orange et al., 2002). Nevertheless, the recognition by NK cells is not inhibited, as the lack of inhibitory signals by self MHC class I molecules, along with the engagement of activating receptors by activating ligands on potential target cells results in the lysis of the target cell (Figure 1B) (Moretta et al., 2001). This mechanism of immune surveillance was described by the "missing-self" hypothesis, postulating that NK cells kill target cells, like virally infected cells and tumour cells by detecting the deleted or reduced expression of self-MHC class I molecules (Kärre et al., 1986; Ljunggren and Kärre, 1990).
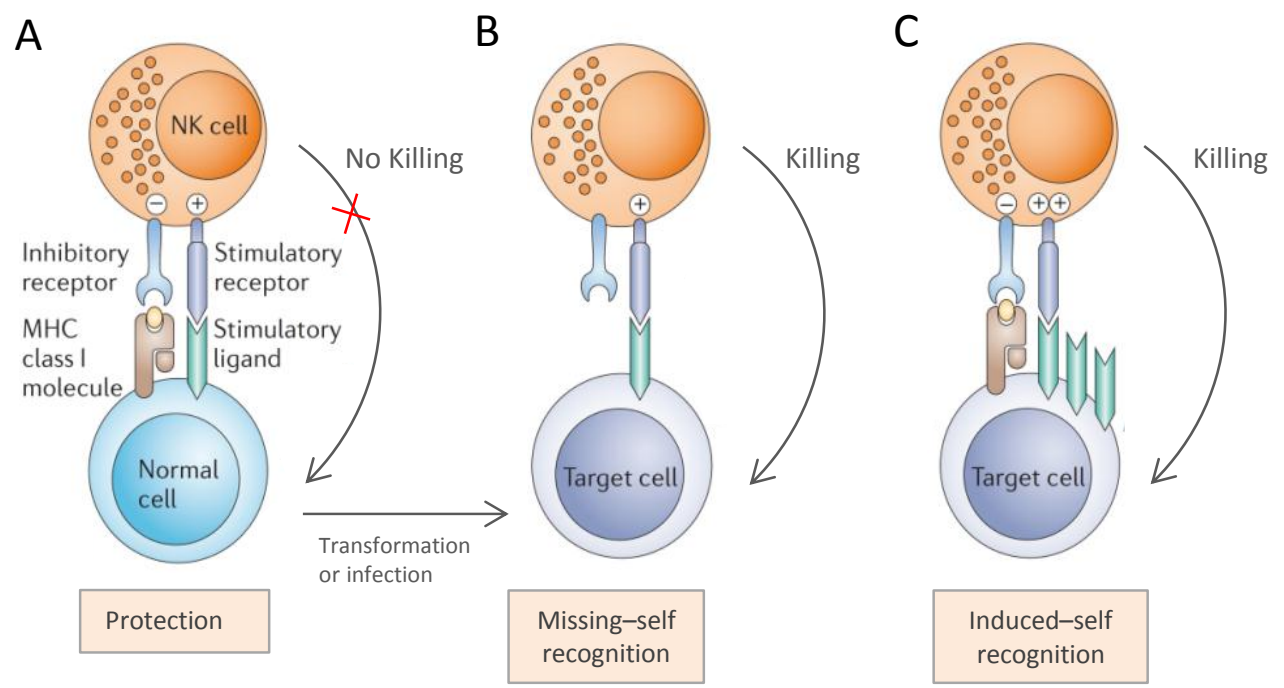

Figure 1 NK cell activation.

(A) Normal cells expressing host MHC class I alleles are protected from killing by NK cells via engagement of inhibitory KIR receptors expressed on the NK cell. Signals delivered by stimulatory receptors are balanced by inhibitory signals delivered by self MHC class I molecules. (B) Viral infections and transformation can cause down-regulation of MHC class I molecules on the surface of target cells. In this way, the NK cell does not receive inhibitory signals and therefore lyses the target cells (known as "missing-self recognition"). (C) Viral infections and transformation might also induce expression of stimulatory ligands such that constitutive inhibition delivered by inhibitory receptors is overcome by the activating signal (known as "induced-self recognition") (Figure modified from Raulet and Vance, 2006). 
Expression of endogenous self-molecules like MICA/B can be induced due to infections, tumorigenic process, or other stress. These self-molecules provide ligands for activating NK cell receptors, such that constitutive inhibition delivered by inhibitory receptors is overcome (“induced cell recognition"; Figure 1C) (Gasser et al., 2005; Raulet and Vance, 2006).

\subsubsection{NK cell receptors}

NK cells express an abundant subset of inhibitory and activating receptors, thus, modulating their function (Lanier 2005). Some receptors are unique to NK cells, such as the NCRs, including the triggering NKp30, NKp44, and NKp46 (Moretta et al., 2001; Pessino et al., 1998), while others are expressed on different other lymphocyte subsets as well. Two main families of receptors that recognise MHC class I antigens have received the most attention, the C-type lectins (NKG2) and KIRs, which are arranged in two clusters, the natural killer complex (NKC) and the leukocyte receptor complex (LRC) (Lanier 1998; Trowsdale et al., 2001; Vilches and Parham, 2002). The NKC on human chromosome 12p13.1 encodes C-type lectin molecules including CD94/NKG2- and NKG2D receptors. The C-type lectins are a family of inhibitory and activating receptors, which are conserved across mice and humans (Kelley et al., 2005). Besides their role in effector response of NK cells, they are supposed to be linked to early NK cell development (Freud and Caligiuri, 2006). The family of NK cell receptors that was intensively investigated in this study are members of the KIR family (Bashirova et al., 2006). KIRs belong to the immunoglobulin (Ig)-superfamily and are encoded within the LRC on human chromosome 19q13.4. (Wilson et al., 1997; Wende et al., 1999; Lanier, 1998). While the KIR receptors are the most important receptors that are expressed on NK cells in human and most other primates, Ly49 carries out the analogous function in mice (Natarajan et al., 2002). The human genome contains a non-functional Ly49 gene (Westgaard et al., 1998), and two KIR-like genes were found in mice (Welch et al., 2003). However, both genes are neither phylogenetically nor structurally related and have diverged between primates and rodents since their last common ancestor. 


\subsection{Killer immunoglobulin-like receptors}

KIR molecules belong to the Ig-superfamily and are expressed on the surface of NK cells and subsets of T cells (Trowsdale et al., 2001; Wagtmann et al., 1995; Uhrberg et al., 2001; D`Andrea and Lanier, 1998). They interact with MHC class I molecules on normal cells and target cells and mediate inhibitory or activating signals. Unlike the highly conserved C-type lectins, KIR genes are encoded within a region that has undergone expansions and contractions over time. Following the $M H C$, the KIR genes are located within the second most polymorphic region within the human genome. Human KIR haplotypes are diversified by their gene content and allelic polymorphism allowing for enormous variability in the number and combination of certain KIR genes in different individuals (Vilches and Parham, 2001; Uhrberg et al., 1997; Shilling et al., 2002). The diverse number of genes in KIR haplotypes occurs due to extensive gene duplications and non-reciprocal crossing-over events that are facilitated by the sequence similarity of $K I R$ introns and intergenic regions and by the proximity of the genes (Martin et al., 2000; Wende et al., 2000). So far 16 distinct KIR genes have been identified in human but the number of KIR genes identified per haplotype between individuals is variable (Wilson et al., 2000; Uhrberg et al., 2002; Hsu et al., 2002; Middleton and Gonzelez, 2010).

Two major haplotype groups have emerged, termed A and B, providing a framework for their genomic order (Uhrberg et al., 1997). The A haplotype has been identified to contain only one activating receptor (KIR2DS4), B haplotypes on the other hand are more variable and are characterised by the presence of more than one activating KIR gene (Uhrberg et al., 1997; Vilches et al., 2000). Both haplotypes share three more or less conserved framework genes, KIR2DL4, KIR3DL3, and KIR3DL2, respectively.

Three criteria have been used to classify the encoded KIR proteins, the number of extracellular Ig-like domains, the length of the cytoplasmic tail, and sequence similarity (Vilches and Parham, 2002) (Figure 2). KIR proteins can have two or three Ig-like domains and are named accordingly, KIR2D or KIR3D. The Ig-like domains mutually interact to form the binding site for MHC class I proteins (Boyington and Sun., 2002). The effector functions of KIRs are different, as they can mediate inhibitory or activating signals, which are determined by the sequence of the transmembrane region and the cytoplasmic tail (Vély and Vivier, 1997). Inhibitory receptors have long cytoplasmic tails (KIR2DL or KIR3DL) containing immunoreceptor tyrosine-based inhibitory motifs (ITIMs), which decrease activation (Long, 1999; Stebbins et al., 2003). In contrast, activating receptors have short cytoplasmic tails (KIR2DS or KIR3DS) with a positively charged residue in the 
transmembrane domain, facilitating association with the ITAM-containing adaptor molecule DAP12 (DNAX-activation protein 12) (Vivier et al., 2004). Different KIRs with a similar organization but a sequence divergence of more than $2 \%$ are numbered in series (Marsh et al., 2002).

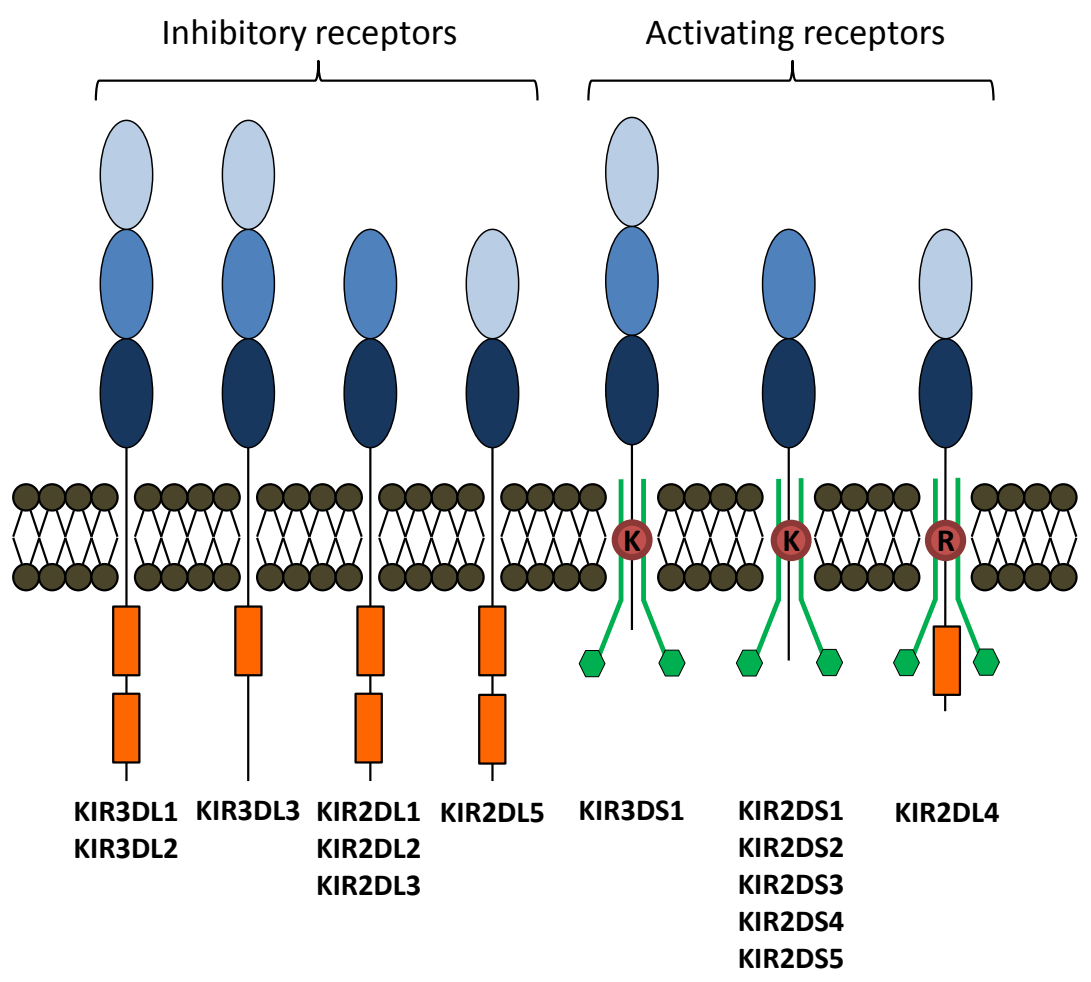

Figure 2 KIR protein structures.

The structural characteristics of two and three Ig-like domain KIR proteins are shown. The inhibitory KIR2DL and KIR3DL proteins are shown along with their long cytoplasmic tails usually containing two ITIMs (orange boxes). The short cytoplasmic tails of activating KIR2DS and KIR3DS proteins possess a positively charged amino acid residue (K=Lysin) in their transmembrane region, which allows them to associate with a DAP12 signalling molecule. KIR2DL4 is an exception, as it contains only one ITIM and a positively charged amino acid residue ( $\mathrm{R}=$ Arginin) in its transmembrane region (Figure modified from IPD KIR database).

The $K I R$ genes identified to date have been divided into four different lineages. Lineage I comprises KIR2D genes with a specific Ig D0 and D2 configuration (KIR2DL4, KIR2DL5) whereas KIR2D genes with an Ig D1 and D2 domain belong to lineage III. Genes with three Ig domains (KIR3D) belong to lineage II, except for KIR3DL3, which exclusively represents lineage V (Rajalingam et al., 2004; Guethlein et al., 2007). The majority of human KIR molecules contains two Ig domains and is able to interact with human leukocyte antigen (HLA)-C molecules. HLA-A and -B encode ligands for KIR molecules with three Ig domains (Parham et al., 2010). 
KIRs are clonally expressed such that individual NK cells within a person express apparently stochastic combinations of $K I R$ genes. This stochastic activation of $K I R$ genes, leading to distinct percentages of NK cells that express a given KIR, are mainly regulated at the transcriptional level and are stably maintained in healthy individuals (Valiante et al., 1997; Vilches and Parham, 2002). Restricted access to promoter regions as a result of the methylation state of active versus silent $K I R$ genes represents an important epigenetic mechanism of regulating KIR gene transcription. A CpG island, which is the main target of methylation, surrounding the transcriptional start site of each $K I R$ gene correlates with the expression status of KIR genes (Santourlidis et al., 2002; Chan et al., 2003). KIR genes with a methylated start site are not expressed, while demethylation leads to the expression of that $K I R$. A genetic level of transcriptional control and the variegated expression patterns of NK cells within a single donor are exerted by the promoter activity (Stulberg et al., 2007).

\subsubsection{KIR genes in rhesus macaques}

$K I R$ genes have diverged dramatically between different primate species, consistent with rapid, species-specific expansion of the gene family (Sambrook et al., 2005). In non-human primates KIR genes have been described for gorillas (Rajalingam et al., 2004), chimpanzees (Khakoo et al., 2000), bonobos (Rajalingam et al., 2001), orangutans (Guethlein et al., 2002), rhesus macaques (Hershberger et al., 2001; Sambrook et al., 2005), cynomolgus macaques (Bimber et al., 2008) and African green monkeys (Hershberger et al., 2005). Only a minority of $K I R$ genes are conserved between these species and mostly being specific to one or two species (Sambrook et al., 2005). KIR2DL4 is the only orthologous KIR gene found in human, chimpanzees, gorillas, African green monkeys, and rhesus macaques (Rajalingam et al., 2004).

Based on physiological similarities to humans, the rhesus macaque (Macaca mulatta) is one of the most important animal models to study the onset, progression, and outcome of diverse infectious diseases (Bontrop and Watkins, 2005). By now only one complete rhesus macaque $K I R$ haplotype has been published (Sambrook et al., 2005), comprising five different KIR genes, which are members of KIR lineage I, II and, V but without any activating genes. However, several recent studies pointed out that the number of KIR genes varies substantially between rhesus macaque individuals (5-11 KIR genes per haplotype) and that individuals possess at least one activating KIR receptor per haplotype (Kruse et al., 2010; Blokhuis et al., 2009; Blokhuis et al., 2011). 
KIR molecules in rhesus macaques differ from KIR molecules identified in humans. The majority of human KIR molecules contain two Ig domains (lineage I and III) and only three KIR3DL molecules (2 lineage II, 1 lineage V) are known. In contrast, considerable diversity of lineage II KIRs was detected in rhesus monkeys, indicating that extensive evolution has occurred in this receptor family (Hershberger et al., 2001). These KIR3D molecules are organised in 9 exons that correspond to the functional areas of the KIR molecule (Figure 3) (Martin et al., 2000). The activating KIR3DS genes in rhesus macaques were first designated as $\mathrm{KIR} 3 \mathrm{DH}$ ( $\mathrm{H}=$ hybrid), because they have characteristics of both KIR3DL and KIR2DL4 molecules. KIR3DL molecules exhibit sequence similarity to the transmembrane and cytoplasmic region of KIR2DL4, but a 53 nucleotide (nt) deletion results in the early termination and the loss of the ITIMs. In contrast to human, only a single lineage III KIR of unknown function (KIR1D) and a single lineage I gene (KIR2DL4) is known in rhesus macaques. In human and chimpanzee KIR2DL4 molecules contain one ITIM, while KIR2DL4 in rhesus macaques is distinguished by two ITIMs in the cytoplasmic domain (Selvakumar et al., 1997; Khakoo et al., 2000; Hershberger et al., 2001).

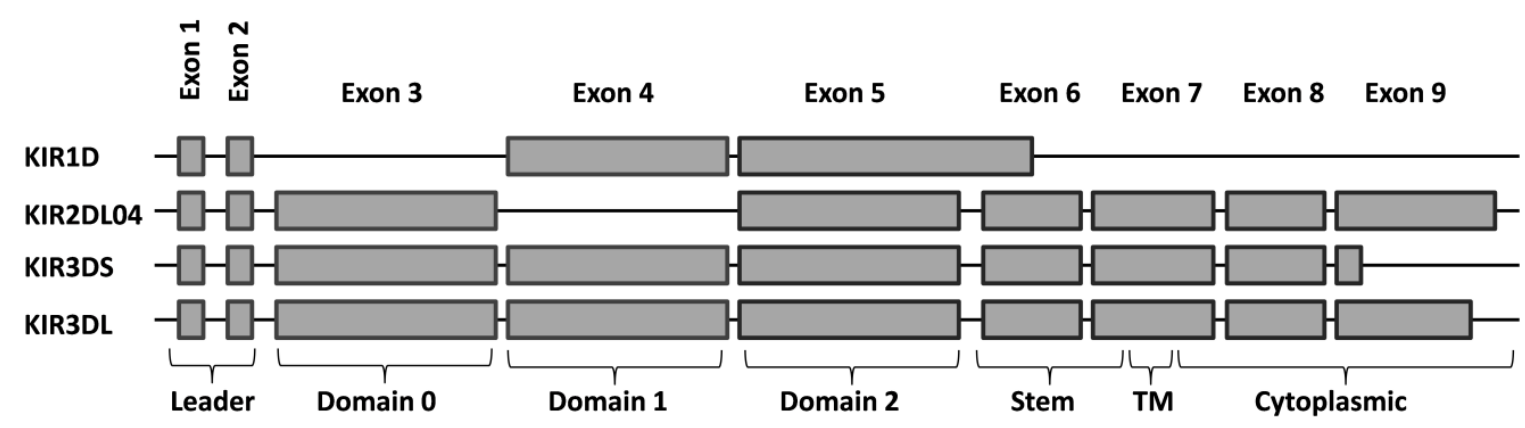

Figure 3 Schematic representation of rhesus macaque KIR3D and KIR2D genes.

The exons of each KIR gene are shown along with the domain structure. $T M=$ Transmembrane region

The expansion of lineage III KIRs in human and lineage II KIRs in rhesus macaques is a crucial difference between these two species (Guethlein et al., 2007; Sambrook et al., 2005) and likely resulted from coevolution with their cognate MHC class I ligands. In rhesus macaques the MHC class I genes that correspond to human HLA-A and $-B$ genes are considerably expanded (Otting et al., 2005, 2007), which is consistent with the expansion and diversity of lineage II KIR genes. The emergence and fixation of MHC-C ligands, which are missing in rhesus macaques, is associated with the expansion of lineage III KIR genes in humans and great apes (Parham et al., 2010). 


\subsection{Major histocompatibility complex (MHC) class I genes}

The $M H C$ is located on chromosome 6p21.3 in human, and 6q24 in rhesus macaques. It is a dense cluster of genes particularly represented by the highly polymorphic MHC class I and class II genes, which are essential parts of the immune system (Huber et al., 2003).

MHC class I molecules (in human also known as HLA class I) are expressed on the cell surface of almost all nucleated cells. They present intracellularly processed peptides to cytotoxic $\mathrm{T}$ cells and therefore are central elements of adaptive immunity. Moreover, they provide ligands for inhibitory MHC class I-specific NK cell receptors and engagement suppresses the NK cell-mediated killing of the target cell (Karlhofer et al., 1992; Yokoyama, 2002). In human six functional MHC class I genes that are either highly polymorphic (HLA-A, $-B$ and $-C$ ) or highly conserved (HLA-E, $-F$ and $-G$ ) are described (Robinson et al., 2003; Ishitani et al., 2003). All these MHC class I genes encode ligands for NK cell receptors, except for HLA-F, for which a function still needs to be elucidated (Boyle et al., 2006).

\subsubsection{MHC class I genes in rhesus macaques}

Compared to the human $M H C$ the rhesus macaque $M H C$ is characterised by a relative large size. While the human MHC encompasses over 3.7 Mb (MHC sequence consortium, 1999) the rhesus macaque $M H C$ spans about 5.3 Mb, which results from the considerable expansion within the MHC class I A and class I B gene regions (Daza-Vamenta et al., 2004) (Figure 4). Rhesus macaques possess two to four $H L A-A$-like (designated as Мати-A) genes varying in the degree of polymorphism. The genes of the Mamu-Al locus are highly polymorphic and are characterised by high transcription levels (so-called "majors"), whereas the Mamu-A2, $-A 3$, and $-A 4$ genes are not markedly polymorphic and are transcribed at low levels ("minors") (Otting et al., 2007). Differential transcription levels have also been described for the Мати- $B$ alleles in rhesus macaques (Otting et al., 2005). The Мати-B region shows substantial copy number variation, which is maintained by duplication and unequal crossingover events, whereas allelic polymorphism is more or less absent for the Мати-B region (Kulski et al., 2004; Otting et al., 2005). Moreover, rhesus macaques possess a B-like sequence, designated as Мати-I, which displays low levels of polymorphism and appears to be present in each haplotype (Urvater et al., 2000). Sequence analysis of a complete rhesus macaque $M H C$ region revealed a haplotype comprising 19 distinct functional Mamu-B like genes (Daza-Vamenta et al., 2004). 


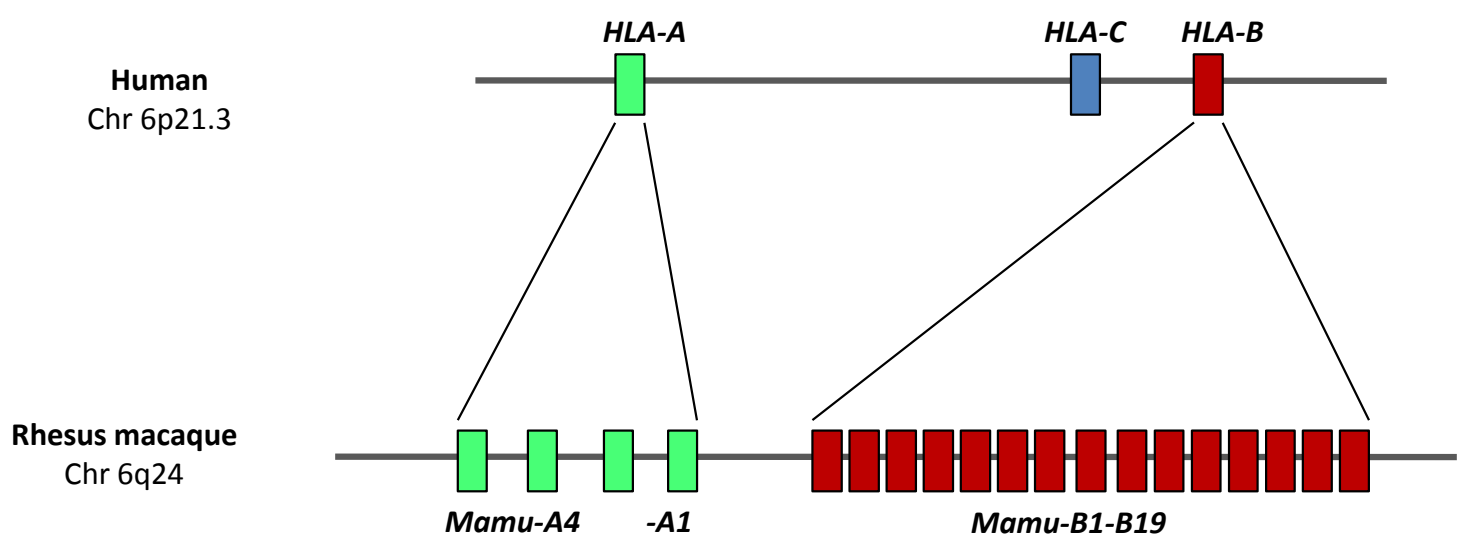

Figure 4 Schematic comparison of the $M H C$ class $I$ region in humans and rhesus macaques.

The chromosomal localization of the $M H C$ in humans and rhesus macaques are shown on the left site. The $M H C-A,-B$, and $-C$ genes are highlighted in green, blue, and red, respectively. They represent a single copy gene in human, while the MHC class I A and class I B gene regions are considerably expanded in rhesus macaques (Figure modified from Daza-Vamenta et al., 2004).

All but one of the six functional HLA class I genes have evolutionary equivalents in the rhesus macaque $M H C($ Mamu- $A,-B,-E,-F$, and $-G)$, with the exception of $H L A-C$, for which a corresponding gene is missing (Boyson et al., 1996). It is supposed that $M H C-C$ evolved and differentiated from one of the multiple $M H C-B$ genes observed in Old World monkeys (e.g. rhesus macaques) (Guethlein et al., 2002). In humans $H L A-A,-B$ and $-C$ represent single copy genes with enormous allelic polymorphism, while the rhesus macaque Mamu-A and Мати-B genes have undergone a complex series of segmental duplications and deletions, resulting in a heterogeneous repertoire of up to 20 MHC class I loci per haplotype (Otting et al., 2005; Daza-Vamenta et al., 2004).

\subsection{Immunodeficiency viruses}

With more than 30 million infected people worldwide, the human immunodeficiency virus (HIV) remains a global health problem (UNAIDS, 2008). Even though research has now led to fundamental progress in understanding HIV pathogenesis and immunity to the virus, the development of an effective vaccine still remains challenging. The clinical outcome after HIV infection is diverse, varying from progression to acquired immunodeficiency syndrome (AIDS) within one year to the prolonged control of viral replication in the absence of antiviral therapy for more than two decades. Such long-surviving individuals are generally termed long-term non-progressors (LTNP) or elite controllers (EC). They are defined as HIV-infected 
individuals, who maintain low or undetectable plasma viral loads $(<50$ copies $/ \mathrm{ml})$ for more than one year without antiretroviral therapy (Bashirova et al., 2011; Walker, 2007).

Simian immunodeficiency virus (SIV)-infected rhesus macaques provide important animal models to study HIV infections. SIV and the closely related HIV-1 and HIV-2 viruses belong to the lentivirus subfamily of retroviruses. SIV is morphologically identical and serologically related to HIV and cytopathic for $\mathrm{CD}^{+} \mathrm{T}$ cells. Additionally, the developed syndromes and the temporally diverse progression to AIDS of SIV-infected rhesus macaques are remarkably similar to human HIV-1 infection (Chakrabarti et al., 1987; Franchini et al., 1987; McClure et al., 1989). Likewise similar to human HIV-1 infection, the outcomes of SIV infection in rhesus macaques are variable, ranging from spontaneous control of plasma viraemia (ECs, about 5-20\% of infected individuals) to uncontrolled viraemia and rapid disease course (fast progressors, about 5-10 \%) (Figure 5). These different outcomes during persistent viraemia in experimentally SIV-infected rhesus macaques suggest that already unique factors of hostvirus interactions during early infection play a critical role.

The main stages of an HIV/SIV infection are divided in the eclipse phase, acute infection, chronic infection, and AIDS (Figure 5). The first phase is the eclipse phase following infection during which plasma viraemia is low. The acute phase starts about two weeks post infection, at that time the virus reaches draining lymph nodes and becomes detectable in plasma (log phase). Rapid viral replication in activated $\mathrm{CD}^{+} \mathrm{T}$ cells and systemic spread lead to an exponential increase in plasma viral load, which reaches its peak 3 to 4 weeks post infection (Fiebig et al., 2003). Simultaneously, $\mathrm{CD}^{+} \mathrm{T}$ cells are activated in virtually all patients, playing an important role in the containment of viral levels (Allen et al., 2005). Latent viral reservoirs are now established, making an extermination of the virus almost impossible. This peak of plasma viraemia is followed by a decrease to a comparatively stable level, called "set point", and defines the end of the acute phase about 12 weeks post infection. The chronic phase of infection can last for years and is characterised by gradual increase in viral load and decrease of $\mathrm{CD}^{+} \mathrm{T}$ cell counts. The progressive loss of $\mathrm{CD} 4^{+} \mathrm{T}$ cells $(\leq 200$ cells/ml) ultimately leaves affected individuals mortally susceptible to opportunistic infections (AIDS). 


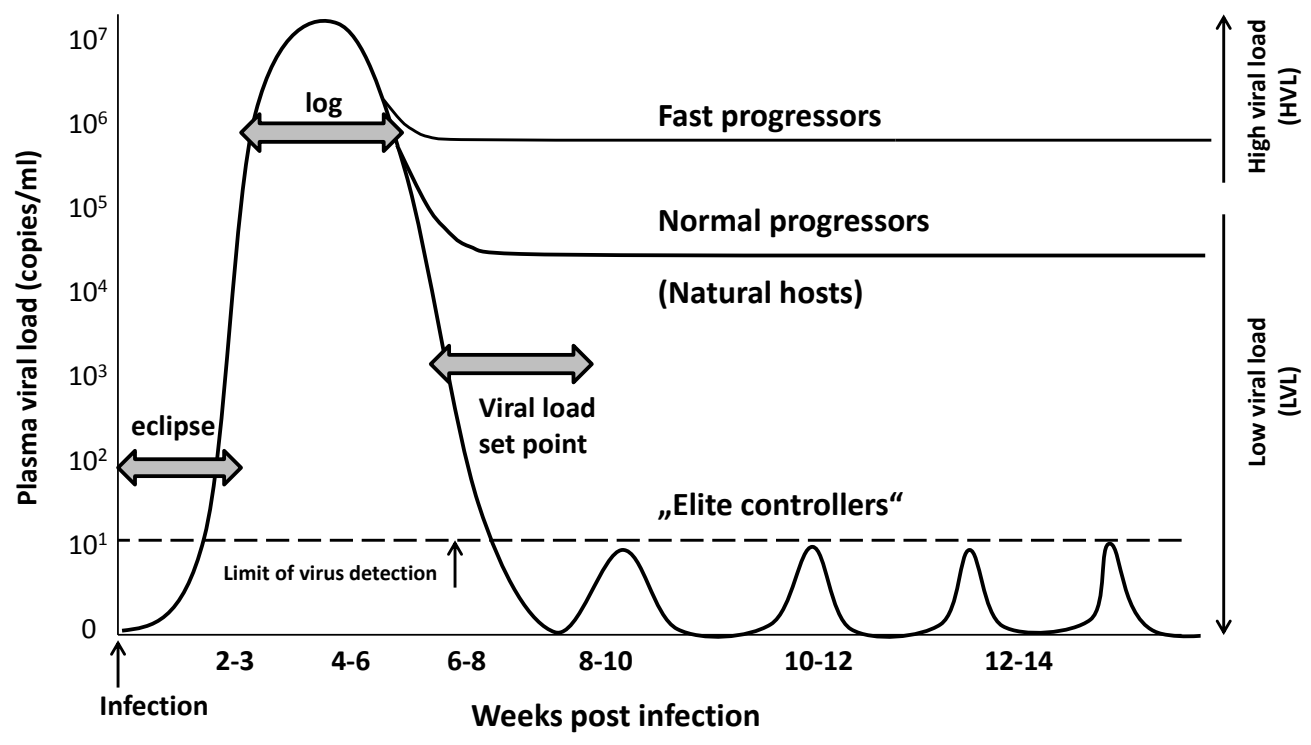

Figure 5 Course of SIV disease

The graph shows the different stages of viraemia in SIV-infected rhesus macaques (modified from Ansari et al., 2011). The first phase is the eclipse phase followed by the log phase during which plasma viraemia reaches its peak. The viral load at set point comprises the phase when the viral load appears to stabilize to varying levels. About one half of SIV-infected rhesus macaques shows high levels of plasma viraemia (HVL), in which $5-10 \%$ are additionally classified as fast disease progressors. The second half shows normal or low levels of viral replication (LVL), in which 5-20\% spontaneously control plasma viraemia and are designated "elite controllers". Natural host establish normal levels of viral loads but they do not progress towards AIDS (Ansari et al., 2011).

The lentiviruses that cause immunodeficiency in humans and Asian macaques originated from cross-species transmission of viruses that originated from viruses that naturally infect African non-human primates (Hahn et al., 2000). Natural hosts for SIV develop plasma viral loads that frequently exceed levels seen in non-natural hosts (Figure 5) and do not exhibit superior cellular control of viraemia, compared to HIV-1 infected humans or SIV-infected rhesus macaques. However, they generally do not progress to AIDS (McClure et al., 1989), suggesting that they have coevolved with the SI-viruses to avoid disease progression. Importantly, immunological control like a virus-specific $\mathrm{T}$ cell and $\mathrm{B}$ cell response is not responsible for the lack of disease progression in natural hosts (Brenchley et al., 2010). Studies in SIV-infected disease-resistant sooty mangabeys have shown that depletion of either $\mathrm{CD}^{+}$or $\mathrm{CD}^{+} \mathrm{T}$ cells failed to lead to any detectable signs of disease (Barry et al., 2007; Klatt et al., 2008). Moreover, studies reported data on higher frequencies of NK cells with considerably higher levels of NK cell activity in SIV-infected sooty mangabeys, compared to non-natural hosts (Pereira et al., 2008; Powell et al., 1989; Ansari et al., 2011). Thus, these data confirm the important role of the innate immune system and mainly of NK cells to contribute to the disease resistant state in sooty mangabeys. 


\subsubsection{NK cells and KIRs in HIV/SIV infection}

NK cells have been established as an important effector of innate immunity in a variety of viral infections including hepatitis C virus (Khakoo et al., 2004), human papillomavirus (Carrington et al., 2005), and HIV/SIV (Alter and Altfeld, 2009; Bostik et al., 2010). Host immune responses to diverse viral infections show very similar patterns, including early induction of type 1 interferons secreted by DCs, increased IL-15 expression, and proliferation of NK cells. The innate immune response is followed by adaptive immunity, like cytokine release and a rapid proliferation of T cells (Biron et al., 1999). The same progression of an antiviral immune response is also observed in HIV-1 infection, indicating that this infection does not alter the kinetics of immune response. NK cells belong to the innate immune system and have the potential to act immediately following HIV infection. They can act directly by cytotoxic activity or indirectly by cytokine release to prime the adaptive immune response (Biron et al., 1999; Cooper et al., 2001). The considerable role of NK cells in early viral control was confirmed by work in natural hosts of SIV (sooty mangabeys), which were shown to exhibit higher frequencies of highly active NK cells. Moreover, NK cell expansion following SIV infection was more rapid compared to infected rhesus macaques (Pereira et al., 2008). Higher NK cell activity was also observed among HIV-infected LTNPs (O`Connor et al., 2007), indicating the importance of NK cells in early viral control.

Genome-wide association studies in HIV-1 disease provided further support for NK cells in the control of disease progression (Fellay et al., 2007). The study identified two particular SNPs (single nucleotide polymorphisms), which are directly linked to MHC class I alleles. One SNP marked $H L A-B * 57$ and the second SNP was located upstream of $H L A-C$ and is thought to be related to increased HLA-C expression, which is supposed to be not attributable to any specific $H L A-C$ allele. HLA-C serves as the main ligand for KIRs of the KIR2D family, indicating that this protective effect in HIV infection is NK cell-dependent via interaction between these two molecules.

During acute HIV-1 infection, cytolytic CD56 ${ }^{\mathrm{dim}}$ NK cells were shown to be considerably expanded, while on-going viral replication results in a deletion of CD56 ${ }^{\mathrm{dim}} \mathrm{NK}$ cells with simultaneous increase of functionally anergic CD56 $\mathrm{CD}^{-} 6^{+} \mathrm{NK}$ cells (Alter et al., 2005; Mavilio et al., 2005). These data emphasize the important role of KIRs in early HIV-1 infection, which are expressed on abundant levels on the surface of CD56 ${ }^{\mathrm{dim}} \mathrm{NK}$ cells (Cooper et al., 2001).

In the last years multiple epidemiological studies in humans have demonstrated associations between $K I R$ and MHC class I gene combinations and HIV-1 disease progression. Particularly 
KIR3DL1 and KIR3DS1, in context with $H L A-B$ alleles within the Bw4 superfamily that contain an isoleucine at position 80 (Bw4-80I), like $H L A-B * 57$ and $H L A-B * 27$, have received the most attention (Alter et al., 2007a; Martin et al., 2002; Martin et al., 2007). The first epidemiological study by Martin et al. (2002) showed that HIV-1-infected individuals expressing KIR3DS1 in context with HLA-Bw4 progressed significantly slower to AIDS than individuals having only one or neither of these two alleles. Interestingly, the physical interaction between the activating KIR3DS1 receptor and its putative HLA-Bw4 ligand could not be demonstrated by now. Nevertheless, KIR3DS1 positive NK cells were reported to inhibit HIV-1 replication more strongly in HLA-Bw4-80I expressing target cells, compared to KIR3DS1 negative NK cells (Alter et al., 2007a), suggesting an interplay between these two molecules. In addition, both KIR3DS1- and KIR3DL1-positive NK cells are elevated during acute HIV-1 infection in the presence of their putative HLA-B Bw4-80I ligands (Alter et al., 2009). Interestingly, also specific inhibitory allotypes of KIR3DL1 in combination with HLABw4 ligands were shown to be protective in HIV-1 disease (Martin et al., 2007). Varying expression levels of KIR3DL1 on the cell surface of NK cells correlate with different functional capacities of NK cells (Yawata et al., 2006). In particular KIR3DL1 alleles encoding for receptors that are expressed on high levels are associated with delayed progression to AIDS (Martin et al., 2007). The protective effect of highly expressed inhibitory KIR receptors might result from interaction with MHC class I ligands during development. Studies in mice and humans have shown that interaction between MHC class I-specific NK cell receptors and self-MHC class I during development confers a signal that results in fully competent peripheral NK cells. This model was called "licencing" (Kim et al., 2005; Anfossi et al., 2006). In contrast, in the absence of an inhibitory receptor-self MHC class I ligand interaction, NK cells are unlicensed and remain functionally inert. Strong inhibitory interactions between NK cells that express KIR3DL1 at high levels and HLA-Bw4 ligands during development might result in more cytolytic NK cells, able to respond more vigorous under appropriate conditions, like HIV-1 infection (Martin et al., 2007). Despite genetic associations between KIR3DL1/KIR3DS1 and HIV disease progression and few functional data the functional basis for the mediated protection in human HIV-1 infection still remains largely unknown.

Recently, Alter et al. (2011) have described KIR-associated amino-acid polymorphisms in the HIV-1 sequence of chronically infected individuals, suggesting that NK cells can directly mediate antiviral pressure in humans. They showed that HIV-1 sequence polymorphisms reduce the antiviral activity of KIR-expressing NK cells by modulating the interaction of 
inhibitory KIRs to infected cells and thereby enabling HIV-1 to escape the NK cell-mediated immunity.

In humans, the $H L A$ class $I$ alleles $H L A-B * 57$ and $H L A-B * 27$ are associated with improved protection and are over-represented in LNTPs (Carrington et al., 2008; Migueles et al., 2000). In rhesus macaques the $M H C$ class $I$ alleles Mamu-AI*001, Мати-B*17 and $-B * 08$ were shown to have a similar protective effect in SIV infection (Mühl et al., 2002; Yant et al., 2006; Loffredo et al., 2007). Moreover, certain rhesus macaque MHC class I haplotypes, which were associated with susceptibility and resistance to SIV were identified (Sauermann et al., 2008). In the last years the knowledge of rhesus macaque KIR genes was fundamentally improved by several studies (Blokhuis et al., 2010; Kruse et al., 2010; Blokhuis et al., 2011). Although rhesus macaques provide important animal models to study HIV infections and the role of MHC class I genes in SIV pathogenesis is already intensively studied, less is known about the influence of $K I R$ gene expression on SIV disease progression. By now, only two KIR3DL05 alleles were identified to be associated with high levels of viral replication in a cohort of 38 SIV-infected rhesus macaques (Bostik et al., 2009). However, knowledge of KIR genes in rhesus macaques that might have an influence on disease outcome in SIV infection is crucial to evaluate the rhesus macaque AIDS model. Work in rhesus macaques provides several advantages, like the ability to use sequence identical viruses and the easy access to pre and acute phase samples, while pre infection data are missing in HIV studies. Investigation of the mechanisms controlling a SIV infection in ECs and in natural host may provide a key model to understanding the factors that mediate protective immunity.

\subsection{Aims of the study}

Based on physiological similarities to human, rhesus macaques provide important animal models of many human infectious and autoimmune diseases where KIR and MHC class I molecules might be involved. In humans, certain combinations of KIRs and HLA class I ligands are associated with lower viral load and slower progression to AIDS, however, such associations are currently not known for rhesus macaques, but would be crucial for full evaluation of the animal model.

Due to these restricted information a key element in this approach is to use 454 next generation sequencing of cDNA-PCR amplicons for comprehensive analysis of $K I R$ and MHC class $I$ transcription in rhesus macaques. SIV-infected rhesus macaques were studied here to identify transcribed KIR and MHC class I genes that are associated with SIV disease 
pathogenesis. Samples of different points in time over the course of SIV infection were analysed to obtain information on SIV-dependent changes in KIR transcription. Moreover, comprehensive analyses of a large panel of immune genes and cell markers using a microfluidic-based real-time PCR approach was performed to identify phenotypic differences between individuals, which might contribute to different disease outcomes.

Overall, this study aimed in achieving advanced knowledge of rhesus macaque KIR gene transcription in SIV infection with respect to different outcomes during persistent viraemia, and to identify the influence of experimental SIV infection on KIR gene transcription over the course of disease. 


\section{Material and Methods}

\subsection{Material}

\subsubsection{Animals}

Samples of peripheral blood mononuclear cells (PBMCs) utilized for the studies reported herein were obtained from a group of outbred rhesus macaques of Indian origin (Macaca mulatta $(\mathrm{mm}) ; \mathrm{n}=52)$, infected intravenously with 200 TCID50 (tissue culture infective dose 50) of either SIVmac239 or SIVmac251. Based on the average viral loads of each monkey at viral load "set point", the samples were divided into two groups: those that represented monkeys with high viral load (HVL $>10^{6}$ viral copies/ $\mathrm{ml}$ of plasma, $\mathrm{n}=28$ ) and those with low viral load $\left(\mathrm{LVL}<10^{5}\right.$ viral copies/ml of plasma, $\left.\mathrm{n}=24\right)$. Samples were obtained before infection (pre), approximately 2 weeks after infection (acute), and 12 weeks after infection at the earliest (chronic). Samples and all information on viral loads were provided by Professor Aftab Ansari (Department of Pathology, Emory University School of Medicine, Atlanta).

Table 1 Animals and SIV status

\begin{tabular}{|c|c|c|c|c|}
\hline \multirow[t]{2}{*}{ Animal } & \multirow[t]{2}{*}{ virus strains } & Peak & Set point & \multirow[t]{2}{*}{ survival time } \\
\hline & & \multicolumn{2}{|c|}{ viral loads (copies/ml) } & \\
\hline \multicolumn{5}{|c|}{ HVL cohort } \\
\hline $\mathrm{mm1}$ & SIVmac239 & 24.000 .000 & 6.000 .000 & 26 weeks \\
\hline $\mathrm{mm} 3$ & SIVmac239 & 29.000 .000 & 28.841 .600 & 20 weeks \\
\hline $\mathrm{mm5}$ & SIVmac239 & 15.000 .000 & 8.763 .600 & 21 weeks \\
\hline $\mathrm{mm6}$ & SIVmac239 & 24.000 .000 & 6.912 .600 & 57 weeks \\
\hline $\mathrm{mm7}$ & SIVmac239 & 12.000 .000 & 1.200 .000 & 70 weeks \\
\hline $\mathrm{mm} 8$ & SIVmac239 & 53.000 .000 & 31.426 .100 & 15 weeks \\
\hline $\mathrm{mmg}$ & SIVmac239 & 66.000 .000 & 27.000 .000 & 17 weeks \\
\hline mm10 & SIVmac239 & 12.000 .000 & 6.200 .000 & 59 weeks \\
\hline $\mathrm{mm} 16$ & SIVmac239 & 33.000 .000 & 18.000 .000 & 67 weeks \\
\hline mm17 & SIVmac239 & 35.000 .000 & 36.831 .700 & 16 weeks \\
\hline mm18 & SIVmac239 & 7.000 .000 & 3.434 .500 & 56 weeks \\
\hline mm19 & SIVmac251 & 10.800 .000 & 2.950 .000 & 151 weeks \\
\hline $\mathrm{mm} 22$ & SIVmac239 & 104.000 .000 & 2.000 .000 & 33 weeks \\
\hline $\mathrm{mm} 23$ & SIVmac251 & 6.000 .000 & 2.640 .000 & 67 weeks \\
\hline $\mathrm{mm} 24$ & SIVmac239 & 36.000 .000 & 3.300 .000 & 38 weeks \\
\hline $\mathrm{mm} 25$ & SIVmac251 & 21.000 .000 & 4.000 .000 & 14 weeks \\
\hline $\mathrm{mm} 26$ & SIVmac239 & 8.900 .000 & 5.041 .100 & 33 weeks \\
\hline $\mathrm{mm} 27$ & SIVmac251 & 6.730 .000 & 2.000 .000 & 54 weeks \\
\hline $\mathrm{mm} 29$ & SIVmac239 & 2.900 .000 & 1.500 .000 & 56 weeks \\
\hline $\mathrm{mm} 33$ & SIVmac239 & 5.250 .000 & 3.000 .000 & 50 weeks \\
\hline mm39 & SIVmac239 & 5.250 .500 & 331.491 & 26 weeks \\
\hline $\mathrm{mm} 40$ & SIVmac239 & 41.282 .900 & 1.868 .060 & 30 weeks \\
\hline $\mathrm{mm} 42$ & SIVmac239 & 6.932 .800 & 2.129 .460 & 30 weeks \\
\hline $\mathrm{mm} 45$ & SIVmac239 & 43.477 .000 & 1.261 .130 & 30 weeks \\
\hline $\mathrm{mm} 47$ & SIVmac239 & 23.478 .600 & 1.912 .690 & 30 weeks \\
\hline
\end{tabular}




\begin{tabular}{|c|c|c|c|c|}
\hline \multirow[t]{2}{*}{ Animal } & \multirow[t]{2}{*}{ virus strains } & Peak & Set point & \multirow[t]{2}{*}{ survival time } \\
\hline & & \multicolumn{2}{|c|}{ viral loads (copies/ml) } & \\
\hline $\mathrm{mm} 49$ & SIVmac239 & 4.035 .400 & 498.604 & 30 weeks \\
\hline mm51 & SIVmac239 & 9.094 .900 & 3.673 .200 & 38 weeks \\
\hline mm52 & SIVmac239 & 2.752 .740 & 287.406 & 60 weeks \\
\hline \multicolumn{5}{|c|}{ LVL cohort } \\
\hline $\mathrm{mm} 2$ & SIVmac239 & 19.000 .000 & 11.000 & 19 months \\
\hline $\mathrm{mm} 4$ & SIVmac239 & 1.900 .000 & 70.184 & 16 months \\
\hline $\mathrm{mm11} *$ & SIVmac239 & 1.600 .000 & 800 & $>3$ years \\
\hline $\mathrm{mm12} *$ & SIVmac239 & 31.000 .000 & 2.000 & 6 month** \\
\hline $\mathrm{mm13} *$ & SIVmac239 & 6.700 & 1.000 & 23 months \\
\hline mm14 & SIVmac239 & 370.000 & 10.000 & 3 years \\
\hline mm15 & SIVmac239 & 78.000 & 20.000 & 2.5 years \\
\hline $\mathrm{mm} 20$ & SIVmac239 & 4.200 & 20 & 2 years \\
\hline $\mathrm{mm} 21$ & SIVmac239 & 330.000 & 4.000 & 2 years \\
\hline mm28* & SIVmac239 & 7.600 .000 & 2.770 & 6 month** \\
\hline $\mathrm{mm} 30 *$ & SIVmac239 & 5.600 .000 & 1.100 & 6 month** \\
\hline $\mathrm{mm} 31 *$ & SIVmac239 & 160.000 & 300 & $>3$ years \\
\hline $\mathrm{mm} 32 *$ & SIVmac239 & 340.000 & 700 & 1 year \\
\hline mm34 & SIVmac251 & 300.000 & 4.000 & 17 months \\
\hline mm35* & SIVmac239 & 2.300 .000 & 300 & $>3$ years \\
\hline $\mathrm{mm} 36^{*}$ & SIVmac239 & 5.700 .000 & 9.100 & 6 month** \\
\hline mm37* & SIVmac239 & 870.000 & 20.000 & $>3$ years \\
\hline mm38* & SIVmac251 & 1.110 .000 & 1.500 & $>3$ years \\
\hline mm41 & SIVmac239 & 4.743 .000 & 1.623 & 17 months \\
\hline $\mathrm{mm} 43$ & SIVmac239 & 2.812 .640 & 166 & 15 months \\
\hline $\mathrm{mm} 44^{*}$ & SIVmac239 & 32.588 .000 & 166 & 17 months \\
\hline mm46 & SIVmac239 & 2.114 .780 & 12.994 & 16 months \\
\hline $\mathrm{mm} 48$ & SIVmac239 & 6.242 .800 & 39.177 & 17 months \\
\hline $\mathrm{mm} 50$ & SIVmac239 & 2.664 .650 & 12.232 & 17 months \\
\hline RSt4 & uninfected & \multicolumn{3}{|c|}{ RNA samples from NK cells, CD8+ cells, and PBMCs } \\
\hline RFn10 & uninfected & \multicolumn{3}{|c|}{ RNA samples from NK cells, CD8+ cells, and PBMCs } \\
\hline
\end{tabular}

* Elite Controller

** Sacrificed quickly because animal was controlling

\subsubsection{Equipment}

\begin{tabular}{l|l|l} 
Equipment & Version & Manufacturer \\
\hline Autoclave & Varioklav® 400E & H+P Labortechnik \\
Bead Counter & GS Junior & Roche \\
Bioanalyzer & 2100 & Agilent \\
BioMark ${ }^{\mathrm{TM}}$ HD System & $5810 \mathrm{R}$ & Fluidigm \\
Centrifuges & $5415 \mathrm{D}$ & Eppendorf \\
Eppendorf \\
Centrifuge Rotors & F45-24-11 & Eppendorf \\
Eppendorf
\end{tabular}




\begin{tabular}{|c|c|c|}
\hline Equipment & Version & Manufacturer \\
\hline Counterweight and Adaptor & GS Junior & Roche \\
\hline Electrophoresis chamber & Horizon 58 & Gibco BRL \\
\hline IFC Controller & MX & Fluidigm \\
\hline Fluorometer & QuantiFluor & Promega \\
\hline Fridge $4^{\circ} \mathrm{C}$ & Profi line & Liebherr \\
\hline Freezer $-20^{\circ} \mathrm{C}$ & Premium & Liebherr \\
\hline Gel imager & Gel Jet Imager 2000 & Intas \\
\hline Magnet stirrer & M32 & GLW \\
\hline Magnet & DYNAL Bead Separations & Invitrogen \\
\hline Microwave & Micromat & AEG \\
\hline PCR Work Station & Captair Bio & Erlab \\
\hline \multirow[t]{8}{*}{ Pipettes } & 2-20 $\mu 1$ peqPETTE & peqlab \\
\hline & 20-200 $\mu 1$ peqPETTE & peqlab \\
\hline & 100-1000 $\mu 1$ peqPETTE & peqlab \\
\hline & 0.5-10 $\mu 1$ Research Plus & Eppendorf \\
\hline & Multichannel 0.5-10 $\mu 1$ & Eppendorf \\
\hline & Multichannel 10-100 $\mu 1$ & Eppendorf \\
\hline & Multichannel 20-200 $\mu 1$ & peqlab \\
\hline & Multipette plus & Eppendorf \\
\hline $\mathrm{pH}$ meter & pH 535 Multi Cal $\circledast$ & WTW \\
\hline Power supply & Etron-S & Etron \\
\hline Scale & BP $310 \mathrm{~s}$ & Sartorius \\
\hline Sequencer & GS Junior & Roche \\
\hline Shaker & Labquake & Thermo Scientific \\
\hline Spectrophotometer & Nanodrop ND-1000 & peqlab \\
\hline \multirow[t]{3}{*}{ Thermocycler } & GeneAmp PCR System 2700 & Applied Biosystems \\
\hline & Labcycler & Sensoquest \\
\hline & PTC-200 & Bio-Rad \\
\hline Thermomixer & Thermomixer Compact & Eppendorf \\
\hline Vacuum pump & & H. Saur Laborbedarf \\
\hline \multirow[t]{3}{*}{ Vortexer } & L46 & GLT \\
\hline & Ultra turrax ${ }^{\circledR}$ Tube Drive Control & IKA \\
\hline & MS 3 & IKA \\
\hline
\end{tabular}




\subsubsection{Consumables}

\section{Consumables}

2-Propanol

48.48 Control Line Fluid

48.48 Dynamic Array Gene expression

Agarose

Agencourt ${ }^{\circledR}$ AMPure XP®

Assay Loading Reagent (2x)

BioTherm. ${ }^{\text {TM }} 10 \mathrm{x}$ Reaction Buffer

BioTherm. ${ }^{\text {TM }}$ Taq DNA Polymerase

Boric acid

Bromphenol blue

Combitips ${ }^{\circledR}$ Plus (5 ml)

Consumables

Cuvettes (Minicell Adapter)

DEPC

DNA Binding Dye Sample Loading Reagent (20x)

DNA Ladder 100bp Plus

DNA Suspension Buffer

dNTP

Ethanol

Ethidiumbromid

Ethylendiamintetraacetat (EDTA)

Exonuclease I

Falcon Tube (15ml)

Falcon Tube $(50 \mathrm{ml})$

FastStart High Fidelity PCR System, dNTPack

Filter Tips $(0.5-20 \mu \mathrm{l})$

Filter Tips $(1000 \mu \mathrm{l})$

Filter Tips $(2.5 \mu 1)$

Filter Tips $(5-200 \mu \mathrm{l})$

High Sensitivity DNA Chips

High Sensitivity DNA Reagents

\section{Manufacturer}

Roth

Fluidigm

Fluidigm

Cambrex (Biozym)

Beckman Coulter

Fluidigm

Genecraft

Genecraft

Roth

Sigma

Eppendorf

Manufacturer

Promega

Roth

Fluidigm

Fermentas

TEKnova

Invitrogen

Merck

Roth

Sigma

NEB

Becton Dickinson

Becton Dickinson

Roche

Peqlab

Sarstedt

Biozym

Peqlab

Agilent

Agilent 


\section{Consumables}

Matte Finish Magic ${ }^{\mathrm{TM}}$ Tape

M-MLV 5 x Buffer

M-MLV RT

Moist Cleaning Tissue "Pro-Optic"

Oligo(dT)15-Primer

PCR Film

PCR Plate 96 twin.tec

Precision Wipes

Reaction tube $(1.5 \mathrm{ml})$

Reaction tube $(2 \mathrm{ml})$

Reagent Reservoirs

RNasin

Scalpel

Serological pipette $(10 \mathrm{ml})$

Serological pipette $(50 \mathrm{ml})$

Sodium Hydroxid

Sparkleen 1

SsoFast ${ }^{\mathrm{Tm}}$ EvaGreen qPCR Master Mix with Low ROX

Sucrose

Syringe Kit

Needles

Taqman Preamp Master Mix

Tris

Tubes $2 \mathrm{ml}$

Tween 20

Water (HPLC quality)

Water (Molecular Biology Grade)

$\beta$-Mercaptoethanol
Manufacturer

Scotch

Promega

Promega

Hama PHOTO

Promega

Eppendorf

Eppendorf

Kimberly-Clark Professional

Sarstedt

Roth

VWR

Promega

Braun

Sarstedt

Greiner bio-one

Roth

Fisherbrand

Biotium

Merck

Agilent

WDT

Applied Biosystems

Roth

Roth

Fisher Scientific

Merck

Fisher BioReagents

Sigma 


\subsubsection{Kits}

\begin{tabular}{l|l} 
Kit & Manufacturer \\
\hline emPCR Kit emPCR Reagents (Lib-A) & Roche \\
emPCR Kit Oil and Breaking Kit & Roche \\
emPCR Bead Recovery Reagents & Roche \\
Sequencing Kit Buffers & Roche \\
Sequencing Kit Reagents and Enzymes & Roche \\
Sequencing Kit Packing Beads and Supplement CB & Roche \\
PicoTiterPlate Kit & Roche \\
Syringe Kit & Agilent \\
Gene Expression Installation Kit & Fluidigm \\
QIAquick Gel Extraction Kit & Quiagen \\
RNeasy Plus Mini Kit & Quiagen \\
Quant-iT ${ }^{\text {TM PicoGreen }}{ }^{\circledR}$ dsDNA Assay Kit & Invitrogen
\end{tabular}

\subsubsection{Buffers and Solutions}

\begin{tabular}{|c|c|}
\hline Buffer/Solution & Substance of content \\
\hline \multirow[t]{3}{*}{ Loading buffer (DNA) } & $6.6 \mathrm{~g}$ Sucrose \\
\hline & $0.04 \mathrm{~g}$ Bromphenol blue \\
\hline & dissolve in $10 \mathrm{ml} \mathrm{H}_{2} \mathrm{O}$ \\
\hline \multirow[t]{4}{*}{ TBE Agarose gel (1\%) } & $1 \mathrm{~g}$ Agarose \\
\hline & add $1 \mathrm{x}$ TBE Puffer to $100 \mathrm{ml}$ \\
\hline & heat to $60^{\circ} \mathrm{C}$ and cool down \\
\hline & $5 \mu \mathrm{EtBr}$ \\
\hline \multirow[t]{3}{*}{ TBE buffer $(10 x)$} & $1 \mathrm{M}$ Tris \\
\hline & $0.8 \mathrm{M}$ boric acid \\
\hline & 10 mM EDTA (pH 8.0) \\
\hline \multirow[t]{3}{*}{ TE buffer } & $10 \mathrm{mM}$ Tris \\
\hline & 1 mM EDTA (pH 8.0) \\
\hline & adjust $\mathrm{pH} 7.4$ \\
\hline
\end{tabular}




\subsubsection{Oligonucleotides}

Primers were ordered at Metabion. Each primer was synthesized and HPLC purified. They were delivered lyophilized and dissolved in HPLC purified water to a final concentration of $100 \mathrm{pmol} / \mu 1$. Forward primers (F) initiate the strand synthesis in 5 -3 'direction and reverse primer $(\mathrm{R})$ in $3^{\prime}-5{ }^{\prime}$ direction. The multiplex identifiers (MID) are shown in bold.

Table 2454 cDNA amplicon primer (KIR)

\begin{tabular}{|c|c|}
\hline Name & Sequence $\left(5^{\prime}-3^{\prime}\right)$ \\
\hline KIR-405-F_MID01 & CGTATCGCCTCCCTCGCGCCATCAGACGAGTGCGTAGGTCCCCTGGTGAAATCAG \\
\hline KIR-405-F_MID02 & CGTATCGCCTCCCTCGCGCCATCAGACGCTCGACAAGGTCCCCTGGTGAAATCAG \\
\hline KIR-405-F_MID03 & CGTATCGCCTCCCTCGCGCCATCAGAGACGCACTCAGGTCCCCTGGTGAAATCAG \\
\hline KIR-405-F_MID04 & CGTATCGCCTCCCTCGCGCCATCAGAGCACTGTAGAGGTCCCCTGGTGAAATCAG \\
\hline KIR-405-F_MID05 & CGTATCGCCTCCCTCGCGCCATCAGATCAGACACGAGGTCCCCTGGTGAAATCAG \\
\hline KIR-405-F_MID06 & CGTATCGCCTCCCTCGCGCCATCAGATATCGCGAGAGGTCCCCTGGTGAAATCAG \\
\hline KIR-405-F_MID07 & CGTATCGCCTCCCTCGCGCCATCAGCGTGTCTCTAAGGTCCCCTGGTGAAATCAG \\
\hline KIR-405-F_MID08 & CGTATCGCCTCCCTCGCGCCATCAGCTCGCGTGTCAGGTCCCCTGGTGAAATCAG \\
\hline KIR-405-F_MID09 & CGTATCGCCTCCCTCGCGCCATCAGTAGTATCAGCAGGTCCCCTGGTGAAATCAG \\
\hline KIR-405-F_MID10 & CGTATCGCCTCCCTCGCGCCATCAGTCTCTATGCGAGGTCCCCTGGTGAAATCAG \\
\hline KIR-405-F_MID11 & CGTATCGCCTCCCTCGCGCCATCAGTGATACGTCTAGGTCCCCTGGTGAAATCAG \\
\hline KIR-405-F_MID12 & CGTATCGCCTCCCTCGCGCCATCAGTACTGAGCTAAGGTCCCCTGGTGAAATCAG \\
\hline KIR-1004-R_MID01 & CTATGCGCCTTGCCAGCCCGCTCAGACGAGTGCGTCTTGGTTCAGTGGGTGAAGG \\
\hline KIR-1004-R_MID02 & CTATGCGCCTTGCCAGCCCGCTCAGACGCTCGACACTTGGTTCAGTGGGTGAAGG \\
\hline KIR-1004-R_MID03 & CTATGCGCCTTGCCAGCCCGCTCAGAGACGCACTCCTTGGTTCAGTGGGTGAAGG \\
\hline KIR-1004-R_MID04 & CTATGCGCCTTGCCAGCCCGCTCAGAGCACTGTAGCTTGGTTCAGTGGGTGAAGG \\
\hline KIR-1004-R_MID05 & CTATGCGCCTTGCCAGCCCGCTCAGATCAGACACGCTTGGTTCAGTGGGTGAAGG \\
\hline KIR-1004-R_MID06 & CTATGCGCCTTGCCAGCCCGCTCAGATATCGCGAGCTTGGTTCAGTGGGTGAAGG \\
\hline KIR-1004-R_MID07 & CTATGCGCCTTGCCAGCCCGCTCAGCGTGTCTCTACTTGGTTCAGTGGGTGAAGG \\
\hline KIR-1004-R_MID08 & CTATGCGCCTTGCCAGCCCGCTCAGCTCGCGTGTCCTTGGTTCAGTGGGTGAAGG \\
\hline KIR-1004-R_MID09 & CTATGCGCCTTGCCAGCCCGCTCAGTAGTATCAGCCTTGGTTCAGTGGGTGAAGG \\
\hline KIR-1004-R_MID10 & CTATGCGCCTTGCCAGCCCGCTCAGTCTCTATGCGCTTGGTTCAGTGGGTGAAGG \\
\hline KIR-1004-R_MID11 & CTATGCGCCTTGCCAGCCCGCTCAGTGATACGTCTCTTGGTTCAGTGGGTGAAGG \\
\hline KIR-1004-R_MID12 & CTATGCGCCTTGCCAGCCCGCTCAGTACTGAGCTACTTGGTTCAGTGGGTGAAGG \\
\hline
\end{tabular}


Table 3454 cDNA amplicon primer (MHC class I)

\begin{tabular}{|c|c|}
\hline Name & Sequence $\left(5^{\prime}-3^{\prime}\right)$ \\
\hline GS GSA-MID1-SBT190F & CGTATCGCCTCCCTCGCGCCATCAGACGAGTGCGTGCTACGTGGACGACACG \\
\hline GS GSA-MID2-SBT190F & CGTATCGCCTCCCTCGCGCCATCAGACGCTCGACAGCTACGTGGACGACACG \\
\hline GS GSA-MID3-SBT190F & CGTATCGCCTCCCTCGCGCCATCAGAGACGCACTCGCTACGTGGACGACACG \\
\hline GS GSA-MID4-SBT190F & CGTATCGCCTCCCTCGCGCCATCAGAGCACTGTAGGCTACGTGGACGACACG \\
\hline GS GSA-MID5-SBT190F & CGTATCGCCTCCCTCGCGCCATCAGATCAGACACGGCTACGTGGACGACACG \\
\hline GS GSA-MID6-SBT190F & CGTATCGCCTCCCTCGCGCCATCAGATATCGCGAGGCTACGTGGACGACACG \\
\hline GS GSA-MID7-SBT190F & CGTATCGCCTCCCTCGCGCCATCAGCGTGTCTCTAGCTACGTGGACGACACG \\
\hline GS GSA-MID8-SBT190F & CGTATCGCCTCCCTCGCGCCATCAGCTCGCGTGTCGCTACGTGGACGACACG \\
\hline GS GSA-MID9-SBT190F & CGTATCGCCTCCCTCGCGCCATCAGTAGTATCAGCGCTACGTGGACGACACG \\
\hline GS GSA-MID10-SBT190F & CGTATCGCCTCCCTCGCGCCATCAGTCTCTATGCGGCTACGTGGACGACACG \\
\hline GS GSA-MID11-SBT190F & CGTATCGCCTCCCTCGCGCCATCAGTGATACGTCTGCTACGTGGACGACACG \\
\hline GS GSA-MID12-SBT190F & CGTATCGCCTCCCTCGCGCCATCAGTACTGAGCTAGCTACGTGGACGACACG \\
\hline GS GSB-MID1-SBT367R & CTATGCGCCTTGCCAGCCCGCTCAGACGAGTGCGTTCCCACTTSCGCTGGGT \\
\hline GS GSB-MID2-SBT367R & CTATGCGCCTTGCCAGCCCGCTCAGACGCTCGACATCCCACTTSCGCTGGGT \\
\hline GS GSB-MID3-SBT367R & CTATGCGCCTTGCCAGCCCGCTCAGAGACGCACTCTCCCACTTSCGCTGGGT \\
\hline GS GSB-MID4-SBT367R & CTATGCGCCTTGCCAGCCCGCTCAGAGCACTGTAGTCCCACTTSCGCTGGGT \\
\hline GS GSB-MID5-SBT367R & CTATGCGCCTTGCCAGCCCGCTCAGATCAGACACGTCCCACTTSCGCTGGGT \\
\hline GS GSB-MID6-SBT367R & CTATGCGCCTTGCCAGCCCGCTCAGATATCGCGAGTCCCACTTSCGCTGGGT \\
\hline GS GSB-MID7-SBT367R & CTATGCGCCTTGCCAGCCCGCTCAGCGTGTCTCTATCCCACTTSCGCTGGGT \\
\hline GS GSB-MID8-SBT367R & CTATGCGCCTTGCCAGCCCGCTCAGCTCGCGTGTCTCCCACTTSCGCTGGGT \\
\hline GS GSB-MID9-SBT367R & CTATGCGCCTTGCCAGCCCGCTCAGTAGTATCAGCTCCCACTTSCGCTGGGT \\
\hline GS GSB-MID10-SBT367R & CTATGCGCCTTGCCAGCCCGCTCAGTCTCTATGCGTCCCACTTSCGCTGGGT \\
\hline GS GSB-MID11-SBT367R & CTATGCGCCTTGCCAGCCCGCTCAGTGATACGTCTTCCCACTTSCGCTGGGT \\
\hline GS GSB-MID12-SBT367R & CTATGCGCCTTGCCAGCCCGCTCAGTACTGAGCTATCCCACTTSCGCTGGGT \\
\hline
\end{tabular}




\subsubsection{Real-time PCR assay (Fluidigm)}

The DELTAgene ${ }^{\mathrm{TM}}$ Assay is a custom based qPCR gene expression assay by Fluidigm. The assay was delivered as a 48 forward and reverse primer mix with each primer at a concentration of $100 \mu \mathrm{M}$.

Table 4 DELTAgene Assay for qPCR (gene expression)

\begin{tabular}{|c|c|c|c|}
\hline $\begin{array}{l}\text { Gene } \\
\text { Symbol }\end{array}$ & Design RefSeq & Primer forward $\left(5^{\prime}-3^{\prime}\right)$ & Primer reverse $\left(5^{\prime}-3^{\prime}\right)$ \\
\hline CCR7 & NM_001032884 & GTGGTGGCTCTCCTTGTCA & TGTGGTGTTGTCTCCGATGTAA \\
\hline CD14 & M_001130433 & CTCAGCTGCAACAGACTGAAC & GTCCAGTGCCAGGTTATCCA \\
\hline CD160 & XM_001089019 & AAGTCAGGTATCCGCCTTCA & TTCAGTCCCGTCACTGTGTA \\
\hline CD19 & XM_001103043 & TCCCTTGTGGGCATTCTTCA & TCCTGGTGGGGTCAGTCA \\
\hline CD223 & XM_001108923 & CTGGAGCCTTTGGCTTTCAC & GAGGGTGAATCCCTTGCTCTAA \\
\hline CD226 & NM_001042643 & GTGATGAGATTGACCGTAGCC & AAACTGTCCCTCCAGTCACA \\
\hline CD244 & XM_001117630 & AACCACAGCCCTTCCTTCAA & GAGCAGGGTTCTGGGCTTTA \\
\hline CD27 & XM_001104337 & TTCTCTCCAGACCACCACAC & GGTGCAGTTGCGAATGAGAA \\
\hline CD28 & NM_001042641 & TGAGAAGAGCAATGGAACCA & GGCTTAGAAGGTCCAGGAAA \\
\hline CD30L & XM_001099524 & TCCCAACTCACCTGACAACA & TGGAGCCCTTTTCAGGATACA \\
\hline CD38 & XM_001099851 & GCACGATGCGTCAAGTACA & CCCTTGAATGCATCCCATACAC \\
\hline$C D 3 D$ & XM_001097302 & TGCTTTGCTGGACATGAGAC & CTGATAGACCTGGTCATTCCTCA \\
\hline CD3G & XM_001093643 & GCAGACTCTGTTGCCTAATGAC & GGTTTCCTTGAAGGTGACTGTAC \\
\hline CD4 & NM_001042662 & AAGTTGCATCAGGAAGTGAACC & CCCCACACTTCACAGGTCAA \\
\hline CD40 & XM_001104333 & AAGGTGGCCAAGAAGCCAAA & GCCAGGAAGATCGTCCAGAAAA \\
\hline CD4OLG & NM_001032839 & AGAAAGAAAACAGCTTTGAAATGCA & ACTACTGGCCTCACTTATGACA \\
\hline CD69 & XM_001114683 & TCACCCATGGAAGTGGTCAA & AAAAGCGCACTTCTCAGACC \\
\hline CD70 & XM_001088935 & CCGTGGGAATCTGCTCTCC & TGGGAGGCAATGGTACAACC \\
\hline CD80 & NM_001042642 & GTGCTC & CAGCGTTGCCACTTCTTTCA \\
\hline CD86 & NM_001042644 & TCTGTGTTCTGGAAACTGACA & CTGAGGGTCCTCAAGCTCTA \\
\hline CD8B & XR_010580 & GAAGAGAGGGTGC & GAGGGTGATGGGGCTACAA \\
\hline CD94 & NM_001032828 & CAGGACCCAACATAGAACTCCA & CAGTTGCATCGGTACCCAAC \\
\hline CD96 & XM_001102509 & AGGAGCTCCACCACAATCAA & ACCAAGACATCCGTGGAGTTA \\
\hline CTLA4 & NM_001044739 & ATGGGCATAGGCAATGGAAC & GCAAGGATCCAGAGGAGGAA \\
\hline FASLG & NM_001032838 & GATTGGGACTGGGGATGTTTCA & GCTGTATGCTTCTGGCTGGTA \\
\hline FCER1G & XM_001115585 & CAGCAGTGGTCTTGCTCTTAC & AGGAGGGTGAGGACAATTCC \\
\hline FCGR3 & NM_001048248 & TCCTCCCAACTGCTCTGCTA & TTCTCGAGCACCCTGTACCA \\
\hline GAPDH & NM_001195426 & CAGAACATCATCCCTGCCTCTA & CCAGTGAGCTTCCCGTTCA \\
\hline GUSBB & XM_001087699 & AGATCCAACTATGCAGCAGACAA & CCAGGTGCCCGAAGTCATTA \\
\hline GZMB & XM_001114420 & TCCCGCTTAGGCCGTTATTAC & TACACACAAGGGGTCCTCCA \\
\hline HCST & NM_001032835 & GAGATGGCAAAGTCTACATCAACA & ATGAGAGGGTCGGAAGTCAAA \\
\hline HPRT1 & XM_001097691 & ACGATGCAGACTTTGCTTTCC & CACTTCGTGGAGTCCTTTTCAC \\
\hline
\end{tabular}




\begin{tabular}{|c|c|c|c|}
\hline $\begin{array}{l}\text { Gene } \\
\text { Symbol }\end{array}$ & Design RefSeq & Primer forward $\left(5^{\prime}-3^{\prime}\right)$ & Primer reverse $\left(5^{\prime}-3^{\prime}\right)$ \\
\hline HSP90AB1 & XR_012134 & GAACCATTGCCAAGTCTCGTAC & TGTCTGCACCAGCCTAAACA \\
\hline IL17RA & XM_001102483 & TCGAATGGACACTGCAGACA & GTTCAGCTGCAGGACAGATAAC \\
\hline IL2RA & NM_001032917 & TCCTGGGACAACCAATGTCAA & TGTTCTTCAGGTTGAGGTGTCA \\
\hline IL7R & XM_001092582 & TGAGGACCCAGATGTCAACA & ACTCAGGCACTTTACCTCCA \\
\hline KLRG1 & XM_001114131 & TGACTACGGACCACAGCAA & AGTCAGAAGCCCCAAAGCTA \\
\hline LAMP1 & XM_001087801 & ACTTCTCTGCTGCCTTCTCA & GCATCTGATGGCAGGTCAAA \\
\hline NKG2A & NM_001032829 & TCGCAGGATTTTCAAGGGAA & TGAGCTTCTCTGGAACTGAC \\
\hline NKG2C & NM_00 & AGAGAGTTTGCTGGCCTGTA & ACACCAATCCATGAGGAAGGTA \\
\hline NKG2D & NM_001032889 & AATGGATCTTGGCAGTGGGAA & ACAGTCTCCCTTCTGCATTTCA \\
\hline NKp30 & NM_001042640 & GGGCTGGATTCTATGCTGTCA & GCAGTGTGTTCCCATGTGAC \\
\hline NKp44 & XM_001082574 & TCTGTGGACTCCTCGTAGTCA & GAGCTCCATCAGGGTTTTCCA \\
\hline NKp46 & NM_001032891 & AACCGATCATCAGGGCTGAA & TCCATAACTTCCCTGGCAACA \\
\hline NKp80 & NM_001032961 & GGCTTTGATCTCCCTGATCCTA & TGCTCGGTGGTATTTGAATGAC \\
\hline NKR-P1 & XM_002798482 & ATGCAGTGTGGACATTCAAC & TCTCTTGGACTTGCTTCCAA \\
\hline PRF1 & XM_001107909 & ACAGCTTCAGCACTGACACA & GCTGGGTGGAGGCATTGAA \\
\hline TYROBP & NM_001032867 & ACCCGGAAACAGCGTATCA & TTGAGGTCGCTGTAGACATCC \\
\hline
\end{tabular}

\subsubsection{Database and Software}

- 2100 Expert Software (Version: B.02.08.SI648; Bioanalyzer)

- Biomark Data Collection Software

- Fluidigm Real-Time PCR Analysis Software

- GS Junior System Software v2.5

- GS Junior Sequencer

○ GS Run Processor

- GS Run Browser

- Graphpad Prism 5

- BLAST (http://blast.ncbi.nlm.nih.gov/)

- IPD KIR database (http://www.ebi.ac.uk/ipd/kir; 2. April 2011)

- IPD MHC database (http://www.ebi.ac.uk/ipd/mhc/nhp/index.html; Dec. 2011)

- Graphpad calculator (http://www.graphpad.com/quickcalcs/contingency1.cfm) 


\subsection{Methods}

\subsubsection{Total RNA isolation}

Total RNA was isolated from peripheral blood mononuclear cells (PBMCs) stored in RNAlater. $250 \mu 1$ RNAlater solution including PBMCs was mixed with $750 \mu 1$ RLT Lysis Buffer (RNeasy Plus Mini Kit; Quiagen) and $10 \mu 1$ ß-mercaptoethanol. Further steps were performed according to the manufacturer's instructions.

\subsubsection{RNA quantification}

The quantity and quality of total RNA was determined by UV absorbance (A260) and A260/A280 ratio ( 1.9-2.0) by using the NanoDrop 1000 (Peqlab).

\subsubsection{Reverse transcription}

Total RNA was reversed transcribed to complementary DNA (cDNA) by using the M-MLV RT (Moloney Murine Leukemia Virus Reverse Transcriptase). A maximum of $1.5 \mu \mathrm{g}$ total RNA was mixed with $1 \mu \mathrm{l}$ Oligo(dT $)_{15}$ Primer $(0.5 \mu \mathrm{g} / \mu \mathrm{l})$ and filled up to a volume of $15 \mu \mathrm{l}$ with RNase free water (Qiagen). Denaturation of RNA secondary structures was performed by incubation at $70^{\circ} \mathrm{C}$ for $5 \mathrm{~min}$ and immediately chilled on ice to let the primer anneal to the RNA. $5 \mu \mathrm{l}$ (5x M-MLV Buffer), $0.5 \mu \mathrm{l}$ dNTPs (25mM each), $0.7 \mu \mathrm{l}$ RNasin (40 U/ $\mu \mathrm{l})$ and $1 \mu \mathrm{l}$ M-MLV RT $(200 \mathrm{U} / \mu \mathrm{l})$ were added and filled up with RNase free water to a volume of $25 \mu$. The RT reaction was extended at $42^{\circ} \mathrm{C}$ for one hour followed by a $70^{\circ} \mathrm{C}$ step for $15 \mathrm{~min}$ to inactivate the enzyme.

\subsubsection{Amplicon preparation by Polymerase Chain Reaction (PCR)}

The PCR technique is used to selectively amplify a specific region of a DNA strand located between two at least partially known DNA regions. The basic reaction mixture contains a heat-stable DNA-polymerase (Taq DNA polymerase), two primers, dNTPs and DNA target containing the DNA region to be amplified.

Amplicons used for next generation sequencing have to fulfil the quality criteria of the 454 GS Junior System (Roche). A schematic overview of forward and reverse amplicon primers are shown in Figure 6. The 5 -portion is a 25 -mer, which is required for binding to the DNA 
Capture Beads during emulsion PCR (emPCR), and for annealing the emPCR Amplification Primers and the Sequencing Primers (red and purple). This sequence ends with the sequencing key "TCAG" (blue underlined) used for amplicon sequencing. Two kinds of such primers allow for the directional sequencing of the target sequence from either end. Finally, a 10-bp tag (MID, yellow) identifying an individual was added between the sequencing key and the template-specific sequence. 12 different MIDs (sequences of the standard 454 GS Junior MID set) were used for barcoding amplicons and allow for sequencing up to 12 individuals within one sequencing run. The 3 -portion contains the target specific sequence. Primers are listed in Table 2 and Table 3.

Forward primer (Primer A):

5`-CGTATCGCCTCCCTCGCGCCATCAG- $\{$ MID $\}$-template-specific sequence-3`

Reverse primer (Primer B):

5`-CTATGCGCCTTGCCAGCCCGCTCAG-\{MID\}-template-specific sequence-3`

Figure 6 Nucleotide sequences of the 454 GS Junior amplicon primers.

The 5-portion is a 25-mer (red, purple), whose sequence is determined by the 454 GS Junior requirements and ends with a key (underlined blue) used for amplicon sequencing. This part is followed by a barcode sequence (MID, yellow) and the target-specific sequence (green).

\subsubsection{KIR CDNA-PCR amplicon preparation}

Primers used for amplification of rhesus macaque KIR amplicons (Table 2) were designed as described previously (Moreland et al., 2011). cDNA-PCR amplicons spanning 623 bp of the D1 and D2 domain of KIR genes (see Figure 3), were synthesized using the FastStart High Fidelity PCR System. The PCR reaction mix was prepared according to the protocol instructions (454 GS Junior, Roche). For each cDNA sample forward and reverse primers with identical MIDs were used.

PCR conditions:

\section{Temperature Time}

$\left.\begin{array}{lll}\text { Initial denaturation } & 94^{\circ} \mathrm{C} & 3 \mathrm{~min} \\ \text { Denaturation } & 94^{\circ} \mathrm{C} & 15 \mathrm{sec} \\ \text { Annealing } & 61^{\circ} \mathrm{C} & 45 \mathrm{sec} \\ \text { Elongation } & 72^{\circ} \mathrm{C} & 1 \mathrm{~min}\end{array}\right]-35$ cycles




\subsubsection{MHC class I cDNA-PCR amplicon preparation}

Primers used for amplification of rhesus macaque MHC class I amplicons (Table 3) were described previously (O'Leary et al., 2010) and were adapted to the requirements of the 454 GS Junior System. cDNA-PCR amplicons, spanning $367 \mathrm{bp}$ of MHC class I exon two and three were synthesized using the BioTherm. ${ }^{\mathrm{TM}}$ Taq-DNA polymerase. For each cDNA sample forward and reverse primers with identical MIDs were used.

PCR reaction mix:

$3 \mu 1 \quad$ Bio Therm. ${ }^{\mathrm{TM}} 10 \mathrm{x}$ Reaction Buffer

$0.2 \mu \mathrm{l} \quad$ dNTP-Mix (each $25 \mathrm{mM}$ )

$1 \mu \mathrm{l} \quad$ forward Primer $(10 \mu \mathrm{M})$

$1 \mu \mathrm{l} \quad$ reverse Primer $(10 \mu \mathrm{M})$

$0.2 \mu \mathrm{l} \quad$ BioTherm. ${ }^{\mathrm{TM}}$ Taq-DNA polymerase $(5 \mathrm{U} / \mu \mathrm{l})$

$23.6 \mu \mathrm{l} \quad \mathrm{HPLC}-\mathrm{H}_{2} \mathrm{O}$

$1 \mu \mathrm{l} \quad \mathrm{cDNA}$

$\underline{\text { PCR conditions: }}$

\section{Temperature Time}

$\left.\begin{array}{lll}\text { Initial denaturation } & 94^{\circ} \mathrm{C} & 3 \mathrm{~min} \\ \text { Denaturation } & 94^{\circ} \mathrm{C} & 15 \mathrm{sec} \\ \text { Annealing } & 60^{\circ} \mathrm{C} & 45 \mathrm{sec} \\ \text { Elongation } & 72^{\circ} \mathrm{C} & 1 \mathrm{~min}\end{array}\right]-35$ cycles

\subsubsection{Gel electrophoresis of DNA in agarose gels}

Gel electrophoresis was used to separate DNA fragments by size by applying an electric field to move the negatively charged molecules through an agarose matrix. DNA was visualized under UV light by using EtBr, a dye which intercalates with DNA.

To determine the size or for the purification of PCR products $1 \%$ horizontal agarose gels were used. The agarose was dissolved in $1 \mathrm{x}$ TBE buffer and boiled up. After cooling to $\sim 55^{\circ} \mathrm{C} \mathrm{EtBr}$ was added to a final concentration of $0.05 \mathrm{ng} / \mathrm{ml}$. The gel was casted in an electrophoresis camber and after polymerization it was covered with $1 \mathrm{x}$ TBE electrophoresis buffer. The DNA samples were mixed with DNA loading buffer and loaded into the gel. For 
the separation by size a constant voltage of $120 \mathrm{~V}$ was applied. DNA Ladder $100 \mathrm{bp}$ Plus was used for size determination.

\subsubsection{DNA extraction from agarose gels}

To purify PCR products from agarose gels, the DNA was visualized under UV light and cut out of the gel using a sterile scalpel. The extraction was performed using the QIAquick Gel Extraction Kit according to the supplier`s recommendations.

\subsubsection{Amplicon Library Preparation}

cDNA-PCR amplicons (2.2.4) were separated by size using $1 \%$ agarose gel in $1 \mathrm{x}$ TBE buffer and purified. To guarantee salt free samples for 454 sequencing, the cDNA-PCR amplicons were additionally purified by using Agencourt AMPure XP and the concentration was determined by fluorometry using the Quant-iT PicoGreen dsDNA Assay Kit according to protocol instructions (454 GS Junior, Roche). The sample concentration of each amplicon was calculated in molecules/ $\mu 1$ using the following equation.

$$
\text { Molecules } / \mu l=\frac{(\text { Sample conc. } ; n g / \mu l) \times 6.022 \cdot 10^{23}}{656.6 \times 10^{9} \times \text { amplicon length } ; b p}
$$

Amplicons were diluted with $1 \times$ TE buffer to a final concentration of $1 \times 10^{9}$ molecules $/ \mu 1$ and equal volume of 12 diluted amplicons were pooled to prepare one amplicon library. The purity of each library was confirmed by using a 2100 Bioanalyzer (Agilent). Libraries were finally diluted with $1 \times$ TE buffer to a concentration of $1 \times 10^{6}$ molecules/ $\mu 1$.

\subsubsection{Sequencing (GS Junior System, Roche)}

The emPCR, bead recovery and sequencing were performed according to the manufactures instructions (454 GS Junior, Roche). Each pool of twelve samples was sequenced on one PicoTiterPlate. The emPCR conditions for MHC class I amplicon libraries were performed following the standard protocol instructions. The emPCR conditions for KIR amplicon libraries were performed according to the recommendations for sequencing of long-length amplicon libraries ( $\geq 550 \mathrm{bp}$ ). The ratio of DNA and Capture Beads used for emPCR was in the order of 1 to 2 molecules per bead. 
emPCR reaction mix for standard and long-length amplicon libraries:

Volumes $(\mu \mathrm{l})$ for long-length amplicon libraries are shown in brackets.

$\begin{array}{ll}205 \mu \mathrm{l}(179 \mu \mathrm{l}) & \text { Mol. Bio. Grade Water } \\ 260 \mu \mathrm{l}(260 \mu \mathrm{l}) & \text { Additive } \\ 135 \mu \mathrm{l}(149 \mu \mathrm{l}) & 5 \mathrm{x} \text { Amplification Mix } \\ 40 \mu \mathrm{l}(52 \mu \mathrm{l}) & \text { Amplification Primer (A or B) } \\ 35 \mu \mathrm{l}(35 \mu \mathrm{l}) & \text { Enzyme Mix } \\ 1 \mu \mathrm{l}(1 \mu \mathrm{l}) & \text { PPiase }\end{array}$

emPCR conditions:

\section{Standard Library $(\leq \mathbf{5 5 0}$ bp)}

$1 \mathrm{x} \quad 4$ minutes at $94^{\circ} \mathrm{C}$

50x $\quad 30$ seconds at $94^{\circ} \mathrm{C}, 4.5$ minutes at $58^{\circ} \mathrm{C}, 30$ seconds at $68^{\circ} \mathrm{C}$

$1 \mathrm{x} \quad 10^{\circ} \mathrm{C}$ on hold

\section{Long-length amplicon library $(\geq 550 \mathrm{bp})$}

$1 \mathrm{x} \quad 4$ minutes at $94^{\circ} \mathrm{C}$

$50 \mathrm{x} \quad 30$ seconds at $94^{\circ} \mathrm{C}, 10$ minutes at $60^{\circ} \mathrm{C}$

$1 \mathrm{x} \quad 10^{\circ} \mathrm{C}$ on hold

\subsubsection{Bioinformatic analysis of $\mathbf{4 5 4}$ Sequencing data (KIR)}

After the initial quality assessment using standard settings of the 454 software, sequencing raw data were analysed by custom Perl scripts. Separate library files for each individual were generated by sorting sequencing reads with identical multiplex identifiers (MID). The MID sequences, comprising $10 \mathrm{nt}$ each, and the target-specific primer sequence (20 nt) were cut off at the 5 end if both are perfectly matching. The design of KIR amplicon primers is illustrated in detail in 2.2.4. Sequencing reads with a minimum of $370 \mathrm{nt}$ were filtered and longer reads are cut to the same length $(370 \mathrm{nt})$ at the $3^{\prime} \mathrm{end}$, with respect to an average read length of $400 \mathrm{nt}$ (as guaranteed by the 454 sequencer). Each library file was then compressed by including different sequences only and encoding the sequence frequencies into FASTA IDs:

$><$ animal ID $>-<$ number $>\#<$ frequency $>$.

Reads with a frequency of $\geq 5$ were filtered in order to reduce sequencing errors. The filtered reads were aligned against reference sequences of all known rhesus macaque KIR sequences (IPD KIR database, macaque sequence database obtained from James Robinson, Anthony Nolan Trust, UK, and Libby Guethlein, Stanford University, USA) using BLAST. Three 
alignment profiles were derived for each individual, one based on perfectly matching reads only, one allowing a maximum of two mismatches and one allowing a maximum of six mismatches compared to the reference sequences, respectively. As forward and reverse reads were aligned separately, sequences with a maximum of four or 12 mismatches, respectively, over the full read length were included in the final preparation of KIR transcription profiles of each rhesus macaque. Since the sequenced KIR region of around $560 \mathrm{nt}$ (623 nt with primer regions) is not fully covered by one read, sufficient counts $(\geq 5)$ of matching forward and reverse reads needed to be found. The overlap between forward and reverse reads covers around one third (180 nt) of the KIR region.

The alignment profiles allowing a maximum of two mismatches compared to the reference sequences were used for the final preparation of $K I R$ transcript profiles. Frequencies of filtered forward and reverse reads were summed up and the relative contribution of each KIR transcript was compared to the total number of KIR transcripts identified in each individual. KIR sequences were only considered to be present in an animal if they represent at least $0.9 \%$ of total KIR sequencing reads from that animal in order to reduce errors introduced by PCR artefacts. This threshold was chosen as the lowest number of sequencing reads, which was thereby considered to represent a KIR transcript, still comprised 20 even perfectly matching sequence reads. Ambiguous reads, matching more than one KIR gene, were excluded if a clear division with the perfect alignment profiles was not possible, too. If forward or reverse reads for a KIR transcript were not detected within an individual, the detected sequence reads were only taken into account if the missing reads were unique in the alignment profile allowing six mismatches. The comprehensive data analysis of 454 sequencing data for KIR transcripts is additionally described in chapter 3.1 .

\subsubsection{Bioinformatic analysis of 454 Sequencing data (MHC class I)}

After the initial quality assessment using standard settings of the 454 software, sequencing raw data were analysed by custom Perl scripts. Separate library files for each individual were generated by sorting sequencing reads with identical MIDs. The MID sequences and targetspecific primer sequence were cut off at the 5 end if both are perfectly matching. Sequencing reads with a minimum of $350 \mathrm{nt}$ were filtered and longer reads are cut to the same length (350 nt) at the 3'end. Each library file was then compressed as described in Section 2.2.9.

Reads with a frequency of $\geq 5$ were filtered in order to reduce sequencing errors. The filtered reads were aligned against reference sequences of all known rhesus macaque MHC class I 
sequences (http://www.ebi.ac.uk/ipd/mhc/nhp/index.html; Dec. 2011) using BLAST. One alignment profile was derived for each individual, based on perfect matches of these reads only. In a second profile reads with $\leq 2$ copies and $\leq 2$ mismatches compared to the reference database were included. Since these reads most likely result from sequencing errors and lowfrequent reads typically comprise a large fraction of all reads of a run, their counts may be safely used to increase the numbers of perfect matches from the first profile.

Note that the sequenced MHC class I region of around $367 \mathrm{bp}$ is already fully covered by a single (forward or reverse) read. Frequencies of filtered forward and reverse reads were summed up and the relative contribution of each MHC class I transcript was compared to the total number of transcripts identified in each individual. Ambiguous reads were an exception and if reads were identical for more than one MHC class $I$ allele, the result is shown as a set of alleles (e.g Mamu-B*050/078). MHC class I sequences were only considered to be present in an animal if they represent at least $1 \%$ of total MHC class I sequencing reads from that animal in order to reduce errors introduced by PCR artefacts.

\subsubsection{Statistical analysis}

\section{$\underline{\text { Statistical significance }}$}

Statistical differences between (the means of) two independent populations were calculated performing two-tailed unpaired Student's t-tests or Fisher`s exact test. $P$-values of $\leq 0.05$ were considered statistically significant. To test for differences between paired observations, like $K I R$ transcription levels of a group of individuals over the course of SIV disease (three time points), two-tailed paired t-tests were performed.

\section{Correlation and linear regression}

The Pearson correlation coefficient $r$ ranges from -1 to +1 and quantifies the direction and magnitude of correlation between two continuous variables $X$ and $Y$. A positive correlation $(r>0)$ indicates that both variables increase or decrease. A negative correlation $(r<0)$ indicates that one variable increases while the other decreases. Two-tailed $P$-values were used to test the statistical significance of the correlation, i.e., if $r$ is significantly different from zero.

Linear regression analyses whether there is a linear relationship between two variables $X$ and $Y$ by fitting a strait line through all $n$ measured data points $(x, y)$ such that their squared vertical distances to the line are minimized. The slope of the fitted line reflects the correlation of $X$ and $Y$. The corresponding $P$-values show whether the slope is significantly different from 
zero. A $95 \%$ confidence interval is defined by the two curves surrounding the best-fit line. There is a $95 \%$ chance that this area contains the true regression line.

\subsubsection{Quantitative real-time PCR gene expression with the Biomark TM System (Fluidigm)}

Quantitative real-time PCR was performed by using a microfluidic-based real-time PCR approach (Biomark Fluidigm) with 48.48 dynamic array chips. This system enables to analyse 2304 reactions within on run and the small number of pipetting steps allows high accuracy. The IFC Controller and the Biomark ${ }^{\mathrm{TM}}$ Real-Time PCR System are manufactured by Fluidigm.

DELTAgene ${ }^{\mathrm{TM}}$ Assay is a custom based qPCR gene expression assay by Fluidigm was used. It was delivered as a forward and reverse primer mix with each primer at a concentration of $100 \mu \mathrm{M}$. The array contains 48 forward and reverse primer mix, amplifying 48 specific genes (listed in Table 4). Equal volumes of each primer mix were pooled (STA Primer Mix) and diluted to a final concentration of $500 \mathrm{nM}$ with1 x DNA Suspension Buffer.

The manufacturer protocol (SsoFast EvaGreen SuperMix with Low ROX with the 48.48 Dynamic Array IFCs) was used to perform gene expression analyses on the BioMark ${ }^{\mathrm{TM}}$ System, with the following exceptions and options.

The STA (specific target amplification) pre-amplification PCR was performed using $1.25 \mu 1$ cDNA in addition to $2.5 \mu 1$ TaqMan PreAmp Master Mix, $0.5 \mu 1$ STA Primer Mix and filled up to a volume of $5 \mu \mathrm{l}$ with 1 x DNA Suspension Buffer. For each 48.48 gene expression run three negative controls (1 x DNA Suspension Buffer) were analysed. The STA reactions were performed by using the following thermal cycle conditions.

$1 \mathrm{x} \quad 10$ minutes at $95^{\circ} \mathrm{C}$

12x $\quad 15$ seconds at $95^{\circ} \mathrm{C}, 4$ minutes at $60^{\circ} \mathrm{C}$

$1 \mathrm{x} \quad 4^{\circ} \mathrm{C}$ on hold

Each PCR reaction was treated with exonuclease I according to the manufactures instructions to remove unincorporated primers. After the incubation step each reaction mix was diluted 1:5 with $1 \times$ DNA Suspension Buffer.

The sample pre-mix, containing the STA and Exo I-treated samples, 2 x SsoFast EvaGreen Supermix with Low ROX, and $20 \mathrm{x}$ DNA Binding Dye Sample Loading Reagent, were prepared according to the manufactures instructions. The $10 \mathrm{x}$ Assay mix contains $2 \mathrm{x}$ Assay 
Loading Reagent, 1 x DNA Suspension Buffer, and Assay Mix Primer Pairs (100 $\mu$ M) and was prepared according to the manufactures instructions.

For the priming of the 48.48 Dynamic Array IFC chip and the loading of sample and assay mix, the IFC Controller was used. The IFC Controller utilizes pressure to control the valves in the chips and load samples and gene expression assay reagents into the reaction chambers.

After the loading the chip run was performed on the Biomark ${ }^{\mathrm{TM}}$ system, which is a real-time PCR instrument designed to thermal cycle these microfluidic chips and image the data in real time. The data collection software was programmed following the protocol instructions.

Initial denaturation $\quad 95^{\circ} \mathrm{C} 60 \mathrm{sec}$

Denaturation $\quad 96^{\circ} \mathrm{C} 5 \mathrm{sec}$

Annealing/Extension $\left.\quad 60^{\circ} \mathrm{C} 20 \mathrm{sec}\right\} 35$ cycles

Melt Curve use default

\subsubsection{Determination of relative gene expression}

GAPDH was used as the reference gene and the values were used for normalisation to determine the delta $\mathrm{C}_{\mathrm{T}}\left(\Delta \mathrm{C}_{\mathrm{T}}\right)$ values. The $\Delta \mathrm{C}_{\mathrm{T}}$ values were obtained directly from the data collection software (Fluidigm). Samples obtained prior to infection with SIV (pre infection) were used as the reference sample for each gene and individual to calculate $\Delta \Delta C_{T}$ values. Relative gene expression values were determined using the $2^{-\Delta \Delta \mathrm{CT}}$ method of Livak and Schmittgen (2001). All data from the 48.48 dynamic array chips were reported as fold expression relative to the pre infection (SIV) sample of each individual and analysed gene. 


\section{Results}

In this study, retrospective analyses of PBMC samples of a cohort of 52 SIV-infected rhesus macaques (SIVmac239 and SIVmac251) were performed. In order to identify "advantageous" and "disadvantageous" rhesus macaque KIR and MHC class I transcripts, which are associated with different disease progression during experimental SIV infection, the cohort was divided into two groups according to plasma viral loads at set point. 28 animals showed high viral load (HVL > 10 6 viral copies/ $\mathrm{ml}$ of plasma) and a cohort of 24 animals showed low viral load at set point (LVL $<10^{5}$ viral copies/ $\mathrm{ml}$ of plasma) (Figure 5). All PBMC samples were provided by Professor Aftab Ansari (Department of Pathology, Emory University School of Medicine, Atlanta) and the individuals are listed in 2.1.1.

This study aimed at identifying KIR genes that are associated with SIV disease pathogenesis. Transcribed KIR genes within individuals were of particular interest. The sequence-specific PCR (SSP) KIR typing method was developed by our group (Kruse et al., 2010) and can be used to check for the presence or absence of an individual gene or even allele. However, the next generation sequencing technology (Roche/454 Titanium) enables sequencing of cDNAPCR amplicons and has crucial advantages compared to other typing strategies.

In this study, the recently published KIR typing method (Moreland et al., 2011) was adapted on the 454 GS Junior system (Roche) to characterise the SIV-infected rhesus macaque cohort and to identify disease-associated KIR transcripts. This sequence-based typing approach has a high sensitivity and throughput and enables to analyse KIR transcripts within one individual in a very comprehensive way. Multi-identifier (MID) tagged oligonucleotides amplify a region of $623 \mathrm{bp}$ spanning the majority of D1, all of D2, and part of the stem region of KIR transcripts. Primer binding sites are highly conserved in all published rhesus macaque KIR3D sequences, and in almost all published KIR1D sequences. KIR2DL04 lacks the D1 region and therefore the 5 primer-binding site will not be amplified by this approach (see Figure 3). A disadvantage is the partly missing discrimination between alleles, which is due to the limited length of $623 \mathrm{bp}$ amplicons and the sequence homology of about 90-95\% of KIR genes. Therefore, the KIR typing results were confined to the gene level, and were not studied on the allele level in this study.

Moreover, this approach provides estimates of the relative level of each KIR gene transcription. KIR transcription profiles, characterising the transcribed $K I R$ genes detected within each individual along with the relative expression level of each $K I R$, were established for individuals for different time points over the course of the SIV disease (pre, acute, chronic 
phase of infection). Thereby, the influence of infectious SIV disease with regard to differences in the KIR transcript repertoire, and particularly on the relative level of $K I R$ gene transcription within each individual, was investigated. This comparison of $K I R$ transcription profiles is possible because of the high reproducibility of the published approach. Moreland et al. (2011) compared sequencing data of uninfected rhesus macaques between two independent sample preparations and PCR replicates, demonstrating that the relative expression levels of $K I R$ transcripts are highly reproducible, at least for those cases where the average transcription of a KIR gene was greater than $3 \%$ of total reads. Only KIR transcripts, which were present in less than $3 \%$ of total reads, were missed in some reaction, as the variability is greater in transcripts that are expressed at lower levels. The high reproducibility of the relative expression levels of KIR transcripts was also confirmed in this study (3.4.1).

Taken together two different "KIR profiles" were analysed in this study: The KIR transcript repertoire, indicating the content of transcribed $K I R$ genes within an individual, independent of the relative expression level (Figure 7A), and the KIR transcription profile, indicating transcribed KIRs along with their relative expression levels (Figure 7B). An illustration of the experimental set-up is shown in Figure 7.

Additionally, pre infection samples were used to characterise MHC class I transcripts in the same macaque cohort, by performing 454 sequencing of cDNA-PCR amplicons (O`Leary et al., 2009). 


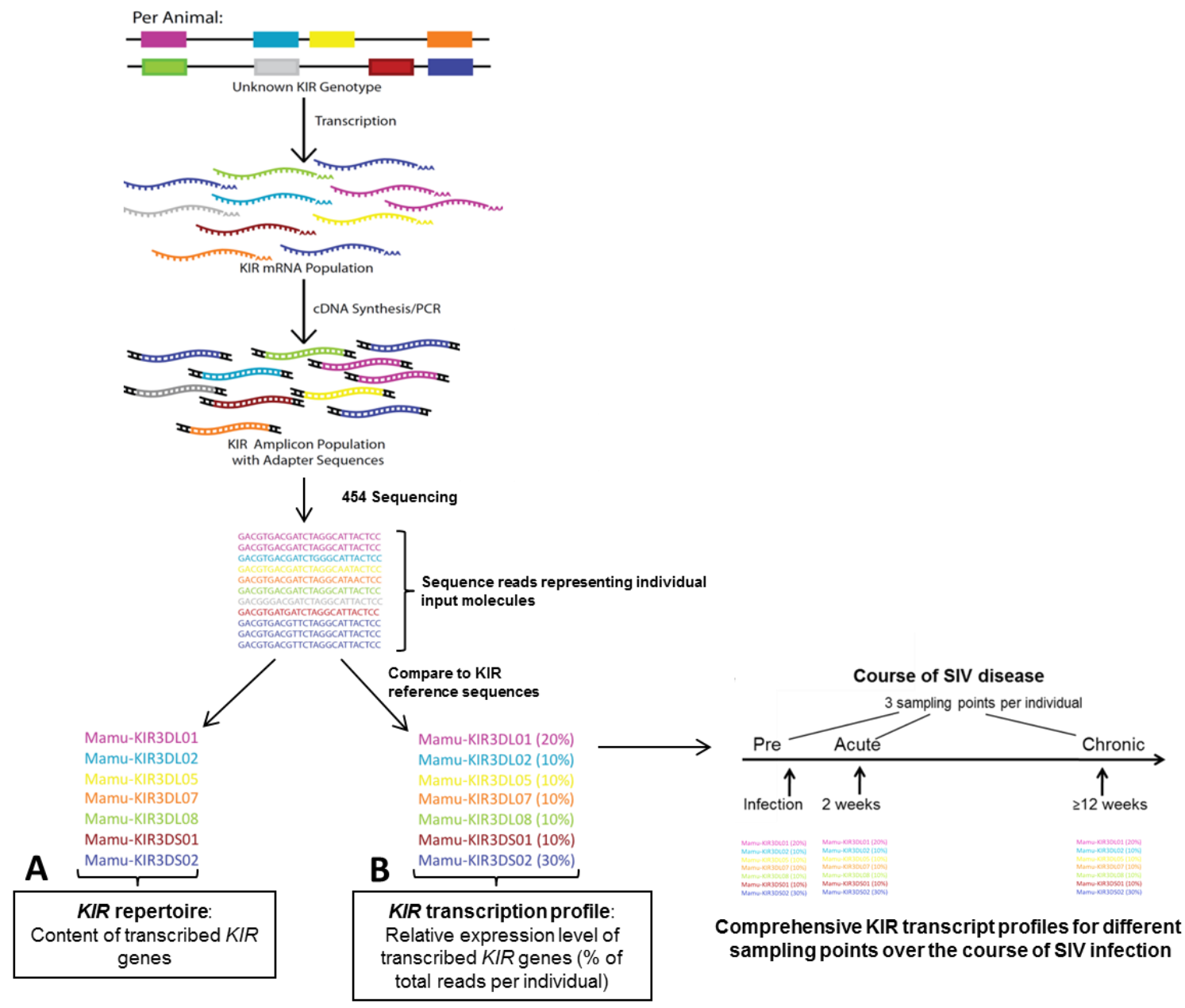

Figure 7 Experimental set-up for comprehensive KIR typing using 454 sequencing.

Total RNA was isolated from PBMCs obtained at three different time points of SIV infection (pre, acute, chronic phase of infection). PCR was performed using KIR-specific primers with adaptor tags according to Moreland et al. (2011) and cDNA-PCR amplicons represent all KIRs transcribed by the rhesus macaque. Amplicon pools of up to twelve samples were sequenced within one run and sequence reads represent individual input molecules, which were compared to KIR reference sequences. The analysis provides information on (A) the transcribed KIR gene content (B) along with the relative transcription level of KIR genes within each animal. KIR transcription profiles were compared for the different time points over the course of the SIV disease. (Figure modified from Moreland et al., 2011)

\subsection{Best fitting approach to analyse 454 sequencing data for $K I R$ transcripts}

The most striking issue of the 454 Sequencing System is the read length expectation of about $500 \mathrm{bp}$ utilized with the chemistry of the GS Junior System. However, the length of amplicons used in this study is above the expected read length. The amplicons comprise $623 \mathrm{bp}$ target sequence in addition to $30 \mathrm{bp}$ flanking MID and primer sequences and are not sequenced in their full length in individual reads. Therefore, bidirectional reads (forward and 
reverse reads) were analysed separately and the number of reads was added. To reliably cover the entire sequence of an amplicon, a sufficient overlap of at least $180 \mathrm{bp}$ was achieved in the middle of the amplicon sequence between the reads in both directions. Because multiple individuals were sequenced within a single run, complete amplicon sequences of each individual were concatenated by using forward and reverse primers containing an identical MID. The pre-processing of raw data and the filter strategies are described in chapter 2.2.9.

Three different approaches were tested to establish the best applicable strategy to analyse the 454 sequencing data of KIR cDNA-PCR amplicons. The results of all approaches were compared to finally exclude an influence on the results obtained in this study.

\section{Approaches:}

1. Sequencing reads have to match the known reference sequences (IPD KIR database, $2^{\text {nd }}$ April 2011) at $100 \%$ (perfect matches)

2. Sequencing reads have a maximum of two mismatches compared to reference sequences (in total four mismatches of forward and reverse reads)

3. Sequencing reads have a maximum of six mismatches compared to reference sequences (in total 12 mismatches of forward and reverse reads)

Approach 1 uses sequencing reads that perfectly match the known KIR reference sequences and allows to distinguish between all known $K I R$ genes, but still failed to distinguish between all known KIR alleles, due to the limited length of the sequenced amplicon. In human 861 KIR alleles are currently published (IPD KIR database, $2^{\text {nd }}$ April 2011), but with only 175 known sequences in rhesus macaques more than threefold less $K I R$ alleles are described. However, rhesus macaque KIR haplotypes are as polymorphic as human KIR haplotypes, and were recently shown to exhibit even higher variability as human KIR haplotypes (Kruse et al., 2010; Blokhuis et al., 2010; Blokhuis et al., 2011), implying that many KIR alleles are not identified. The assumption that a large number of rhesus macaque $K I R$ alleles are still unknown is the most considerable disadvantage aspect of approach 1, as sequencing reads with one single nucleotide polymorphism compared to the reference sequences are excluded. In contrast, the use of sequencing reads with a large number of mismatches compared to the reference sequences of approach 3 complicates the distinction between the different sequencing reads whether they belong to distinct alleles of the same KIR gene or to a distinct $K I R$ gene. The sequence homology of about $90-95 \%$ of KIR genes contributes to ambiguous sequencing reads, matching for more than one KIR gene. The data analysis with approach 3 was challenging and in many cases ambiguous. 
Approach 2 has the advantage to identify reads that differ in four nucleotides at most to the reference sequences. For example, individual mm48 is KIR3DL10 negative, if analysed with approach 1, but the analysis with approach 2 revealed 100 unique KIR3DL1O reads (44 forward and 56 reverse reads), comprising even $12.18 \%$ of total reads within that animal. All 44 forward and 56 reverse reads are identical and sequences from both directions have only one SNP each compared to the reference sequence. Moreover, the presence of KIR3DL10 transcripts in individual mm48 was confirmed by two additional samples of that individual. The identical SNPs within the analysed KIR3DL1O sequence were also identified in individual mm46. Similarly, three individuals were KIR3DLO7 negative, if analysed with approach 1, but the analysis with approach 2 identified unique KIR3DL07 forward and reverse reads, comprising 2.4-13.23\% of total reads within the particular animal. The presence of KIR3DL07 transcripts was also confirmed by two additional samples of these individuals. Another disadvantage of approach 1 is that sometimes only forward or reverse reads of specific KIR genes were detected, due to a single mismatch compared to the reference sequences. The analysis of e.g. animal mm5 revealed 140 perfect reverse reads matching KIR3DS05, but failed to identify any forward read. With approach 2, the same 140 reverse reads were identified and additionally 278 unique forward reads matching KIR3DSO5. This results in an increase of the relative expression level of KIR3DSO5 transcripts of $11.95 \%$ of total reads in individual mm5. Thereby, the relative expression levels of the remaining KIR transcripts identified within mm5 are changed, but not significantly (Table 5).

Table 5 Comparison of the relative expression levels of KIRs of individual mm5 established with approach 1 and 2

\begin{tabular}{cccccc}
\hline \hline Approach & KIR1D & KIR3DL01 & KIR3DL10 & KIR3DL11 & KIR3DS05 \\
\hline 1 & $7,36 \%$ & $10,60 \%$ & $8,63 \%$ & $65,71 \%$ & $7,69 \%$ \\
2 & $6,30 \%$ & $9,07 \%$ & $7,38 \%$ & $57,61 \%$ & $19,64 \%$ \\
\hline
\end{tabular}

Approach 2 also rarely failed to cover the complete amplicon sequence of $K I R$ transcripts, similarly to approach 1 . If forward or reverse reads of a specific KIR transcript failed to be detected with approach 2 , the detected sequencing reads were only considered, if the missing part could be covered with unique reads by approach 3 . It is necessary to recheck the data in order to reduce errors introduced by PCR artefacts. If unique reads were identified with approach 3, only the number of reads detected with approach 2 was considered. For example, the analysis of individual mm14 failed to detect forward reads of KIRID if analysed with approach 2, but 13 unique sequencing reads could be identified with approach 3 . However, including only forward or reverse reads detected with approach 2 , or adding the missing 
sequencing reads identified with approach 3, has no striking influence on the relative expression levels of all KIR transcripts identified in this individual, as shown in Table 6.

Table 6 Comparison of two different modifications of approach 2 (individual mm14)

\begin{tabular}{|c|c|c|c|c|c|c|c|c|}
\hline \multirow[b]{2}{*}{ KIR } & \multicolumn{4}{|c|}{ Approach 2} & \multicolumn{4}{|c|}{ Approach $2+$ missing reads Approach 3} \\
\hline & \# Fwd $^{\mathrm{a}}$ & \# $\operatorname{Rev}^{\mathbf{b}}$ & SUM $^{c}$ & $\% T^{d}{ }^{d}$ & \# Fwd $^{\mathrm{a}}$ & \# Rev ${ }^{\mathbf{b}}$ & SUM $^{c}$ & $\% \operatorname{TR}^{d}$ \\
\hline 1D & nd & 46 & 46 & $4,62 \%$ & 13 & 46 & 59 & $5,85 \%$ \\
\hline 3DL01 & 60 & 186 & 246 & $24,70 \%$ & 60 & 186 & 246 & $24,38 \%$ \\
\hline 3DL05 & 7 & 45 & 52 & $5,22 \%$ & 7 & 45 & 52 & $5,15 \%$ \\
\hline 3DL10 & 38 & 109 & 147 & $14,76 \%$ & 38 & 109 & 147 & $14,57 \%$ \\
\hline 3DS02 & 27 & 175 & 202 & $20,28 \%$ & 27 & 175 & 202 & $20,02 \%$ \\
\hline 3DS05 & 87 & 216 & 303 & $30,42 \%$ & 87 & 216 & 303 & $30,03 \%$ \\
\hline
\end{tabular}

${ }^{a}$ Total number of sequencing reads matching corresponding KIR reference sequences (forward)

${ }^{\mathrm{b}}$ Total number of sequencing reads matching corresponding KIR reference sequences (reverse)

${ }^{\mathrm{c}}$ Sum of forward and reverse reads

${ }^{\mathrm{d}}$ Percentage of total reads, relative contribution of the indicated KIR transcript to the total of transcripts identified within the animal

nd $=$ not detected

Within a single animal (mm44) only, the detected reverse reads for KIR3DSO7 and KIR3DSW08 were excluded, as the missing forward reads are not unique for one of these KIR sequences, if rechecked with approach 3.

The incident that approach 2 failed to detect forward or reverse sequencing reads occurred rarely, and the influence of missing reads is negligible for the comparison of the relative expression levels of $K I R$ transcripts within a single animal over the course of SIV infection. Missing reads mainly occurred in KIR transcripts that are expressed at very low levels, or resulted from more than two polymorphisms in the analysed sequence. The analyses of differences in $K I R$ gene expression within the total cohort can be influenced by missing sequencing reads of single $K I R$ transcripts, but like already mentioned, missing reads occurred infrequent and mainly in lower expressed KIR genes, and were tested to have no critical impact on the results obtained in this study (data not shown).

To finally exclude an influence of the analytical approach on the results obtained in this study, the relative expression levels of KIR transcripts identified in the rhesus macaque cohort were analysed with the approaches 1 and 2, and the results were compared (Figure 8). The graph shows the results obtained with approach 1 (black) and 2 (red) and clearly indicates that the relative expression levels of each KIR transcript are not significantly different ( $p$-value $>0.05$ ) between both analysis approaches. Thus, neither the significant differences of KIR transcript frequencies observed between the HVL and LVL cohort, nor the differences of the relative transcription levels of KIR genes between both cohorts are critically influenced by the 
analytical approach. The changes of KIR transcription levels over the course of SIV disease are also unaffected by the used approach (data not shown). Approach 3 was excluded, as the high number of approved mismatches in combination with the generally high sequence identity of KIR genes often results in ambiguous reads and challenges the distinct allocation of reads.

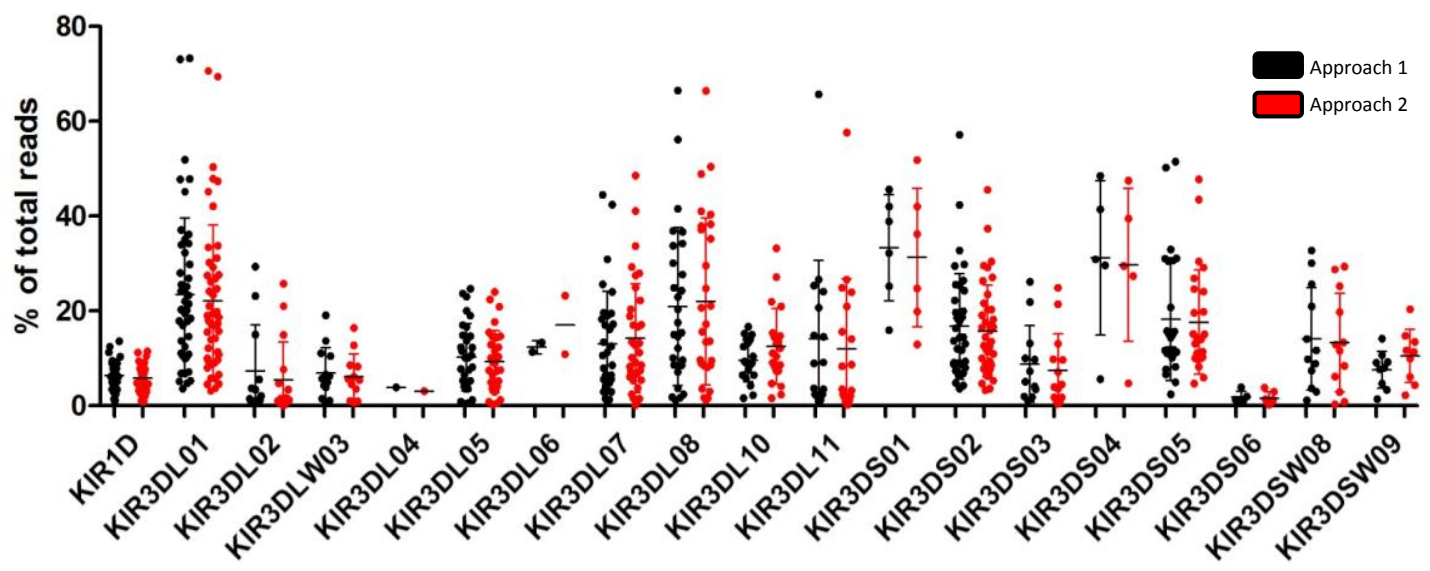

Figure 8 Relative expression levels of $K I R$ transcripts in the macaque cohort analysed with approach 1 and 2.

The y-axis indicates the percentage of total sequencing reads per individual for each KIR transcript. All KIR transcripts identified in the rhesus macaque cohort $(\mathrm{n}=51$; for individual $\mathrm{mm} 24$ no pre infection sample was available) are shown. Averages and standard derivation (SD) are represented by bars. Each dot indicates the relative expression level of the KIR transcript in a single individual. Only samples prior to infection with SIV were compared.

Taken together, the extensive comparisons of the results generated with different approaches ensure that on the one hand the enormous amount of sequencing data can be actually handled, and on the other hand that there is no (or only minimal) influence of the applied analysis strategy on the results obtained in this study. Nevertheless, all these findings showed that the analysis of the 454 sequencing data with approach 2 is the best fitting strategy to characterise the rhesus macaque cohort for KIR transcripts in a comprehensive way. Sequencing reads with only a few polymorphisms $(\leq 4)$ compared to the reference sequences are included and an intergenic discrimination is still possible, with very few exceptions.

The analysis of MHC class I sequences was less complex, due to the shorter fragment size of cDNA-PCR amplicons (423 bp). Sequencing reads usually cover the complete target sequence and missing forward or reverse reads were an exception. In addition, the database (http://www.ebi.ac.uk/ipd/mhc/nhp/index.html) of MHC class I sequences for rhesus macaques is much more comprehensive than the KIR database and it is much easier to assign 454 sequencing reads to distinct $M H C$ class $I$ sequences of rhesus macaques as the individual 
MHC class I genes differ among each other much more compared to KIR genes. The preprocessing and the used approach of final data analysis for $M H C$ class I sequencing data are described in chapter 2.2.10.

\subsection{Characterisation of $K I R$ and MHC class I genes of the rhesus macaque cohort}

The cohort of $52 \mathrm{SIV}$-infected rhesus macaques was characterised for KIR and MHC class I transcripts by performing retrospective analyses of PBMC samples, which were obtained prior to infection with SIV (pre infection). Comprehensive KIR and MHC class I transcript profiles of each rhesus macaque were established, respectively, by performing 454 sequencing of cDNA-PCR amplicons. For one individual (mm24) only a post infection sample (acute phase of infection) was included, as a pre infection sample was not available. However, individual $\mathrm{mm} 24$ was considered in some analyses, as the results obtained in this study have shown that the repertoire of transcribed KIR genes (content of $K I R$ transcripts, independent of the relative expression level of KIRs) within an individual, does not change over the course of SIV infection (see 3.4.1 and 3.4.4). As the relative transcription levels of KIR genes showed differences over the course of SIV infection (see 3.3.2 and 3.4) individual $\mathrm{mm} 24$ was excluded in these analyses. The pre-processing, filter strategies, and final analysis of 454 sequencing data are described in chapter 2.2.9 (KIR) and 2.2.10 (MHC class I).

\subsubsection{Frequency of distinct $K I R$ transcripts in the rhesus macaque cohort}

In the analysed cohort of rhesus macaques, 19 distinct $K I R$ gene transcripts were identified. Eleven $K I R$ transcripts belong to inhibitory $K I R(K I R I D, K I R 3 D L)$ and eight to activating $(K I R 3 D S)$ (Figure 9). While no KIR transcript could be identified in all animals, KIR3DLO1 was present in about $94 \%$ of the cohort. A similar frequency of KIR3DL01 transcripts ( 84 \%) was observed by Moreland et al. (2011) and confirms the assumption that KIR3DL01 acts as a framework gene in most rhesus macaque KIR haplotypes. Four additional inhibitory KIR transcripts, KIRID, KIR3DL05, KIR3DL07, and KIR3DL08, and two activating KIR transcripts, KIR3DSO2 and KIR3DSO5, showed high frequencies of $\geq 50 \%$ in the rhesus macaque cohort. Transcripts of KIR3DL04, KIR3DL06, KIR3DS01, KIR3DSO4 and KIR3DSO6 were found in a minor fraction $(\leq 12 \%)$ of the rhesus macaque cohort. The respective frequencies of all expressed KIR genes are shown in Figure 8 and Table 7. 


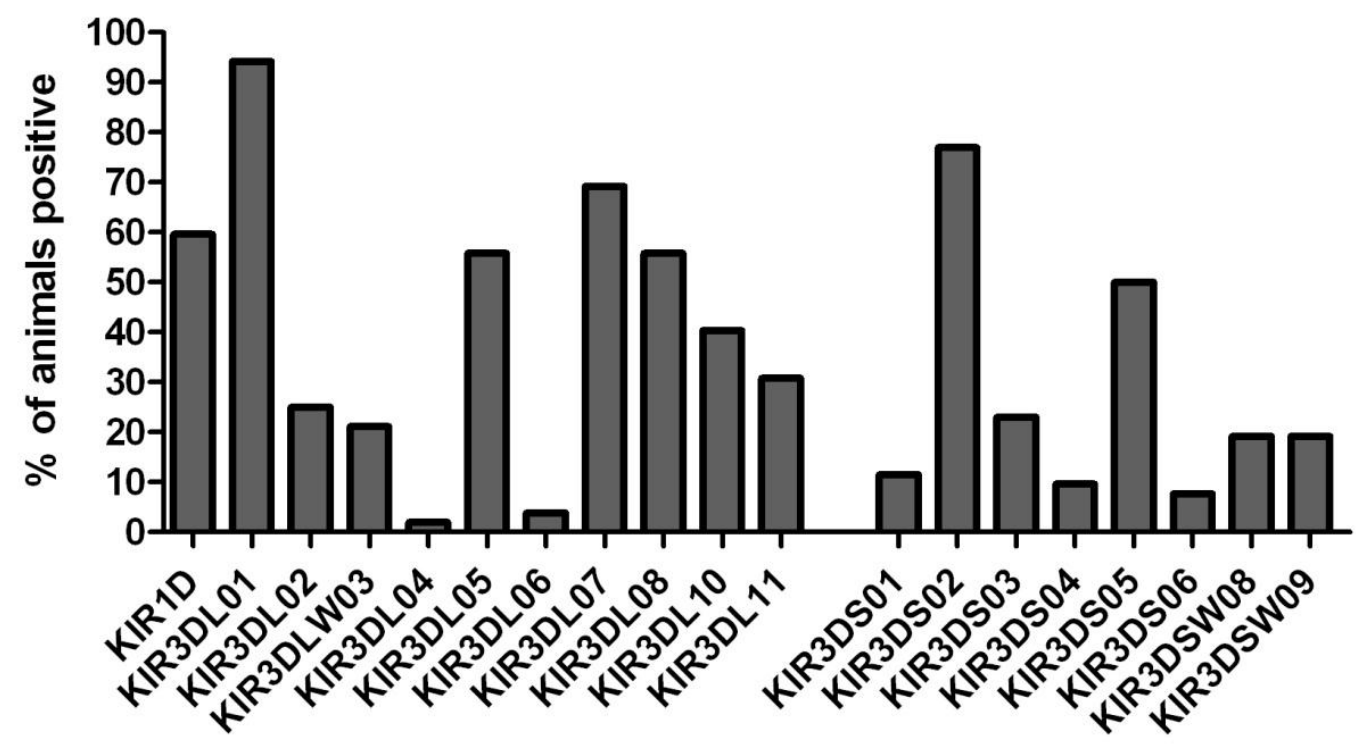

Figure 9 Identified KIR transcripts in the rhesus macaque cohort (pre infection).

The $\mathrm{y}$-axis indicates the percentage of animals within the cohort $(\mathrm{n}=52)$ that express the indicated $K I R$ transcript. Only pre infection samples were included (except for individual mm24, acute infection sample). KIR transcripts representing at least $0.9 \%$ of total reads within an individual were included in order to reduce errors introduced by PCR artefacts. KIR transcripts which are not listed here could not be identified in any animal within the cohort. KIR2DL04 is not amplified by the sequencing approach of cDNA-PCR amplicons and, therefore, not included.

Table 7 Frequencies of transcribed KIRs in the rhesus macaque cohort (pre infection*;

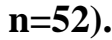

\begin{tabular}{llllll}
\hline \hline KIR & $\mathbf{n}^{\mathbf{a}}$ & $\mathbf{\%}^{\mathbf{b}}$ & KIR & $\mathbf{n}^{\mathbf{a}}$ & $\mathbf{\%}^{\mathbf{b}}$ \\
\hline 1D & 31 & 59.6 & 3DS01 & 6 & 11.5 \\
3DL01 & 49 & 94.2 & 3DS02 & 40 & 76.9 \\
3DL02 & 13 & 25.0 & 3DS03 & 12 & 23.1 \\
3DLW03 & 11 & 21.2 & 3DS04 & 5 & 9.6 \\
3DL04 & 1 & 1.9 & 3DS05 & 26 & 50.0 \\
3DL05 & 29 & 55.8 & 3DS06 & 4 & 7.7 \\
3DL06 & 2 & 3.9 & 3DSW08 & 10 & 19.2 \\
3DL07 & 36 & 69.2 & 3DSW09 & 10 & 19.2 \\
3DL08 & 29 & 55.8 & & & \\
3DL10 & 21 & 40.4 & & & \\
3DL11 & 16 & 30.8 & & & \\
\hline
\end{tabular}

* The acute infection sample of individual mm24 was included

a Total number of animals positive for the indicated KIR transcript

${ }^{\mathrm{b}}$ Percentage of animals positive for the indicated KIR transcript 


\subsubsection{Interindividual variation in expression levels of $K I R$ transcripts}

While SSP-KIR typing methods only provide information about presence and absence of $K I R$ genes per individual, a major advantage of this sequencing approach is that it also provides estimates of the relative expression level of each $K I R$ gene, and has the potential to detect new $K I R$ sequences. The relative contribution of each KIR transcript was compared to the total of $K I R$ transcripts identified in each individual (\% of total reads).

The comparison of the relative expression levels of all KIR transcripts identified showed striking differences (Figure 10). Some KIR transcripts, e.g. KIRID, KIR3DLW03, and KIR3DSW09, are consistently expressed at low levels of less than $20 \%$ of total reads within individuals. In contrast other KIR transcripts are expressed at higher levels such as KIR3DLO1 or KIR3DLO8, which can comprise approximately $70 \%$ of total sequencing reads within an individual, but were also documented to comprise less than $10 \%$ of total reads (Figure 10).

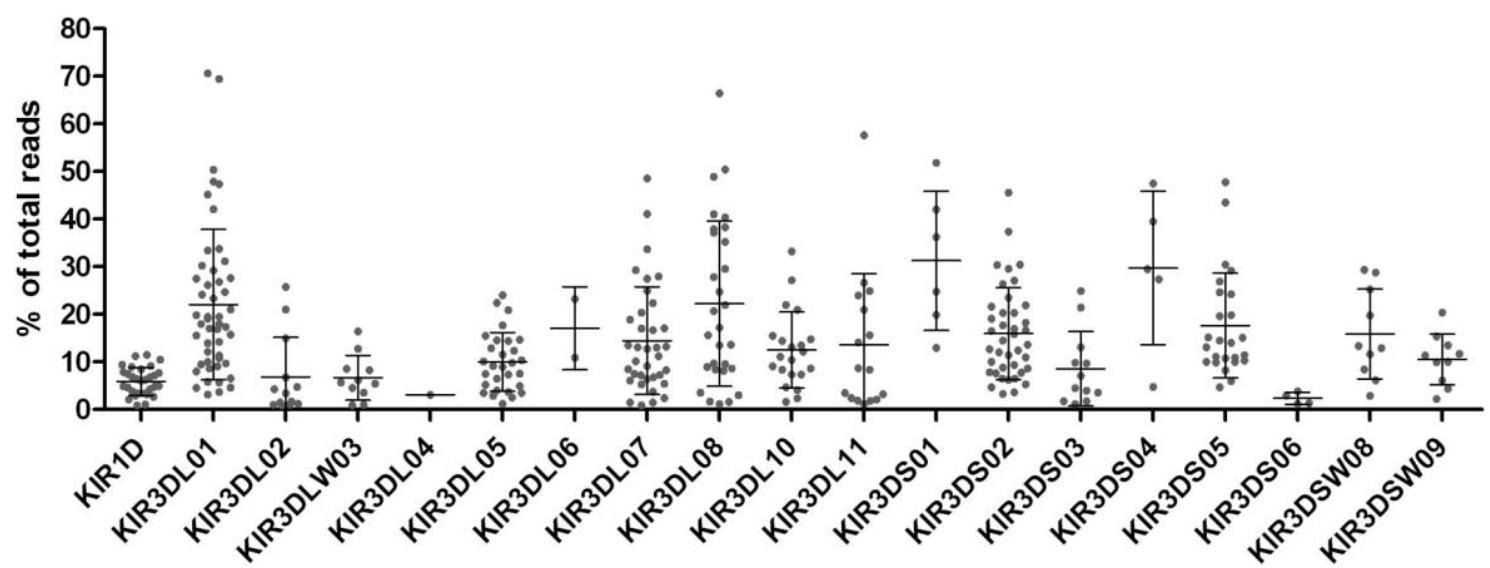

Figure 10 Relative contribution of KIR transcripts in the rhesus macaque cohort (pre infection). The $y$-axis indicates the percentage of total sequencing reads per individual for each $K I R$ transcript. Averages and SD are represented by bars. Dots represent the relative expression level of each KIR transcript to the total of KIR transcripts identified in each animal prior to infection with SIV (the acute infection sample of individual $\mathrm{mm} 24$ was excluded). KIR transcripts representing at least $0.9 \%$ of total reads were included to reduce errors introduced by PCR artefacts.

\subsubsection{Investigation of interindividual differences of $K I R$ transcripts}

The total number of individual KIR transcripts identified in each animal of the rhesus macaque cohort showed considerable differences. On average 2254 sequence reads were detected per individual. Animals with at least four and up to eleven different transcribed KIR genes were identified, while the majority of animals possessed six to seven distinct KIR transcripts. In each individual at least two inhibitory and one activating $K I R$ transcripts were documented, respectively. On average four to five distinct inhibitory KIR transcripts along 
with two distinct activating $K I R$ transcripts were detected in the cohort. While no animal possessed more activating than inhibitory KIR transcripts, four individuals with an equal ratio of both were identified. The average I:A ratio between inhibitory (I) and activating (A) KIR transcripts was calculated to be in the range of 1 to 5 and resulted in an average of 2.5. This I:A ratio implies that for every five inhibitory $K I R$ transcripts, two activating $K I R$ transcripts are present. In this analysis, KIR transcripts were only considered if they represented at least $0.9 \%$ of total reads. If, however, all transcripts identified within individuals were taken into account, the number of KIRs does not change significantly (highest number of KIRs was 12 in individual mm41).

To demonstrate the high variability of interindividual differences of the content of $K I R$ transcripts, three rhesus macaques (mm5, mm6, and mm20) were compared (Figure 11). For each animal the distinct $K I R$ transcripts detected are shown, along with the relative expression level of each KIR. The comparison of three animals already demonstrates the high diversity of different $K I R$ transcription profiles in the cohort. While individual $\mathrm{mm} 20$ possessed ten distinct $K I R$ transcripts, only four and five different $K I R$ transcripts were identified in individual $\mathrm{mm} 5$ and $\mathrm{mm} 6$, respectively. The advantage of 454 sequencing to provide estimates of the relative contribution of each $K I R$ within individuals additionally increases the diversity of unique $K I R$ profiles. Animal mm20 possessed ten KIR transcripts with expression levels comprising $0.9 \%$ to $23.5 \%$ of total reads. Similar observations were made for individual mm6 with three KIR transcripts, KIR3DL01, KIR3DLO8 and KIR3DSO3 expressed at comparable levels of about $25 \%$ to $35 \%$ of total sequencing reads. The dominant expression level of KIR3DL11 transcripts representing approximately $57 \%$ of total reads identified in individual mm5, demonstrates a clear contrast to both of the other examples. The dominant expression of a single inhibitory or activating KIR representing at least $45 \%$ of total reads was documented in several animals. These data confirm findings published by Moreland et al. (2011). 

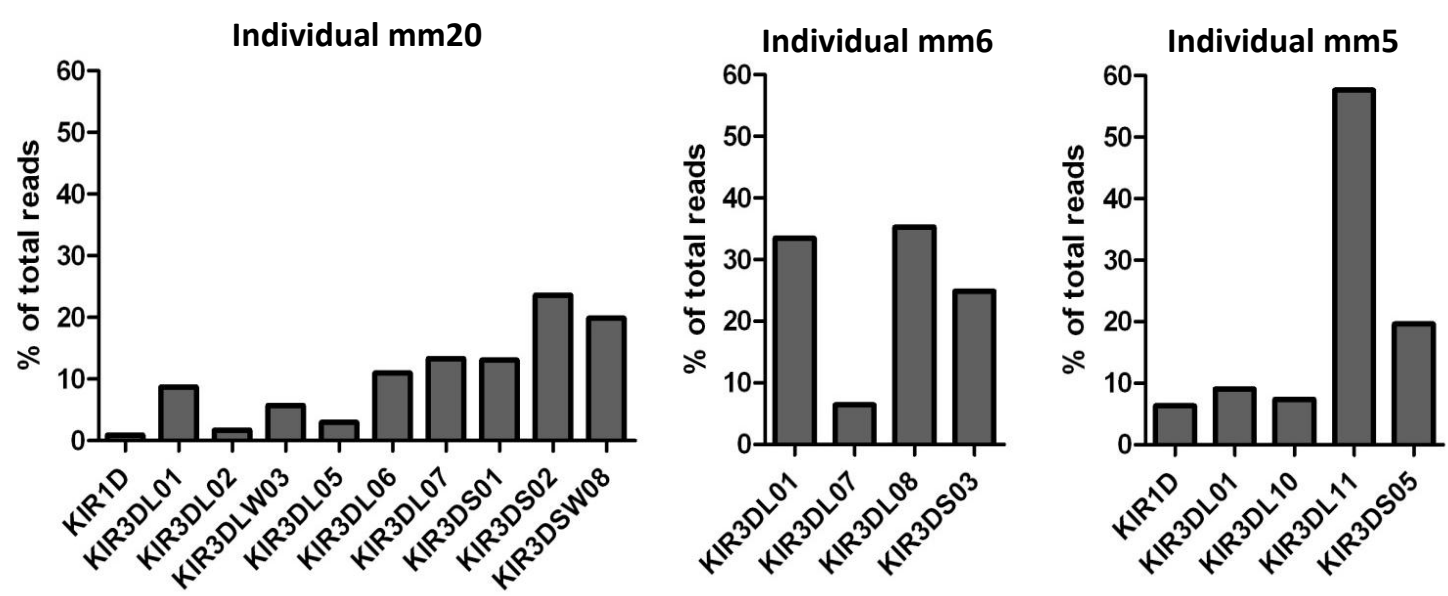

Figure 11 Interindividual differences of three different $K I R$ transcription profiles.

The KIR profiles of transcribed KIR genes, established with 454 sequencing of cDNA-PCR amplicons are shown for three different individuals $(\mathrm{mm} 20, \mathrm{~mm} 6, \mathrm{~mm} 5)$. For each animal, the distinct KIRs detected are shown, along with the relative frequency of each $\operatorname{KIR}(\%$ of total reads).

Seven distinct $K I R$ transcripts were documented to be present in $\geq 50 \%$ of the macaque cohort (Figure 9). A common KIR repertoire, which was detected in 16 individuals, contains transcripts of the inhibitory KIR3DL01, KIR3DL05, KIR3DLO7 and the activating KIR3DSO2. Comparison of KIR transcription profiles of individuals sharing these KIR transcripts still showed considerable differences in expression levels of the shared KIRs, and in many cases individuals expressed additional KIRs. However, it is known that KIR expression is influenced by the KIR and MHC class I genotypes of an individual (Shilling et al., 2002). The considerable differences observed in expression levels of the shared KIRs suggest that differences in the KIR/MHC class I genotypes are evident and, indeed, these individuals are neither completely identical for their KIR nor MHC class I repertoire (7.1; 7.2, Supplement).

\subsubsection{Diversity of $M H C$ class I transcripts in the rhesus macaque cohort}

The most important ligands of KIRs are the MHC class I molecules. In addition to the established KIR transcription profiles, the same rhesus macaque cohort was characterised for MHC class I gene transcripts, by using a next generation sequencing approach of cDNA-PCR amplicons (O'Leary et al., 2009). The analysed amplicon sequences encode for a highly polymorphic region of MHC class I proteins, which is responsible for the binding of antigenic peptides. Based on the high sequence variability of the analysed MHC class I amplicons, it was possible to distinguish between almost all known $M H C$ class I alleles in a very comprehensive way. 
The analysis revealed 74 distinct $M H C$ class $I$ sequences with an average of about 10 different MHC class I transcripts per individual. In the analysed rhesus macaque cohort, individuals were identified to possess at least eight and a maximum of 15 distinct MHC class I transcripts, in which only transcripts comprising $\geq 1 \%$ of total sequencing reads of that individual were taken into account. However, if all transcripts detected within the rhesus macaque cohort were considered to represent a transcribed MHC class I allele independent of the percentage of total sequencing reads, haplotypes comprising up to 18 distinct MHC class I transcripts were documented (7.2, Supplement). These findings support the enormous complexity of $M H C$ class I genotypes that was already known for rhesus macaques (Otting et al., 2005; Wiseman et al., 2009).

Moreover, each MHC class I haplotype was found to comprise at least one Mamu-AI allele, in combination with one to two distinct Мати-A2, $-A 3$, and $-A 4$ alleles, showing the typical presence and absence polymorphisms of these $M H C$ class $I$ alleles (Otting et al., 2007). Analysis of the relative expression levels of $M H C$ class I transcripts ( $\%$ of total reads) showed that individuals dominantly expressed multiple Мати- $A$ and Мати-B alleles, designated as “major" genes (Otting et al., 2005; Otting et al., 2007; Rosner et al., 2010). The majority of Mamu-Al alleles identified make up at least $10 \%$ and up to $59 \%$ of total sequence reads within individuals. Likewise several Мати-B alleles were detected at high expression levels up to $39 \%$ of total reads, like e.g. Мати- $B^{* 002},-B^{*} 004$, and $-B * 048$ (7.2, supplement). Expressed at comparatively low levels and designated as "minor" genes (Otting et al., 2005), alleles of Мати-A2, -A3, -A4 and certain Мати-B alleles, like e.g. Мати-B*051, and $-B * 060$ were documented.

All results of the comprehensive MHC class I profiling of the SIV-infected rhesus macaque cohort are shown in chapter 7.2 (supplement), with individuals divided according to plasma viral loads at set point (HVL and LVL cohort).

\subsubsection{KIR/MHC class I combinations}

The variability of the content of $K I R$ transcripts and the diversity of the relative expression level of each KIR transcript both contribute to the large differences of individual KIR transcription profiles. This diversity complicates the identification of "KIR identical" animals within the macaque cohort. However, five animals (pre infection samples of mm10, mm14, $\mathrm{mm} 23, \mathrm{~mm} 25, \mathrm{~mm} 48)$ are identical for five different KIR transcripts (KIR1D, KIR3DL01, KIR3DL05, KIR3DL10, KIR3DS02, KIR3DS05) with partly comparable expression levels and 
without showing expression of additional KIRs (7.1, supplement). These samples were selected and compared for their MHC class I alleles. Eight to 15 distinct MHC class I transcripts were detected in these individuals if only transcripts comprising at least $1 \%$ of total reads were taken into account. The comparison of the MHC class I profiles of these five individuals did not even identified a single MHC class $I$ allele that was shared by all five individuals examined (7.2, supplement). Only three $M H C$ class $I$ alleles were present in four out of five individuals (Mamu-B*030, $-B * 057,-B * 072)$.

Eight individuals were found to share the same Mamu-Al alleles $(A 1 * 001$ and $A 1 * 004)$, however, these animals were only identical for KIR3DLO1 transcripts, which was detected in $94 \%$ of the cohort anyway (3.2.1), and most of them shared transcripts of KIR3DLO7, KIR3DL08, and KIR3DSO2. However, not even two of these Mamu-Al identical macaques were completely KIR identical.

Recently, specific interactions between KIR3DL05 and Mamu-A1*001, and -A3*13, and between KIR3DLW03 and Mamu-A1*001, were published (Rosner et al., 2011). Thus, individuals with higher expression levels of KIR3DLO5 transcripts ( $\geq 10 \%$ of total reads; $\mathrm{n}=13$ ), were analysed for their Maтu-A1*001 and $-A 3 * 13$ transcription. Eight individuals were positive for $М а т и-A 1 * 001$ and five individuals were positive for Mamu-A3*13, however, the relative expression levels of both Mamu-A alleles were comparable with KIR3DL05-negative individuals that likewise expressed the indicated $M H C$ alleles. As KIR3DLW03 transcripts were mainly found at lower levels, all KIR3DLW03-positive macaques were compared for their Mamu-A1*001 expression, but less than the half of these individuals were positive for this KIR.

Identifying potential ligands for KIR molecules is still challenging due to the high complexity of $M H C$ class $I$ and KIR profiles in rhesus macaques, and KIRs have the ability to recognise multiple MHC class I ligands with a common amino acid motive in their $\alpha 1$ domains (Gumperz et al., 1995; Mandelboim et al., 1996). However, comprehensive profiles of transcribed KIR and MHC class I genes may allow to reduce the number of probable interaction candidates for functional studies. 


\subsection{Investigation of $K I R$ and $M H C$ class I transcripts and viral loads}

The SIV-infected rhesus macaque cohort was divided into two groups based on the plasma viral loads at set point of each rhesus macaque. In consideration of this characteristic, 28 animals were allocated to the high viral load cohort (HVL $>10^{6}$ viral copies/ $\mathrm{ml}$ of plasma), and 24 to the low viral load cohort (LVL $<10^{5}$ viral copies/ $\mathrm{ml}$ of plasma). In order to identify KIR transcripts, which were associated with disease pathogenesis in SIV infection, the established KIR profiles of each rhesus macaque were compared between the HVL and LVL cohort. Significant differences were calculated by using two-tailed Fisher`s exact tests $(p \leq 0.05)$. The pre-processing, filter strategies, and final analysis of 454 sequencing data are described in chapters 2.2.9 (KIR) and 2.2.10 (MHC class I).

Comprehensive KIR transcription profiles were obtained by sequencing pre infection samples. For one individual (mm24) only a post infection sample (acute phase) was included, as a sample prior to infection with SIV was not available. However, this macaque was only taken into account for the analysis of differences in the content of KIR transcripts between both cohorts, and not for the analysis of the relative expression levels of KIRs (explained in 3.2). Equal comparison was performed to detect "favourable" or "disadvantageous" MHC class I transcripts.

\subsubsection{Comparison of the frequencies of transcribed $K I R$ genes according to plasma viral loads}

To identify KIR transcripts, which are associated with disease progression during experimental SIV infection, the frequencies of all KIR transcripts were compared between the HVL and the LVL cohort.

Comparison of the KIR transcript frequencies between the HVL and LVL cohorts revealed two inhibitory KIR transcripts, which were significantly associated $(p \leq 0.05)$ with viral loads. KIR3DL02 transcripts were more frequently found in the LVL cohort $(p=0.022)$, whereas KIR3DL10 transcripts are more frequent in the HVL cohort $(p=0.049)$ (Figure 12). The remaining KIR transcripts are distributed approximately equally between the HVL and LVL cohorts or the differences failed statistical support $(p \geq 0.05)$. The percentage of animals positive for $K I R$ transcripts and the calculated $p$-values for each $K I R$ transcript are listed in Table 8. Pre infection samples were considered in the analysis only. 


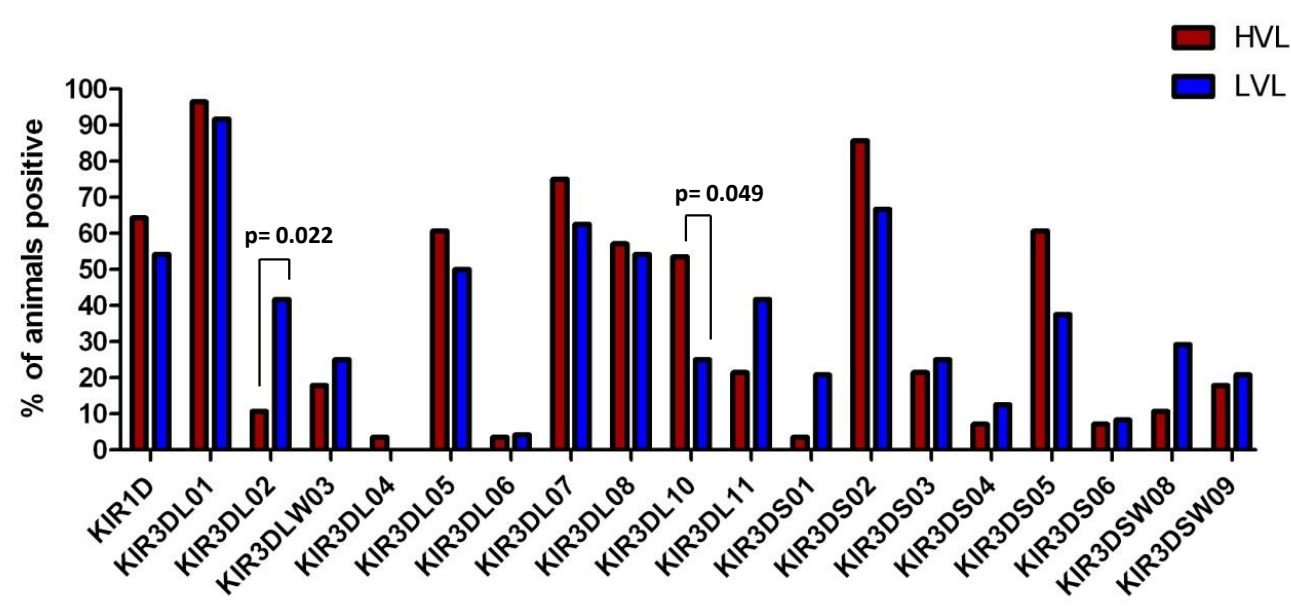

Figure 12 Frequencies of KIR transcripts in the HVL and LVL cohort (pre infection*).

The y-axis indicates the percentage of animals within the two cohorts that express the indicated KIR. The animals of the HVL cohort $(\mathrm{n}=28)$ are shown in red and animals of the LVL cohort $(\mathrm{n}=24)$ are shown in blue. Only samples prior to infection with SIV were included (*mm24 acute infection sample). In order to reduce errors introduced by PCR, KIR transcripts were only considered to be present if they comprise $\geq 0.9 \%$ of total sequencing reads of that animal. Significant differences between both cohorts were calculated using two-tailed Fisher`s exact test. KIR3DLO2 and KIR3DL10 expression are significantly associated with viral loads ( $p$-values are shown), while the remaining KIRs are distributed equally between the two cohorts.

Table 8 Frequencies of transcribed KIR genes in the HVL and LVL cohort (pre infection)

\begin{tabular}{|c|c|c|c|c|c|}
\hline \multirow[b]{2}{*}{ KIR } & \multicolumn{2}{|c|}{ HVL cohort $\left(n=28^{* *}\right)$} & \multicolumn{2}{|c|}{ LVL cohort $(n=24)$} & \multirow[b]{2}{*}{$p$-value } \\
\hline & $\#^{a}$ & $\%^{b}$ & $\#^{a}$ & $\%^{b}$ & \\
\hline $1 \mathrm{D}$ & 18 & 64.3 & 13 & 54.2 & 0.574 \\
\hline 3DL01 & 27 & 96.4 & 22 & 91.7 & 0.59 \\
\hline 3DL02 & 3 & 10.7 & 10 & 41.7 & $0.022 *$ \\
\hline 3DLW03 & 5 & 17.9 & 6 & 25.0 & 0.735 \\
\hline 3DL04 & 1 & 3.6 & $n / d$ & $n / d$ & $\mathrm{n} / \mathrm{d}$ \\
\hline 3DL05 & 17 & 60.7 & 12 & 50.0 & 0.577 \\
\hline 3DL06 & 1 & 3.6 & 1 & 4.2 & 1.0 \\
\hline 3DL07 & 21 & 75.0 & 15 & 62.5 & 0.378 \\
\hline 3DL08 & 16 & 57.1 & 13 & 54.2 & 1.0 \\
\hline 3DL10 & 15 & 53.6 & 6 & 25.0 & 0.049* \\
\hline 3DL11 & 6 & 21.4 & 10 & 41.7 & 0.141 \\
\hline 3DS01 & 1 & 3.6 & 5 & 20.8 & 0.084 \\
\hline 3DSO2 & 24 & 85.7 & 16 & 66.7 & 0.186 \\
\hline 3DSO3 & 6 & 21.4 & 6 & 25.0 & 1.0 \\
\hline 3DS04 & 2 & 7.1 & 3 & 12.5 & 0.652 \\
\hline 3DS05 & 17 & 60.7 & 9 & 37.5 & 0.164 \\
\hline 3DS06 & 2 & 7.1 & 2 & 8.3 & 1.0 \\
\hline 3DSW08 & 3 & 10.7 & 7 & 29.2 & 0.157 \\
\hline 3DSW09 & 5 & 17.9 & 5 & 20.8 & 1.0 \\
\hline
\end{tabular}

${ }^{\text {a }}$ Total numbers of animals positive for the indicated $K I R$ transcript

${ }^{\mathrm{b}}$ Percentage of animals positive for the indicated $K I R$ transcript

$* p \leq 0.05$

** mm24, acute infection sample

$\mathrm{n} / \mathrm{d}=$ not detected or determined, because no data available 
In addition to KIR3DLO2 and KIR3DL10 transcripts, which are associated with low and high viral loads (Figure 12), respectively, other KIR transcripts were shown to be more frequent in one of the two cohorts. The transcripts of the inhibitory KIR3DL11 for example, were identified in 10 animals of the LVL cohort and in six animals of the HVL cohort, and were by trend associated with low viral loads, but failed statistical support $(p=0.141)$. In the studied cohort no activating KIR transcripts were associated with viral loads. Nevertheless, the activating KIR3DSO1 was more frequently identified in the low viral load cohort compared to animals with high viral loads, but this difference slightly failed statistical support $(p=0.084)$. However, due to the low number of KIR3DS01-positive animals (HVL, n=1; LVL, n=5) more individuals need to be characterised. In addition to KIR3DSOI transcripts, the activating KIR3DSO2 and KIR3DSO5 were both more frequently detected in the HVL cohort, and transcripts of KIR3DSW08 were more frequently observed in the LVL cohort, but failed to be statistically significant (Table 8).

\subsubsection{Relative expression levels of KIR transcripts in the HVL and LVL cohort (pre infection)}

A key advantage of 454 sequencing is that it provides estimates of the relative transcription levels of $K I R$ genes. In addition to the observed differences in the frequencies of transcribed $K I R$ genes in the HVL and LVL cohort (3.3.1), differences in the relative expression levels of all KIRs identified in individuals of the HVL and LVL cohort, were investigated. Therefore pre infection samples were used (individual mm24 was excluded). This analysis should give information on the influence of KIR transcription levels with regard to disease pathogenesis in SIV infection, similar to what was shown for specific KIR3DL1 alleles in humans. These KIR3DL1 allotypes in human were shown to have a protective effect in HIV-1 infection particularly if they are expressed at high levels (Martin et al., 2007).

The relative expression levels of most KIRs detected in the HVL and the LVL cohort showed no significant or only marginal differences between the two cohorts (Figure 13). Moreover, testing statistical differences between individuals was impossible if less than two data points (two positive individuals) were available within one of the compared cohorts, like KIR3DSOI. All calculated $p$-values are listed in Table 9. 


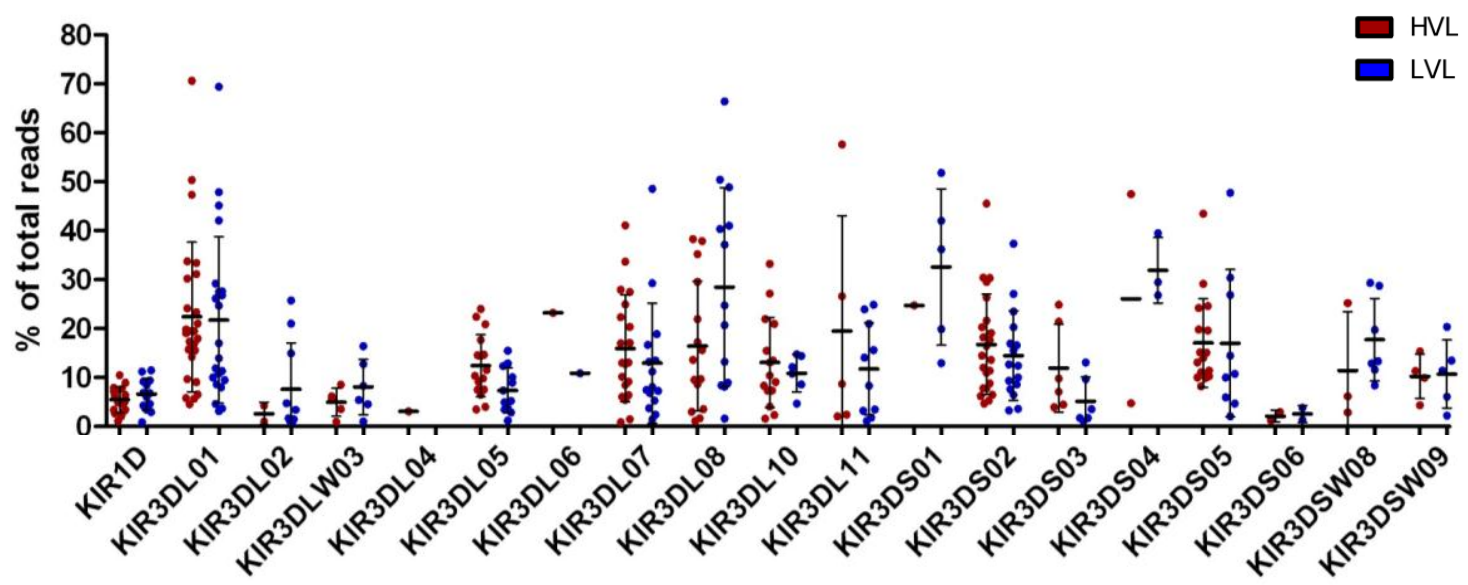

Figure 13 Comparison of the relative transcription levels of KIRs in the HVL and LVL cohort (pre infection).

The $y$-axis indicates the percentage of total sequencing reads per individual for each KIR transcript identified. The animals of the HVL cohort are shown in red $(\mathrm{n}=27)$ and animals of the LVL cohort are shown in blue $(\mathrm{n}=24)$. Only samples prior to infection with SIV were included (mm24 was excluded). Averages and SD are represented by bars.

Table 9 Differences of the relative expression levels of KIRs between the HVL and LVL cohort (pre infection)

\begin{tabular}{llll}
\hline \hline KIR & $\boldsymbol{p}$-value & KIR & $\boldsymbol{p}$-value \\
\hline 1D & 0.304 & 3DS01 & $\mathrm{n} / \mathrm{d}$ \\
3DL01 & 0.896 & 3DS02 & 0.481 \\
3DL02 & 0.488 & 3DS03 & 0.138 \\
3DLW03 & 0.296 & 3DS04 & 0.751 \\
3DL04 & n/d & 3DS05 & 0.990 \\
3DL05 & $\mathbf{0 . 0 2 8 *}$ & 3DS06 & 0.778 \\
3DL06 & n/d & 3DSW08 & 0.364 \\
3DL07 & 0.448 & 3DSW09 & 0.909 \\
3DL08 & 0.071 & & \\
3DL10 & 0.591 & & \\
3DL11 & 0.37 & & \\
\hline
\end{tabular}

$* p$-value $\leq 0.05$

$\mathrm{n} / \mathrm{d}=$ not determined, because less than 2 data points available

The comparison of the relative expression levels between both cohorts revealed significantly different transcription levels of KIR3DL05. Figure 14 shows that the transcripts of KIR3DL05 are consistently expressed at higher levels in animals of the HVL cohort, with an average of $12.42 \%$ of total sequencing reads, compared to the LVL cohort with an average of $7.39 \%$ of total sequencing reads $(p=0.028)$. The frequencies of KIR3DLO5 transcripts were distributed equally between the HVL and LVL cohort $(p=0.577)$ (Table 8). 


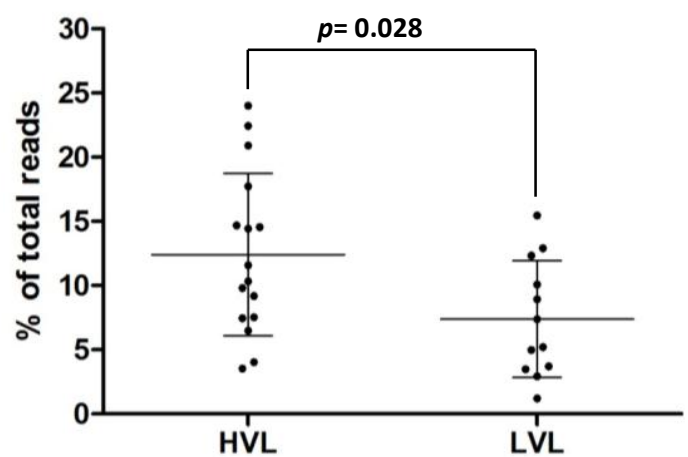

Figure 14 Comparison of KIR3DLO5 transcription levels between the HVL and LVL cohort (pre infection).

Each dot indicates the relative contribution of KIR3DLO5 transcripts to the total of sequencing reads identified in each animal. Bars representing the mean and SD (HVL: $n=16$, mean $12.42 \%$ of total reads; LVL: $\mathrm{n}=12$, mean $7.39 \%$ of total reads) of KIR3DL05 transcription levels. The comparison indicates that higher KIR3DLO5 transcription levels are significantly associated with high viral replication. Only transcripts comprising $\geq 0.9 \%$ of total reads were included. Significant differences of KIR3DL05 transcription level between both cohorts were tested by performing two-tailed t-test.

\subsubsection{Comparison of KIR3DLO8 transcription between the HVL and LVL cohort}

Higher expression levels of KIR3DL08 transcripts were identified in animals with low viral loads (mean $27.96 \%$ of total reads) compared to the HVL cohort (mean $17.11 \%$ of total reads). However, the observed difference failed to be statistically significant ( $p=0.071)$, due to the high variability of KIR3DLO8 expression levels between subjects (Figure 13, Table 9). Within the LVL cohort 12 out of 24 rhesus macaques satisfy the criteria of elite controllers (EC), as they maintained low or undetectable plasma viral loads ( $<50$ copies/ $\mathrm{ml}$ ) for more than one year without antiviral therapy. If $K I R 3 D L 08$-positive animals $\left(K I R 3 D L 08^{+}, \mathrm{n}=28\right)$ were divided in EC (n=9) and non-EC ( $n=19)$, it becomes obvious that significantly higher expression levels of KIR3DLO8 transcripts (non-EC mean $15.28 \%$, EC mean $35.52 \%$ ) are found in ECs $(p=0.002)$ (Figure 15). Moreover, only three out of 12 ECs are negative for KIR3DL08 transcripts. 


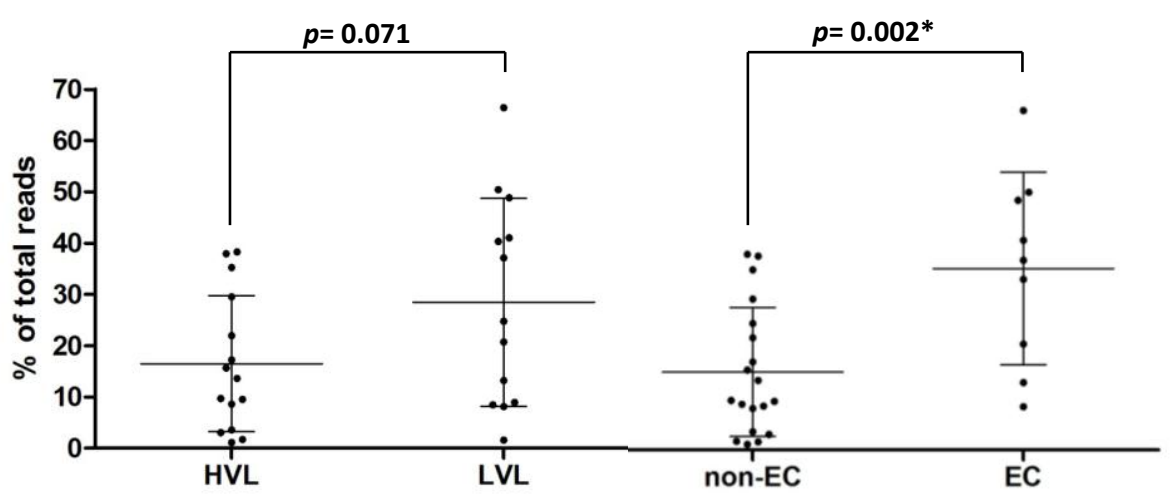

Figure 15 Comparison of KIR3DLO8 transcription in $K I R 3 D L O 8^{+}$individuals (pre infection). The y-axis indicates the percentage of total sequencing reads of KIR3DLO8 transcripts within each individual. Bars representing the mean and SD. On the left panel $K I R 3 D L O 8^{+}$animals were divided in the HVL $(n=15)$ and LVL $(n=13)$ cohort, indicating that the difference between both cohorts slightly failed statistical support $(p \geq 0.05)$. On the right panel KIR3DLO $8^{+}$animals were divided in non-EC $(\mathrm{n}=19)$ and $\mathrm{EC}(\mathrm{n}=9)$ animals, indicating significant differences $\left({ }^{*} p \leq 0.05\right)$ of KIR3DLO8 expression levels $(p=0.002)$ between both groups. Statistical differences of KIR3DL08 transcription levels among the distinct groups were tested by performing two-tailed t-tests.

The relative expression levels of $K I R$ transcripts are expected to be influenced by the complete KIR/MHC class I genotype. However, the ligand of KIR3DL08 is not known so far. Thus, all KIR3DLO ${ }^{+} \mathrm{EC}$ and non-EC were compared for their MHC class I transcripts but no obvious similarities within $K I R 3 D L 08^{+} \mathrm{EC}$, or differences between $K I R 3 D L 08^{+} \mathrm{EC}$ and nonEC, were identified.

\subsubsection{Comparison of MHC class I transcript profiles according to plasma viral loads}

To investigate associations of $M H C$ class I transcripts with viral loads the established $M H C$ class I transcript profiles (3.2.4) of the rhesus macaque cohort were divided according to plasma viral loads at set point. The frequencies of HVL and LVL animals, which were positive for each distinct $M H C$ class I transcript were compared, and significant differences were calculated using Fisher`s exact test.

The Maтu-AI*004 allele was identified in MHC class $I$ haplotypes to be associated with fast SIV-disease progression (Sauermann et al., 2008). In the studied cohort, Maтu-A1*004 transcripts were found more frequently in the HVL cohort $(n=17)$, compared to the LVL cohort $(\mathrm{n}=7)(p=0.029)$. The Мати-Al*001 allele, which is associated with long-term survival and low viral loads upon experimental SIV infection (Miller et al., 1991; Mühl et al., 2002; Zhang et al., 2002; O'Connor et al., 2003), was by trend more frequent in the LVL 
cohort, but failed statistical support $(p=0.163)$. In addition to Mamu-Al*004, transcripts of Mamu-A1*041 were more frequently identified in the LVL cohort $(\mathrm{n}=4)$ compared to the HVL cohort $(\mathrm{n}=0)(p=0.039)$. This association should be considered with caution, based on the small number of animals positive for the indicated MHC class I transcript and should be confirmed by characterising more SIV-infected rhesus macaques for their MHC class I transcription. While relevant differences of Мати-Al alleles were identified between the two cohorts, no Мати-B allele showed statistically significant differences in the detected frequencies between the HVL and LVL cohort. Мати- $B * 08$ and $М а т и-B * 17$, which were both associated with slower progression to AIDS (Loffredo et al., 2007; Yant et al., 2006), were detected in only two $\left(\right.$ Мати- $\left.B^{*} 08, p=0.208\right)$ and in three animals $\left(\right.$ Maтu- $B^{*} 17$, $p=0.092$ ) of the LVL cohort, respectively. It should be noted that the used approach of sequencing cDNA-PCR amplicons will identify transcribed MHC class I genes only, while the studies by Loffredo et al. (2007) and Yant et al. (2006) employed sequence-specific PCR from genomic DNA.

\subsection{Changes in KIR transcription upon SIV infection}

A main focus of this work was to investigate significant changes in the $K I R$ transcript expression at different time points of experimental SIV infection in rhesus macaques. Animals were checked for loss and gain of KIR gene transcription, as well as for differences in the relative expression levels of KIR transcripts over the course of SIV infection. Three samples of PBMCs of each rhesus macaque were characterised for KIR by 454 sequencing of cDNAPCR amplicons. The first sample was obtained prior to infection with SIV (pre), the second sampling point was approximately 2-8 weeks post infection (acute), and the last sample was obtained 12 weeks post infection at the earliest (chronic). The list of all individuals with information on viral loads (peak and set point) is shown in 2.1.1.

It should be mentioned that due to the rapid development of AIDS for HVL animals, the numbers of NK cells in the used PBMC samples was low (personal communication with Professor Aftab Ansari). Although amplification of KIR cDNA-PCR amplicons was possible, the 454 sequencing approach does not provide information on the quality of samples. For this reason the data obtained with chronic infection samples of HVL individuals should be considered with caution, and mainly the changes of KIR transcription during acute SIV infection were of interest. 
Three different sampling points (pre, acute, chronic) of 32 individuals were analysed, whereas for 12 animals only two samples (either pre/acute or pre/chronic) were available. In total 44 animals were taken into account for the analysis, in which 24 animals belong to the HVL cohort ( 3 samples: $\mathrm{n}=17,2$ samples: $\mathrm{n}=7$ ) and 20 belong to the LVL cohort ( 3 samples: $\mathrm{n}=15$, 2 samples: $n=5)$. The pre-processing, filter strategies, and final analysis of 454 sequencing data are described in chapter 2.2.9.

\subsubsection{SIV-dependent changes of the relative expression levels of KIR transcripts}

The investigation of differences in presence and absence of $K I R$ transcripts over the course of infection, thus, in the KIR repertoire within each analysed individual, revealed no significant changes. Only if KIR transcripts are expressed at low levels, like comprising about less than $3 \%$ of total sequencing reads, it occurred that transcripts could not be identified in all three or two compared samples, respectively. Within two individuals only transcripts of KIR3DL11 were identified to comprise $8.7 \%$ (mm26, pre infection sample), and $5.6 \%$ (mm31, acute infection sample) of total sequence reads, but failed to be detected in the other sampling points of these individuals. Moreland et al. (2011) already showed that KIR transcripts expressed at low levels could not be detected in all samples of an animal. However, the verified reproducibility of $K I R$ expression levels along with the KIR transcription profiles of the remaining 42 macaques over the course of disease (7.1; supplement) indicated that the loss or gain of KIR transcripts upon SIV infection within an individual is unlikely and missing KIR transcripts, which are expressed at low levels, are most likely caused by sequencing.

The comparison of the relative expression levels of all KIR transcripts in the cohort of 44 animals revealed two KIR transcripts, KIR3DL05 and KIR3DL07, which showed significant differences during SIV infection (Figure 16). The relative expression levels of KIR3DLO5 are significantly increased during the acute phase of infection $(p=0.006, \mathrm{n}=22)$, compared to pre infection samples (Figure 16A). Moreover, the relative expression levels of KIR3DLO7 are significantly reduced during the acute phase of SIV infection, in comparison with pre infection samples $(p=0.001, \mathrm{n}=26$ ) (Figure 16B). Both KIR transcripts showed no significant differences in the relative expression levels between the acute and the chronic phase of infection. 
A
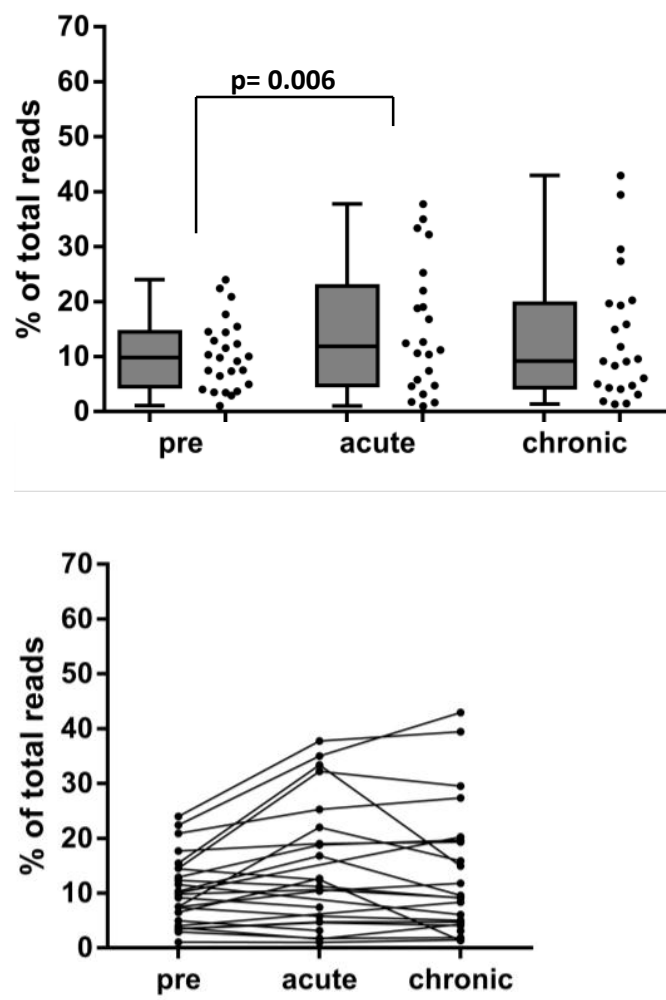

B

KIR3DL07

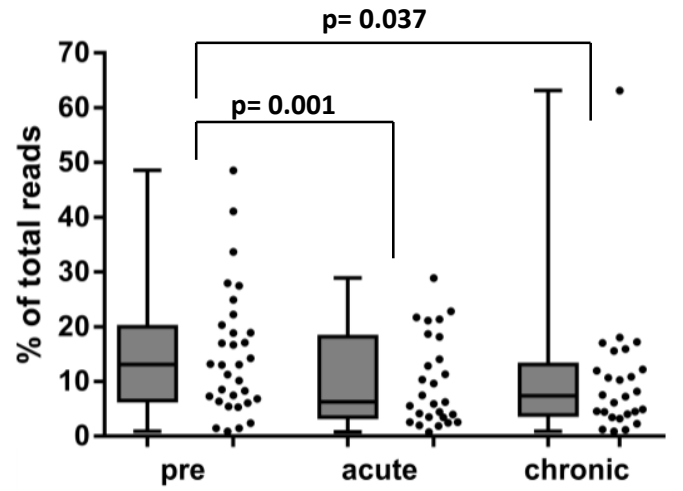

Figure 16 Changes of KIR3DL05 and KIR3DL07 transcription levels in the course of SIV infection.

(A) Changes of the relative transcription levels of KIR3DL05 over the course of SIV disease are shown as box-whisker-plots and dot plots, which represent the transcription levels of KIR3DL05 of single animals. Below the KIR3DLO5 transcription levels are shown as before-after graph to illustrate individual changes over the course of SIV disease. (B) The relative contribution of KIR3DL07 transcripts within animals over the course of SIV infection are shown accordingly. Samples of PBMCs were obtained before infection (pre), approximately 2 weeks after infection (acute), and 12 weeks after infection at the earliest (chronic). Differences of the relative expression levels of both KIR transcripts were calculated by performing paired t-tests. Significant $p$-values $(p \leq 0.05)$ are shown only.

Additionally, the relative transcription levels of the activating KIR3DSO2 were identified to decrease over the acute phase of infection $(p=0.005, \mathrm{n}=30)$, and showed a tendency to increase during the chronic infection $(p=0.076, \mathrm{n}=25)$, compared to the acute phase of SIV infection. However, the difference between pre and acute infection samples is comparatively low, as it was calculated to be $2.89 \%$ on average per individual. 


\subsubsection{SIV-dependent changes of the relative KIR transcription are associated with high viral loads}

In order to identify if the observed changes of KIR3DLO5 and KIR3DLO7 transcription levels (3.4.1) are linked to plasma viral loads, the rhesus macaque cohort was divided into the HVL and LVL classification. The separate analysis revealed that the observed differences in the relative expression levels of KIR3DLO5 and KIR3DLO7 transcripts during the acute phase of infection are significantly associated with high viral loads. Increased KIR3DLO5 transcription levels during the acute phase of SIV infection were more frequently observed in the HVL cohort $(p=0.006, \mathrm{n}=13)$ than in the LVL cohort $(p=0.40, \mathrm{n}=9)$ (Figure 17A). Similarly, the reduced transcription levels of KIR3DLO7 were observed during the acute phase of infection, and this decrease is only significant in the HVL cohort ( $p=0.001, \mathrm{n}=16)$, compared to the LVL cohort ( $p=0.397, \mathrm{n}=10$ ) (Figure 17B). The comparison of the relative expression levels of both $K I R$ transcripts showed no significant differences between the acute and chronic phase of infection in both cohorts.
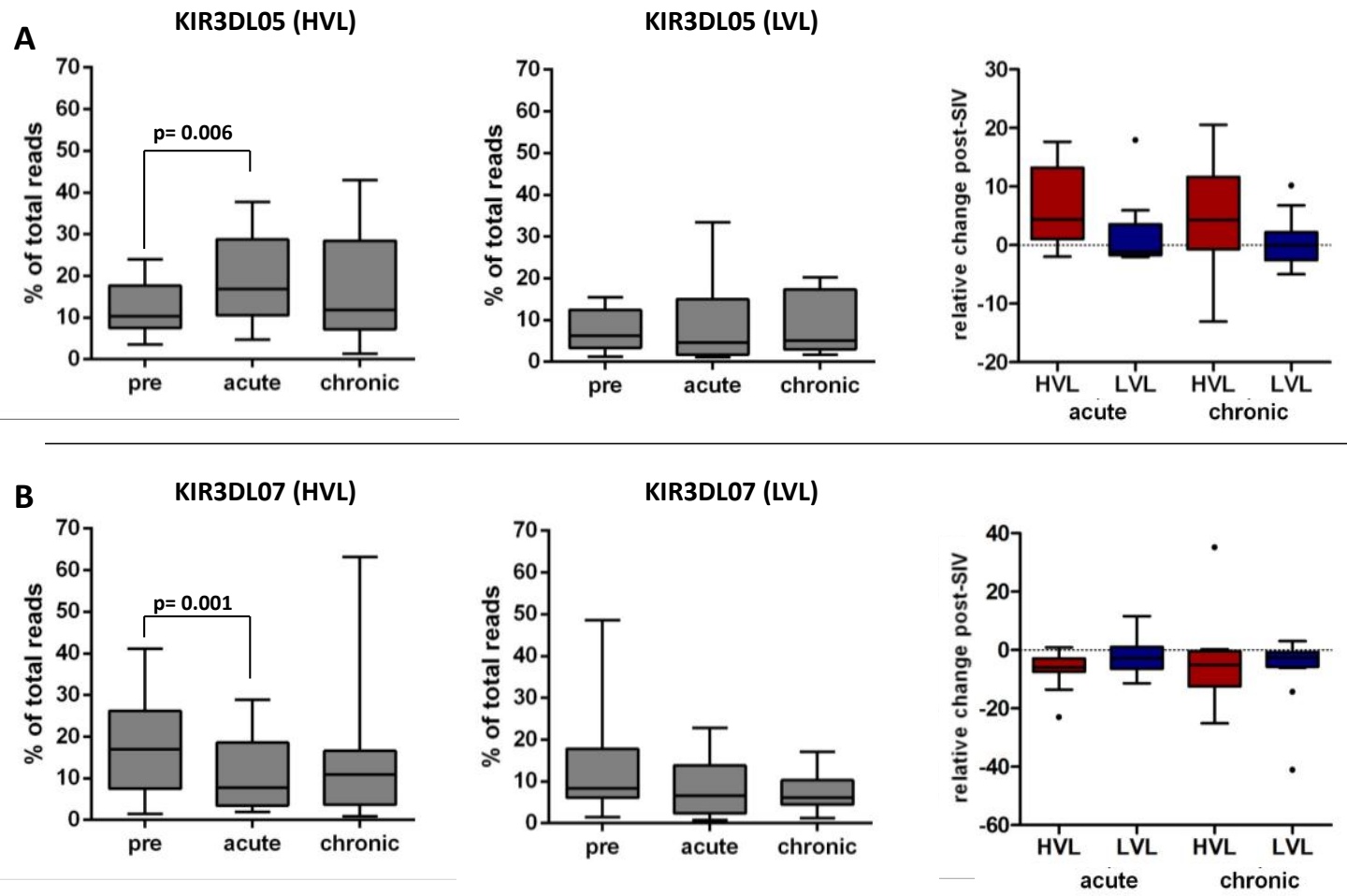

Figure 17 Changes of KIR3DL05 and KIR3DL07 transcription levels in the HVL and LVL cohort over the course of SIV infection.

Changes of the relative transcription levels of (A) KIR3DL05 and (B) KIR3DL07 over the course of SIV disease are shown as box-whisker-plots. On the left panel the changes within the HVL cohort are shown and on the middle panel the observed differences within the LVL cohort are shown, respectively. Differences of the relative expression levels over the course of disease of both KIR transcripts were calculated by performing paired t-tests. Significant $p$-values $(p \leq 0.05)$ are shown only. On the right panel the comparison of the relative changes post infection (\% of total reads) are shown between both cohorts in the acute and chronic phase of infection. Outliers are shown as dots. 
In addition to KIR3DLO5 and KIR3DLO7, significant changes of the relative expression levels of three further KIRs were identified. Similar to the changes of KIR3DL05 transcripts, increased expression levels of KIR3DLO1 during the acute phase of infection, were found in the HVL cohort ( $p=0.043, \mathrm{n}=21)$, compared to the LVL cohort $(p=0.991, \mathrm{n}=15)$ (Figure 18A). Comparison between pre/chronic infection and acute/chronic infection revealed no significant differences.

In chapter 3.3.3 it was shown that higher expression levels of KIR3DLO8 transcripts are more frequently detected in the LVL cohort, compared to animals with high viral loads $(p=0.080)$, and particularly in elite controller animals $(p=0.002)$. During the course of SIV disease the relative KIR3DL08 transcription levels of animals with low viral loads are not changed significantly (Figure 18B). However, SIV-dependent changes of the relative expression levels of KIR3DLO8 were observed in animals of the HVL cohort. The transcription levels are significantly decreased post infection with SIV and particularly during the chronic phase of infection if compared with samples prior to infection $(p=0.030, \mathrm{n}=10)$. These findings, in conjunction with higher expression levels particularly observed in elite controller animals, support the assumption that higher expression levels of KIR3DLO8 are associated with slower disease progression in experimental SIV infection.

Decreased transcription levels of the activating KIR3DSO2 were found in the HVL cohort during transition from pre infection to the acute phase $(p=0.037, \mathrm{n}=19)$, and slightly increased from acute to chronic phase of experimental infection $(p=0.032, \mathrm{n}=15)$. The relative transcription levels of KIR3DSO2 in the LVL cohort were by trend also decreased during the acute phase of SIV infection compared to pre infection samples, and increased during the chronic phase of infection compared to acute phase of infection, but all comparisons failed statistical support (Figure 18C). 
A

KIR3DL01 (HVL)

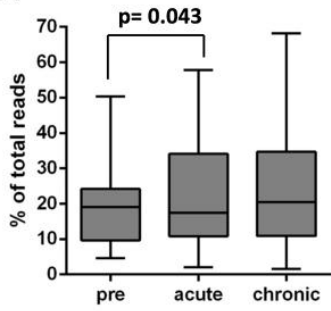

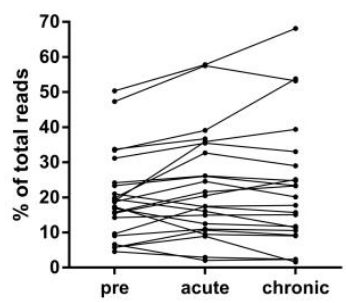

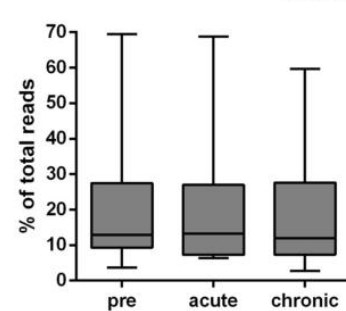

KIR3DL01 (LVL)

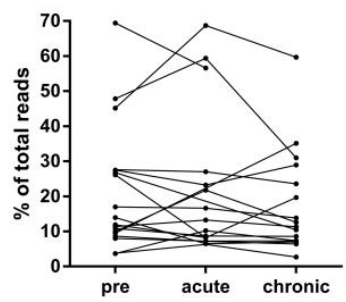

B

KIR3DL08 (HVL)
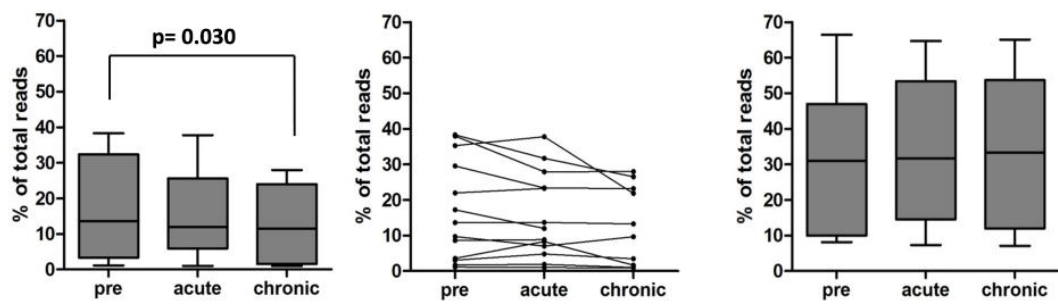

KIR3DL08 (LVL)
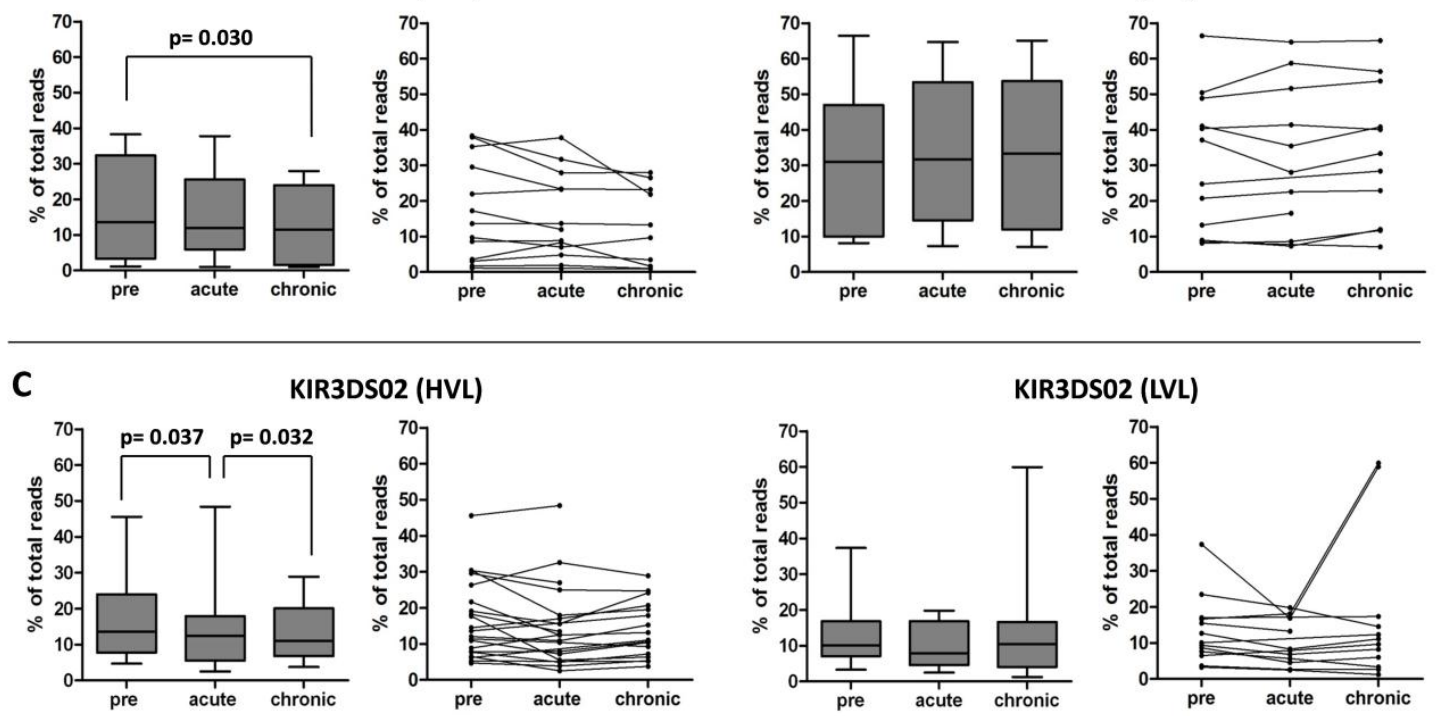

KIR3DS02 (HVL)
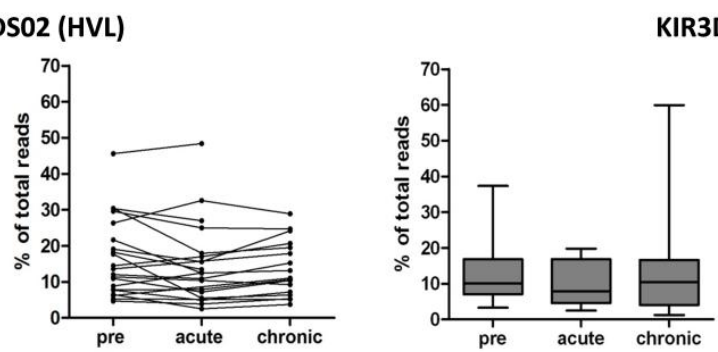

KIR3DS02 (LVL)

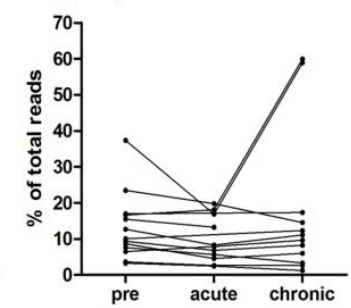

Figure 18 SIV-dependent changes of KIR transcription levels in the HVL and LVL cohort.

Changes of the relative transcription levels of (A) KIR3DLO1, (B) KIR3DLO8, and (C) KIR3DSO2 over the course of SIV disease are shown as box-whisker-plots and as before-after graph to illustrate individual changes. On the left panel the changes within the HVL cohort are shown and on the right panel the observed differences within the LVL cohort are shown, respectively. Differences of the relative expression levels of each $K I R$ transcript were calculated by performing paired t-tests. Significant $p$-values $(p \leq 0.05)$ are shown only.

In conclusion it was shown that the relative expression levels of four different inhibitory KIRs (KIR3DL01, KIR3DL05, KIR3DL07, KIR3DL08) and one activating KIR (KIR3DSO2) were influenced by experimental SIV infections in the cohort of rhesus macaques. However, the most relevant SIV-dependent differences have been observed for the relative expression levels of KIR3DL05 transcripts, which are increased in the acute phase of SIV infection, and KIR3DL07 transcripts, which were significantly decreased during the acute phase of SIV infection. Moreover, all observed changes are associated with higher rather than lower viral loads. The results obtained with samples of LVL animals showed the same trend to some extent, except for the changes of KIR3DL01 and KIR3DL08 transcription levels, but these results failed statistical support. These findings suggest that the relative rates of KIR gene transcription do not change over the course of experimental SIV infection in those rhesus macaques that show low levels of virus replication and slow disease progression. 


\subsubsection{Inverse correlation of KIR3DL05 and KIR3DLO7 transcription levels}

In chapter 3.4.1 the changes of the relative transcription levels of KIR3DL05 and KIR3DLO7 over the course of the experimental SIV infection were described. While transcription levels of KIR3DL05 were significantly increased during the acute phase of infection $(p=0.006$, $\mathrm{n}=27$ ), the relative expression levels of KIR3DLO7 transcripts showed a considerable decrease ( $p=0.001, \mathrm{n}=32$ ), compared with samples prior to infection with SIV, respectively. Both KIR transcripts were detected to be frequently expressed together within individuals (21 out of 52). To identify simultaneous increase and decrease of the relative expression levels of both KIR transcripts, correlation analyses were performed. The relative expression levels ( $\%$ of total reads) of both KIR transcripts were normalised ( $\frac{\text { acute-pre }}{\text { mean (pre;acute) }}$ or $\frac{\text { chronic-pre }}{\text { mean (pre;chronic) }}$ ).

The normalised values of KIR3DLO5- and KIR3DLO7-positive individuals of either pre and acute infection samples, or pre and chronic infection samples, were incorporated in the correlation analysis. Estimates of the regression line were calculated using a linear regression. The analysis revealed that the pre/acute infection samples (Pearson $r=-0.58, p=0.015, \mathrm{n}=17$ ) (Figure 19A) and the pre/chronic infection samples (Pearson $r=-0.63, p=0.007, \mathrm{n}=17$ ) (Figure 19B) are both correlated. Raw data used for normalisation and correlation analysis are shown in 7.1 (supplement). The analysis has shown that the observed increase of KIR3DL05 transcription levels and the decrease of KIR3DLO7 transcription levels are correlated inversely during the acute and chronic phase of SIV infection, respectively. In a minority of individuals decreased KIR3DLO5 transcription levels were documented in combination with increased KIR3DL07 transcription levels. As an example of non-correlating expression levels between pre and acute infection samples, KIR3DLO1 and KIR3DLO5 transcripts were arbitrarily chosen for the correlation analysis (Figure 19C). 

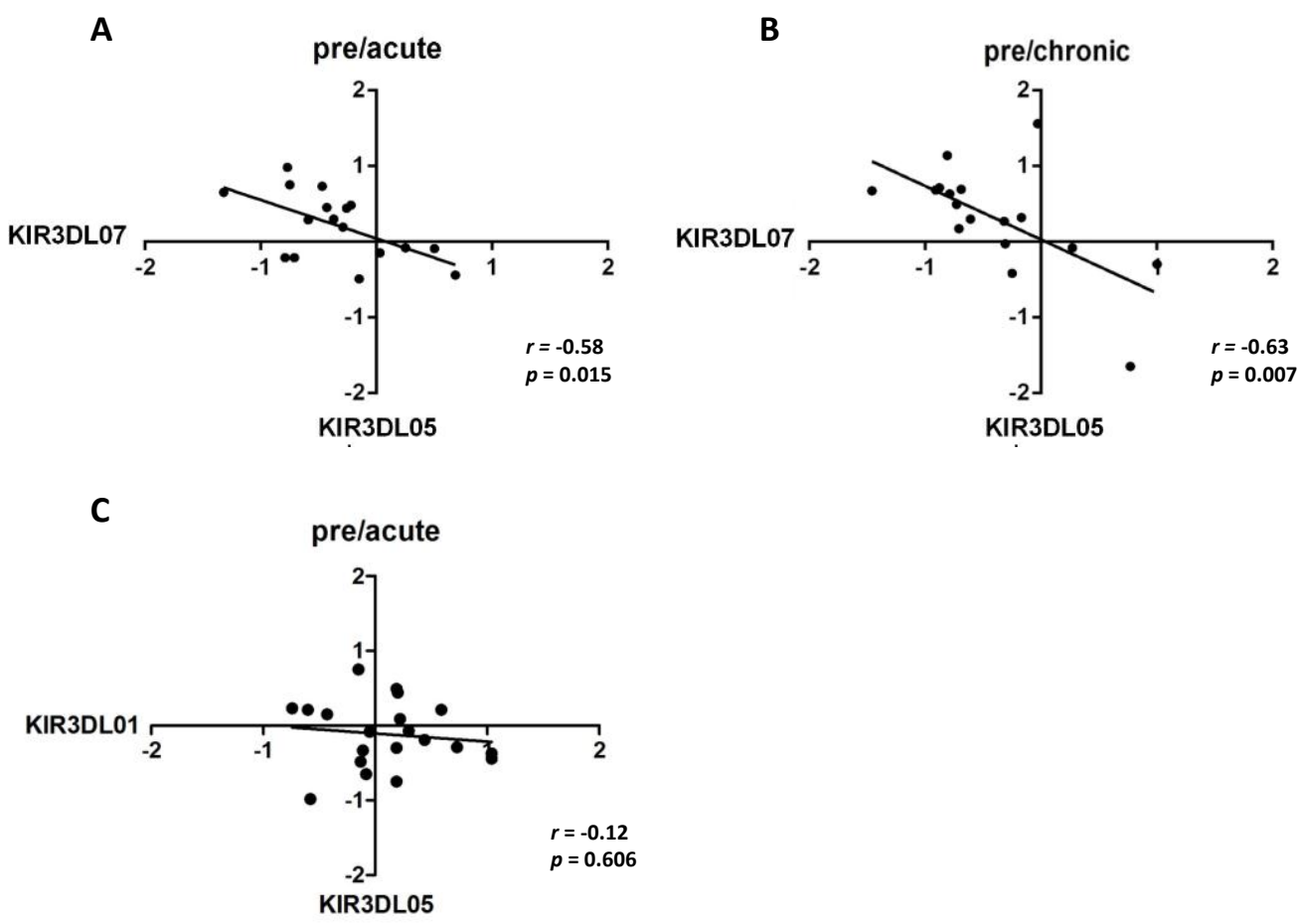

Figure 19 Correlation analysis of KIR3DL05 and KIR3DL07 (KIR3DL01) transcription levels. The estimated relative expression levels of KIR3DL05, KIR3DLO7 and KIR3DL01 transcripts were normalised. (A, B) The normalised values of KIR3DLO7 transcription levels are plotted on the $\mathrm{X}$-axis, in which negative values represent decreased expression levels of that KIR. The normalised values of KIR3DL05 transcription levels are plotted on the y-axis, in which positive values indicate increased expression levels. (A) The correlation between pre and acute infection samples of KIR3DL05 and KIR3DL07 is shown (Pearson $r=-0.58, p=0.015, \mathrm{n}=17$ ) and $(\mathrm{B})$ the correlation between pre and chronic infection samples (Pearson $r=-0.627, p=0.007, \mathrm{n}=17$ ) is shown. Estimates of the regression line were calculated using linear regression analysis. (C) The correlation between pre and acute infection samples is shown (Pearson $r=-0.12, p=0.606, \mathrm{n}=20$ ), indicating that the expression levels of both transcripts are not correlated.

\subsubsection{Investigation of interindividual changes of $K I R$ transcription levels over the course of SIV infection}

So far changes of the relative expression levels of certain KIR transcripts were identified both over the course of SIV infection within the rhesus macaque cohort and between HVL and LVL cohorts (3.4). However, the mean increase of the relative expression levels documented between pre and acute infection samples of each individual of e.g. KIR3DL05 transcripts in the HVL cohort (Figure 17) was $6 \%$ of total sequencing reads per individual. Nevertheless, the large number of animals showing comparable changes of the relative expression level of KIR3DL05 contributes to the significant differences detected during the course of SIV disease. In addition to these findings, individuals were analysed separately to investigate significant changes of the relative expression levels of single KIR transcripts within an 
individual, independent of the changes observed within the total cohort of rhesus macaques. Substantial increases or decreases in expression levels of single $K I R$ transcripts within an individual, comprising $\geq 10 \%$ of total sequencing reads between different stages of SIV infection, were of interest. Therefore individuals that were sampled at all three points in time over the course of SIV infection were selected for the analysis.

A fraction of the rhesus macaque cohort showed no large changes ( $\leq 10 \%$ of total reads) of the relative expression levels of KIR transcripts, regardless of whether they belong to the HVL or LVL cohort, respectively. For instance, the relative expression levels of KIR transcripts identified in individuals mm36 and mm46 are shown in Figure 20. The relative expression levels of all KIR transcripts identified within these two individuals are not changed, if compared between pre, acute, and chronic phase of infection.
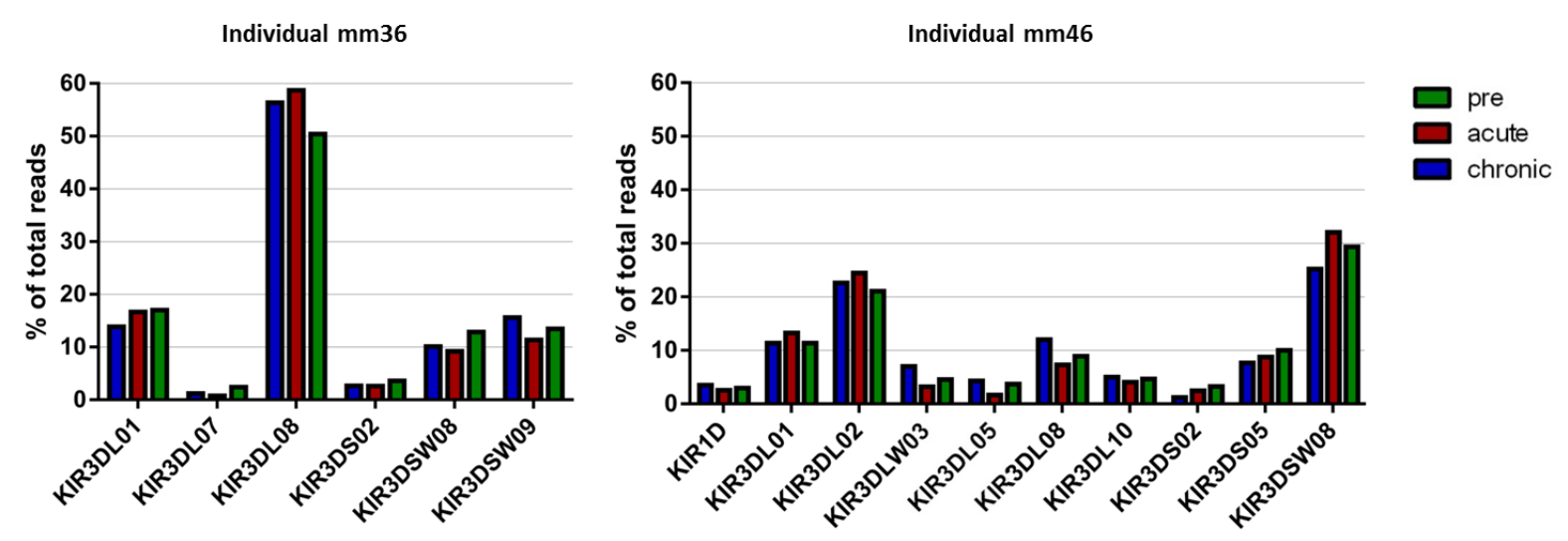

Figure 20 Comparison of constant KIR transcription profiles over course of SIV infection.

The $y$-axis indicates the percentage of total sequencing reads per individual for each KIR transcript identified. The comparison of the KIR transcripts profiles established with three different samples are shown for two individuals (mm36, right; mm46, left).

In contrast some individuals showed striking differences of the relative expression levels of single KIR transcripts over the course of SIV infection. In individual mm32 (LVL) the relative expression levels of the activating KIR3DSO2 are significantly decreased of about $20 \%$ of total reads during acute infection, followed by a considerable increase of about $42 \%$ of total reads during the chronic phase of infection, compared to the acute infection sample (Figure 21A). Similar increased expression levels of KIR3DSO2 of about $42 \%$ (Figure 21B) between the acute and chronic phase of infection were observed in individual mm4 (LVL). Interestingly, in both individuals the relative expression levels of the inhibitory transcripts of KIR3DLO5 in $\mathrm{mm} 32$, and KIR3DLO1 in $\mathrm{mm} 4$ were increased during the acute phase of infection followed by a considerable decrease of about $18.5 \%$ or $28 \%$ of total sequencing reads, respectively. An increase of about $23.5 \%$ of total reads reflecting increased relative 
expression levels of inhibitory KIR3DLO1 transcripts in the acute phase of infection was also identified in individual mm13 (LVL) (Figure 21C), while the relative transcription level of the activating KIR3DSO1 was decreased (21\%) simultaneously. Highly increased expression levels of KIR3DSO4 of $46 \%$ to $74 \%$ of total reads already during the acute phase of infection were detected in individual mm7 (HVL), which stayed at high levels during the chronic phase of SIV infection (data not shown).

These findings might indicate that specific proliferation of NK cells expressing inhibitory KIR receptors during the acute phase of SIV infection and elevation of NK cells expressing activating KIRs during the chronic phase contribute to lower viral loads and slower disease progression. Similar expression profiles of KIR transcripts were also identified in animals of the HVL cohort but to a lesser extent. Changes in KIR gene expression are likely induced by the KIR-specific MHC class I ligands, but neither the ligand of KIR3DLO1 nor KIR3DSO2 are known in rhesus macaques. However, only few individuals showed these striking differences of expression levels of single KIRs over the course of SIV disease and have to be verified by additional individuals to confirm the influence of these individual KIRs on SIV disease outcome.
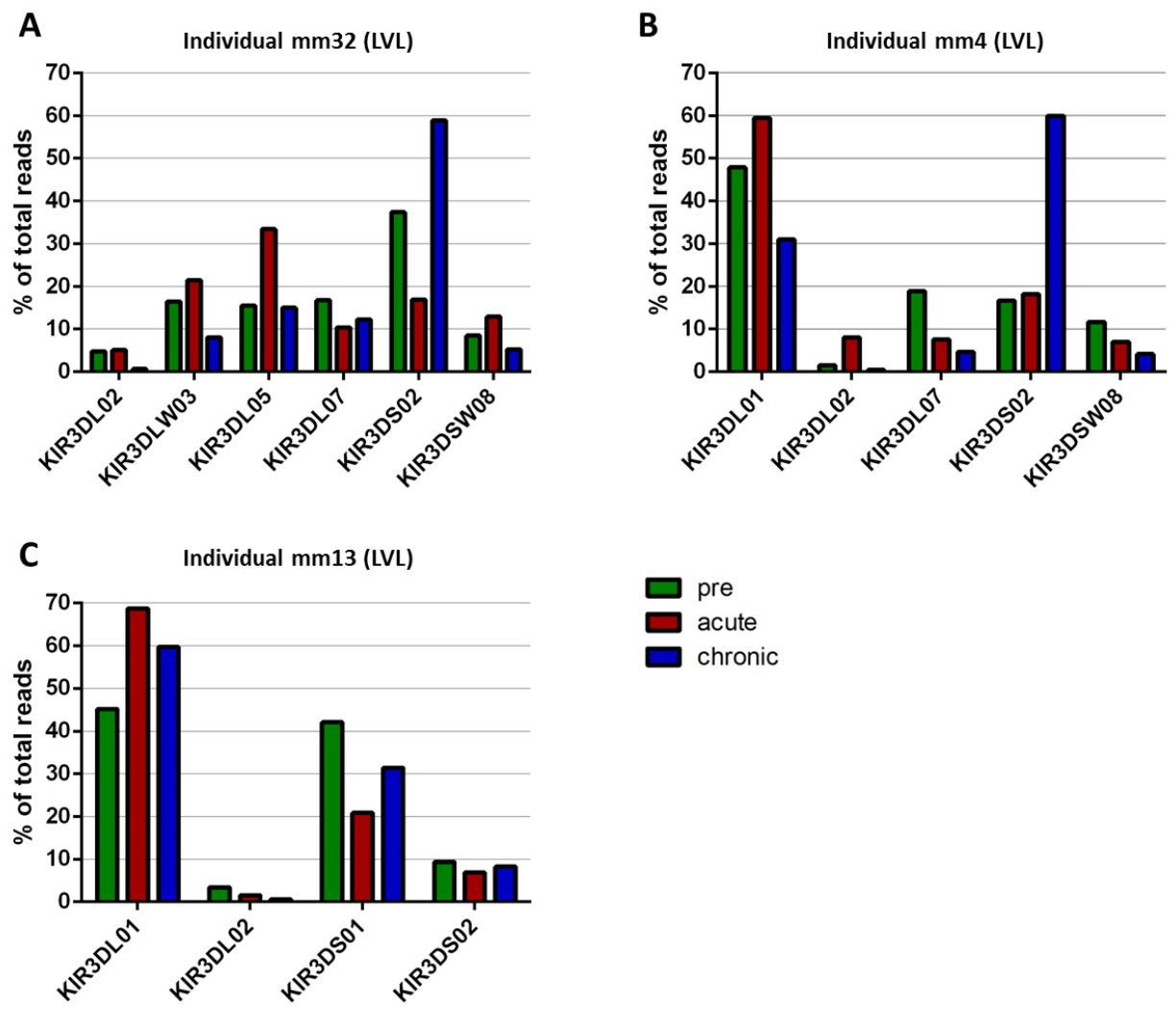

Figure 21 Comparison of three KIR transcription profiles over the course of SIV infection.

The $y$-axis indicates the percentage of total sequencing reads per individual for each KIR transcript identified. The comparison of the KIR transcripts profiles established with three different samples are shown for three individuals (A, mm32; B, mm4; C, mm13). 


\subsection{Comparison of the KIR transcript repertoire of NK cells and $\mathrm{CD}^{+} \mathrm{T}$ cells}

KIR receptors are one of the most important receptors that are expressed on NK cells and on subsets of T lymphocytes, especially $\mathrm{CD}^{+} \mathrm{T}$ cells, in humans (Phillips et al., 1995; Mingari et al., 1996; D`Andrea et al., 1998). In rhesus macaques, KIRs are expressed on the cell surface of NK cells, but also on T cells, particularly on $\mathrm{CD}^{+} \mathrm{T}$ cells, on $\gamma \delta \mathrm{T}$ cells, as well as on a minor fraction of $\mathrm{CD}^{+} \mathrm{T}$ cells (personal communication with Meike Hermes, Primate Genetics, DPZ). In this study, the KIR transcript repertoire of SIV-infected rhesus macaques was analysed from PBMC samples. To investigate potential differences between NK cells and $\mathrm{CD}^{+} \mathrm{T}$ cells, these lymphocyte subpopulations were studied separately for KIR gene transcription in uninfected rhesus macaques. The cells were separated by fluorescenceactivated cell sorting and the purified RNA was provided by Professor Aftab Ansari. Total RNA was reverse transcribed and cDNA-PCR amplicons were subjected to 454 sequencing as described before.

The content of transcribed KIR genes identified in NK cells and $\mathrm{CD} 8^{+} \mathrm{T}$ cells of each analysed rhesus macaque $(\mathrm{n}=5)$ showed no striking differences between both cell populations. In this analysis, each KIR transcript, which comprised at least $2 \%$ of total sequencing reads identified within a cell population, was detected in NK cells as well as in $\mathrm{CD}^{+} \mathrm{T}$ cells of the individual considered (Figure 22). Sequencing of PBMC samples of these animals sometimes failed to detect a few KIR transcripts, which were expressed on lower levels in the corresponding samples. However, Figure 22 shows that NK cells and $\mathrm{CD}^{+} \mathrm{T}$ cells of uninfected rhesus macaques share the identical content of KIR transcripts (individual RFn10 and RSt4), which was confirmed by samples of three additional individuals (data not shown).

The investigation of the relative expression levels of KIR transcripts in NK cells and in CD8 ${ }^{+}$ $\mathrm{T}$ cells of the identical macaque showed some differences. Relative expression levels of the activating KIR3DSO4, identified in $\mathrm{CD}^{+} \mathrm{T}$ cells were more than $20 \%$ higher compared to the transcription levels observed in NK cells in individual RFn10 (Figure 22A) and in an additional animal (data not shown), respectively. By contrast in individual RSt4 (Figure 22B) the relative transcription levels of the inhibitory KIR3DLO8 and KIR3DL1O were higher in $\mathrm{CD}^{+} \mathrm{T}$ cells (about $14 \%$ of total reads each) compared to the relative expression levels identified in NK cells. However, the small number of individuals tested, does not offer the possibility to analyse differences of the relative expression levels of $K I R$ transcripts between NK cells and $\mathrm{CD}^{+} \mathrm{T}$ cells in a comprehensive way. Similar differences of KIR expression between NK cell and T cells could only be confirmed by a maximum of two individuals and 
requires replication in additional samples. Nevertheless, the analysis indicates that NK cells and $\mathrm{CD}^{+} \mathrm{T}$ cells of an individual express the identical repertoire of KIR genes.

A

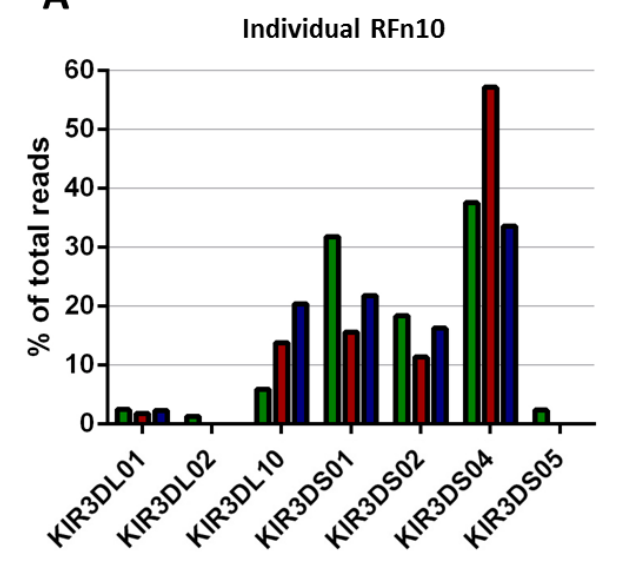

B

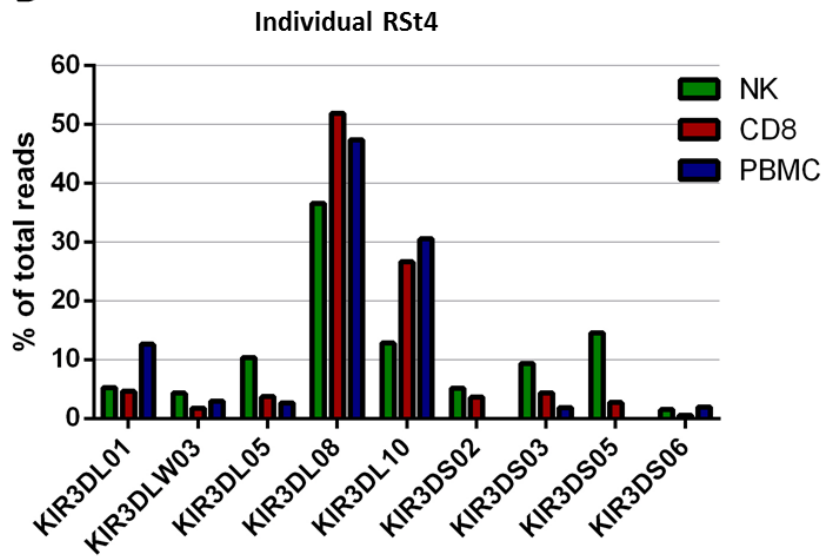

Figure 22 Comparison of $K I R$ transcription profiles of NK cells and $\mathrm{CDB}^{+} \mathrm{T}$ cells.

The distinct KIR transcripts detected are shown for both animals (A: RSt4; B: RFn10) along with the relative expression level of each KIR. The KIR typing results obtained by 454 sequencing of cDNAPCR amplicons of NK cells, CD8 ${ }^{+} \mathrm{T}$ cells and PBMCs are illustrated and the different cell subsets are indicated by different colours (legend). The comparison revealed identical KIR transcripts within the analysed cell populations of healthy individual.

\subsection{Gene expression analysis of SIV-infected rhesus macaques by real-time PCR}

During infection with HIV a multiplicity of cells of the human immune system are activated, like T and B lymphocytes, DCs, and NK cells. So far, only changes of KIR transcripts during experimental infection with SIV have been analysed in the cohort of SIV-infected rhesus macaques. A comprehensive gene expression assay was performed to identify additional changes of expression levels of 48 immune genes (2.1.7) during acute and chronic phase of SIV infection, in comparison with pre infection samples. Therefore a microfluidic-based realtime PCR approach was performed on the Biomark System. A subset of inhibitory and activating NK cell markers, like e.g. NKG2D and diverse NCRs, were analysed to get a more comprehensive overview of the mechanisms, which might contribute to the observed differences in disease progression between the HVL and LVL cohort. Additionally, markers for $\mathrm{T}$ and $\mathrm{B}$ cells as well as a panel of activation markers like $C D 223$, a lymphocyte-activation gene, which is expressed on activated T cells and NK cells, or perforin (PRF1), which is expressed by $\mathrm{NK}$ cells and $\mathrm{CD} 8^{+} \mathrm{T}$ cells, were included. The housekeeping genes GAPDH and HPRTl were used as endogenous controls. 
cDNA samples (pre, acute, chronic) of 42 SIV-infected rhesus macaques, which were divided according to plasma viral loads at set point (HVL, n=22; LVL, $n=20$ ) were analysed. The housekeeping gene $G A P D H$ was used as the reference gene and the data were used for normalisation to determine delta $\mathrm{Ct}(\Delta \mathrm{Ct})$ values. Samples prior to infection with SIV were used as the reference sample for each gene and individual to calculate $\Delta \Delta \mathrm{Ct}$ values. Relative gene expression values (fold change) of acute and chronic infection samples were determined using the $2^{-\triangle \Delta C T}$ method of Livak and Schmittgen (2001). All gene expression values (fold change) are shown in 7.3 (supplement).

Figure 23 shows the analysis of 14 selected NK cell markers compared between both cohorts during acute (A) and chronic SIV infection (B). The changes in gene expression of all NK cell markers documented, failed to be statistically significant during the acute phase of SIV infection between the HVL and LVL cohort. However, the analysis showed that the elevated expression levels of genes observed in the HVL cohort were consistently higher compared to the changes identified in the LVL cohort, except for a few individuals. While almost all NK cell markers showed increased expression levels during acute and chronic phase of infection in comparison with pre infection samples, $N K p 46$ (NCRl) mRNA levels were decreased more than 20-fold in both cohorts post infection with SIV. NKp46 is the most specific NK cell marker and reduced surface expression of NCRs (NKp46, NKp30 and NKp44) on NK cells during infection with HIV-1 in humans was already observed by De Maria et al. (2003). In the present study NKp44 (NCR2) failed to be detected by the used assay and NKp3O (NCR3) showed no significant differences over the course of SIV disease. Moreover, the analysis of the NK cell markers identified reduced mRNA levels during chronic infection compared to acute infection samples in the both cohorts. While the slightly more decreased gene expression levels during the chronic infection within the LVL cohort likely resulted from reduced numbers of activated NK cells, the reduced gene expression levels within the HVL cohort are mainly caused by a strong decline of NK cell numbers, as the animals already developed AIDS. 
A acute infection
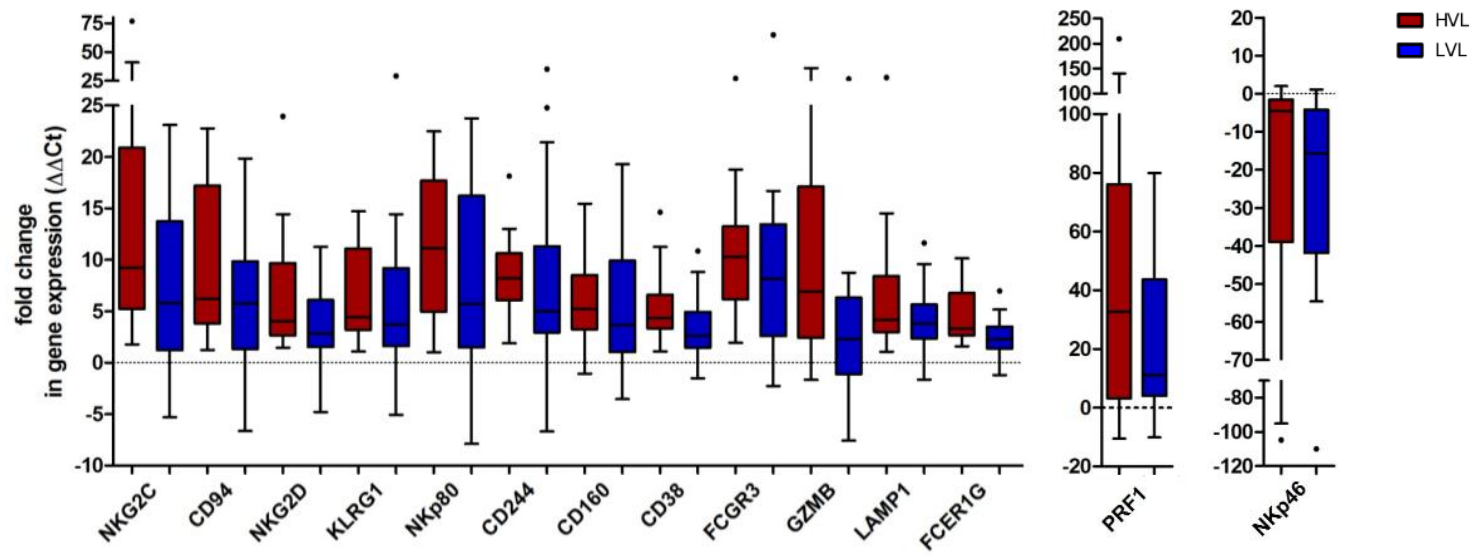

B

chronic infection
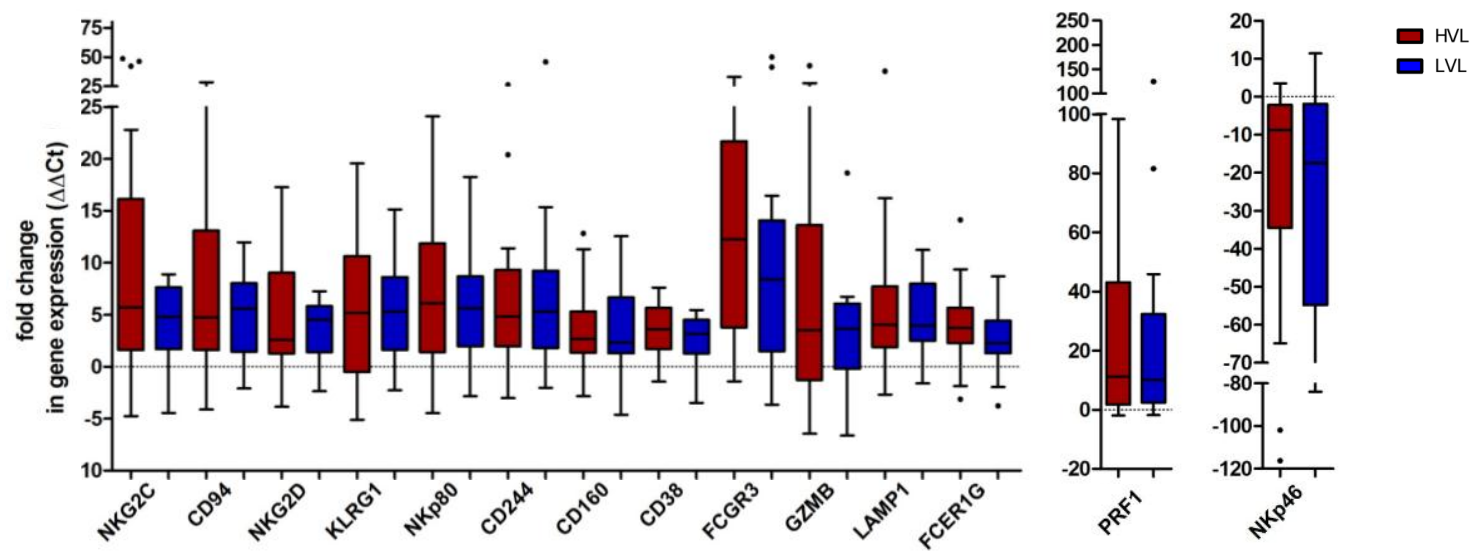

Figure 23 Gene expression analysis of NK cell markers in the HVL and LVL cohort post infection with SIV.

The box-whisker-plots (with Tukey`s correction) show the fold change of gene expression of 14 selected NK cell markers during the acute (A; HVL/LVL $n=21 / 15)$ and chronic (B; HVL/LVL $\mathrm{n}=19 / 17$ ) phase of infection compared between the HVL (red) and LVL (blue) cohort. Outliers are shown as dots. The differences were calculated with the $2^{-\triangle \Delta C T}$ method. GAPDH was used as the reference gene for normalisation ( $\Delta \mathrm{Ct}$ values) and pre infection samples were used as reference samples for each gene and individual ( $\Delta \Delta \mathrm{Ct}$ values).

Investigation of a set of activation markers of immune cells identified increased expression levels of genes involved in cell lysis such as PRF1 and granzyme B (GZMB) or indirectly indicate degranulation of PRF1 and GZMB such as LAMP1 (CD107a). These markers, which are expressed on $\mathrm{NK}$ cells and $\mathrm{CD} 8^{+} \mathrm{T}$ cells, showed considerably increased expression levels during the acute (Figure 24A) and chronic (Figure 24B) phase of SIV infection in both cohorts. About more than 25-fold reduced expression levels of $C D 70$ were observed in both cohorts post infection. CD70 is a ligand of TNF receptors and is expressed on the cell surface of activated, but not resting T and B cells. Decreased expression levels of CD70-positive cells 
were already identified in HIV-1 infected patients (Wolthers et al., 1997) and are supposed to result from impaired $\mathrm{T}$ cell help to $\mathrm{B}$ cells, leading to lower $\mathrm{B}$ cell activation and reduced CD70 expression. The mean variation of the expression levels of activation markers was very high within both cohorts, indicating interindividual differences of activation, which might be induced by plastic individual immune response to the virus.
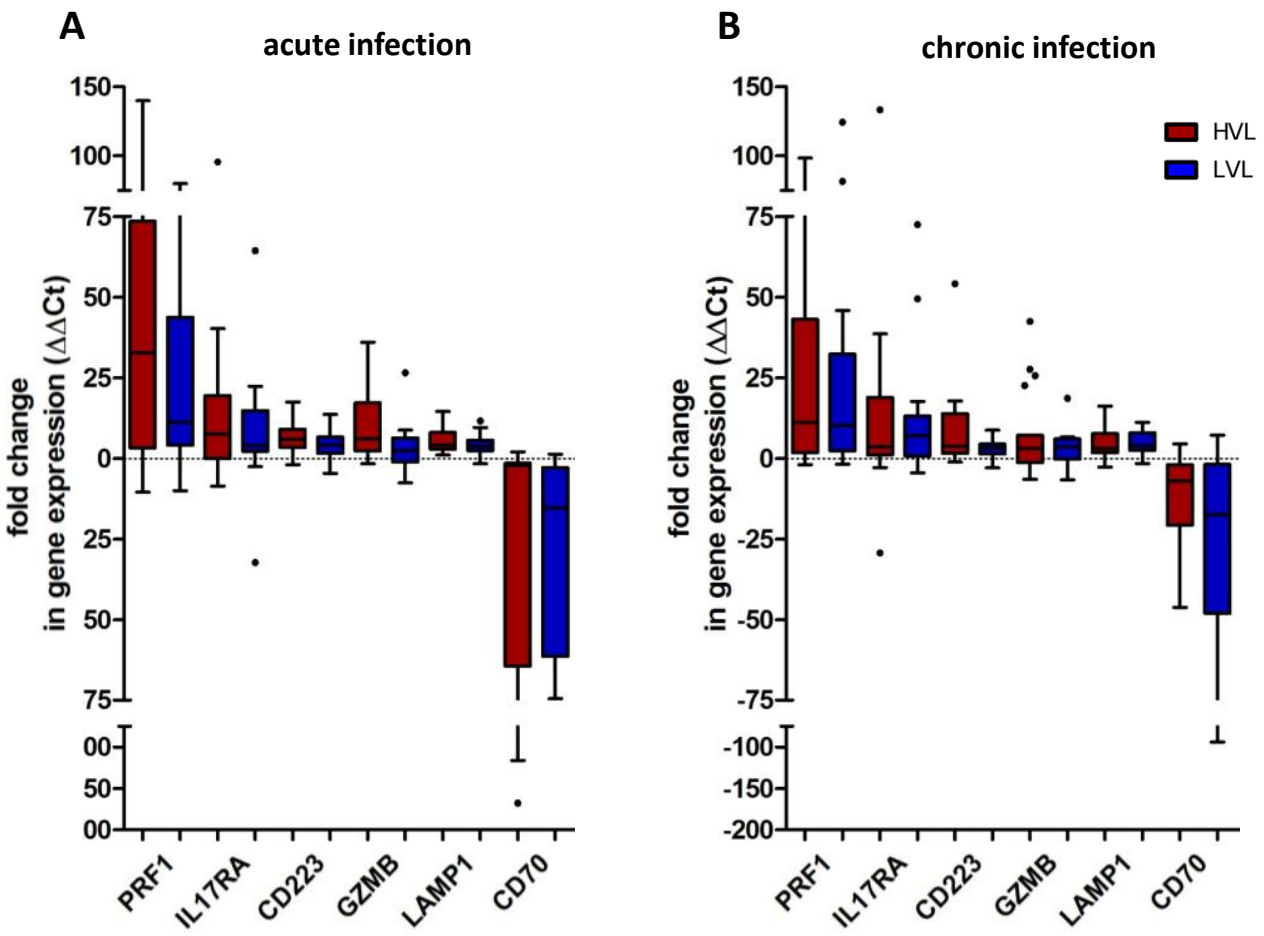

Figure 24 Gene expression analysis of activation marker in the HVL and LVL cohort post infection with SIV

The box-whisker-plots (with Tukey`s correction) show the fold change of gene expression of 6 selected activation markers during the acute $(A ; H V L / L V L n=21 / 15)$ and chronic $(B ; H V L / L V L$ $\mathrm{n}=19 / 17$ ) phase of infection compared between the HVL (red) and LVL (blue) cohort. Outliers are shown as dots. The differences were calculated with the $2^{-\triangle \Delta C T}$ method. GAPDH was used as the reference gene for normalisation $(\Delta \mathrm{Ct}$ values) and pre infection samples were used as reference samples for each gene and individual ( $\Delta \Delta \mathrm{Ct}$ values).

The expression levels of the T- and B-cell markers CD28 and CCR7 (Figure 25) were on average 3- to 4-fold reduced during acute infection and 4- to 8-fold during the chronic phase of infection in the HVL cohort, while the expression levels of these markers did not changed significantly in the LVL cohort post infection. Differences of the mRNA levels between both cohorts in the chronic phase of infection were statistically significant (CD28: $p=0.042 ; C C R 7$ : $p=0.032)$. Moreover, $C D 40 L$ (CD154), which is exclusively expressed on activated $\mathrm{CD} 4^{+} \mathrm{T}$ cells, was significantly reduced during chronic infection in the HVL cohort compared to the LVL cohort $(p=0.026)$. These findings indicate the typical loss of $\mathrm{CD}^{+} \mathrm{T}$ cells during acute 
infection and the continuous decrease during the chronic phase of SIV infection, which is more essential in animals with high viral loads. The observed changes of expression levels of the most analysed T- and B-cell markers are relatively low. However, the majority of markers are not unique for a single subset of cells and e.g. the loss of $\mathrm{CD}^{+} \mathrm{T}$ cells and the almost stable counts of $\mathrm{CD}^{+} \mathrm{T}$ cells post infection might explain the relatively weak changes of shared markers.
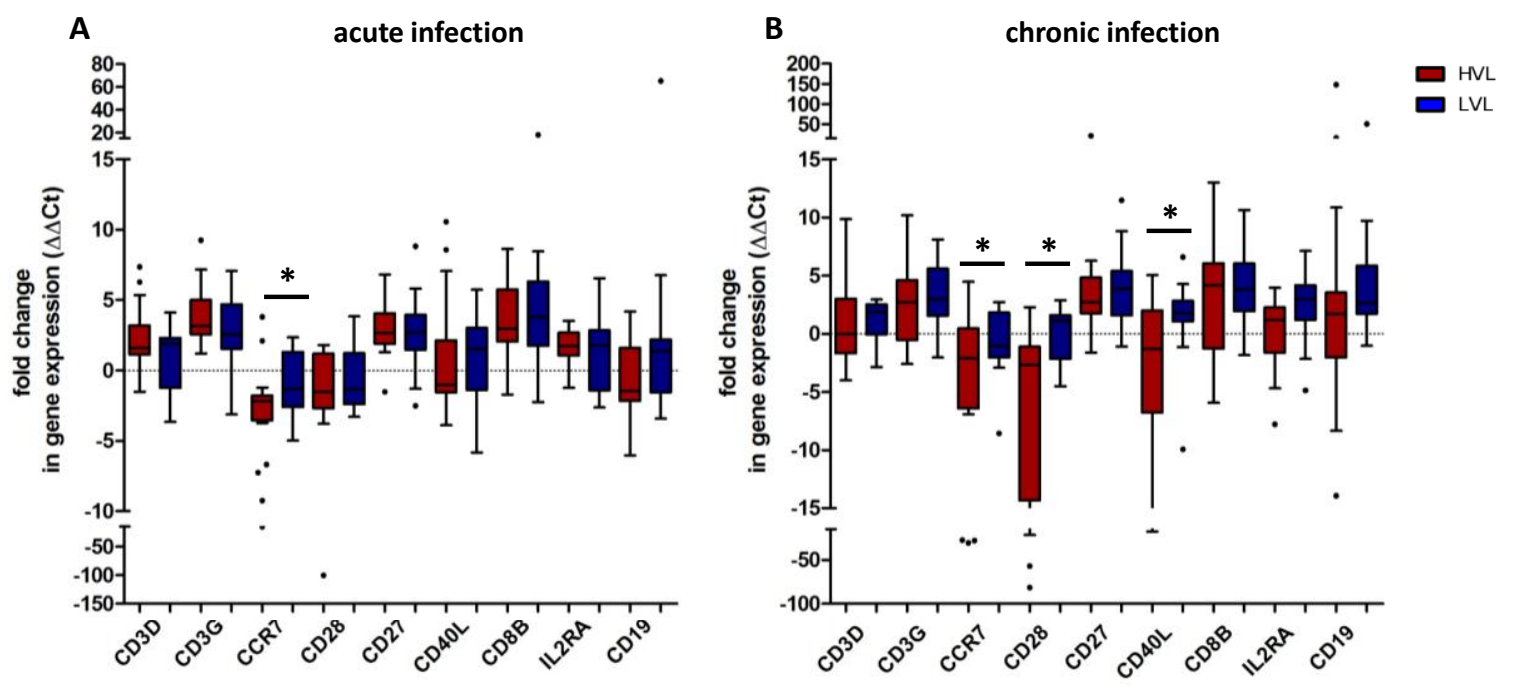

Figure 25 Gene expression analysis of T- and B-cell markers in the HVL and LVL cohort post infection with SIV

The box-whisker-plots (with Tukey`s correction) show the fold change of gene expression of 9 selected T- and B-cell markers during the acute $(\mathrm{A} ; \mathrm{HVL} / \mathrm{LVL} \mathrm{n}=21 / 15)$ and chronic $(\mathrm{B} ; \mathrm{HVL} / \mathrm{LVL}$ $\mathrm{n}=19 / 17$ ) phase of infection compared between the HVL (red) and LVL (blue) cohort. Outliers are shown as dots. The differences were calculated with the $2^{-\triangle \Delta C T}$ method. GAPDH was used as the reference gene for normalisation ( $\Delta \mathrm{Ct}$ values) and pre infection samples were used as reference samples for each gene and individual ( $\Delta \Delta \mathrm{Ct}$ values). Differences of gene expression levels between both cohorts were calculated by using two-tailed t-test. Significant differences $(p \leq 0.05)$ are marked with an asterisk $(*)$.

In summary, the data reflect the typical immune activation in response to the viral infection, like activation of NK cells and T cells. It was shown that mainly the transcription levels of activation markers of different cell subsets were strongly elevated post infection. While animals with high viral loads showed the more vigorous response to the virus, the LVL cohort showed increased expression levels of activation markers to a lesser extent. The observed differences of the most mRNA levels between both cohorts failed statistical support, except for some T- and B-cell markers that is likely due to the high variability of immune responses of individuals within the cohorts. It should be mentioned that only unsorted PBMC were used for the analysis and almost all genes in this approach are known to be expressed on different 
subsets of cells. Thereby it might happen that the changes of expression levels of specific immune genes are levelled, because they are expressed by more than one subset of cells. Elevated or reduced transcription levels of immune genes within single cell subsets might actually be much stronger than detected. However, the characteristic loss of $\mathrm{CD}^{+} \mathrm{T}$ cells during SIV infection was detected. In addition, the analysis clearly shows the strong immune activation in response to the experimental SIV infection in the rhesus macaque cohort and confirms the important role of NK cells during early infection. 


\section{Discussion}

In the last years, several studies indicated that mainly events that occur during acute HIV-1 infection and prior to effective adaptive immune responses appear to control major differences in the kinetics of viraemia, and thereby have an influence on disease progression (Haase et al., 2010). Multiple epidemiological studies in humans have demonstrated associations between KIR/MHC class I gene combinations and HIV disease progression, implicating important contributions of NK cells in defence against viral infection and viral expansion prior to initiation of adaptive response (Martin et al., 2002; Martin et al., 2007; Alter et al., 2007a). However, the functional basis for this protective immunity mediated by NK cells in viral infections in human remains largely unknown. Experimental infection of macaques with pathogenic strains of SIV serves as the primary model system for understanding HIV pathogenesis in human (Gardner and Luciw, 2008), as SIV causes an AIDS-like disease with remarkable similarities to HIV-1-mediated AIDS. Variable outcomes of SIV infection have also been observed in macaques, suggesting that there are unique aspects of virus-host interactions during the acute phase of infection. In particular Indian rhesus macaques have historically been the preferred rhesus macaque population for modelling infectious diseases. Many studies already identified MHC class I genes, which were shown to have a protective effect and are over-represented among elite controller cohorts (Loffredo et al., 2007; Yant et al., 2006). However, less is known about rhesus macaque KIR genes in SIV infection.

In this study the recently published approach of 454 sequencing of cDNA-PCR amplicons (Moreland et al., 2011) was used to establish comprehensive KIR transcription profiles of a cohort of $52 \mathrm{SIV}$-infected rhesus macaques, which was divided into two groups according to plasma viral loads at set point (HVL and LVL). This is the first time that certain "advantageous" and "disadvantageous" KIR transcripts in SIV-infected rhesus macaques were identified by using a sequence-specific KIR typing approach. Both, KIR transcripts and transcription levels of KIR genes were associated with SIV disease progression or even with spontaneous control of viral replication in elite controller animals. Additionally, samples of different time points over the course of SIV disease were analysed and changes of KIR transcription levels upon experimental SIV infection within the HVL and LVL cohorts were identified. Two inhibitory KIR genes, KIR3DL05 and KIR3DL07 showed striking changes following SIV infection in animals showing high viral replication, whereas LVL individuals showed no significant differences. 


\subsection{Diversity of rhesus macaque KIR and MHC class I transcripts}

\subsubsection{Diversity of rhesus macaque KIR gene transcription}

Human KIR haplotypes are characterised by allelic polymorphism as well as by variation in gene content (Uhrberg et al., 1997) resulting from extensive gene duplications and nonreciprocal crossing-over events (Martin et al., 2000). By now, KIR data have been reported on 109 human populations, of which 12.856 individuals were genotyped, revealing 398 different KIR genotypes (Gonzalez-Galarza et al., 2011). Even though less data have been reported on rhesus macaque $K I R$ genes, those studies pointed out that rhesus macaque KIR haplotypes are also highly polymorphic; yet even exhibit higher variability as human KIR haplotypes (Kruse et al., 2010; Blokhuis et al., 2010; Blokhuis et al., 2011).

In this study a relatively large cohort of 52 outbred rhesus macaques of Indian origin was characterised for KIR transcripts by sequencing cDNA-PCR amplicons. The comprehensive analysis revealed 19 different $K I R$ genes expressed at different frequencies within the population. While no KIR transcript could be identified in all animals, KIR3DLO1 was present in about $94 \%$ of the cohort (Figure 9). Comparable high frequencies of KIR3DLO1 were already observed in other rhesus macaque populations (Moreland et al., 2011; Kruse et al., 2010; Blokhuis et al., 2011), suggesting that KIR3DL01 represents a framework gene in rhesus macaques KIR haplotypes. KIR2DL04, KIR3DL11, KIR3DL20, and KIR3DSW08 have been proposed as framework genes in rhesus macaque KIR haplotypes (Kruse et al., 2010) but KIR3DL11 and KIR3DSW08 were present in less than $30 \%$ in the studied cohort. However, it should be mentioned that the used approach of sequencing cDNA-PCR amplicons does not select for KIR2DL04 transcripts and only transcribed KIR genes were detected, while Kruse et al. (2010) employed sequence-specific PCR from genomic DNA. Interestingly, KIR3DL20 was not detected in any macaque in this study in contrast to the study of Moreland et al. (2011). In addition to KIR3DL01, six further KIR transcripts, KIRID, KIR3DL05, KIR3DL07, KIR3DL08, KIR3DSO2, and KIR3DS05, were found at high frequencies of $\geq 50 \%$ in the cohort. Independent of the used KIR typing technique employing genomic DNA or cDNA, similar frequencies of these KIRs were determined by several studies (Moreland et al., 2011; Kruse et al., 2010; Blokhuis et al., 2011), suggesting important roles for rhesus macaque NK and T cells.

The total number of individual KIR transcripts detected in each animal of the studied rhesus macaque cohort showed considerable differences. Animals with four to eleven different 
transcribed KIR genes were identified, while the majority of animals possessed six to seven distinct $K I R$ transcripts. Only five out of 52 macaques shared the identical KIR transcript repertoire, highlighting the enormous KIR diversity of rhesus macaques and of the studied cohort. While SSP-KIR typing methods use genomic DNA and only provide information about presence and absence of KIR genes within individuals, a major advantage of the used 454 sequencing approach is that it also provides estimates of the relative expression level of $K I R$ genes. The analysis of $K I R$ transcription levels indicated highly variable expression levels of several KIRs between subjects. While some KIRs (e.g. KIRID, KIR3DLW03, KIR3DSW09) are expressed on relatively low levels ( $\leq 10 \%$ of sequence reads), the transcripts of KIR3DLO1 and KIR3DLO8 comprised 10-70\% of total sequencing reads (Figure 10). KIR expression was shown to be dominantly influenced by the complete KIR genotype and is additionally modulated by the MHC class I genotype of individuals (Shilling et al., 2002). Even though individuals share KIR transcripts, considerable differences in expression levels of these KIRs were detected.

Taken together, the established KIR transcription profiles of 52 outbred rhesus macaques studied in this work showed considerable differences in content and relative expression levels of $K I R$ transcripts, thus, indicating the high variability of the cohort and again confirming the diversity of rhesus macaque KIR haplotypes.

\subsubsection{Diversity of rhesus macaque MHC class I transcription}

While human $M H C$ class I haplotypes only contain three classical class I genes $(H L A-A,-B$ and $-C$ ), rhesus macaque $M H C$ class $I$ genes have undergone a complex series of segmental duplications such that the gene content varies substantially between macaque MHC class I haplotypes (Bontrop, 2006). An ortholog of the human HLC-C is missing in rhesus macaques, but considerable expansion within the MHC class I A and class I B gene regions contribute to MHC class $I$ haplotypes with up to 20 different Mamu-A and -B loci (Boyson et al., 1996; Daza-Vamenta et al., 2004). In this study, the recently published MHC class I typing approach of 454 sequencing of cDNA-PCR amplicons was used (O'Leary et al., 2010) to determine MHC class I profiles for each macaque of the cohort. 74 distinct MHC class I alleles were detected and individuals with eight to 15 distinct $M H C$ class I alleles were identified. Multiple characteristics have been observed previously for rhesus macaque $M H C$ class I haplotypes: (1) Each haplotype possesses at least one Maтu-AI allele (2) in combination with one or two Maтu-A2, $-A 3$, or $-A 4$ alleles (Otting et al., 2007) and (3) 
Mamu-A1 alleles and some Mати-B alleles are expressed at high transcription levels and were designated as "major" genes, while Мати-A2-A4 and several Мати-B alleles were found at lower transcription levels ("minor" genes) (Otting et al., 2005, 2007; Rosner et al., 2010). Each established MHC class I transcription profile of the analysed macaque cohort exhibit these characteristics and thereby confirms the enormous diversity of rhesus macaque MHC class I haplotypes.

\subsection{Associations of $K I R$ transcription and viral replication}

While NK cells express a large subset of different NK cell receptors, KIRs have received the most attention in terms of their role in controlling HIV-1 infection (Alter and Altfeld, 2009). KIRs are expressed on NK cells and on subsets of T cells in human (Phillips et al., 1995; Mingari et al., 1996; D`Andrea et al., 1998) as well as in rhesus macaques (personal communication with Meike Hermes; DPZ). $\mathrm{CD}^{+} \mathrm{T}$ cells play an important role in the containment of HIV-1 infection and KIR expression on $\mathrm{T}$ cells was shown to be associated with reduced TCR-mediated activation, resulting in poor antiviral activity (De Maria et al., 1997; Alter et al., 2008). To identify differences in KIR gene transcription between these cell subsets, purified NK cells and $\mathrm{CD}^{+} \mathrm{T}$ cells of uninfected rhesus macaques were analysed using 454 sequencing of cDNA-PCR amplicons. The analysis indicated that both cell populations of a healthy individual possess the identical repertoire of KIR transcripts (Figure 22 ). Based on this finding it is not possible to prove how the observed changes of KIR transcription levels upon SIV infection exclusively occurred in NK cells, $\mathrm{CD} 8^{+} \mathrm{T}$ cells, or both cell populations, because PBMC samples were used for the KIR transcription analyses reported herein. However, the expression of specific KIR transcripts will most likely inhibit or advance both $\mathrm{NK}$ cell and $\mathrm{CD} 8^{+} \mathrm{T}$ cell responses in a similar manner and thereby contribute to fast virus replication or to control the virus in a very efficient way.

For the study of KIR genes in SIV disease pathogenesis an important requirement is to identify the KIRs that are expressed by an individual. Therefore, the recently published 454 KIR typing approach of cDNA-PCR amplicons (Moreland et al., 2011) was used to perform comprehensive KIR transcription profiles of the SIV-infected rhesus macaque cohort. The cohort was divided into two groups according to plasma viral load at set point (HVL and LVL) to define KIR transcripts that are associated with viral replication in SIV-infected rhesus macaques. Moreover, some macaques with low viral replication were classified as elite controller and a small part of the HVL cohort was identified as fast progressors. Comparison 
of the established KIR transcription profiles between both cohorts detected several inhibitory KIRs that are significantly associated with viral replication and disease progression.

\subsubsection{KIR3DL transcripts are associated with viral replication}

While in human the KIR2D genes are expanded and only three KIR3D (KIR3DL1/S1, KIR3DL2, KIR3DL3) genes were identified in KIR haplotypes, the KIR3DL1/S1 alleles were found to be associated with HIV-1 disease progression and are over-represented in patients who were classified as slow disease progressors (Martin et al., 2002; Martin et al., 2007).

In contrast to human, KIR3D genes are considerably expanded in Old World monkeys (Hershberger et al., 2001). Not surprisingly, inhibitory KIR3DL genes were found to be associated with viral replication in the studied cohort of SIV-infected rhesus macaques. It was shown that transcripts of KIR3DLO2 are significantly more frequent within individuals of the LVL cohort $(p=0.022)$, while transcripts of KIR $3 D L 10$ are significantly more frequent in the high viral cohort $(p=0.022)$ (Figure 12).

Many hypotheses exist to explain the protective effect of the inhibitory KIR3DL1 allotypes in HIV-1 disease progression. The HIV protein Nef induces down-regulation of HLA-A and -B molecules, to evade destruction by virus-specific $\mathrm{CD}^{+} \mathrm{T}$ cells (Cohen et al., 1999). This down-regulation of MHC class I molecules ("missing self") could be sensed by KIR3DL1 molecules expressed on NK cells and result in killing of HIV-infected cells. HIV-derived peptides bound to the HLA molecule may also disrupt the binding of KIR3DL1 ("alteredself") and lead to killing of infected cells (Thananchai et al., 2007). A similar selective SIVinduced down-regulation of MHC class I molecules was also identified in rhesus macaques (DeGottardi et al., 2008). The ligand of rhesus macaque KIR3DL02 molecules is not known yet, and comparison of the MHC class I profiles of KIR3DLO2-positive individuals did not allow identification of a particular MHC class I allele. However, seven out of ten KIR3DLO2 ${ }^{+}$ macaques of the LVL cohort (HVL 1 of 3 ) possess Mamu-A1*001, suggesting Mamu-A1*001 as a potential ligand of KIR3DL02. Interestingly, Mamu-A1*001 is associated with long-term survival and low viral loads upon experimental SIV infection (Miller et al., 1991; Mühl et al., 2002; Zhang et al., 2002; O'Connor et al., 2003) and was also detected more frequently in the LVL cohort, but failed statistical support $(p=0.163)$. A recent study of our group already identified some KIR-MHC class I interactions between Mamu-A1*001 and different inhibitory rhesus macaque KIRs, but KIR3DL02 was not tested (Rosner et al., 2011). 
The transcripts of the inhibitory KIR3DL10 were detected more frequently in the HVL cohort ( $p=0.049)$ and only one out of six KIR3DL10-positive LVL animals belongs to the elite controller individuals. Therefore it is likely that the expression of KIR3DL10 is a putative factor contributing to the observed poor control of the virus and the decline of NK cell function during chronic infection in the HVL cohort. While the ligand of KIR3DL10 is not known, 10 out of $15 \mathrm{KIR} 3 \mathrm{DL} 10^{+}$individuals of the HVL cohort express the Mamu-Al*004 allele, which is also over-represented in this cohort $(p=0.029)$. Moreover, only two out of six $K I R 3 D L 10^{+} \mathrm{LVL}$ animals are Mamu-A1*004-positive and the KIR3DL10 ${ }^{+} \mathrm{EC}$ animal is Mamu-AI*004 negative. Мати-AI*004 was already identified in MHC class I haplotypes, which are associated with fast SIV disease progression (Sauermann et al., 2008). Combination of both KIR3DL10 and Mamu-A1*004 may promote viral replication and rapid disease progression in SIV-infected rhesus macaques by strong binding intensity, resulting in suppressed activation of KIR3DL10 ${ }^{+}$NK cells.

Although the mechanisms of KIR3DL02 and KIR3DL10 to control SIV disease progression may only be explained in context with their MHC class I ligands, these data already give information on KIRs that may contribute to control or to promote SIV viral replication. Yet, it is important to check further rhesus macaque cohorts to confirm the reported associations of KIR3DLO2 and KIR3DL1O transcripts with differential viral replication during experimental SIV infection.

\subsubsection{Higher KIR3DLO5 and KIR3DL08 transcription levels are associated with viral replication and SIV disease progression}

The used approach of 454 sequencing of cDNA-PCR amplicon also provides estimates of the relative transcription level of each KIR gene within individuals. Comparison of the expression levels of KIR genes between the HVL and LVL cohort prior to infection with SIV showed that transcripts of KIR3DLO5 are expressed at significantly higher levels in animals with high viral loads ( $p=0.028$ ), compared to the LVL cohort (Figure 14). Although higher KIR3DL08 transcription levels were observed in the LVL cohort the difference slightly failed statistical support. However, the analysis of KIR3DLO ${ }^{+}$individuals showed that higher KIR3DLO8 transcription levels are significantly associated with elite controller animals $(p=0.002)$, compared to KIR3DLO ${ }^{+}$non-EC animals (Figure 15). Moreover, among the HVL cohort some individuals were classified as fast progressors, but only four macaques within this group were $\mathrm{KIR} \mathrm{DLO} \mathrm{8}^{+}$and mainly low transcription levels (mean $10.8 \%$ of total reads) were 
detected within these individuals. These findings support the assumption that higher KIR3DL08 transcription levels are associated with spontaneous viral control.

In humans it was shown that NK cell inhibitory capacities of specific KIR3DL1 allotypes are closely linked to their abundance on NK cells and to the percentages of cells expressing these molecules within the NK cell population of an individual (Yawata et al., 2006). In addition, alleles encoding high-expression KIR3DL1 allotypes associate with slower disease progression and lower viral loads in the presence of their specific MHC class I ligands in HIV-1-infected individuals (Martin et al., 2007). Several studies implicate that specific interaction between inhibitory NK cell receptors and self-MHC during development leads to functionally competent NK cells (Anfossi et al., 2006; Kim et al., 2005), a process called "education" or "licensing". With regard to rhesus macaque KIR3DL08 molecules, this model would hypothesise that a stronger inhibition during NK cell development would lead to a more vigorous effector cell response after disruption of receptor-ligand interactions. This disruption could be caused by e.g. virus-specific down-regulation (“missing self") of MHC class I molecules (DeGottardi et al., 2008), or presentation of SIV-derived peptides ("alteredself'). KIR3DL08 transcripts were detected in comparable frequencies within the two cohorts, suggesting that the protective effect might be caused by differences of KIR3DL08 protein levels on the cell surface. A clear association of individual KIR3DLO8 alleles that are expressed at high levels was not observed (data not shown), like it was shown for alleles encoding high-expression KIR3DL1 allotypes in human (Yawata et al., 2006). This is probably due to the relatively small number of rhesus macaques tested $(n=52)$ in comparison with about 1500 studied HIV-1-infected individuals (Martin et al., 2007), but not even a trend of specific KIR3DLO8 alleles was identified. Thus, the most obvious assumption is that animals of the LVL cohort and particularly ECs possess higher percentages of KIR3DL08positive NK cells, and thereby contribute more powerful to the control of viral replication. However, the used approach does not provide information about the expression of KIR receptors on the cell surface of NK cells and rhesus macaque anti-KIR3DL08 antibodies are currently not available.

Contrasting KIR3DL08, a higher transcription level in the HVL cohort was found for the inhibitory KIR3DL05. This might be explained by strong inhibition of KIR3DL05-positive NK cells, caused by receptor-ligand interactions. In contrast to KIR3DL08, the physical binding of KIR3DL05 to Mamu-A1*001 and Mamu-A3*13 was already detected (Rosner et al., 2011). Thus, KIR3DL05 might be "educated" or "licensed" by two different types of MHC class I proteins, suggesting an important role for these KIR/MHC class I interactions in 
NK cell function similar to KIR2D/HLA-C interactions in humans. Notably, Alter et al. (2011) demonstrated that HIV-1 mutations lead to amino acid changes in HIV-1-derived peptides presented by HLA-C molecules and to increased interaction of KIR2DL2 with HLA$\mathrm{C}$ proteins presenting these mutated peptides. Thus, mutations resulting in increased binding of inhibitory KIR are obviously favoured by HIV. Interestingly, a further study reported data on high binding avidity of rhesus macaque KIR3DL05 molecules to Mamu-A1*00201 in complex with SIV-derived peptides (Colantonio et al., 2011). As higher transcription levels of KIR3DL05 were detected in HVL individuals prior to infection, KIR3DL05 ${ }^{+} \mathrm{NK}$ cells are probably strongly inhibited and do not contribute to anti-viral responses. All these findings hypothesise that KIR3DL05 molecules in complex with MHC class I ligands are important factors in determining fast SIV disease progression.

Taken together, higher expression levels of two inhibitory KIR transcripts were identified to be associated with higher viral loads (KIR3DLO5) or control of viral replication and slower disease progression (KIR3DL08). These data give first evidence of inhibitory KIRs that may contribute to the observed differences in disease outcome of SIV-infected rhesus macaques and help to select specific KIRs to identify MHC class I ligands for functional analyses.

\subsection{Phenotypic changes of immune cell marker upon experimental SIV infection}

Acute viral infections are typically characterised by rapid expansion of NK cells. The critical role of NK cells in primary viral infections has been best characterised in acute murine cytomegalovirus (MCMV) infection. Interestingly, the NK cell expansion in MCMV-infected mice is restricted to specific accumulation of Ly49 $\mathrm{H}^{+} \mathrm{NK}$ cells (Daniels et al., 2001; Dokun et al., 2001). A dramatic elevation of NK cells also occurs during primary HIV-1 infection. Mainly the cytolytic, KIR expressing CD56 ${ }^{\mathrm{dim}}$ NK cells are expanded as the first-line defence and are replaced by a functionally anergic subset of NK cell with on-going viral replication (Alter et al., 2005; Mavilio et al., 2005). Consistently, NK cell activity directly correlates with the level of viral replication during acute HIV-1 infection and declines rapidly in subjects who initiated highly active antiretroviral therapy, whereas NK cell activity remained elevated in subjects who did not initiate therapy (Alter et al., 2007b). The considerable role of NK cells in early viral control (and thereby influence the rate of disease progression) was confirmed by work in sooty mangabeys, which are natural hosts of SIV. In vivo depletion of either $\mathrm{CD} 4^{+}$or $\mathrm{CD}^{+} \mathrm{T}$ cells failed to lead to any detectable sign of disease (Barry et al., 2007; Klatt et al., 
2008) and compared to rhesus macaques higher NK cell frequencies in combination with higher levels of NK cell function were detected in sooty mangabeys (Pereira et al., 2008). Moreover, NK cell expansion following SIV infection was more rapid in natural hosts compared to infected rhesus macaques, indicating important roles of NK cells in early viral control (Ansari et al., 2011).

In the studied cohort of SIV-infected rhesus macaques a considerable expansion of NK cells was detected during acute SIV infection. While in both cohorts highly proliferating NK cells $\left(\mathrm{Ki}^{+} 7^{+} \mathrm{NK}\right.$ cells) were detected in the early phase of infection, a sharp decline of proliferating NK cells, but relatively stable NK cell numbers were observed in individuals controlling viral replication. In contrast, animals of the HVL cohort showed highly proliferating NK cells over a long period of time post infection, but with dramatically reduced NK cell numbers (data not shown; information provided by Professor Aftab Ansari).

In order to identify immune cell marker that contribute to viral control and slower disease progression or to high viral replication in combination with fast disease progression, comprehensive real-time PCR analysis were performed. The mRNA levels of diverse NK cell markers, like NKG2D, or NKp80 are elevated during acute infection within both cohorts but to a slightly greater extent in the HVL cohort. This is consistent with the common expansion of NK cells in rhesus macaques following SIV infection (Ansari et al., 2011). In addition, considerable increased mRNA levels of certain activation markers including PRF1 and GZMB were detected (Figure 24). One of the most conspicuous changes following SIV infection was observed for NKp46, which is the most specific NK cell marker (Vivier et al., 2011). In agreement with studies in human (De Maria et al., 2003; O'Connor et al., 2007) about 20-fold reduced NKp46 mRNA levels were observed in both macaque cohorts post SIV infection (Figure 23). The reduction of NCR expression levels was also confirmed by data reported on early depletion of CD56 ${ }^{\text {bright }} \mathrm{NK}$ cells in acute HIV-1 infection (Alter et al., 2005), which is the subset of NK cells that express high levels of NCRs. Interestingly, a recent study demonstrated NKp46-deficient mice to be resistant to influenza infection and to produce more INF- $\gamma$ compared to wild-type mice (Narni-Mancinelli et al., 2012). Thus, more inhibition of NKp46 transcription as observed in chronic samples of the LVL cohort might contribute to lower SIV levels in these individuals (Figure 23). The characteristic decline of $\mathrm{CD}^{+} \mathrm{T}$ cells is mirrored by decreased $C D 28, C C R 7$, and CD4OL expression levels in the chronic phase of infection, which was more significantly detected in the HVL cohort compared to the LVL cohort (Figure 25). 
In conclusion, the analysis of immune cell markers reflects the strong immune activation in response to experimental SIV infection in the rhesus macaque cohort and the typical loss of $\mathrm{CD}^{+} \mathrm{T}$ cells, mainly in individuals with high viral loads. Immune activation is nowadays regarded a better predictor of disease outcome than plasma viral load (Brenchley et al., 2010). Interestingly, a difference between progressive HIV/SIV infection (e.g. human and rhesus macaques) and nonprogressive SIV infection in natural hosts is the absence of immune activation during chronic infection. In agreement with these findings, the HVL cohort showed a stronger response to the infection, while the observed immune activation within the LVL cohort was less, mainly in the chronic phase of infection. These data indicate that the immune activation and the control of plasma viraemia might be more powerful and more specific in the early phase of infection within animals of the LVL cohort. In contrast, sustained immune activation over a long period of time post infection might contribute to immune pathology and poor control of the virus within individuals of the HVL cohort.

\subsection{Models to explain advantageous and disadvantageous KIR in SIV infection}

Several studies reported data on the phenotypic changes of NK cells following HIV infection, including the already mentioned decrease of NCRs, and increased expression of inhibitory KIR receptors (Kottilil et al., 2004; Mavilio et al., 2003).

In this study, changes of transcription levels of five distinct $K I R$ genes upon experimental SIV infection were identified (KIR3DL01, KIR3DL05, KIR3DL07, KIR3DL08, KIR3DSO2). Particularly, the transcription levels of KIR3DLO5 showed striking differences during acute SIV infection and are significantly associated with high viral replication.

A fraction of the studied rhesus macaque cohort (mm1-mm38) was already analysed for differences in KIR gene expression. Using a real-time PCR approach, it was shown that increased KIR3DL mRNA levels post infection with SIV are significantly associated with animals of the HVL cohort, while the expression of KIR3DL in the LVL cohort stayed at approximately the same level post infection (Bostik et al., 2009). In addition, two KIR3DL05 alleles were shown to be associated with high levels of SIV replication.

In this study, retrospective analyses of PBMC samples of the same rhesus macaque cohort were extended by 14 additional individuals ( $n=52)$. In agreement with Bostik et al. (2009) the relative levels of inhibitory KIR gene transcription did not change significantly over the course of experimental SIV infection in those rhesus macaques that showed low rates of virus 
replication and slow disease progression. Significantly increased transcription levels of KIR3DLO5 ( $p=0.006)$ and KIR3DL01 ( $p=0.043)$ during the acute phase of SIV infection were associated with animals showing high levels of plasma viral load (3.4.2). Particularly KIR3DL05 attracted the most attention, because two KIR3DL05 polymorphism were already identified to be associated with high viral replication within a fraction of the studied cohort (Bostik et al., 2009) and, additionally, higher KIR3DL05 expression levels prior to SIV infection were more frequently observed in the HVL cohort $(p=0.028)$. Interestingly, the higher transcription levels of KIR3DLO5 under "normal" conditions were shown to be additionally increased following SIV infection more significantly in animals of the HVL cohort $(p=0.006)$, while the differences observed in the LVL cohort clearly failed statistical support $(p=0.40)$. High binding avidity of rhesus macaque KIR3DL05 molecules to MamuA1*00201 ligands in complex with SIV-derived peptides was described by Colantonio et al. (2011), suggesting an unfavourable role of the inhibitory receptor in SIV infection. Recently, KIR-associated amino-acid polymorphisms in the HIV-1 sequence were shown to enhance binding of inhibitory KIRs to HIV-infected $\mathrm{CD} 4^{+} \mathrm{T}$ cells and thereby reduce the antiviral activity of KIR-positive NK cells (Alter et al., 2011). It is therefore hypothesised that SIV may acquire changes in peptides that stabilize interaction of KIR3DL05 to its ligand and thereby suppresses activation of KIR3DL $05^{+} \mathrm{NK}$ cells in a way that favours virus replication (Figure 26C). Given that KIR expression is fixed during NK cell development, the observed increase of KIR3DLO5 mRNA levels post infection with SIV hypothesise preferential expansion of KIR3DL05 ${ }^{+} \mathrm{NK}$ cells, like it was shown for KIR3DL1 ${ }^{+}$NK cells in early HIV-1 infection in the presence of HLA-Bw4 ligands (Alter et al., 2009). Although ligands of rhesus macaque KIR3DL05 molecules were already detected (Rosner et al., 2011; Colantonio et al., 2011), these MHC class I ligands were only found in some of the KIR3DLO5 ${ }^{+}$individuals and differences between KIR3DL05 ${ }^{+}$animals of both cohorts are ambiguous. However, two ligands (Mamu-A1, Mamu-A3) of KIR3DL05 molecules were identified by now, indicating that engagement with further MHC class I molecules are likely. These data in conjunction with the recent findings suggest that disadvantageous combinations of KIR3DLO5 and MHC class I alleles may select for changes in peptides of SIV that inhibit certain NK cell responses and thereby interfere with the host's ability to contain virus replication. To identify KIRassociated amino-acid polymorphisms in SIV, respective SI-viruses isolated from plasma samples of the macaque cohort need to be analysed for the occurrence of specific mutations. These data could provide functional evidence for the disadvantageous expression of KIR3DL05. 
While transcription levels of KIR3DL05 were significantly increased $(p=0.006)$, the expression levels of KIR3DLO7 were considerably reduced $(p=0.001)$ during acute infection. Interestingly, both KIRs were found to be frequently expressed together within individuals (21 out of 52) and the observed changes correlated inversely (Figure 19). Alignment of all known rhesus macaque KIR3DLO5 and KIR3DL07 alleles revealed $\geq 95 \%$ sequence identity. This finding might suggests that both KIRs have the potential to recognise the same MHC class I alleles and, thus, compete for binding to identical ligands. The simultaneous up-regulation of KIR3DL05 and down-regulation of KIR3DL07 could then be explained by mutations in the SIV-derived peptides leading to increased binding of KIR3DL05-expressing NK cells and to decreased binding of KIR3DL07-positive NK cells. During on-going SIV infection, this would lead to accumulation and loss of respective NK cells and to the observed changes in the corresponding KIR3DL05 and KIR3DL07 mRNA frequencies.
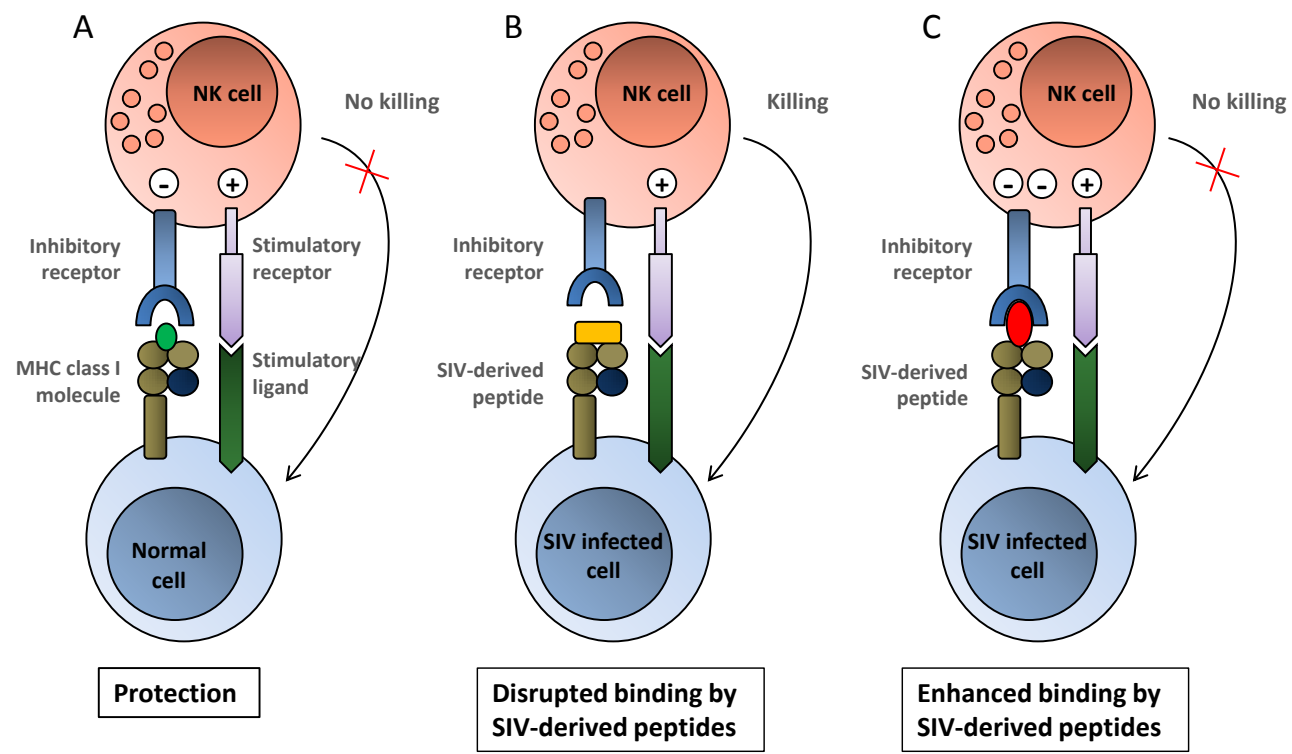

Figure 26 Advantageous and disadvantageous expression of inhibitory KIRs in SIV disease (A) Normal cells expressing host MHC class I alleles are protected from killing by NK cells via engagement of inhibitory KIR receptors expressed on the NK cell. Signals delivered by stimulatory receptors are balanced by inhibitory signal delivered by self MHC class I molecules. (B) SIV infection can cause disruption of the binding by down-regulation of MHC class I molecules or SIV-derived peptides. In this way, the NK cell does not receive inhibitory signals and therefore lyses the SIVinfected target cells ("missing-self" and "altered-self"). This might be the way how advantageous KIRs might contribute to control SIV infection. (C) KIR-associated amino-acid polymorphisms in the SIV sequence (and hence in specific SIV-derived peptides) might enhance the binding of inhibitory KIRs to SIV-infected $\mathrm{CD} 4^{+} \mathrm{T}$ cells and thereby reduce the antiviral activity of KIR-positive NK cells. This might be an explanation how disadvantageous KIRs contribute to SIV-mediated disease. 
As mentioned before higher transcription levels of KIR3DL08 are particularly associated with elite controller animals in the studied cohort. Investigation of the transcription levels of KIR3DL08 over the course of SIV infection indicated that the inhibitory KIR is expressed at constant high levels within individuals of the LVL cohort, including the ECs. In contrast, the significant lower transcription levels within the HVL cohort prior to infection were identified to be additionally reduced during chronic infection compared to pre infection samples $(p=0.030)$. Inhibitory KIR receptors can suppress NK cell activation by interaction with MHC class I ligands (Lanier, 2005; Valiante et al., 1997). Disruption of the binding, caused by MHC class I down-regulation by SIV Nef (DeGottardi et al., 2008), or presentation of antagonistic peptides (Fadda et al., 2010) results in activation of NK cells that express the inhibitory KIR and lead to killing of the target cell (Figure 26B). These findings suppose that the engagement of KIR3DL08 and MHC class I ligands is disturbed, resulting in highly activated NK cells that contribute to control viral replication, and might be a possible molecular mechanism that facilitates the protective effect of inhibitory KIRs. While the data reported herein do not provide information on surface expression of KIR3DL08 it might be that individuals of the LVL cohort and particularly elite controllers constantly possess higher percentages of KIR3DL08-positive NK cells during infection, in contrast to decreased KIR3DL08-positive NK cell numbers in HVL animals. Thus, the observed higher mRNA levels of KIR3DLO8 in elite controller animals support the assumption that these individuals control more powerful viral replication in contrast to individuals with lower or even decreased KIR3DL08 levels over the course of SIV infection.

Besides the inhibitory receptors, one activating KIR was identified showing significant differences of the expression levels over the course of SIV infection. Transcription levels of KIR3DSO2 within animals with HVL were shown to be reduced in the acute phase of infection ( $p=0.037)$ followed by increased levels of mRNA during chronic infection $(p=0.032)$. Although individuals with low viral loads showed the same trend, the differences failed statistical support. The relatively low average difference of about $3 \%$ of total sequence reads, in context with the recently observed very low binding intensity of rhesus macaque KIR3DS05 and two Mamu-A alleles (Rosner et al., 2011), suppose a weak influence of KIR3DS02 expression on SIV viral control. Interestingly, striking differences of single KIR3DS (KIR3DSO1, KIR3DSO2) transcription levels within a few individuals of the LVL cohort were observed over the course of SIV infection. However, these individuals showed no conspicuous differences in $\mathrm{CD} 4^{+} \mathrm{T}$ cell or NK cell counts (data not shown) compared to the 
other individuals of the same cohort and the observed changes occurred in single animals only and likely resulted from differences in ligand expression, which are not known yet.

In conclusion it was shown that changes of KIR3DL and KIR3DS mRNA levels post infection with SIV associate significantly with animals of the HVL cohort, while the KIR expression in animals with low viral loads stayed at approximately the same level. These findings are in agreement with the gene expression analysis of a panel of immune markers, identifying a strong immune activation over the course of SIV disease, particularly within the HVL cohort. The present KIR transcript repertoire in individuals of the LVL cohort under "normal" conditions might contribute to a fast immune response and to effective NK function for a better control of viral replication. Moreover, the repertoire of transcribed KIR genes seems to be unaffected by the virus, while the virus has a significant influence on the KIR transcription in animals within the HVL cohort, which might result in suppressed NK-cell function and thereby enabling SIV to escape the potential protective role of these KIRs. 


\section{Summary}

Multiple epidemiological studies in human demonstrated associations between $K I R / M H C$ class I gene combinations and HIV-1 disease progression, implicating important roles of KIRexpressing NK cells in defencing HIV. The nonhuman primate model of HIV is exemplified by SIV-infection of rhesus macaques. The role of KIR and MHC class I genes in SIV infection was studied in a cohort of 52 SIV-infected rhesus macaques (SIVmac239 and SIVmac251) by performing 454 sequencing of respective cDNA-PCR amplicons. The cohort was divided into high (HVL) and low (LVL) viral load according to plasma viral loads at set point.

Analysis of $M H C$ class I gene transcription confirmed previous results of Mamu-AI*004 being associated with fast SIV-disease progression.

Similar to human KIR3D genes, which are associated with HIV-1 disease progression, the analysis revealed transcribed inhibitory (KIR3DL01, KIR3DL02, KIR3DL05, KIR3DL07, KIR3DL08, KIR3DL10) and activating (KIR3DS02) rhesus macaque KIR3D genes, which are significantly associated with higher or lower viral loads.

KIR3DL05 and KIR3DL10 transcripts were associated with high viral replication, whereas KIR3DL02 and KIR3DLO8 transcripts were associated with low viral loads and elite controllers, respectively.

The KIR transcription profiles of rhesus macaques derived from sampled covering three different time points (pre, acute and chronic infection) showed that the relative transcription levels of four different inhibitory KIR genes (KIR3DL01, KIR3DL05, KIR3DL07, KIR3DL08) and one activating KIR gene (KIR3DSO2) were influenced by experimental SIV infection in the cohort of rhesus macaques. The increased expression levels of KIR3DL05 transcripts and the decreased expression levels of KIR3DLO7 transcripts in the acute phase of infection are inversely correlated in the HVL cohort.

Besides the KIR gene studies, comprehensive microfluidic-based real-time PCR analyses of 46 immune genes and cell markers reflected typical immune activation in response to SIV infection, like activation of NK cells and T cells. Animals with high viral loads showed more vigorous responses than animals of the low viral cohort. The characteristic loss of $\mathrm{CD}^{+} \mathrm{T}$ cells during SIV infection was detected in particular in the HVL cohort. 


\section{References}

Allen TM, Altfeld M, Geer SC, Kalife ET, Moore C, O'sullivan KM, Desouza I, Feeney ME, Eldridge RL, Maier EL, Kaufmann DE, Lahaie MP, Reyor L, Tanzi G, Johnston MN, Brander C, Draenert R, Rockstroh JK, Jessen H, Rosenberg ES, Mallal SA, Walker BD (2005). Selective escape from CD8+ T-cell responses represents a major driving force of human immunodeficiency virus type 1 (HIV-1) sequence diversity and reveals constraints on HIV-1 evolution. J Virol. 79(21):13239-49

Alter G, Altfeld M (2009). NK cells in HIV-1 infection: evidence for their role in the control of HIV-1 infection. J Intern Med. 265(1):29-42

Alter G, Heckerman D, Schneidewind A, Fadda L, Kadie CM, Carlson JM, Oniangue-Ndza C, Martin M, Li B, Khakoo SI, Carrington M, Allen TM, Altfeld M (2011). HIV-1 adaptation to NK-cell-mediated immune pressure. Nature. 476(7358):96-100

Alter G, Martin MP, Teigen N, Carr WH, Suscovich TJ, Schneidewind A, Streeck H, Waring M, Meier A, Brander C, Lifson JD, Allen TM, Carrington M, Altfeld M (2007a). Differential natural killer cell mediated inhibition of HIV-1 replication based on distinct KIR/HLA subtypes. J. Exp. Med. 204:3027-36

Alter G, Rihn S, Streeck H, Teigen N, Piechocka-Trocha A, Moss K, Cohen K, Meier A, Pereyra F, Walker B, Altfeld M (2008). Ligand-independent exhaustion of killer immunoglobulin-like receptor-positive CD8+ T cells in human immunodeficiency virus type 1 infection. J Virol. 82(19):9668-77

Alter G, Rihn S, Walter K, Nolting A, Martin M, Rosenberg ES, Miller JS, Carrington M, Altfeld M (2009). HLA class I subtype-dependent expansion of KIR3DS1+ and KIR3DL1+ NK cells during acute human immunodeficiency virus type 1 infection. J Virol. 83(13):6798805

Alter G, Teigen N, Ahern R, Streeck H, Meier A, Rosenberg ES, Altfeld M (2007b). Evolution of innate and adaptive effector cell functions during acute HIV-1 infection. $J$ Infect Dis. 195(10):1452-60

Alter G, Teigen N, Davis BT, Addo MM, Suscovich TJ, Waring MT, Streeck H, Johnston MN, Staller KD, Zaman MT, Yu XG, Lichterfeld M, Basgoz N, Rosenberg ES, Altfeld M (2005). Sequential deregulation of NK cell subset distribution and function starting in acute HIV-1 infection. Blood. 106(10):3366-9

Anfossi N, André P, Guia S, Falk CS, Roetynck S, Stewart CA, Breso V, Frassati C, Reviron D, Middleton D, Romagné F, Ugolini S, Vivier E (2006). Human NK cell education by inhibitory receptors for MHC class I. Immunity. 25(2):331-42

Ansari AA, Mayne AE, Takahashi Y, Pattanapanyasat K (2011). Incorporation of innate immune effector mechanisms in the formulation of a vaccine against HIV-1. Adv Exp Med Biol. 780:143-59 
Barry AP, Silvestri G, Safrit JT, Sumpter B, Kozyr N, McClure HM, Staprans SI, Feinberg MB (2007). Depletion of CD8+ cells in sooty mangabey monkeys naturally infected with simian immunodeficiency virus reveals limited role for immune control of virus replication in a natural host species. J Immunol. 178(12):8002-12

Bashirova AA, Martin MP, McVicar DW, Carrington M (2006). The killer immunoglobulinlike receptor gene cluster: tuning the genome for defense. Annu Rev Genomics Hum Genet. $7: 277-300$

Bashirova AA, Thomas R, Carrington M (2011). HLA/KIR restraint of HIV: surviving the fittest. Annu Rev Immunol. 29:295-317

Bimber BN, Moreland AJ, Wiseman RW, Hughes AL, O'Connor DH (2008). Complete characterization of killer Ig-like receptor (KIR) haplotypes in Mauritian cynomolgus macaques: novel insights into nonhuman primate KIR gene content and organization. $J$ Immunol. 181(9):6301-8

Biron CA, Nguyen KB, Pien GC, Cousens LP, Salazar-Mather TP (1999). Natural killer cells in antiviral defense: function and regulation by innate cytokines. Annu Rev Immunol. 17:189220

Blokhuis JH, Doxiadis GG, Bontrop RE (2009). A splice site mutation converts an inhibitory killer cell Ig-like receptor into an activating one. Mol Immunol. 46(4): 640-648

Blokhuis JH, van der Wiel MK, Doxiadis GG, Bontrop RE (2011). The extreme plasticity of killer cell Ig-like receptor (KIR) haplotypes differentiates rhesus macaques from humans. Eur J Immunol. 41(9):2719-28

Blokhuis JH, van der Wiel MK, Doxiadis GGM and Bontrop RE (2010). The mosaic of KIR haplotypes in rhesus macaques. Immunogenetics. 62: 295-306

Bontrop RE, Watkins DI (2005). MHC polymorphism: AIDS susceptibility in non-human primates. Trends Immunol. 26(4):227-33

Bostik P, Kobkitjaroen J, Tang W, Villinger F, Pereira LE, Little DM, Stephenson ST, Bouzyk M, Ansari AA (2009). Decreased NK cell frequency and function is associated with increased risk of KIR3DL allele polymorphism in simian immunodeficiency virus-infected rhesus macaques with high viral loads. J Immunol. 182(6):3638-49

Bostik P, Takahashi Y, Mayne AE, Ansari AA (2010). Innate immune natural killer cells and their role in HIV and SIV infection. HIV Ther. 4(4):483-504

Boyington JC, Sun PD (2002). A structural perspective on MHC class I recognition by killer cell immunoglobulin-like receptors. Mol Immunol. 38(14):1007-21

Boyle LH, Gillingham AK, Munro S, Trowsdale J (2006). Selective export of HLA-F by its cytoplasmic tail. J Immunol. 176(11):6464-72

Brenchley JM, Silvestri G, Douek DC (2010). Nonprogressive and progressive primate immunodeficiency lentivirus infections. Immunity. 32(6):737-42 
Carrington M, Martin MP, van Bergen J (2008). KIR-HLA intercourse in HIV disease. Trends Microbiol. 16(12):620-7

Carrington M, Wang S, Martin MP, Gao X, Schiffman M, Cheng J, Herrero R, Rodriguez AC, Kurman R, Mortel R, Schwartz P, Glass A, Hildesheim A (2005). Hierarchy of resistance to cervical neoplasia mediated by combinations of killer immunoglobulin-like receptor and human leukocyte antigen loci. J Exp Med. 201(7):1069-75

Cerwenka A, Lanier LL (2001). Natural killer cells, viruses and cancer. Nat Rev Immunol. $1(1): 41-9$

Chakrabarti L, Guyader M, Alizon M, Daniel MD, Desrosiers RC, Tiollais P, Sonigo P (1987). Sequence of simian immunodeficiency virus from macaque and its relationship to other human and simian retroviruses. Nature. 328(6130):543-7

Cohen GB, Gandhi RT, Davis DM, Mandelboim O, Chen BK, Strominger JL, Baltimore D (1999). The selective downregulation of class I major histocompatibility complex proteins by HIV-1 protects HIV-infected cells from NK cells. Immunity. 10(6):661-71

Colantonio AD, Bimber BN, Neidermyer WJ Jr, Reeves RK, Alter G, Altfeld M, Johnson RP, Carrington M, O'Connor DH, Evans DT (2011). KIR polymorphisms modulate peptidedependent binding to an MHC class I ligand with a Bw6 motif. PLoS Pathog. 7(3):e1001316

Cooper MA, Fehniger TA, Caligiuri MA (2001). The biology of human natural killer-cell subsets. Trends Immunol. 22(11):633-40

D'Andrea A, Lanier LL (1998). Killer cell inhibitory receptor expression by T cells. Curr Top Microbiol Immunol. 230:25-39

Daniels KA, Devora G, Lai WC, O'Donnell CL, Bennett M, Welsh RM (2001). Murine cytomegalovirus is regulated by a discrete subset of natural killer cells reactive with monoclonal antibody to Ly49H. J Exp Med. 194(1):29-44

Daza-Vamenta R, Glusman G, Rowen L, Guthrie B, Geraghty DE (2004). Genetic divergence of the rhesus macaque major histocompatibility complex. Genome Res. 14(8):1501-15

De Maria A, Ferraris A, Guastella M, Pilia S, Cantoni C, Polero L, Mingari MC, Bassetti D, Fauci AS, Moretta L (1997). Expression of HLA class I-specific inhibitory natural killer cell receptors in HIV-specific cytolytic T lymphocytes: impairment of specific cytolytic functions. Proc Natl Acad Sci U S A. 94(19):10285-8

De Maria A, Fogli M, Costa P, Murdaca G, Puppo F, Mavilio D, Moretta A, Moretta L (2003). The impaired NK cell cytolytic function in viremic HIV-1 infection is associated with a reduced surface expression of natural cytotoxicity receptors (NKp46, NKp30 and NKp44). Eur J Immunol. 33:2410-2418

DeGottardi MQ, Specht A, Metcalf B, Kaur A, Kirchhoff F, Evans DT (2008). Selective downregulation of rhesus macaque and sooty mangabey major histocompatibility complex class I molecules by Nef alleles of simian immunodeficiency virus and human immunodeficiency virus type 2. J Virol. 82(6):3139-46 
Dokun AO, Kim S, Smith HR, Kang HS, Chu DT, Yokoyama WM (2001). Specific and nonspecific NK cell activation during virus infection. Nat Immunol. 2(10):951-6

Fadda L, Borhis G, Ahmed P, Cheent K, Pageon SV, Cazaly A, Stathopoulos S, Middleton D, Mulder A, Claas FH, Elliott T, Davis DM, Purbhoo MA, Khakoo SI (2010). Peptide antagonism as a mechanism for NK cell activation. Proc Natl Acad Sci U S A. 107(22):1016010165

Fellay J, Shianna KV, Ge D, Colombo S, Ledergerber B, Weale M, Zhang K, Gumbs C, Castagna A, Cossarizza A, Cozzi-Lepri A, De Luca A, Easterbrook P, Francioli P, Mallal S, Martinez-Picado J, Miro JM, Obel N, Smith JP, Wyniger J, Descombes P, Antonarakis SE, Letvin NL, McMichael AJ, Haynes BF, Telenti A, Goldstein DB (2007). A whole-genome association study of major determinants for host control of HIV-1. Science. 317(5840):944-7

Fernandez NC, Lozier A, Flament C, Ricciardi-Castagnoli P, Bellet D, Suter M, Perricaudet M, Tursz T, Maraskovsky E, Zitvogel L (1999). Dendritic cells directly trigger NK cell functions: cross-talk relevant in innate anti-tumor immune responses in vivo. Nat Med. 5(4):405-11

Fiebig EW, Wright DJ, Rawal BD, Garrett PE, Schumacher RT, Peddada L, Heldebrant C, Smith R, Conrad A, Kleinman SH, Busch MP (2003). Dynamics of HIV viremia and antibody seroconversion in plasma donors: implications for diagnosis and staging of primary HIV infection. AIDS. 17(13):1871-9

Franchini G, Gurgo C, Guo HG, Gallo RC, Collalti E, Fargnoli KA, Hall LF, Wong-Staal F, Reitz MS Jr (1987). Sequence of simian immunodeficiency virus and its relationship to the human immunodeficiency viruses. Nature. 328(6130):539-43

Freud AG, Caligiuri MA (2006). Human natural killer cell development. Immunol Rev. 214:56-72

Garcia-Lora A, Algarra I, Garrido F (2003). MHC class I antigens, immune surveillance, and tumor immune escape. J Cell Physiol. 195(3):346-355

Gardner MB, Luciw PA (2008). Macaque models of human infectious disease. ILAR J. 49(2):220-55

Gasser S, Orsulic S, Brown EJ, Raulet DH (2005). The DNA damage pathway regulates innate immune system ligands of the NKG2D receptor. Nature. 436(7054):1186-90

Gonzalez-Galarza FF, Christmas S, Middleton D, Jones AR (2011). Allele frequency net: a database and online repository for immune gene frequencies in worldwide populations. Nucleic Acids Res. 39:D913-9

Guethlein LA, Flodin LR, Adams EJ, Parham P (2002). NK cell receptors of the orangutan (Pongo pygmaeus): a pivotal species for tracking the coevolution of killer cell Ig-like receptors with MHC-C. J Immunol. 169(1):220-9

Guethlein LA, Older Aguilar AM, Abi-Rached L, Parham P (2007). Evolution of killer cell Ig-like receptor (KIR) genes: definition of an orangutan KIR haplotype reveals expansion of lineage III KIR associated with the emergence of MHC-C. J Immunol. 179(1):491-504 
Gumperz JE, Litwin V, Phillips JH, Lanier LL, Parham P (1995). The Bw4 public epitope of HLA-B molecules confers reactivity with natural killer cell clones that express NKB1, a putative HLA receptor. J Exp Med. 181(3):1133-44

Hahn BH, Shaw GM, De Cock KM, Sharp PM (2000). AIDS as a zoonosis: Scientific and public health implications. Science. 287, 607-614

Herberman RB, Nunn ME, Holden HT, Lavrin DH (1975). Natural cytotoxic reactivity of mouse lymphoid cells against syngeneic and allogeneic tumors. II. Characterization of effector cells. Int J Cancer. 16(2):230-9

Hershberger KL, Kurian J, Korber BT, Letvin NL (2005). Killer cell immunoglobulin-like receptors (KIR) of the African-origin sabaeus monkey: evidence for recombination events in the evolution of KIR. Eur J Immunol. 35(3):922-35

Hershberger KL, Shyam R, Miura A, Letvin NL (2001). Diversity of the killer cell Ig-like receptors of rhesus monkeys. J Immunol. 166(7):4380-90

Hsu KC, Liu XR, Selvakumar A, Mickelson E, O'Reilly RJ, Dupont B (2002). Killer Ig-like receptor haplotype analysis by gene content: evidence for genomic diversity with a minimum of six basic framework haplotypes, each with multiple subsets. J Immunol. 169(9): 51185129.

Huber I, Walter L, Wimmer R, Pasantes JJ, Günther E, Schempp W (2003). Cytogenetic mapping and orientation of the rhesus macaque MHC. Cytogenet Genome Res. 103(1-2):144149

Ishitani A, Sageshima N, Lee N, Dorofeeva N, Hatake K, Marquardt H, Geraghty DE (2003). Protein expression and peptide binding suggest unique and interacting functional roles for HLA-E, F, and G in maternal-placental immune recognition. J Immunol. 171(3):1376-84

Karlhofer FM, Ribaudo RK, Yokoyama WM (1992). MHC class I alloantigen specificity of Ly-49+ IL-2-activated natural killer cells. Nature. 358(6381):66-70

Kärre K, Ljunggren HG, Piontek G, Kiessling R (1986). Selective rejection of H-2-deficient lymphoma variants suggests alternative immune defence strategy. Nature. 319(6055):675-8

Kelley J, Walter L, Trowsdale J (2005).Comparative genomics of natural killer cell receptor gene clusters. PLoS Genet. 1(2):129-39

Khakoo SI, Rajalingam R, Shum BP, Weidenbach K, Flodin L, Muir DG, Canavez F, Cooper SL, Valiante NM, Lanier LL, Parham P (2000). Rapid evolution of NK cell receptor systems demonstrated by comparison of chimpanzees and humans. Immunity. 12(6):687-98

Khakoo SI, Thio CL, Martin MP, Brooks CR, Gao X, Astemborski J, Cheng J, Goedert JJ, Vlahov D, Hilgartner M, Cox S, Little AM, Alexander GJ, Cramp ME, O'Brien SJ, Rosenberg WM, Thomas DL, Carrington M (2004). HLA and NK cell inhibitory receptor genes in resolving hepatitis C virus infection. Science. 305(5685):872-4 
Kiessling R, Klein E, Pross H, Wigzell H (1975). "Natural" killer cells in the mouse. II. Cytotoxic cells with specificity for mouse Moloney leukemia cells. Characteristics of the killer cell. Eur J Immunol. 5(2):117-21

Kim S, Poursine-Laurent J, Truscott SM, Lybarger L, Song YJ, Yang L, French AR, Sunwoo JB, Lemieux S, Hansen TH, Yokoyama WM (2005). Licensing of natural killer cells by host major histocompatibility complex class I molecules. Nature. 436(7051):709-13

Klatt NR, Villinger F, Bostik P, Gordon SN, Pereira L, Engram JC, Mayne A, Dunham RM, Lawson B, Ratcliffe SJ, Sodora DL, Else J, Reimann K, Staprans SI, Haase AT, Estes JD, Silvestri G, Ansari AA (2008). Availability of activated CD4+ T cells dictates the level of viremia in naturally SIV-infected sooty mangabeys. J Clin Invest. 118(6):2039-49

Kottilil S, Shin K, Planta M, McLaughlin M, Hallahan CW, Ghany M, Chun TW, Sneller MC, Fauci AS (2004). Expression of chemokine and inhibitory receptors on natural killer cells: effect of immune activation and HIV viremia. J Infect Dis. 189(7):1193-8

Kruse PH, Rosner C, Walter L (2010). Characterization of rhesus macaque KIR genotypes and haplotypes. Immunogenetics. 62:281-293

Kulski JK, Anzai T, Shiina T, Inoko H (2004). Rhesus macaque class I duplicon structures, organization, and evolution within the alpha block of the major histocompatibility complex. Mol Biol Evol. 21(11):2079-91

Lanier LL (1998). NK cell receptors. Annu Rev Immunol. 16:359-93

Lanier LL (2005). NK cell recognition. Annu Rev Immunol. 23:225-74

Lanier LL, Le AM, Civin CI, Loken MR, Phillips JH (1986). The relationship of CD16 (Leu11) and Leu-19 (NKH-1) antigen expression on human peripheral blood NK cells and cytotoxic T lymphocytes. J Immunol. 136(12):4480-6

Livak KJ, Schmittgen TD (2001). Analysis of relative gene expression data using real-time quantitative PCR and the 2(-Delta Delta C(T)) Method. Methods. 25:402-408

Ljunggren HG, Kärre K (1990). In search of the 'missing self': MHC molecules and NK cell recognition. Immunol Today. 11(7):237-44

Loffredo JT, Maxwell J, Qi Y, Glidden CE, Borchardt GJ, Soma T, Bean AT, Beal DR, Wilson NA, Rehrauer WM, Lifson JD, Carrington M, Watkins DI (2007). Mamu-B*08positive macaques control simian immunodeficiency virus replication. J Virol. 81(16):88278832

Long EO (1999). Regulation of immune responses through inhibitory receptors. Annu Rev Immunol. 17:875-904

Mailliard RB, Son YI, Redlinger R, Coates PT, Giermasz A, Morel PA, Storkus WJ, Kalinski P (2003). Dendritic cells mediate NK cell help for Th1 and CTL responses: twosignal requirement for the induction of NK cell helper function. J Immunol. 171(5):2366-73 
Mandelboim O, Reyburn HT, Valés-Gómez M, Pazmany L, Colonna M, Borsellino G, Strominger JL (1996). Protection from lysis by natural killer cells of group 1 and 2 specificity is mediated by residue 80 in human histocompatibility leukocyte antigen $\mathrm{C}$ alleles and also occurs with empty major histocompatibility complex molecules. J Exp Med. 184(3):913-22

Marsh SG, Parham P, Dupont B, Geraghty DE, Trowsdale J, Middleton D, Vilches C, Carrington M, Witt C, Guethlein LA, Shilling H, Garcia CA, Hsu KC, Wain H (2002). Killercell immunoglobulin-like receptor (KIR) nomenclature report, 2002. Hum Immunol. 64(6):648-54

Martin AM, Freitas EM, Witt CS, Christiansen FT (2000). The genomic organization and evolution of the natural killer immunoglobulin-like receptor (KIR) gene cluster. Immunogenetics. 51(4-5):268-80

Martin MP, Gao X, Lee JH, Nelson GW, Detels R, Goedert JJ, Buchbinder S, Hoots K, Vlahov D, Trowsdale J, Wilson M, O'Brien SJ, Carrington M (2002). Epistatic interaction between KIR3DS1 and HLA-B delays the progression to AIDS. Nat. Genet. 31:429-34

Martin MP, Qi Y, Gao X, Yamada E, Martin JN, Pereyra F, Colombo S, Brown EE, Shupert WL, Phair J, Goedert JJ, Buchbinder S, Kirk GD, Telenti A, Connors M, O'Brien SJ, Walker BD, Parham P, Deeks SG, McVicar DW, Carrington M (2007). Innate partnership of HLA-B and KIR3DL1 subtypes against HIV-1. Nat Genet. 39(6):733-40

Mavilio D, Benjamin J, Daucher M, Lombardo G, Kottilil S, Planta MA, Marcenaro E, Bottino C, Moretta L, Moretta A, Fauci AS (2003). Natural killer cells in HIV-1 infection: dichotomous effects of viremia on inhibitory and activating receptors and their functional correlates. Proc Natl Acad Sci U S A. 100(25):15011-6

Mavilio D, Lombardo G, Benjamin J, Kim D, Follman D, Marcenaro E, O'Shea MA, Kinter A, Kovacs C, Moretta A, Fauci AS (2005). Characterization of CD56-/CD16+ natural killer (NK) cells: a highly dysfunctional NK subset expanded in HIV-infected viremic individuals. Proc Natl Acad Sci U S A. 102(8):2886-91

McClure HM, Anderson DC, Fultz PN, Ansari AA, Lockwood E, Brodie A (1989). Spectrum of disease in macaque monkeys chronically infected with SIV/SMM. Vet Immunol Immunopathol. 21(1):13-24

Medzhitov R, Janeway CA Jr (1998). Innate immune recognition and control of adaptive immune responses. Semin Immunol. 10(5):351-3

Middleton D, Gonzelez F (2010). The extensive polymorphism of KIR genes. Immunology. 129(1): 8-19

Migueles SA, Sabbaghian MS, Shupert WL, Bettinotti MP, Marincola FM, Martino L, Hallahan CW, Selig SM, Schwartz D, Sullivan J, Connors M (2000). HLA B*5701 is highly associated with restriction of virus replication in a subgroup of HIV-infected long term nonprogressors. Proc Natl Acad Sci U S A. 97(6):2709-14

Miller MD, Yamamoto H, Hughes AL, Watkins DI, Letvin NL (1991). Definition of an epitope and MHC class I molecule recognized by gag-specific cytotoxic T lymphocytes in SIVmac-infected rhesus monkeys. J Immunol. 147(1): 320-329 
Mingari MC, Schiavetti F, Ponte M, Vitale C, Maggi E, Romagnani S, Demarest J, Pantaleo G, Fauci AS, Moretta L (1996). Human CD8+ T lymphocyte subsets that express HLA class I-specific inhibitory receptors represent oligoclonally or monoclonally expanded cell populations. Pro. Natl Acad. Sci USA. 93, 12433 \pm 12438

Moffett-King A (2002). Natural killer cells and pregnancy. Nat. Rev. Immunol. 2:656-63

Moreland AJ, Guethlein LA, Reeves RK, Broman KW, Johnson RP, Parham P, O'Connor DH, Bimber BN (2011). Characterization of killer immunoglobulin-like receptor genetics and comprehensive genotyping by pyrosequencing in rhesus macaques. BMC Genomics. 12:295

Moretta A, Bottino C, Vitale M, Pende D, Cantoni C, Mingari MC, Biassoni R, Moretta L (2001). Activating receptors and coreceptors involved in human natural killer cell-mediated cytolysis. Annu Rev Immunol. 19:197-223

Mühl T, Krawczak M, Ten Haaft P, Hunsmann G, Sauermann U (2002). MHC class I alleles influence set-point viral load and survival time in simian immunodeficiency virus-infected rhesus monkeys. J Immunol. 169: 3438-3446

Narni-Mancinelli E, Jaeger BN, Bernat C, Fenis A, Kung S, De Gassart A, Mahmood S, Gut M, Heath SC, Estellé J, Bertosio E, Vely F, Gastinel LN, Beutler B, Malissen B, Malissen M, Gut IG, Vivier E, Ugolini S (2012).Tuning of natural killer cell reactivity by NKp46 and Helios calibrates T cell responses. Science. 335(6066):344-8

Natarajan K, Dimasi N, Wang J, Mariuzza RA, Margulies DH (2002). Structure and function of natural killer cell receptors: multiple molecular solutions to self, nonself discrimination. Annu Rev Immunol. 20:853-85

O'Connor DH, Mothe BR, Weinfurter JT, Fuenger S, Rehrauer WM, Jing P, Rudersdorf RR, Liebl ME, Krebs K, Vasquez J, Dodds E, Loffredo J, Martin S, McDermott AB, Allen TM, Wang C, Doxiadis GG, Montefiori DC, Hughes A, Burton DR, Allison DB, Wolinsky SM, Bontrop R, Picker LJ, Watkins DI (2003). Major histocompatibility complex class I alleles associated with slow simian immunodeficiency virus disease progression bind epitopes recognized by dominant acute-phase cytotoxic-T-lymphocyte responses. J Virol. 77: 90299040

O'Connor GM, Holmes A, Mulcahy F, Gardiner CM (2007). Natural Killer cells from longterm non-progressor HIV patients are characterized by altered phenotype and function. Clin Immunol. 124(3):277-83

O'Leary CE, Wiseman RW, Karl JA, Bimber BN, Lank SM, Tuscher JJ, O'Connor DH (2009). Identification of novel MHC class I sequences in pig-tailed macaques by amplicon pyrosequencing and full-length cDNA cloning and sequencing. Immunogenetics. 61(10):689701

Orange JS, Fassett MS, Koopman LA, Boyson JE, Strominger JL (2002). Viral evasion of natural killer cells. Nat Immunol. 3(11):1006-1012

Otting N, de Vos-Rouweler AJ, Heijmans CM, de Groot NG, Doxiadis GG, Bontrop RE (2007). MHC class I A region diversity and polymorphism in macaque species. Immunogenetics. 59(5):367-75 
Otting N, Heijmans CM, Noort RC, de Groot NG, Doxiadis GG, van Rood JJ, Watkins DI, Bontrop RE (2005). Unparalleled complexity of the MHC class I region in rhesus macaques. Proc Natl Acad Sci USA. 102:1626-1631

Otting N, Heijmans CM, van der Wiel M, de Groot NG, Doxiadis GG, Bontrop RE (2008). A snapshot of the Mamu-B genes and their allelic repertoire in rhesus macaques of Chinese origin. Immunogenetics. 60(9):507-14

Parham P, Abi-Rached L, Matevosyan L, Moesta AK, Norman PJ, Older Aguilar AM, Guethlein LA (2010). Primate-specific regulation of natural killer cells. J Med Primatol. 39(4):194-212

Pereira LE, Johnson RP, Ansari AA (2008). Sooty mangabeys and rhesus macaques exhibit significant divergent natural killer cell responses during both acute and chronic phases of SIV infection. Cell Immunol. 254(1):10-9

Pessino A, Sivori S, Bottino C, Malaspina A, Morelli L, Moretta L, Biassoni R, Moretta A (1998). Molecular cloning of NKp46: a novel member of the immunoglobulin superfamily involved in triggering of natural cytotoxicity. J Exp Med. 188(5):953-60

Phillips JH, Gumperz JE, Parham P, Lanier LL (1995). Superantigen-dependent, cellmediated cytotoxicity inhibited by MHC class I receptors on T lymphocytes. Science. 268, 403-405.

Rajalingam R, Parham P, Abi-Rached L (2004). Domain shuffling has been the main mechanism forming new hominoid killer cell Ig-like receptors. J Immunol. 172(1):356-69

Raulet DH, Vance RE (2006). Self-tolerance of natural killer cells. Nat Rev Immunol. 6(7):520-31

Robinson J, Waller MJ, Parham P, de Groot N, Bontrop R, Kennedy LJ, Stoehr P, Marsh SG (2003). IMGT/HLA and IMGT/MHC: sequence databases for the study of the major histocompatibility complex. Nucleic Acids Res. 31(1):311-4

Rosner C, Kruse PH, Hermes M, Otto N, Walter L (2011). Rhesus macaque inhibitory and activating KIR3D interact with Mamu-A-encoded ligands. J Immunol. 186(4):2156-63

Rosner C, Kruse PH, Lübke T, Walter L (2010). Erratum to: rhesus macaque MHC class I molecules show differential subcellular localizations. Immunogenetics. 62(6):409-18

Sambrook JG, Bashirova A, Palmer S, Sims S, Trowsdale J, Abi-Rached L, Parham P, Carrington M, Beck S (2005). Single haplotype analysis demonstrates rapid evolution of the killer immunoglobulin-like receptor (KIR) loci in primates. Genome Res. 15(1):25-35

Santourlidis S, Trompeter HI, Weinhold S, Eisermann B, Meyer KL, Wernet P, Uhrberg M (2002). Crucial role of DNA methylation in determination of clonally distributed killer cell Ig-like receptor expression patterns in NK cells. J Immunol. 169(8):4253-61 
Sauermann U, Siddiqui R, Suh YS, Platzer M, Leuchte N, Meyer H, Mätz-Rensing K, Stoiber H, Nürnberg P, Hunsmann G, Stahl-Hennig C, Krawczak M (2008). Mhc class I haplotypes associated with survival time in simian immunodeficiency virus (SIV)-infected rhesus macaques. Genes Immun. 9(1):69-80

Selvakumar A, Steffens U, Palanisamy N, Chaganti RS, Dupont B (1997). Genomic organization and allelic polymorphism of the human killer cell inhibitory receptor gene KIR103. Tissue Antigens. 49(6):564-73

Shilling HG, Guethlein LA, Cheng NW, Gardiner CM, Rodriguez R, Tyan D, Parham P (2002a). Allelic polymorphism synergizes with variable gene content to individualize human KIR genotype. J Immunol. 168(5):2307-15

Shilling HG, Young N, Guethlein LA, Cheng NW, Gardiner CM, Tyan D, Parham P: (2002b) Genetic control of human NK cell repertoire. J Immunol. 169(1):239-247

Stebbins CC, Watzl C, Billadeau DD, Leibson PJ, Burshtyn DN, Long EO (2003). Vav1 dephosphorylation by the tyrosine phosphatase SHP-1 as a mechanism for inhibition of cellular cytotoxicity. Mol Cell Biol. 23(17):6291-9

Stulberg MJ, Wright PW, Dang H, Hanson RJ, Miller JS, Anderson SK (2007). Identification of distal KIR promoters and transcripts. Genes Immun. 8(2):124-30

Thananchai H, Gillespie G, Martin MP, Bashirova A, Yawata N, Yawata M, Easterbrook P, McVicar DW, Maenaka K, Parham P, Carrington M, Dong T, Rowland-Jones S (2007). Cutting Edge: Allele-specific and peptide-dependent interactions between KIR3DL1 and HLA-A and HLA-B. J Immunol. 178(1):33-7

The MHC sequencing consortium (1999). Complete sequence and gene map of a human major histocompatibility complex. Nature. 401(6756):921-3

Trowsdale J, Barten R, Haude A, Stewart CA, Beck S, Wilson MJ (2001). The genomic context of natural killer receptor extended gene families. Immunol Rev. 181:20-38

Uhrberg M, Parham P, Wernet P (2002). Definition of gene content for nine common group B haplotypes of the Caucasoid population: KIR haplotypes contain between seven and eleven KIR genes. Immunogenetics. 54(4): 221-229

Uhrberg M, Valiante NM, Shum BP, Shilling HG, Lienert-Weidenbach K, Corliss B, Tyan D, Lanier LL, Parham P (1997). Human diversity in killer cell inhibitory receptor genes. Immunity. 7(6):753-63

Uhrberg M, Valiante NM, Young NT, Lanier LL, Phillips JH, Parham P (2001). The repertoire of killer cell Ig-like receptor and CD94:NKG2A receptors in T cells: clones sharing identical alpha beta TCR rearrangement express highly diverse killer cell Ig-like receptor patterns. J Immunol. 166(6):3923-32

UNAIDS (2008). Report on the global AIDS epidemic.

(http://www.unaids.org/en/KnowledgeCentre/HIVData/GlobalReport/2008/2008_Global_rep ort.asp) 
Urvater JA, Otting N, Loehrke JH, Rudersdorf R, Slukvin II, Piekarczyk MS, Golos TG, Hughes AL, Bontrop RE, Watkins DI (2000). Mamu-I: a novel primate MHC class I Brelated locus with unusually low variability. J Immunol. 164(3):1386-98

Valiante NM, Uhrberg M, Shilling HG, Lienert-Weidenbach K, Arnett KL, D'Andrea A, Phillips JH, Lanier LL, Parham P (1997). Functionally and structurally distinct NK cell receptor repertoires in the peripheral blood of two human donors. Immunity. 7(6):739-51

Vély F, Vivier E (1997). Conservation of structural features reveals the existence of a large family of inhibitory cell surface receptors and noninhibitory/activatory counterparts. $J$ Immunol. 159(5):2075-7

Vilches C, Pando MJ, Rajalingam R, Gardiner CM, Parham P (2000). Discovery of two novel variants of KIR2DS5 reveals this gene to be a common component of human KIR 'B' haplotypes. Tissue Antigens. 56(5):453-6

Vilches C, Parham P (2002). KIR: diverse, rapidly evolving receptors of innate and adaptive immunity. Annu Rev Immunol. 20:217-51

Vivier E, Nunès JA, Vély F (2004). Natural killer cell signaling pathways. Science. 306(5701):1517-9

Vivier E, Raulet DH, Moretta A, Caligiuri MA, Zitvogel L, Lanier LL, Yokoyama WM, Ugolini S (2011). Innate or adaptive immunity? The example of natural killer cells. Science. 331(6013):44-9

Wagtmann N, Biassoni R, Cantoni C, Verdiani S, Malnati MS, Vitale M, Bottino C, Moretta L, Moretta A, Long EO (1995). Molecular clones of the p58 NK cell receptor reveal immunoglobulin-related molecules with diversity in both the extra- and intracellular domains. Immunity. 2: 439-459

Walker BD (2007). Elite control of HIV Infection: implications for vaccines and treatment. Top HIV Med. 15(4):134-6

Welch AY, Kasahara M, Spain LM (2003). Identification of the mouse killer immunoglobulin-like receptor-like (Kirl) gene family mapping to chromosome $\mathrm{X}$. Immunogenetics. 54(11):782-90

Wende H, Colonna M, Ziegler A, Volz A (1999). Organization of the leukocyte receptor cluster (LRC) on human chromosome 19q13.4. Mamm Genome. 10(2):154-60

Wende H, Volz A, Ziegler A (2000). Extensive gene duplications and a large inversion characterize the human leukocyte receptor cluster. Immunogenetics. 51(8-9):703-13

Westgaard IH, Berg SF, Orstavik S, Fossum S, Dissen E (1998). Identification of a human member of the Ly-49 multigene family. Eur J Immunol. 28(6):1839-46

Wilson MJ, Torkar M, Haude A, Milne S, Jones T, Sheer D, Beck S, Trowsdale J (2000). Plasticity in the organization and sequences of human KIR/ILT gene families. Proc Natl Acad Sci USA. 97(9):4778-83 
Wilson MJ, Torkar M, Trowsdale J (1997). Genomic organization of a human killer cell inhibitory receptor gene. Tissue Antigens. 49(6):574-9

Wiseman RW, Karl JA, Bimber BN, O'Leary CE, Lank SM, Tuscher JJ, Detmer AM, Bouffard P, Levenkova N, Turcotte CL, Szekeres E Jr, Wright C, Harkins T, O'Connor DH (2009). Major histocompatibility complex genotyping with massively parallel pyrosequencing. Nat Med. 15(11):1322-1326

Wolthers KC, Otto SA, Lens SM, Van Lier RA, Miedema F, Meyaard L (1997). Functional B cell abnormalities in HIV type 1 infection: role of CD40L and CD70. AIDS Res Hum Retroviruses. 13(12):1023-9

Yant LJ, Friedrich TC, Johnson RC, May GE, Maness NJ, Enz AM, Lifson JD, O'Connor DH, Carrington M, Watkins DI (2006). The high-frequency major histocompatibility complex class I allele Mamu-B*17 is associated with control of simian immunodeficiency virus SIVmac239 replication. J Virol. 80(10):5074-7

Yawata M, Yawata N, Draghi M, Little AM, Partheniou F, Parham P (2006). Roles for HLA and KIR polymorphisms in natural killer cell repertoire selection and modulation of effector function. J Exp Med. 203(3):633-45

Yokoyama WM (2002). The search for the missing 'missing-self' receptor on natural killer cells. Scand J Immunol. 55(3):233-7

Yokoyama WM, Kim S, French AR (2004). The dynamic life of natural killer cells. Annu Rev Immunol. 22:405-29

Zhang ZQ, Fu TM, Casimiro DR, Davies ME, Liang X, Schleif WA, Handt L, Tussey L, Chen M, Tang A, Wilson KA, Trigona WL, Freed DC, Tan CY, Horton M, Emini EA, Shiver JW (2002). Mamu-A*01 allele-mediated attenuation of disease progression in simian-human immunodeficiency virus infection. J Virol. 76(24): 2845-12854 


\section{Supplement}

\subsection{KIR transcription profiles}

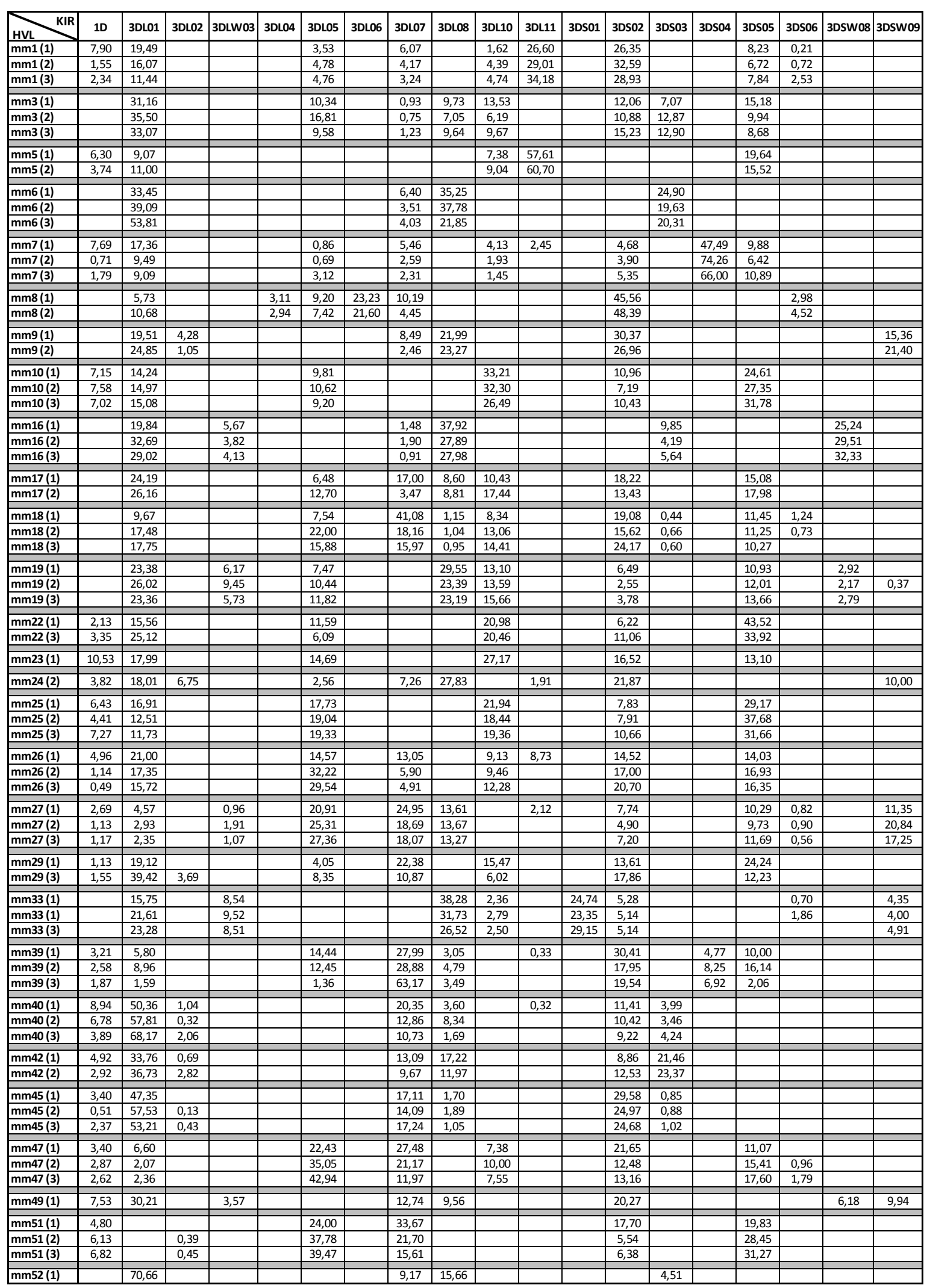




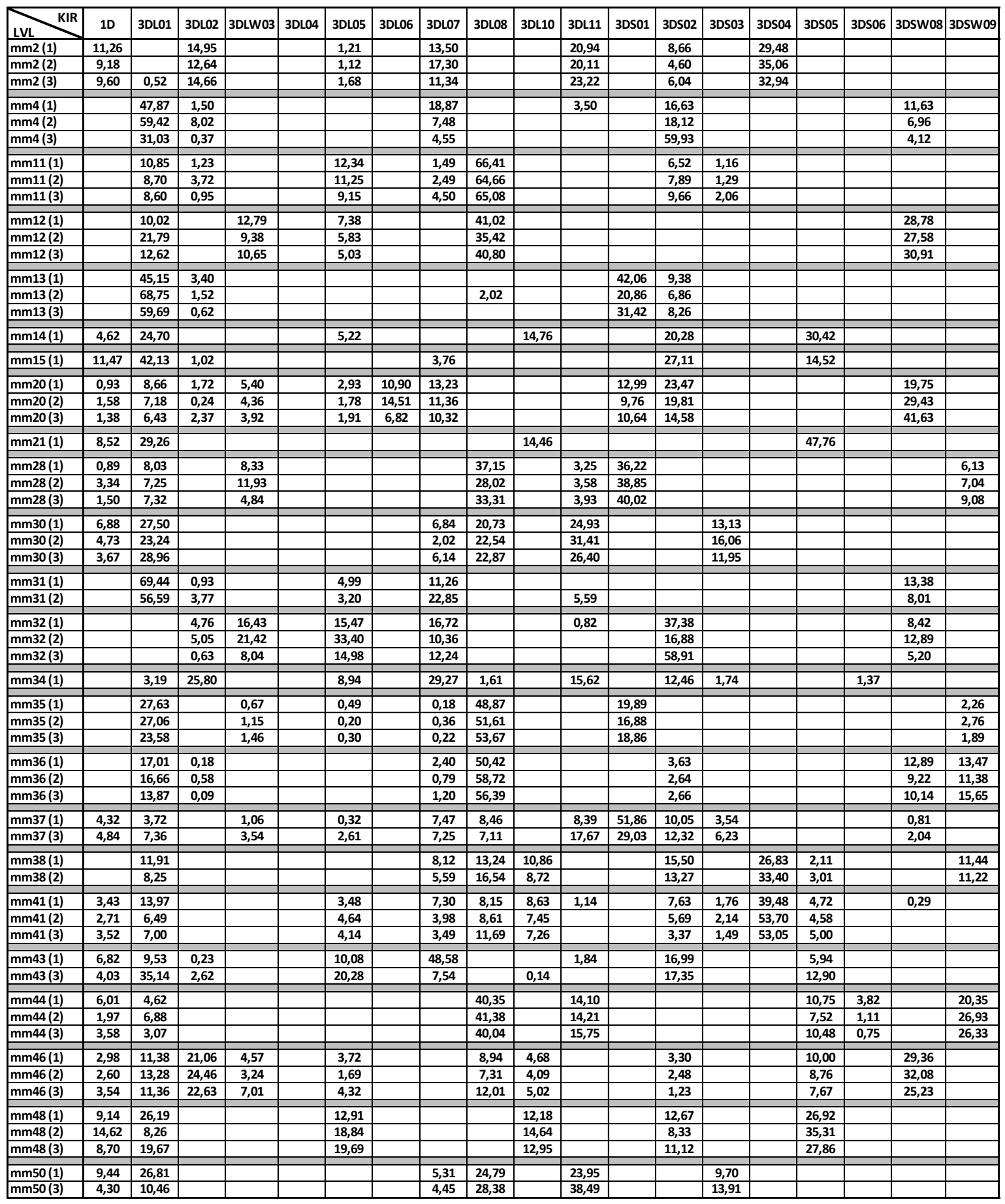

The KIR transcription profiles established by 454 sequencing of cDNA-PCR amplicons of the rhesus macaques are shown for both cohorts (HVL, LVL). The 19 distinct KIR transcripts detected by are listed on the top. On the left panel the animals are listed (mm1-52) along with the sample number $(1=$ pre infection; $2=$ acute infection; $3=$ chronic infection $)$. Abundance of $K I R$ sequences is given as a percentage of the total reads analysed per animal/sample, prior to removal of sequences comprising $\leq 0.9 \%$ of total reads. 


\subsection{MHC class I transcription profiles}

The $M H C$ class I transcription profiles established by 454 sequencing of 367 bp cDNA-PCR amplicons of the rhesus macaque cohort are shown for the HVL and LVL cohort. Only pre infection samples were used (mm24 acute infection sample). The MHC class I alleles detected are listed on the left panel and the animals are listed (mm1-52) on the top. Abundance of MHC class I sequences is given as a percentage of the total reads analysed per animal, prior to the removal of sequences comprising $\leq 1 \%$ of total sequencing reads. The different transcription levels are highlighted by colours (see below each table). Where multiple sequences are noted, the $M H C$ class I allele is ambiguous due to sequence identity within the 367 bp region examined. 


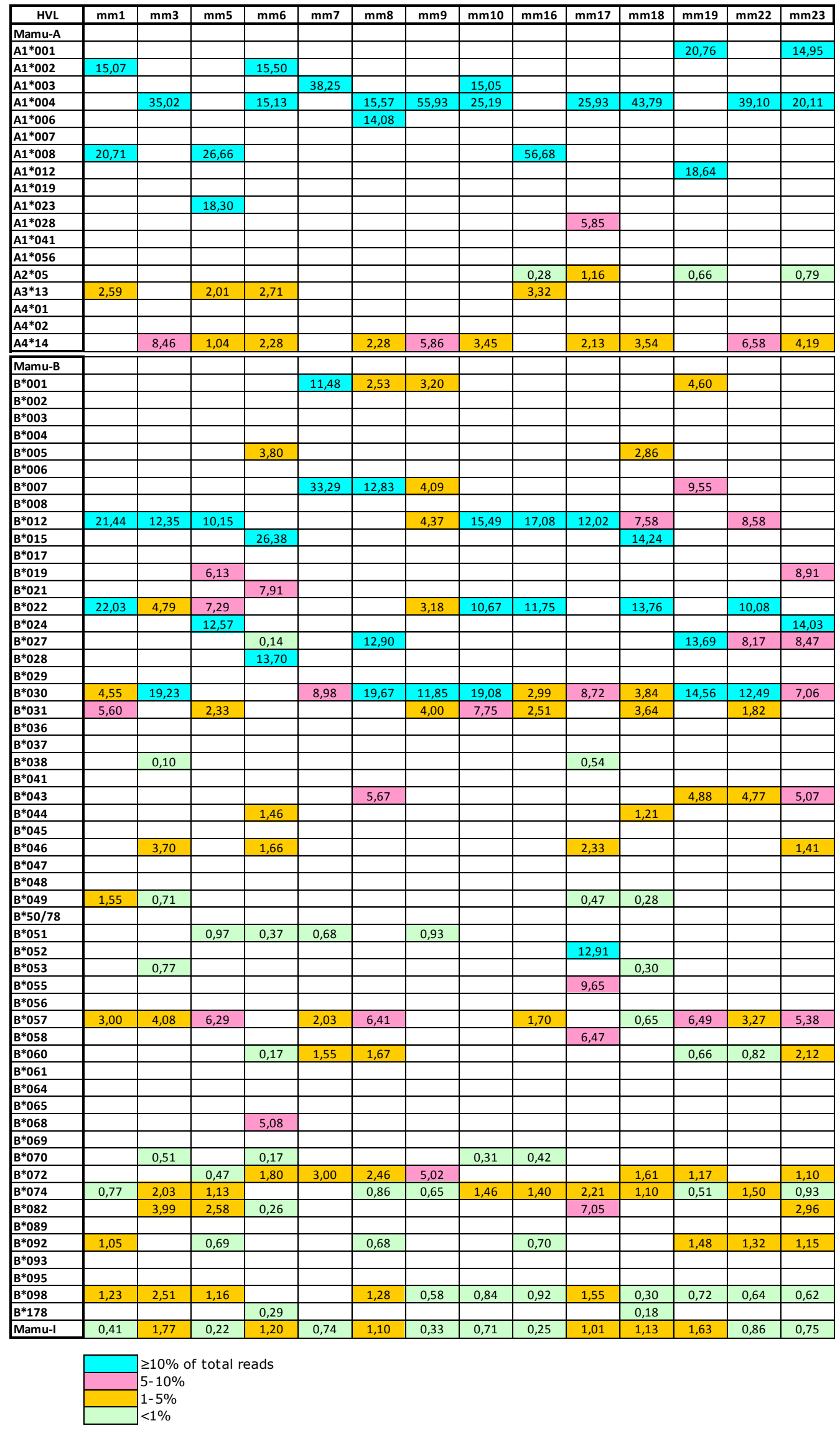




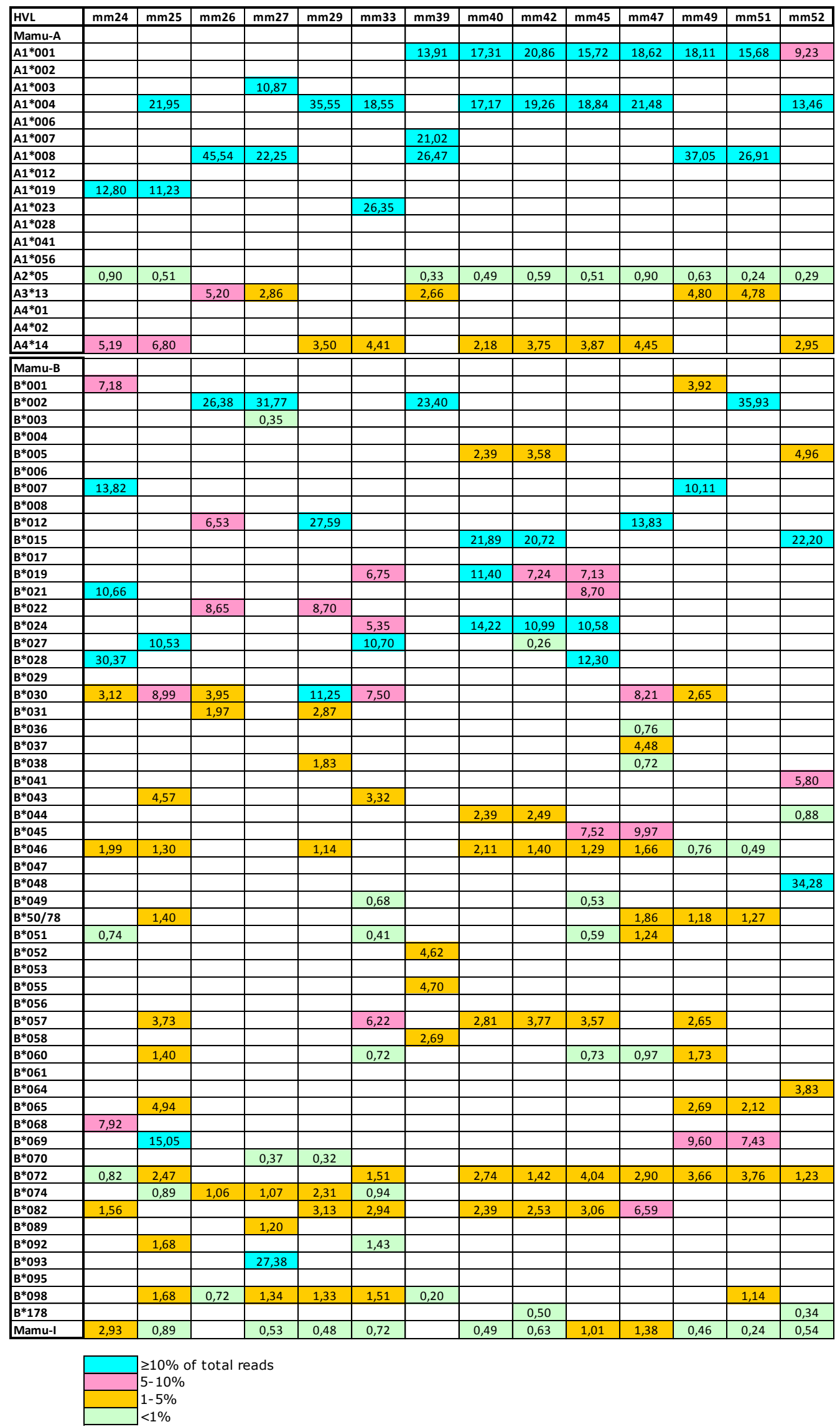




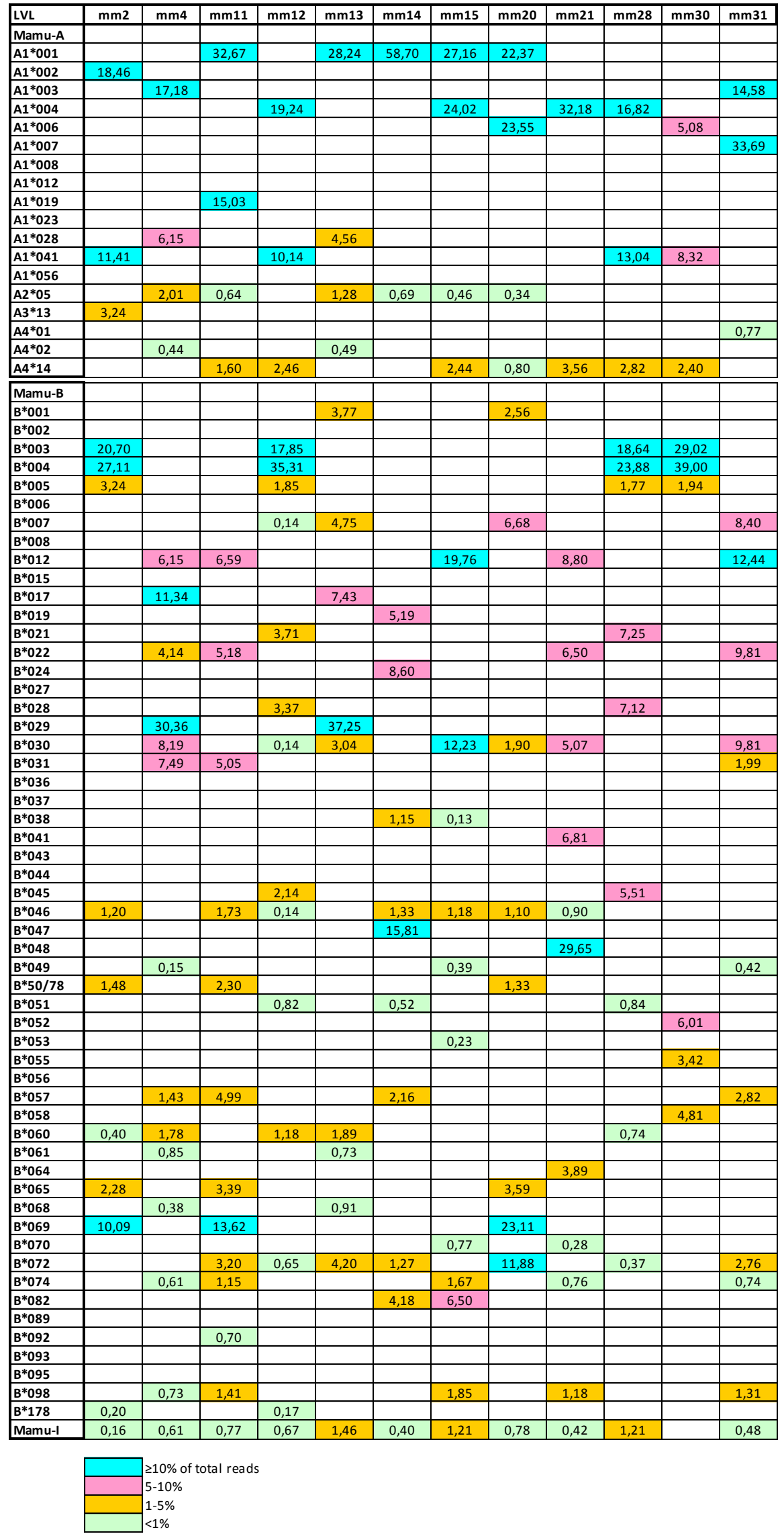




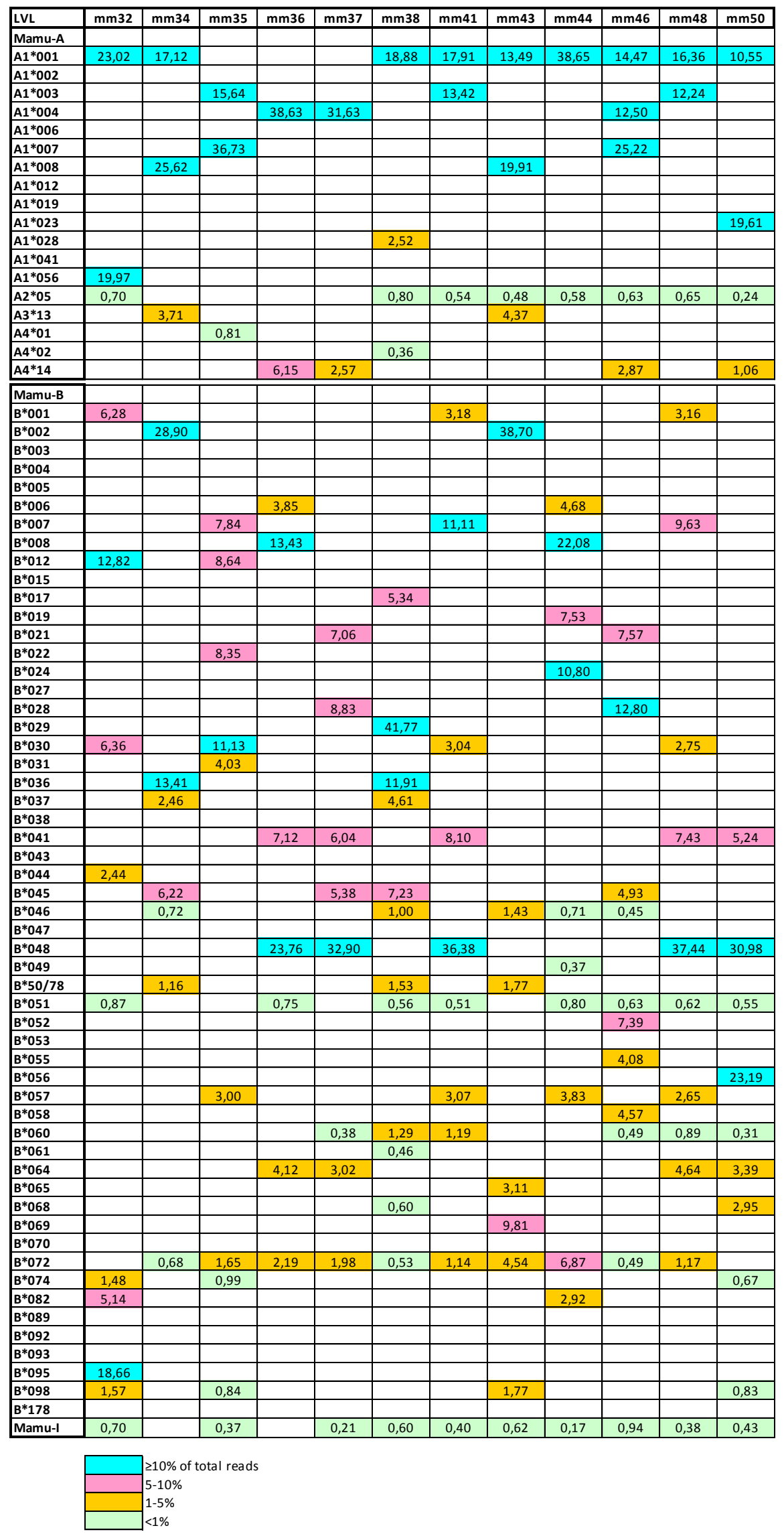




\subsection{Gene expression analyses (fold changes post infection with SIV)}

Relative gene expression values, which were determined using the $2^{-\Delta \Delta \mathrm{CT}}$ method of Livak and Schmittgen (2001) are shown. GAPDH was used as the reference gene to determine the delta $\mathrm{C}_{\mathrm{T}}\left(\Delta \mathrm{C}_{\mathrm{T}}\right)$ values, which were obtained directly from the data collection software (Fluidigm). Pre infection samples were used as the reference sample for each gene and individual to calculate $\Delta \Delta \mathrm{C}_{\mathrm{T}}$ values. The genes are listed on the top and the animals/samples $(2=$ acute infection sample; $3=$ chronic infection sample $)$ are listed on the left panel. Few genes failed to be amplified by the used approach and are not shown. Moreover, non-specific amplified genes were excluded, which was checked by melt curve analyses. Positive values indicate the fold changed post infection compared to the pre infection sample. Negative values indicate reduced mRNA expression compared to the pre infection sample. 


\begin{tabular}{|c|c|c|c|c|c|c|c|c|c|c|c|c|c|c|c|c|c|c|c|c|}
\hline HVL & $\begin{array}{ll}\text { CCR7 } \\
\end{array}$ & $\begin{array}{l}\text { CD14 } \\
\end{array}$ & \begin{tabular}{ll|} 
CD160 \\
\end{tabular} & CD19 & CD223 & CD244 & $\begin{array}{l}\text { CD226 } \\
\end{array}$ & CD27 & CD28 & $\begin{array}{l}\text { CD3D } \\
\end{array}$ & CD3G & $\overline{\text { CD30L }}$ & $\begin{array}{l}\text { CD38 } \\
\end{array}$ & CD4 & CD40 & $\overline{\text { CD40L }}$ & $\begin{array}{l}\text { CD69 } \\
\end{array}$ & \begin{tabular}{l|} 
CD70 \\
\end{tabular} & \begin{tabular}{|l|} 
CD80 \\
\end{tabular} & CD86 \\
\hline mm1 (2) & 3,81 & $-1,85$ & 8,00 & $-1,67$ & 13,83 & 18,13 & 3,63 & 3,18 & 1,57 & 1,93 & 4,96 & 6,19 & 11,24 & 10,85 & $-1,27$ & 10,56 & $-2,41$ & $-32,22$ & 1,14 & 2,85 \\
\hline mm1 (3) & 2,13 & 1,16 & 4,11 & $-2,07$ & 17,88 & 11,39 & 1,84 & 2,64 & 1,91 & 2,14 & 4,03 & 5,74 & 5,31 & 9,58 & 1,06 & 3,41 & $-3,16$ & $-19,16$ & $-2,68$ & 2,55 \\
\hline mm3 (2) & $-1,74$ & 1,28 & 2,68 & $-2,11$ & 14,22 & 10,56 & 2,23 & 4,20 & $-1,53$ & 5,13 & 7,16 & $-1,31$ & 8,51 & 1,99 & $-3,58$ & $-1,58$ & 2,33 & 1,01 & $-4,69$ & 3,01 \\
\hline mm3 (3) & $-2,07$ & 1,25 & 3,14 & $-8,34$ & 54,19 & 5,54 & 1,54 & 2,22 & $-6,19$ & 9,85 & 6,06 & $-1,08$ & 5,70 & $-2,11$ & $-3,25$ & 1,21 & $-2,19$ & 1,52 & $-6,19$ & 2,16 \\
\hline mm5 (2) & $-3,16$ & 1,02 & 6,73 & $-1,37$ & 6,73 & 9,13 & 2,11 & 3,92 & $-1,35$ & 2,22 & 3,27 & $-1,57$ & 3,73 & 3,27 & $-2,36$ & $-2,89$ & $-2,17$ & $-1,56$ & $-9,45$ & $-1,45$ \\
\hline mm6 (2) & \begin{tabular}{|l|}
$-2,08$ \\
\end{tabular} & 1,27 & 15,45 & 1,13 & 7,62 & 13,00 & 3,43 & 3,66 & 1,59 & 3,20 & 5,74 & 1,53 & 5,31 & 6,87 & 1,39 & 1,83 & 2,11 & $-92,41$ & $-4,99$ & 4,35 \\
\hline mm6 (3) & $-2,16$ & $-1,61$ & 6,73 & 2,87 & 10,13 & 8,51 & 2,22 & 3,61 & 1,16 & 4,20 & 4,79 & 1,02 & 5,31 & 2,66 & 3,03 & 5,06 & 1,24 & $-46,21$ & $-2,99$ & 3,81 \\
\hline mm7 (2) & $-2,62$ & $-1,08$ & 8,94 & 1,29 & 7,06 & 8,22 & $-1,06$ & 1,51 & $-3,78$ & 1,21 & 2,22 & $-1,51$ & 7,52 & 1,33 & $-1,32$ & $-1,09$ & $-4,41$ & \begin{tabular}{|c|}
$-2,20$ \\
\end{tabular} & $-5,10$ & 1,66 \\
\hline mm7 (3) & $-27,28$ & 1,44 & 1,23 & 1,65 & $-1,12$ & 2,38 & $-5,35$ & 2,14 & $-56,89$ & $-3,27$ & $-2,16$ & $-5,39$ & 1,62 & $-1,19$ & $-2,35$ & $-7,57$ & $-17,75$ & $-1,95$ & $-5,86$ & 2,03 \\
\hline mm8 (2) & $-6,68$ & $-1,18$ & 9,58 & $-1,44$ & 9,99 & 8,40 & $-1,14$ & 2,17 & $-1,33$ & 1,15 & 2,97 & $-1,62$ & 2,50 & 2,97 & $-3,61$ & $-1,51$ & 1,44 & $-116,16$ & $-3,92$ & 1,49 \\
\hline mm9 (2) & $-2,20$ & 2,00 & 13,93 & $-2,22$ & 11,39 & 12,04 & 2,50 & 5,54 & $-1,91$ & 3,07 & 5,06 & 2,13 & 14,62 & 2,66 & $-1,28$ & 3,46 & $-1,17$ & $-28,25$ & $-2,45$ & 2,57 \\
\hline mm10 (2) & $-2,06$ & 1,40 & 3,94 & $-1,60$ & 4,96 & 9,45 & 2,13 & 2,10 & $-1,56$ & 2,11 & 4,11 & $-1,21$ & 4,03 & 2,69 & $-2,62$ & $-1,43$ & $-3,01$ & $-1,51$ & $-5,86$ & 1,46 \\
\hline $\operatorname{mm10}(3)$ & 1,52 & $-1,18$ & 5,74 & $-1,92$ & 13,93 & 9,99 & 2,35 & 3,10 & $-1,06$ & 3,16 & 5,98 & 1,65 & 7,11 & 4,20 & $-1,02$ & 2,11 & $-1,49$ & $-12,47$ & $-5,10$ & 3,10 \\
\hline mm17 (2) & $-9,25$ & 2,93 & 9,25 & $-6,02$ & 5,86 & 3,36 & $-1,71$ & 1,44 & $-2,83$ & 3,18 & 1,21 & $-1,22$ & 2,36 & $-1,37$ & $-2,95$ & $-3,89$ & $-1,36$ & 1,88 & $-9,65$ & $-1,14$ \\
\hline mm18 (2) & \begin{tabular}{|l|}
$-2,39$ \\
\end{tabular} & 1,62 & 8,34 & $-2,66$ & 17,51 & 4,89 & $-1,05$ & 5,13 & $-2,50$ & 6,28 & 2,75 & 1,99 & 6,28 & $-1,07$ & $-4,06$ & 1,21 & $-1,48$ & $-2,23$ & $-4,86$ & 1,04 \\
\hline mm18 (3) & 1,01 & 1,53 & 12,82 & $-1,04$ & 16,11 & 4,82 & 1,48 & 4,96 & $-1,24$ & 3,23 & 2,17 & 3,53 & 7,36 & 2,06 & 1,05 & 4,53 & $-2,97$ & $-4,17$ & $-2,11$ & 1,95 \\
\hline mm19 (2) & \begin{tabular}{|c|}
$-7,26$ \\
\end{tabular} & 5,03 & 5,17 & $-2,57$ & 4,53 & 11,16 & $-1,60$ & 6,82 & \begin{tabular}{|l|}
$-100,43$ \\
\end{tabular} & 7,36 & 3,05 & 1,12 & 5,21 & $-1,71$ & 2,07 & 7,06 & $-1,61$ & 2,04 & $-3,25$ & 1,57 \\
\hline $\operatorname{mm19}(3)$ & $-5,31$ & 5,24 & $-1,66$ & $-1,53$ & 1,40 & 1,74 & $-2,55$ & 2,77 & $-81,57$ & 2,51 & 1,80 & $-1,01$ & 3,51 & 0,00 & 1,24 & 1,03 & $-2,30$ & 4,50 & $-4,17$ & $-1,08$ \\
\hline mm22 (3) & $\begin{array}{l}-6,92 \\
\end{array}$ & $-1,88$ & $-2,85$ & 3,51 & 0,00 & $-3,01$ & $-7,31$ & 1,93 & $-4,26$ & $-4,00$ & $-2,60$ & $-4,56$ & 2,22 & $-5,28$ & 1,64 & $-11,79$ & $-1,77$ & 3,97 & 2,30 & 3,12 \\
\hline mm25 (2) & \begin{tabular}{|c|}
$-3,73$ \\
\end{tabular} & \begin{tabular}{|l|}
$-1,52$ \\
\end{tabular} & 5,24 & $-2,35$ & 4,06 & 6,63 & 3,46 & 2,66 & $-2,57$ & 1,27 & 4,63 & $-1,26$ & 3,27 & 3,48 & $-4,20$ & $-1,25$ & $-1,06$ & $-1,87$ & $-2,11$ & 1,27 \\
\hline $\operatorname{mm} 25(3)$ & $-30,70$ & 1,21 & 3,41 & $-13,93$ & 1,89 & 2,53 & $-1,11$ & $-1,61$ & $-21,41$ & $-1,31$ & $-1,25$ & $-6,87$ & 1,48 & $-4,96$ & $-16,56$ & $-9,65$ & $-7,36$ & 3,56 & $-7,36$ & $-1,99$ \\
\hline $\operatorname{mm26}(2)$ & \begin{tabular}{|l|}
$-2,19$ \\
\end{tabular} & 2,60 & 5,98 & $-1,22$ & 6,36 & nd & 4,79 & 3,01 & 1,15 & $\begin{array}{ll}1,59 \\
\end{array}$ & 4,72 & 1,78 & 3,36 & 5,24 & \begin{tabular}{|l|}
$-3,81$ \\
\end{tabular} & $-1,08$ & 1,20 & $-4,32$ & $\begin{array}{l}-4,32 \\
\end{array}$ & 2,79 \\
\hline $\operatorname{mm26}(3)$ & $-1,60$ & 2,45 & 11,31 & $-4,17$ & 16,00 & nd & 3,05 & 1,82 & $-2,41$ & 2,20 & 3,46 & 1,38 & 3,61 & 2,48 & $-3,48$ & 1,61 & $-1,26$ & $-8,11$ & $-7,11$ & 2,46 \\
\hline mm27 (2) & \begin{tabular}{|l|}
$-15,03$ \\
\end{tabular} & 1,75 & 1,80 & $-1,97$ & 2,57 & 7,01 & $-1,13$ & $-1,52$ & \begin{tabular}{|l|}
$-13,45$ \\
\end{tabular} & $\begin{array}{ll}-1,52 \\
\end{array}$ & 1,35 & $-1,37$ & 1,08 & 1,83 & $\begin{array}{l}-5,86 \\
\end{array}$ & $-3,39$ & $-2,08$ & $-1,54$ & $\begin{array}{l}-3,66 \\
\end{array}$ & 1,51 \\
\hline $\operatorname{mm} 27(3)$ & $-6,68$ & 2,17 & 2,58 & $-2,27$ & 7,16 & 20,39 & 1,18 & $-1,13$ & $-17,03$ & 1,01 & 2,13 & $-2,17$ & 1,77 & 1,11 & $-2,46$ & $-1,99$ & $-4,17$ & $-7,01$ & $-8,00$ & 1,18 \\
\hline mm29 (3) & \begin{tabular}{|c|}
$-5,66$ \\
\end{tabular} & \begin{tabular}{|l|}
$-3,58$ \\
\end{tabular} & $-1,40$ & 2,46 & 2,01 & $-1,18$ & $-2,43$ & 1,48 & \begin{tabular}{|c|}
$-3,36$ \\
\end{tabular} & \begin{tabular}{|l|}
$-3,34$ \\
\end{tabular} & $-1,51$ & $-2,17$ & 1,29 & $-2,62$ & $-1,25$ & \begin{tabular}{|l|}
$-17,63$ \\
\end{tabular} & $-1,92$ & $-10,06$ & 1,88 & 2,53 \\
\hline mm33 (2) & $-1,77$ & 2,89 & 8,28 & $-1,82$ & 8,17 & 7,73 & 1,49 & 3,89 & $-2,91$ & \begin{tabular}{|l|l|}
5,35 \\
\end{tabular} & 6,06 & 1,30 & 5,98 & 1,72 & 1,04 & 8,57 & 1,46 & \begin{tabular}{|l|}
$-2,07$ \\
\end{tabular} & $-6,28$ & 1,54 \\
\hline mm33 (3) & $-1,58$ & $-1,05$ & 6,87 & $-1,16$ & 12,13 & 5,28 & 2,03 & 5,06 & $-3,25$ & 6,11 & 6,87 & $-1,06$ & 6,28 & 2,91 & 1,37 & 3,27 & $-1,06$ & $-4,32$ & $-6,50$ & $-1,34$ \\
\hline mm39 (2) & $-1,22$ & 2,46 & 5,24 & 1,42 & 1,9 & 5,54 & 1,19 & 1,30 & 0,00 & 1,54 & 3,03 & $-1,04$ & 3,84 & 2,62 & 1,54 & 1,47 & 1,27 & $-83,29$ & $-8,94$ & 2,23 \\
\hline mm39 (3) & $-28,05$ & 2,73 & 2,14 & 1,73 & 1,61 & 1,34 & $-5,06$ & 1,33 & $-17,63$ & $-2,45$ & $-2,00$ & $-3,20$ & $-1,43$ & $-2,07$ & $-1,88$ & $-15,89$ & $-6,59$ & $-20,68$ & $-5,58$ & 2,36 \\
\hline mm40 (2) & \begin{tabular}{|l|}
$-1,78$ \\
\end{tabular} & 1,22 & 2,75 & 1,79 & 3,18 & 7,36 & 3,32 & 2,20 & \begin{tabular}{|l|}
$-1,39$ \\
\end{tabular} & 1,08 & 3,14 & 1,88 & 5,66 & 6,96 & $-1,44$ & 2,23 & $-1,56$ & $-116,16$ & $-2,22$ & 3,23 \\
\hline mm40 (3) & $-3,58$ & 1,56 & 2,79 & 10,85 & 3,81 & 4,82 & 1,21 & 4,44 & $-2,91$ & $-1,03$ & 2,20 & 1,46 & 3,61 & 3,05 & 1,95 & $-2,95$ & $-1,53$ & $-26,17$ & 1,20 & 2,99 \\
\hline $\mathrm{mm45}(2)$ & \begin{tabular}{|l|}
$-1,79$ \\
\end{tabular} & 1,67 & 4,20 & 2,57 & 2,36 & 7,94 & 2,11 & 1,95 & \begin{tabular}{|l|}
$-1,85$ \\
\end{tabular} & \begin{tabular}{|l|}
$-1,25$ \\
\end{tabular} & 2,39 & $-1,34$ & 4,59 & 6,41 & 1,38 & 1,42 & \begin{tabular}{|l|}
$-3,03$ \\
\end{tabular} & $-167,73$ & $\begin{array}{l}-6,06 \\
\end{array}$ & $\begin{array}{l}-1,41 \\
\end{array}$ \\
\hline mm45 (3) & $-1,80$ & 0,00 & 2,91 & 15,67 & 3,03 & 9,32 & 2,45 & 6,23 & $-1,52$ & $-1,19$ & 3,71 & 1,82 & 7,62 & 7,26 & 3,14 & $-1,46$ & $-1,78$ & $-24,59$ & 1,45 & 1,60 \\
\hline $\mathrm{mm} 47$ (2) & \begin{tabular}{|l|}
$-1,92$ \\
\end{tabular} & $-1,15$ & 4,14 & 4,20 & 4,35 & 8,34 & 2,51 & 5,43 & 1,23 & 1,23 & 9,25 & $-2,23$ & 4,08 & 8,00 & 2,87 & 2,04 & $-2,39$ & $-45,57$ & $-4,76$ & 2,41 \\
\hline mm47 (3) & $-2,25$ & 1,17 & 1,32 & 2,00 & 4,00 & 6,63 & 1,33 & 3,20 & $-1,32$ & $-1,73$ & 3,25 & $-2,53$ & 2,10 & 4,38 & 1,39 & $-2,53$ & $-1,48$ & $-44,32$ & $-7,41$ & 2,83 \\
\hline mm49 (2) & \begin{tabular}{|l|}
$-3,39$ \\
\end{tabular} & 1,04 & $-1,06$ & 2,68 & $-1,96$ & 1,88 & 1,14 & 1,84 & 1,80 & 1,01 & 1,96 & $-1,30$ & 1,22 & 1,91 & 1,01 & $-1,62$ & $-1,20$ & 1,09 & $-2,48$ & 1,05 \\
\hline mm49 (3) & $-1,20$ & $-1,68$ & 1,66 & 8,94 & 2,85 & 4,63 & 2,35 & 6,28 & 1,22 & $-1,24$ & 3,20 & $-1,46$ & 4,23 & 4,14 & 2,97 & $-1,15$ & $-1,68$ & $-4,79$ & 1,64 & 2,04 \\
\hline mm52 (2) & 2,10 & $-2,41$ & 2,62 & 1,82 & 3,58 & 2,77 & 2,06 & 2,28 & 1,54 & 1,14 & 3,03 & $-1,04$ & 3,56 & 5,10 & \begin{tabular}{|l|}
$-1,58$ \\
\end{tabular} & $-1,04$ & $-1,91$ & \begin{tabular}{|c|}
$-4,56$ \\
\end{tabular} & $-2,14$ & 1,15 \\
\hline mm52 (3) & 1,30 & $-2,97$ & 1,39 & 3,56 & 1,40 & 1,95 & 1,08 & 1,74 & $-1,75$ & $-1,53$ & 1,61 & $-1,03$ & 1,71 & 2,08 & 1,13 & $-4,38$ & $-4,69$ & $-2,68$ & 1,13 & 1,18 \\
\hline LVL & CCR7 & CD14 & CD160 & CD19 & CD223 & CD244 & CD226 & CD27 & $\begin{array}{ll}\text { CD28 } \\
\end{array}$ & CD3D & CD3G & CD30L & CD38 & CD4 & CD40 & CD40L & D69-lik & CD70 & CD80 & CD86 \\
\hline mm2 (2) & 1,85 & $-2,33$ & 2,31 & 1,31 & 4,92 & 4,99 & 2,23 & 1,47 & 3,84 & 1,04 & 2,22 & 1,66 & 4,38 & 7,31 & $-1,26$ & 1,88 & 1,16 & $-15,24$ & 0,00 & 1,80 \\
\hline mm2 (3) & $-1,11$ & 1,83 & 2,30 & 1,67 & 3,18 & 3,63 & 1,48 & 1,42 & 2,00 & 1,58 & 1,96 & 1,59 & 2,97 & 6,54 & 1,67 & 2,43 & $-1,35$ & 1,21 & $-2,27$ & 1,08 \\
\hline mm4 (2) & $-3,14$ & 2,07 & 12,04 & $-3,43$ & 8,06 & 3,23 & $1,4 \mathrm{c}$ & 4,50 & $-1,32$ & 4,11 & 3,86 & $-1,21$ & 2,99 & 2,00 & $-2,50$ & $-1,32$ & 1,23 & \begin{tabular}{|c|}
$-61,39$ \\
\end{tabular} & $-3,29$ & 2,06 \\
\hline mm4 (3) & $-1,49$ & 1,33 & 12,55 & 4,53 & 8,75 & 5,31 & 1,48 & 7,62 & $-1,34$ & 2,55 & 5,70 & 1,28 & 2,28 & 4,63 & 1,39 & 1,65 & $-1,36$ & $-36,00$ & $-1,17$ & 4,79 \\
\hline mm11 (2) & 1,65 & \begin{tabular}{|l|}
$-1,36$ \\
\end{tabular} & $-1,13$ & 2,19 & 1,54 & $-1,49$ & 1,72 & $-1,30$ & 1,27 & \begin{tabular}{|l|}
$-1,23$ \\
\end{tabular} & $-1,39$ & 1,19 & 1,88 & 3,43 & 1,83 & \begin{tabular}{ll|}
$-1,21$ \\
\end{tabular} & 1,20 & 1,34 & 2,03 & $\begin{array}{l}-1,37 \\
\end{array}$ \\
\hline mm11 (3) & 2,17 & $-2,79$ & $-1,92$ & 1,57 & 1,47 & $-2,03$ & 1,49 & $-1,09$ & 1,15 & $-1,29$ & $-1,17$ & 1,02 & 1,34 & 4,82 & 1,47 & 1,05 & 3,10 & $-1,26$ & 2,22 & $-1,69$ \\
\hline $\mathrm{mm12}(2)$ & $-4,99$ & \begin{tabular}{|l|}
$-3,46$ \\
\end{tabular} & \begin{tabular}{|l|}
13,09 \\
\end{tabular} & \begin{tabular}{|l|}
$-1,54$ \\
\end{tabular} & 4,08 & 3,29 & 1,06 & 1,57 & \begin{tabular}{|l|}
$-2,39$ \\
\end{tabular} & \begin{tabular}{|l|}
$-1,80$ \\
\end{tabular} & 1,54 & $\begin{array}{ll}1,04 \\
\end{array}$ & 4,17 & 1,74 & \begin{tabular}{|l|}
$-3,27$ \\
\end{tabular} & \begin{tabular}{l|l|}
$-1,48$ \\
\end{tabular} & \begin{tabular}{|l|}
$-1,38$ \\
\end{tabular} & \begin{tabular}{|l|}
$-5,98$ \\
\end{tabular} & $-4,06$ & 1,85 \\
\hline $\operatorname{mm12}(3)$ & $-2,51$ & $-1,21$ & 12,21 & 3,20 & 6,73 & 6,63 & $-1,02$ & 5,35 & $-2,31$ & 1,88 & 3,89 & 1,07 & 4,56 & 3,78 & 3,39 & 2,81 & 1,08 & $-41,64$ & $-3,41$ & 2,22 \\
\hline mm20 (2) & \begin{tabular}{|l|}
$-2,38$ \\
\end{tabular} & \begin{tabular}{|l|}
$-1,45$ \\
\end{tabular} & $-3,53$ & $-1,68$ & $-4,66$ & $\begin{array}{l}-6,68 \\
\end{array}$ & $-1,68$ & $\begin{array}{l}-2,51 \\
\end{array}$ & $-1,62$ & \begin{tabular}{|l|}
$-3,66$ \\
\end{tabular} & $-3,12$ & $-1,18$ & $-1,52$ & 1,34 & \begin{tabular}{|c|}
$-3,89$ \\
\end{tabular} & $-5,82$ & $-1,25$ & $-1,09$ & $\begin{array}{l}-4,00 \\
\end{array}$ & $\begin{array}{l}-1,39 \\
\end{array}$ \\
\hline $\operatorname{mm20}(3)$ & $-8,57$ & $-2,60$ & $-4,63$ & $-1,04$ & $-2,95$ & $-1,59$ & $-2,62$ & $-1,01$ & $-4,50$ & $-2,87$ & $-2,03$ & $-3,51$ & $-3,48$ & $-2,16$ & $-3,43$ & $-9,92$ & $-2,45$ & 7,21 & $-1,57$ & $-2,13$ \\
\hline $\mathrm{mm} 28$ (2) & \begin{tabular}{|l|}
$-1,34$ \\
\end{tabular} & \begin{tabular}{|l|}
$-1,88$ \\
\end{tabular} & 4,56 & 1,38 & 4,53 & 9,32 & 3,23 & 2,51 & \begin{tabular}{|l|}
$-1,17$ \\
\end{tabular} & 1,91 & 2,55 & 2,77 & 8,82 & 4,72 & 1,43 & $\begin{array}{ll}3,39 \\
\end{array}$ & $\begin{array}{l}2,33 \\
\end{array}$ & $-39,67$ & $-2,27$ & 2,83 \\
\hline $\operatorname{mm} 28(3)$ & $-2,93$ & 1,26 & 5,94 & 2,64 & 4,29 & 5,5 & 3,29 & 3,66 & $-1,71$ & 2,95 & 3,07 & $-1,15$ & 4,53 & 1,99 & 3,18 & 1,80 & $-2,07$ & $-93,70$ & $-2,41$ & 2,11 \\
\hline $\mathrm{mm30}(2)$ & \begin{tabular}{|c|}
$-3,71$ \\
\end{tabular} & 2,08 & 3,16 & 1,37 & 6,63 & 8,5 & $\overline{1,0}$ & 2,99 & $-1,08$ & 2,30 & 2,97 & $-1,33$ & 2,23 & 2,87 & $-1,36$ & $-1,04$ & $-1,93$ & $-5,21$ & $\begin{array}{l}-3,56 \\
\end{array}$ & $-1,13$ \\
\hline mm30 (3) & $-2,66$ & 3,61 & 5,58 & 1,75 & 4,63 & 14,72 & 1,3 & 4,11 & $-2,68$ & 2,85 & 3,66 & 1,08 & 1,85 & 3,66 & 1,39 & 4,26 & $-3,34$ & $-8,75$ & $-2,22$ & 1,30 \\
\hline mm32 (2) & 1,19 & 1,13 & 3,66 & 1,21 & 4,1 & 24,76 & 5,94 & 3,66 & \begin{tabular}{|c|}
$-3,29$ \\
\end{tabular} & 2,30 & 5,86 & $-1,16$ & 2,22 & 7,06 & 2,62 & \begin{tabular}{|l|l|}
5,74 \\
\end{tabular} & $-3,12$ & $-56,10$ & $-2,99$ & 1,12 \\
\hline mm32 (3) & 1,69 & 1,62 & 2,07 & 2,45 & 5,28 & 8,00 & 4,99 & 4,03 & 1,39 & 2,48 & 5,50 & 1,35 & 3,16 & 8,00 & 4,99 & 3,23 & $-2,28$ & $-53,45$ & $-3,94$ & 2,30 \\
\hline mm35 (2) & $-2,60$ & \begin{tabular}{|l|}
$-1,33$ \\
\end{tabular} & $-1,10$ & $-2,27$ & 1,49 & $-1,38$ & $-1,58$ & $-1,30$ & $-1,61$ & 1,16 & $-1,87$ & $-1,34$ & 1,46 & $-2,16$ & 1,60 & $-1,39$ & $-1,28$ & \begin{tabular}{|l|}
$-2,83$ \\
\end{tabular} & 1,09 & 1,43 \\
\hline mm35 (3) & $-1,57$ & $-2,35$ & 0,00 & 2,14 & 2,16 & 1,34 & $-1,12$ & 1,55 & 1,71 & 1,87 & 1,04 & 1,02 & 1,19 & 1,01 & 4,92 & $-1,14$ & 1,91 & $-35,51$ & 1,89 & 1,97 \\
\hline mm36 (2) & $-1,02$ & 1,04 & 9,92 & $-1,09$ & 8,69 & 11,31 & 8,28 & 3,94 & $-2,91$ & 2,53 & 4,69 & $-1,02$ & 4,92 & 4,20 & $-2,07$ & 1,74 & $-1,27$ & \begin{tabular}{|l|l|}
$-74,54$ \\
\end{tabular} & $-1,53$ & 1,57 \\
\hline $\operatorname{mm} 36(3)$ & 1,85 & 1,95 & 8,57 & 4,86 & 3,94 & 5,90 & 10,27 & 5,43 & 1,30 & 2,19 & 8,11 & 1,13 & 4,50 & 9,06 & 1,44 & 2,00 & $-1,16$ & $-42,52$ & $-1,14$ & 4,82 \\
\hline mm37 (3) & 2,73 & $-9,65$ & 4,03 & 2,43 & 1,35 & 1,88 & 3,27 & 3,86 & 2,89 & 1,13 & 2,53 & $-1,60$ & 3,78 & 2,25 & 3,76 & 1,47 & 1,42 & $-66,72$ & 1,93 & $-1,04$ \\
\hline n38 (2) & \begin{tabular}{|l|}
$-1,38$ \\
\end{tabular} & \begin{tabular}{|l|l|}
$-1,18$ \\
\end{tabular} & 2,71 & 1,41 & 4,17 & 6,1 & 3,92 & 3,12 & $\begin{array}{l}-2,69 \\
\end{array}$ & 1,99 & 3,56 & $-1,48$ & 2,62 & 2,45 & 1,80 & 2,99 & $-1,88$ & $-27,67$ & $-1,35$ & 1,80 \\
\hline mm38 (3) & $-1,39$ & 1,40 & 1,69 & 2,85 & 3,94 & 10,48 & 1,61 & 3,10 & $-4,17$ & 1,41 & 2,97 & 1,35 & 5,46 & 3,97 & 2,55 & 1,10 & $-2,41$ & $-2,55$ & $-7,16$ & 3,41 \\
\hline mm41 (2) & 2,35 & \begin{tabular}{|l|}
$-1,31$ \\
\end{tabular} & \begin{tabular}{|l|}
19,29 \\
\end{tabular} & 65,34 & 13,64 & 35,02 & 1,96 & 8,82 & 2,45 & 2,83 & 7,06 & 1,04 & 10,85 & 19,56 & 6,77 & 3,18 & 1,60 & $-64,00$ & 1,80 & 1,45 \\
\hline mm41 (3) & 1,59 & 1,29 & 7,41 & 50,56 & 4,32 & 45,89 & 6,11 & 8,82 & $-1,77$ & 2,58 & 6,50 & $-1,49$ & 3,61 & 19,29 & 3,01 & 2,33 & $-1,40$ & $-89,26$ & 1,55 & 1,55 \\
\hline mm44 (2) & 1,30 & $-2,08$ & 9,32 & 5,39 & 6,28 & 21,41 & 9,78 & 5,82 & 1,23 & 2,14 & 4,76 & 1,16 & 7,21 & 10,34 & 1,83 & 3,01 & 1,46 & $-70,52$ & $-3,63$ & 1,51 \\
\hline mm44 (3) & 1,77 & 1,99 & 3,86 & 6,96 & 3,12 & 15,35 & 6,45 & 5,13 & 1,11 & 1,65 & 2,89 & 1,77 & 3,34 & 9,19 & 1,97 & 2,83 & $-1,64$ & $-17,51$ & $-1,95$ & 1,95 \\
\hline $\mathrm{mm} 46$ (2) & 1,18 & $-7,16$ & 3,73 & 2,04 & 2,75 & 4,11 & 1,87 & 2,68 & 1,16 & 1,28 & 2,50 & $-1,85$ & $-1,06$ & 1,42 & 2,17 & 1,54 & $-1,78$ & \begin{tabular}{|l|}
$-5,43$ \\
\end{tabular} & $-1,16$ & $-1,04$ \\
\hline $\mathrm{mm} 46(3)$ & $-1,01$ & $-1,16$ & 1,33 & 1,07 & 1,83 & 2,58 & 1,27 & 1,61 & 1,49 & $-1,24$ & 2,81 & $-1,88$ & $-1,16$ & 3,27 & $-1,56$ & 1,65 & $-2,51$ & $-1,44$ & $-3,14$ & $-1,40$ \\
\hline mm48 (2) & \begin{tabular}{|l|}
$-1,92$ \\
\end{tabular} & $-2,10$ & 1,04 & 6,77 & 1,22 & 2,93 & 1,06 & 2,33 & $\begin{array}{l}-1,99 \\
\end{array}$ & \begin{tabular}{|l|}
$-1,68$ \\
\end{tabular} & 1,64 & $-6,28$ & 0,00 & 2,99 & 1,45 & $-3,39$ & $-3,66$ & \begin{tabular}{|c|}
$-1,20$ \\
\end{tabular} & $-2,11$ & 1,06 \\
\hline mm48 (3) & $-1,06$ & $-6,41$ & 1,28 & 6,82 & 1,07 & 1,72 & 1,29 & 2,25 & $-2,00$ & $-2,22$ & 1,16 & $-2,27$ & $-1,04$ & 2,35 & 1,79 & 1,01 & $-4,72$ & $-5,90$ & 1,09 & 1,95 \\
\hline mm50 (3) & 2,31 & $-1,17$ & 1,42 & 9,71 & 1,14 & 4,29 & 2,69 & 11,47 & 2,35 & 1,88 & 6,59 & 1,40 & 4,76 & 18,38 & 1,25 & $\begin{array}{l}6,59 \\
\end{array}$ & $-2,73$ & $-2,17$ & $-3,48$ & 1,05 \\
\hline
\end{tabular}




\begin{tabular}{|c|c|c|c|c|c|c|c|c|c|c|c|c|c|c|c|c|c|c|c|c|c|}
\hline HVL & CD8B & CD94 & $\begin{array}{l}\text { CD96 } \\
\end{array}$ & FASLG & FCER1G & CGR3 & GZMB & HCST & \begin{tabular}{|l|} 
HPRT1 \\
\end{tabular} & \begin{tabular}{|l|l|} 
IL17RA \\
\end{tabular} & IL2RA & KLRG1 & \begin{tabular}{|l|} 
LAMP1 \\
\end{tabular} & NKG2C & \begin{tabular}{|l|} 
NKG2D \\
\end{tabular} & \begin{tabular}{|l|l} 
NKP30 \\
\end{tabular} & NKp80 & \begin{tabular}{|l|l|} 
NKP46 \\
\end{tabular} & NKR-P1 & PRF1 & IYROBP \\
\hline mm1 (2) & 2,73 & 7,46 & 4,41 & 6,87 & 2,77 & 14,22 & 12,04 & $-1,21$ & 1,75 & \begin{tabular}{|l|}
40,22 \\
\end{tabular} & 3,53 & 10,78 & $\overline{8,40}$ & 22,01 & 5,28 & 1,39 & \begin{tabular}{|l|}
11,08 \\
\end{tabular} & \begin{tabular}{|l|}
$-33,59$ \\
\end{tabular} & 3,53 & \begin{tabular}{|l|}
140,07 \\
\end{tabular} & 1,27 \\
\hline mm1 (3) & 4,23 & 4,72 & 8,46 & 4,14 & 3,81 & 13,27 & 4,89 & 0,00 & 2,13 & 25,63 & 3,56 & 8,57 & 7,26 & 16,45 & 3,27 & 1,74 & 8,51 & $-14,93$ & 4,41 & 66,26 & 2,19 \\
\hline mm3 (2) & 5,39 & 22,78 & 2,16 & 7,21 & 4,79 & 18,77 & 36,00 & 2,57 & 2,03 & 9,25 & 2,27 & 14,72 & 5,28 & 20,53 & 9,65 & 6,96 & 15,14 & 1,04 & 5,13 & 85,04 & 2,91 \\
\hline mm3 (3) & 5,10 & 28,44 & 1,77 & 10,70 & 9,13 & 18,38 & 22,63 & 3,51 & 3,41 & $-2,13$ & 1,89 & 15,14 & 1,87 & 10,93 & 17,03 & 5,35 & 23,59 & 1,93 & 3,16 & 20,53 & 4,41 \\
\hline mm5 (2) & 6,11 & 4,38 & 1,87 & 4,76 & 2,30 & 6,23 & 17,03 & 1,15 & 1,46 & 7,16 & 1,39 & 5,70 & 3,18 & 6,54 & 3,68 & 1,92 & 12,47 & $-1,78$ & 2,79 & 34,06 & 1,16 \\
\hline \begin{tabular}{|l|}
$\mathrm{mm6}(2)$ \\
\end{tabular} & 8,34 & 12,64 & 4,92 & 1,91 & 8,69 & 10,34 & 28,25 & 1,55 & 2,08 & 2,45 & 1,47 & 13,93 & 6,19 & 41,07 & 14,42 & 4,03 & \begin{tabular}{|l|}
18,51 \\
\end{tabular} & $-25,11$ & 7,21 & 32,67 & 8,57 \\
\hline $\operatorname{mm6}(3)$ & 7,62 & 13,45 & 4,89 & 1,27 & 5,39 & 14,32 & 25,63 & 1,47 & 1,79 & $-2,87$ & 1,17 & 8,94 & 2,97 & 42,22 & 10,70 & 3,07 & 11,39 & $-34,54$ & 4,96 & 2,41 & 5,21 \\
\hline mm7 (2) & 1,41 & 6,50 & $-1,11$ & 1,37 & 1,96 & 8,17 & 7,67 & 1,01 & 0,00 & 11,55 & $-1,05$ & 4,76 & 3,03 & 13,27 & 4,20 & 1,71 & 10,56 & $-1,87$ & 1,80 & $\begin{array}{ll}47,84 \\
\end{array}$ & 1,09 \\
\hline mm7 (3) & $-5,94$ & 1,37 & $-4,86$ & $-3,10$ & 3,07 & 7,41 & 2,04 & $-1,80$ & $-1,15$ & 3,68 & $-7,78$ & $-1,16$ & 1,95 & 1,68 & 1,09 & $-1,13$ & 1,33 & $-1,96$ & $-1,97$ & 5,39 & 1,72 \\
\hline mm8 (2) & 2,83 & 5,10 & 1,85 & $-1,34$ & \begin{tabular}{|l|l|}
2,77 \\
\end{tabular} & 4,23 & \begin{tabular}{|l|}
17,39 \\
\end{tabular} & 1,04 & 1,07 & 2,69 & $\begin{array}{ll}1,26 \\
\end{array}$ & 3,34 & 5,43 & 9,45 & 3,81 & 2,50 & \begin{tabular}{|l|}
11,39 \\
\end{tabular} & $-25,99$ & 3,63 & \begin{tabular}{|l|}
46,21 \\
\end{tabular} & 2,46 \\
\hline mm9 (2) & 7,36 & 18,25 & 3,23 & $-11,39$ & 10,13 & 11,47 & 13,27 & 2,83 & 4,20 & $-7,06$ & 1,72 & 12,04 & 4,11 & 32,45 & 23,92 & 3,86 & \begin{tabular}{|l|}
17,51 \\
\end{tabular} & $-10,70$ & 6,15 & 12,13 & 5,62 \\
\hline mm10 (2) & 2,95 & 10,56 & 2,20 & 4,56 & 3,41 & 4,92 & 32,45 & 1,57 & 1,65 & 18,25 & 2,31 & 8,75 & 4,20 & 13,93 & 6,23 & 4,53 & 18,51 & $-1,51$ & 8,40 & \begin{tabular}{|l|}
87,43 \\
\end{tabular} & 3,34 \\
\hline mm10 (3) & 6,19 & 11,71 & 2,31 & 11,08 & 7,26 & 7,57 & 42,52 & 1,66 & 2,11 & 18,90 & 3,97 & 10,78 & 5,10 & 15,14 & 9,78 & 4,59 & 12,04 & $-12,55$ & 9,65 & 83,29 & 6,32 \\
\hline mm17 (2) & 1,89 & 9,85 & 2,10 & 22,47 & 9,45 & 12,91 & 6,19 & 2,11 & 1,01 & $-6,68$ & 1,13 & 3,84 & 2,07 & 8,17 & 2,43 & 2,48 & 11,00 & 2,00 & 2,36 & 2,10 & 2,99 \\
\hline mm18 (2) & 8,63 & 21,86 & 5,24 & 5,43 & 8,00 & 10,13 & 15,56 & 2,38 & 2,73 & $-2,35$ & $-1,23$ & 13,64 & 3,78 & 76,64 & 9,78 & 5,94 & 22,47 & $-2,64$ & 7,57 & 16,68 & 3,16 \\
\hline mm18 (3) & 6,92 & 13,64 & 4,86 & 3,86 & 14,12 & 23,75 & 7,11 & 1,78 & 2,00 & 1,82 & 1,17 & 9,13 & 6,50 & 48,50 & 6,54 & 2,30 & 11,39 & $-5,31$ & 3,27 & 20,97 & 4,63 \\
\hline Imm19(2) & 2,20 & 18,13 & 1,27 & 1,20 & 9,78 & 10,20 & 1,13 & 4,35 & 6,41 & $-8,63$ & 3,05 & 2,91 & 1,06 & 7,41 & 6,50 & 5,74 & 11,31 & 1,37 & $-5,62$ & \begin{tabular}{|l|}
$-10,48$ \\
\end{tabular} & 4,44 \\
\hline mm19 (3) & $-1,16$ & 2,33 & $-3,18$ & $-5,50$ & 5,66 & 2,35 & $-6,45$ & 3,07 & 2,93 & $-1,22$ & 0,00 & 1,04 & 1,60 & $-1,03$ & 1,80 & 1,61 & 1,60 & 3,53 & $-14,52$ & $-1,91$ & 2,08 \\
\hline mm22 (3) & $-3,43$ & $-4,11$ & $-4,63$ & $-1,71$ & $-3,12$ & $-1,40$ & \begin{tabular}{|c|}
$-3,81$ \\
\end{tabular} & $-1,02$ & $-1,19$ & 1,19 & $-4,69$ & $-5,10$ & $-1,10$ & $-4,76$ & $-3,86$ & $-1,23$ & $-4,47$ & 3,20 & $-9,45$ & $-1,56$ & $-3,23$ \\
\hline Imm25 (2) & 4,03 & 4,76 & 2,06 & $-1,65$ & 1,73 & 5,98 & 3,89 & 1,43 & 1,38 & 7,5 & $-1,08$ & 3,7 & 4,06 & 5,46 & 4,89 & 2,36 & 11,00 & $-1,53$ & 2,13 & $\begin{array}{l}79,89 \\
\end{array}$ & 1,23 \\
\hline mm25 (3) & $-1,31$ & 2,73 & $-3,01$ & $-12,47$ & 1,11 & 2,14 & $-1,28$ & 1,55 & $-1,54$ & $-29,24$ & $-2,60$ & 1,17 & $-2,69$ & 1,57 & 2,01 & 1,01 & 4,72 & 3,43 & $-2,14$ & $-1,40$ & $-2,01$ \\
\hline \begin{tabular}{|l}
$\mathrm{mm26}(2)$ \\
\end{tabular} & 4,56 & 17,03 & 3,43 & $-1,25$ & 5,90 & 17,03 & 24,08 & 1,21 & 1,96 & \begin{tabular}{ll|}
14,22 \\
\end{tabular} & 1,43 & 10,70 & \begin{tabular}{|l|}
13,09 \\
\end{tabular} & 25,11 & 14,32 & 4,41 & 18,25 & \begin{tabular}{|c|}
$-5,66$ \\
\end{tabular} & 7,62 & 115,36 & 5,24 \\
\hline mm26 (3) & 4,66 & 22,16 & 3,56 & $-1,93$ & 9,38 & 23,59 & 27,67 & $-1,11$ & 3,39 & 6,50 & 1,15 & 19,56 & 10,13 & 46,21 & 17,27 & 3,16 & 24,08 & $-9,92$ & 5,46 & 53,45 & 6,96 \\
\hline mm27 (2) & $-1,71$ & 2,20 & $-2,11$ & $-2,28$ & 3,18 & 6,1 & 1,99 & 1,08 & 1,22 & 3,6 & 1,0 & 2, & 2,7 & 2,51 & 3,89 & 2,36 & 1,96 & $-1,54$ & 2,51 & 2,55 & 3,84 \\
\hline mm27 (3) & 1,53 & 6,59 & $-1,15$ & 1,38 & 3,48 & 12,73 & 5,58 & $-1,17$ & 1,67 & 14,72 & 1,32 & 10,27 & 2,69 & 7,01 & 5,98 & 2,97 & 6,15 & $-7,73$ & 3,56 & 25,46 & 3,94 \\
\hline Imm29(3) & $-1,83$ & $-1,79$ & $-2,14$ & $-3,46$ & $-1,84$ & 1,65 & 1,21 & $-1,57$ & $-1,84$ & 1,74 & $-2,53$ & $-4,96$ & 1,22 & $-2,39$ & $-1,72$ & $-1,85$ & $-1,75$ & $-18,25$ & $-4,35$ & 1,73 & $-2,07$ \\
\hline Imm33 (2) & 3,94 & 17,75 & 2,60 & 1,46 & 6,36 & 10,56 & 3,63 & 5,03 & 4,50 & $-4,79$ & 3,36 & 12,38 & 1,5 & 15,56 & 11,08 & 5,31 & \begin{tabular}{|l|}
18,90 \\
\end{tabular} & $-1,61$ & 4,53 & 2,08 & 4,53 \\
\hline mm33 (3) & 6,92 & 14,32 & 2,95 & 1,58 & 4,08 & 11,79 & 3,94 & 5,06 & 2,87 & 1,09 & 2,75 & 13,55 & 2,43 & 9,71 & 10,34 & 4,79 & 13,74 & $-3,03$ & 3,14 & 7,31 & 2,57 \\
\hline Imm39 (2) & 2,60 & 2,07 & 1,19 & $-2,07$ & 4,26 & 2,55 & 1,66 & $-1,01$ & 1,60 & 10,7 & 2,1 & 2,38 & 3,29 & 4,5 & 2,13 & 1,06 & 2,23 & \begin{tabular}{|l|}
$-95,01$ \\
\end{tabular} & 1,61 & 2,43 & 3,36 \\
\hline mm39 (3) & $-1,40$ & $-1,38$ & $-5,94$ & $-6,41$ & 5,62 & 3,81 & $-1,78$ & $-1,49$ & $-1,37$ & 3,41 & $-1,59$ & $-1,02$ & 2,43 & $-1,43$ & $-1,16$ & $-1,01$ & $-1,45$ & $-116,16$ & $-2,36$ & 1,06 & 3,25 \\
\hline Imm40(2) & 1,75 & 4,00 & 1,27 & $\begin{array}{l}-8,69 \\
\end{array}$ & \begin{tabular}{ll|}
4,23 \\
\end{tabular} & 18,13 & 2,66 & 1,01 & 1,54 & 25,46 & 3,23 & 3,20 & 7,73 & 4,53 & 3,03 & 1,32 & 4,50 & $-46,85$ & $-1,29$ & 13,00 & 2,99 \\
\hline mm40 (3) & 2,27 & 3,51 & 1,21 & $-5,28$ & 4,00 & 15,03 & 2,27 & 1,48 & 1,77 & 9,32 & 1,07 & 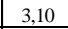 & 6,2 & 3,97 & 2,71 & 2,58 & 4,99 & $-34,30$ & 1,47 & 6,92 & 2,23 \\
\hline mm45 (2) & 3,23 & 3,29 & 1,60 & $-3,92$ & 2,8 & 11,4 & 1,95 & $-1,48$ & 1,35 & 20,6 & 3,2 & 4,0 & 8,5 & 3,46 & 1,57 & 1,13 & 3,05 & $-89,26$ & 1,21 & 26,91 & 2,43 \\
\hline mm45 (3) & 4,11 & 5,06 & 3,48 & 1,16 & 2,68 & 32,90 & 3,10 & 1,06 & 1,42 & 38,59 & 2,3 & 6,6 & 12,38 & 4,38 & 2,36 & 1,99 & 6,02 & $-64,89$ & 1,59 & 43,11 & 2,04 \\
\hline Imm47 (2) & 8,51 & 5,86 & 4,72 & $-3,48$ & 2,89 & 26,91 & 3,63 & 1,09 & 1,57 & 95,67 & 2,17 & 3,68 & 14,52 & 9,00 & 2,73 & 1,91 & 11,16 & $-44,32$ & 1,59 & $\begin{array}{l}49,52 \\
\end{array}$ & 3,53 \\
\hline mm47 (3) & 4,23 & 4,79 & 1,82 & $-1,22$ & 3,68 & 30,27 & 4,38 & $-1,39$ & 1,40 & 133,44 & $-1,62$ & 3.6 & 16,22 & 8,63 & 2,36 & 1,69 & 9,71 & $-54,95$ & 1,46 & 98,36 & 3,14 \\
\hline \begin{tabular}{|l}
$\mathrm{mm49}(2)$ \\
\end{tabular} & 1,80 & 1,24 & $-1,39$ & $-4,50$ & 1,60 & 1,92 & $-1,67$ & 1,22 & 1,04 & 6,11 & $-1,12$ & 1,05 & 2,5 & 1,78 & 1,51 & 1,36 & 1,02 & 1,04 & $-1,45$ & 3,86 & 1,44 \\
\hline mm49 (3) & 5,62 & 2,57 & 1,54 & $-1,82$ & 2,14 & 8,22 & $-1,28$ & 1,09 & 1,91 & 30,48 & 1,39 & 3,73 & 7,73 & 3,86 & 2,50 & 1,75 & 2,48 & $-4,99$ & $-1,56$ & 11,16 & 2,08 \\
\hline \begin{tabular}{|l|l} 
mm52 (2) \\
\end{tabular} & 2,39 & 1,89 & 2,23 & 1,20 & 2,77 & 11,31 & 2,57 & $-1,41$ & 1,39 & 21,56 & 1,89 & 3,20 & 9,51 & 7,41 & 1,47 & 1,39 & 5,13 & $-4,59$ & $-1,06$ & \begin{tabular}{ll|}
67,18 \\
\end{tabular} & 2,28 \\
\hline mm52 (3) & 1,85 & $-1,53$ & 1,60 & 0,00 & 1,19 & 3,78 & $-1,25$ & $-1,57$ & 1,08 & 15,78 & 1,34 & $-1,24$ & 7,67 & 3,78 & $-1,62$ & $-1,05$ & $-1,12$ & $-2,91$ & $-1,33$ & 41,07 & 1,20 \\
\hline LVL & CD8B & CD94 & CD96 & FASLG & FCER1G & FCGR3 & GZMB & HCST & HPRT1 & IL17RA & IL2RA & KLRG1 & LAMP1 & NKG2C & \begin{tabular}{|l|} 
NKG2D \\
\end{tabular} & \begin{tabular}{|l|} 
NKPP30 \\
\end{tabular} & NKp80 & NKP46 & NKR-P1 & PRF1 & YROBP \\
\hline \begin{tabular}{|l|}
$\mathrm{mm2}(2)$ \\
\end{tabular} & 1,75 & 2,85 & 2,36 & 4,44 & 2,28 & 16,68 & 5,06 & $-1,69$ & 1,17 & 14,83 & 2,85 & 2,93 & 4,92 & 3,53 & 2,23 & $-1,04$ & 3,53 & $-11,31$ & $-1,32$ & 18,13 & $-1,04$ \\
\hline $\mathrm{mm} 2(3)$ & 2,07 & 3,07 & 3,46 & 3,89 & 2,13 & 16,45 & 3,25 & 1,12 & 1,13 & 11,88 & 2,81 & 4,1 & 3,48 & 2,57 & 2,06 & 1,29 & 3,92 & 1,26 & 1,25 & 10,20 & $-1,01$ \\
\hline \begin{tabular}{|l|}
$\mathrm{mm4}(2)$ \\
\end{tabular} & 3,97 & 13,55 & 2,25 & 1,15 & 6, & 2,6 & 26,54 & 2,46 & 2,83 & $-2,60$ & 1,5 & 9, & 2,36 & 13,74 & 6,50 & 5,24 & \begin{tabular}{|l|}
23,75 \\
\end{tabular} & $-42,52$ & 11,79 & 11,16 & 7,57 \\
\hline $\operatorname{mm4}(3)$ & 3,81 & 9,45 & 2,27 & $-1,10$ & 6,5 & 6,9 & 18,64 & 2,30 & 3,01 & 2,71 & 1,78 & 12,04 & 3,94 & 7,36 & 6,68 & 5,70 & 13,83 & $-56,49$ & 8,28 & 33,59 & 7,01 \\
\hline mm11 (2) & 1,30 & $-1,58$ & 1,38 & 1,44 & $-1,20$ & $-1,59$ & $-1,78$ & $-1,45$ & 1,04 & 2,95 & 1,57 & 1,12 & 1,96 & $-1,68$ & $-1,35$ & $-1,85$ & $-1,75$ & $-1,21$ & $-1,49$ & 4,08 & $-1,28$ \\
\hline mm11 (3) & 1,79 & $-1,55$ & 2,07 & $-1,17$ & $-3,76$ & $-3,66$ & $-6,63$ & $-1,58$ & $-1,14$ & 10,70 & 1,35 & $-2,27$ & 2,87 & $-4,44$ & $-2,35$ & $-2,91$ & $-2,83$ & $-1,61$ & $-15,35$ & 9,06 & $-3,10$ \\
\hline mm12 (2) & 1,97 & 4,08 & 1,18 & \begin{tabular}{|l|}
$-13,55$ \\
\end{tabular} & 4,69 & 8,1 & 1,89 & \begin{tabular}{|l|}
$-1,93$ \\
\end{tabular} & 1,58 & 2,22 & $-1,3$ & 3, & 3, & 2,8 & 2,10 & $-1,36$ & 3 & $-6,06$ & $-2,33$ & 20 & 2,55 \\
\hline mm12 (3) & 5,39 & 7,01 & 2,41 & $-29,65$ & 8,6 & & 4,11 & 1,85 & 3,56 & $-1,0$ & 3, & 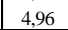 & 3,34 & 5,35 & 5,17 & 2,75 & .51 & $-61,82$ & 2,41 & 01 & 6,77 \\
\hline Imm20(2) & \begin{tabular}{|l|}
$-2,25$ \\
\end{tabular} & \begin{tabular}{|l|}
$-6,63$ \\
\end{tabular} & $\begin{array}{l}-2,46 \\
\end{array}$ & \begin{tabular}{|l|}
$-3,61$ \\
\end{tabular} & \begin{tabular}{|l|l|}
$-1,01$ \\
\end{tabular} & $-2,27$ & $\begin{array}{l}-7,57 \\
\end{array}$ & \begin{tabular}{|c|}
$-3,63$ \\
\end{tabular} & \begin{tabular}{|c|}
$-2,71$ \\
\end{tabular} & 4,17 & $-2,62$ & $\begin{array}{l}-5,06 \\
\end{array}$ & 1,74 & \begin{tabular}{|l|}
$-5,31$ \\
\end{tabular} & $-4,79$ & $-6,28$ & \begin{tabular}{|l|l|}
$-7,89$ \\
\end{tabular} & 1,13 & $-4,79$ & 1,24 & $-1,29$ \\
\hline mm20 (3) & $-1,84$ & $-2,10$ & $-1,45$ & $-1,55$ & $-1,96$ & 1,27 & $-1,87$ & $-1,58$ & $-1,85$ & $-4,44$ & $-4,86$ & $-1,47$ & $-1,60$ & $-2,07$ & $-2,31$ & $-1,75$ & $-1,27$ & 11,39 & $-2,19$ & $-1,78$ & $-2,28$ \\
\hline mm28 (2) & 2,30 & 9,85 & 2,97 & 2,1 & 5 , & 13,4 & 8,2 & 1,02 & 2,22 & 4,0 & 2,6 & 9,1 & 4,9 & 15,35 & 4,38 & 2,51 & 15,45 & $-50,21$ & 2,31 & 12,47 & 3,36 \\
\hline mm28 (3) & 3,29 & 6,59 & 2,79 & & 2, & 1,6 & 6,7 & 1,89 & 2,36 & $-1,3$ & 3, & & 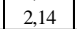 & 7,94 & 3,92 & 3,39 & 8,40 & $-53,08$ & 4,82 & 4 & 2,45 \\
\hline Imm30(2) & 3,81 & 5,74 & 2,57 & $-2,0$ & 3,0 & 8,2 & 8,7 & 2,85 & 1,73 & 3,7 & 1,8 & 3, & 3,5 & 6 & 2,85 & 4,26 & 16,22 & $-4,20$ & 5,13 & 28,64 & 2,08 \\
\hline mm30 (3) & 3,71 & 11,96 & 2,71 & $-2,55$ & 5,28 & 13,74 & 5,94 & 3,63 & 2,30 & 3,68 & 2,19 & 7,06 & 3,25 & 8,88 & 6,06 & 3,81 & 13,64 & $-8,57$ & 4,66 & 15,45 & 3,34 \\
\hline mm32 (2) & 8,46 & 16,56 & 3,58 & 2,23 & 3,12 & 6,06 & 1,36 & 1,95 & 2,62 & 10,06 & 3,3 & 6,96 & 4,38 & 23,10 & 11,24 & 4,66 & 18,77 & $-21,56$ & 1,78 & 62,68 & 2,93 \\
\hline mm32 (3) & 10,63 & 5,58 & 3,36 & 1,78 & 2,85 & 5,4 & 2,20 & 1,61 & 2,71 & & 5,5 & 7,1 & 4,9 & 6,87 & 5,58 & 3,41 & 5,39 & $-62,25$ & 2,30 & 9,25 & 2,85 \\
\hline mm35 (2) & $-1,06$ & 1,04 & $-1,05$ & $-1,42$ & 1,74 & $-1,27$ & $-1,12$ & $-1,12$ & 1,41 & $-32,22$ & $-1,4$ & 1,3 & $-1,6$ & 1,2 & 1,35 & $-1,33$ & $-1,55$ & $-4,20$ & 1,45 & $-10,06$ & 1,56 \\
\hline mm35 (3) & 1,54 & 1,37 & 1,72 & 2,48 & 1,48 & 1,04 & 3,66 & 1,58 & 1,61 & $-3,14$ & 1,02 & 1,21 & $-1,19$ & 2,03 & 2,27 & 1,74 & 1,39 & $-27,47$ & 4,00 & $-1,52$ & 1,89 \\
\hline mm36 (2) & 5,90 & 19,84 & 2,93 & $-3,10$ & 2,13 & 9,85 & 6,32 & 2,01 & 1,69 & 2,81 & 2,73 & 29,04 & 6,06 & 14,03 & 10,48 & 4,06 & 21,41 & $-39,95$ & 3,29 & 55,33 & 2,36 \\
\hline mm36 (3) & 6,68 & 8,22 & 3,27 & $-9,65$ & 3,58 & 9,58 & 3,01 & 1,87 & 1,82 & 11,00 & 3,34 & 8,06 & 8,57 & 5,21 & 7,26 & 2,85 & 5,74 & $-33,59$ & 2,19 & 31,12 & 3,71 \\
\hline mm37 (3) & 4,63 & 1,53 & 3,16 & $-1,66$ & $-1,74$ & 1,41 & $-1,64$ & $-1,23$ & 2,20 & 2,55 & 2,9 & 2,04 & 1,96 & 1,55 & 1,51 & 1,02 & 2,64 & $-27,10$ & $-1,20$ & 2,71 & $-1,37$ \\
\hline \begin{tabular}{|l|l}
$\mathrm{mm} 38(2)$ \\
\end{tabular} & 3,78 & 8,34 & 2,95 & $-1,72$ & 3,5 & & 2,2 & 1,83 & 1,93 & $-2,6$ & & 14, & & & 5,03 & 2,30 & 9,99 & $-20,11$ & 1,47 & 2,89 & 2,97 \\
\hline mm38 (3) & 3,73 & 6,82 & 1,43 & 1,10 & 6,68 & 41,36 & 3,97 & 2,43 & 2,01 & 13,36 & 4,82 & 10,13 & 7,06 & 1,85 & 5,31 & 3,29 & 5,98 & $-2,23$ & 5,90 & 20,11 & 5,17 \\
\hline Imm41 (2) & \begin{tabular}{|l|}
18,00 \\
\end{tabular} & 6,19 & 4,50 & $\begin{array}{l}-6,82 \\
\end{array}$ & 2,22 & 64,89 & 4,00 & 1,41 & 3,53 & 22,32 & 6,54 & 6,73 & 11,63 & 6,19 & 4,44 & 3,46 & 7,67 & $-109,90$ & 2,60 & \begin{tabular}{|l|}
43,71 \\
\end{tabular} & 2,20 \\
\hline mm41 (3) & 9,92 & 8,57 & 2,03 & $-4,23$ & 2,43 & 49,87 & 6,19 & 1,91 & 2,08 & 17,63 & 5,0 & 9,1 & 10,34 & 8,69 & 6,06 & 5,43 & 18,25 & $-83,87$ & 2,91 & 81,57 & 2,20 \\
\hline mm44 (2) & 6,32 & 8,63 & 2,35 & $-1,2$ & 2,30 & 14, & 4,3 & 1,39 & 2,2 & 64,4 & 4,2 & 7,6 & 9,58 & 5 , & 6,11 & 2,16 & 5,70 & $-54,57$ & 1,61 & \begin{tabular}{|l|l|}
79,89 \\
\end{tabular} & 1,53 \\
\hline mm44 (3) & 4,89 & 7,84 & 2,25 & 1,4 & 3,10 & 13, & 3,81 & 1,09 & 1,87 & 49,52 & 2,95 & 6,82 & 9,25 & 4,79 & 4,53 & 1,62 & 5,62 & $-17,39$ & 2,04 & 45,89 & 2,28 \\
\hline Imm46 (2) & 6,68 & 2,58 & 2,87 & 1,57 & $-1,17$ & 4,23 & 1,88 & 1,19 & 1,67 & 4,03 & $-1,68$ & 2,83 & 3,81 & 3,25 & 1,68 & 1,39 & 2,33 & $-6,02$ & 1,03 & 7,06 & 1,02 \\
\hline mm46 (3) & 4,26 & 1,91 & 2,16 & $-1,05$ & 2,28 & 12,38 & 1,23 & 0,00 & 1,06 & 7,06 & $-2,16$ & 2,28 & 5,70 & 2,30 & 1,31 & $-1,02$ & 2,58 & $-1,43$ & $-1,91$ & 12,04 & 1,65 \\
\hline n48 (2) & 1,89 & 1,34 & $-1,10$ & $-4,92$ & 1,38 & 3,76 & $-1,52$ & $-1,0$ & 1,15 & 15,24 & $-1,8$ & 1,6 & 5,6 & 1,10 & 1,54 & 1,04 & 1,49 & $-1,35$ & $-1,61$ & 11,08 & 1,77 \\
\hline mm48 (3) & 1,75 & $-1,20$ & 1,21 & $-6,87$ & 1, & 2, & $-1,96$ & $-1,35$ & 1,33 & 13,00 & $-1,4$ & $-1,09$ & 7,41 & 1,14 & $-1,12$ & $-1,22$ & $-1,59$ & $-6,50$ & $-1,67$ & 7,52 & 1,45 \\
\hline mm50 (3) & 9,78 & 5,43 & 2,77 & 1,42 & 1,71 & 14,42 & 6,59 & 1,38 & 1,71 & \begin{tabular}{|l|l|}
72,50 \\
\end{tabular} & 7,11 & 15,14 & 11,24 & 7,89 & 5,17 & 3,81 & 8,88 & $-2,66$ & 8,34 & 124,50 & $-1,13$ \\
\hline
\end{tabular}




\section{Acknowledgement}

First of all I would like to thank Professor Dr. Lutz Walter for supervising my doctoral thesis, for his support, for scientific discussions and for the possibility to attend several international conferences.

I also want to thank my thesis committee members, Professor Dr. Holger Reichardt and Professor Dr. Michael Kessel for supporting me during my research at the DPZ. In addition, I would like to thank Professor Dr. Holger Reichardt for being the co-reviewer of my thesis.

Special thanks are addressed to Professor Dr. Aftab Ansari for the cooperation. Thanks for providing the samples of SIV infected rhesus macaques, to answer all my questions and for providing me all information I wanted to know.

I also want to thank Dr. Markus Brameier for his bioinformatics support and to make it possible to handle the mass of sequencing data.

A special thank is addressed to Meike Hermes for her big support during the doctoral time. I would like to thank her for all her useful comments on the manuscript, for the scientific and non-scientific discussions, all the conference and other trips we did together, and that I have never lost my way!

Thanks to Astrid Backhaus and Nico Westphal for their help in the lab, and Astrid for the funny time during lunchtime.

I would like to thank all former and current members of the Primate Genetics lab for their general support and a great working atmosphere.

I would like to thank Stefan Bruns for his support with the manuscript and for pushing me to never give up.

Last but not least I would like to thank all my friends and my family for their never ending support 